\title{
MATHEMATICAL RECREATIONS
}

\section{AND ESSAYS}

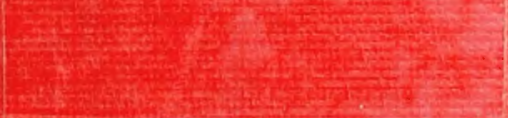


$Q A$
95
$B 18$
1922

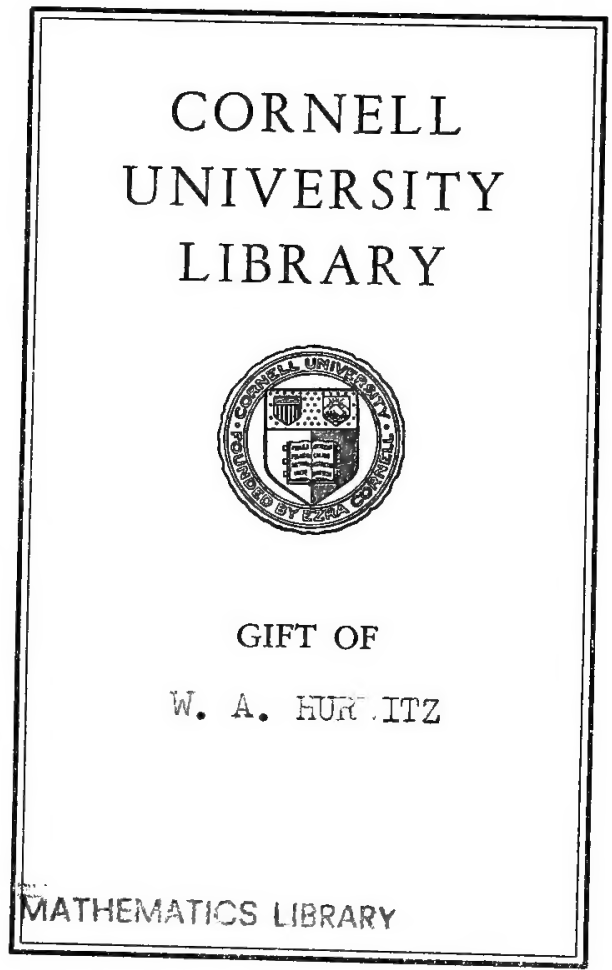


OA $95 . B 181922$

Mathematical recreations and essays,

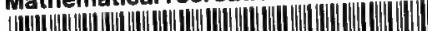

.

31924001539562 math

\section{Date Due}

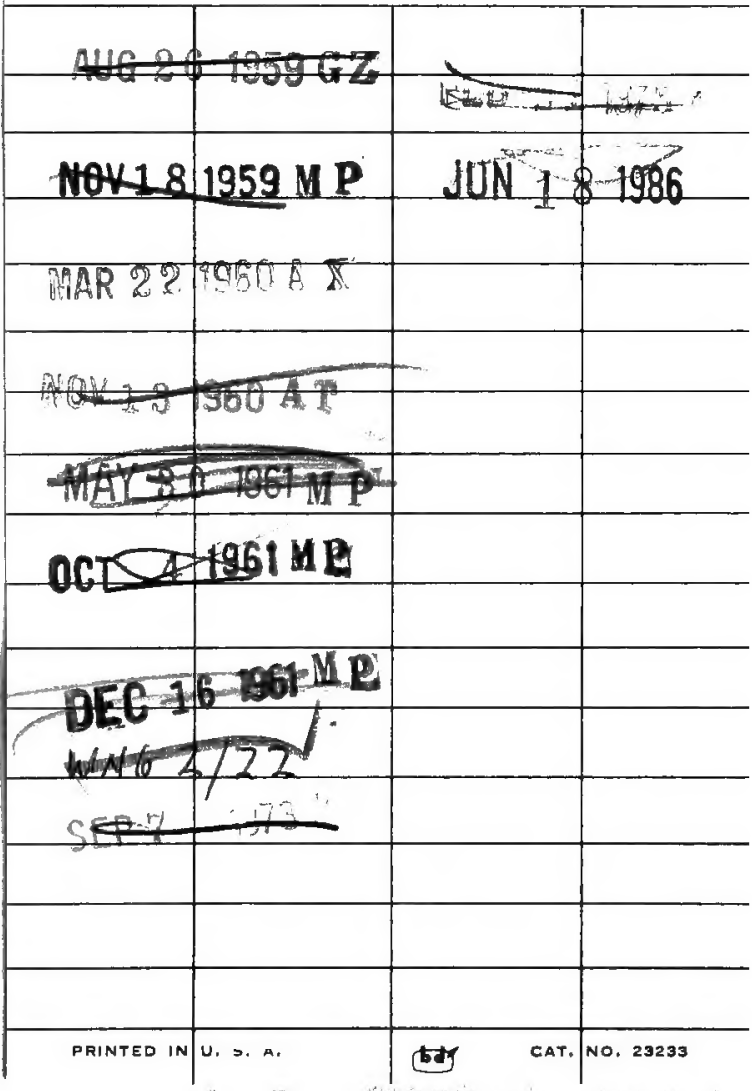




\section{Cornell University Library}

The original of this book is in the Cornell University Library.

There are no known copyright restrictions in the United States on the use of the text. 


MATHEMATICAL RECREATIONS AND ESSAYS 


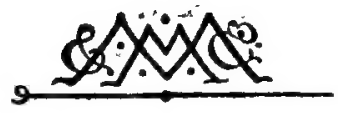

\author{
MACMILLAN AND CO., Limited \\ LONDON - BOMBAY - CALCUTTA - MADRAS \\ MELBOURNE
}
THE MACMILLAN COMPANY
NEW YORK - BOSTON - CHICAGO
DALLAS + SAN FRANCISCO

'THE MACMILLAN CO. OF CANADA, LtD.

TORONTO 


\section{MATHEMATICAL \\ RECREATIONS AND ESSAYS}

$\mathbf{B T}$

W. W. ROUSE BALL,

EELLOW OF TRINITY COLLEGR, CAMBRIDGE.

TENTH EDITION.

MACMILLAN AND CO., LIMITED ST MARTIN'S STREET, LONDON 1922

[All right reserved]

7 b 


\section{Coptright}

First Edition, 1892.

Second Edition, 1892.

Third Edition, 1896.

Fourth Edition, 1905.

Fifth Edition, 1911.

Sixth Edition, 1914.

Seventh Edition, 1917.

Eighth Edition, 1919.

Ninth Edition, 1920.

Tenth Edition, 1922.

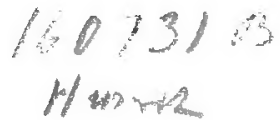

Tranite. fiom olite sia

rpl for c. C.

PRINTED IN GREAT BRITAIN 


\section{PREFACE.}

THIS book contains descriptions of various problems of the kind on some analogous questions. I have excluded all matter which involves advanced mathematics. I hasten to add that the conclusions are of no nractical nse and that most of the results are

\section{FRAGILE PAPER}

Please handle this book with care, as the paper is fragile. A decision on replacement is pend1ng. roceeds further he is at least link I may say that many of ing, not a few are associated thematicians, while hitherto : not been easily accessible to $r$ matter has been added since $t$ insertions made since 1911, ave had to be placed where

room Ior tnem coula pest pe rouna.

In the first nine editions the book was divided into two parts, most of the second being devoted to questions of a historical character. There is ample precedent for including such matter in books of mathematical recreations, but I have long felt that much of the interesting and out-of-the-way information there brought together would find a better place elsewhere. Accordingly in this edition I have given up the division into two parts, and have struck out most of the chapters in the second part such as those on calculating machines, parallels, hyperspace, atoms and ether, time and time measurers, etc.: earlier editions are accessible for the matter now omitted.

As now presented, the book contains only sixteen chapters, of which the subjects are shown in the Table of Contents. Several 
Coptrigl

First Edition,

Second Edition,

Third Edition,

Fourth Edition,

Fifth Edition,

Sixth Edition,

Seventh Edition

Eighth Edition,

Ninth Edition, 1920.

Tenth Edition, 1922.

$160731: 3$

Hm,

Trouser, from olin stake

ret R or 0.1

PRINTED IN GREAT BRITAIN 


\section{PREFACE.}

THIS book contains descriptions of various problems of the kind

1 usually termed Mathematical Recreations, and a few Essays on some analogous questions. I have excluded all matter which involves advanced mathematics. I hasten to add that the conclusions are of no practical use, and that most of the results are not new. If therefore the reader proceeds further he is at least forewarned. At the same time I think I may say that many of the questions discussed are interesting, not a few are associated with the names of distinguished mathematicians, while hitherto several of the memoirs quoted have not been easily accessible to English readers. A great deal of new matter has been added since the work was first issued in 1892, but insertions made since 1911, when the book was stereotyped, have had to be placed where room for them could best be found.

In the first nine editions the book was divided into two parts, most of the second being devoted to questions of a historical character. There is ample precedent for including such matter in books of mathematical recreations, but I have long felt that much of the interesting and out-of-the-way information there brought together would find a better place elsewhere. Accordingly in this edition I have given up the division into two parts, and have struck out most of the chapters in the second part such as those on calculating machines, parallels, hyperspace, atoms and ether, time and time measurers, etc.: earlier editions are accessible for the matter now omitted.

As now presented, the book contains only sixteen chapters, of which the subjects are shown in the Table of Contents. Several 
of the questions mentioned in the first five chapters are of a somewhat trivial character, and had they been treated in any standard English work to which I could have referred the reader, I should have left them out: in the absence of such a work, I thought it better to insert them and trust to the judicious reader to omit them altogether or to skim them as he feels inclined. The twelfth chapter is new. I may add that in discussing problems where the complete solutions are long or intricate $I$ have been generally content to indicate memoirs or books in which the methods are set out at length, and to give a few illustrative examples. In some cases I have also stated problems which still await solution.

I have inserted detailed references, as far as I know them, to the sources of the various questions and solutions given; also, wherever I have given only the result of a theorem, I have tried to indicate authorities where a proof may be found. In general, unless it is stated otherwise, I have taken the references direct from the original works; but, in spite of considerable time spent in verifying them, I dare not suppose that they are free from all errors or misprints. I shall be grateful for notices of additions or corrections which may occur to any of my readers.

\section{W. W. ROUSE BALL.}

Trinity College, Cambridge.

April, 1922. 


\section{TABLE OF CONTENTS.}

Chapter I. Arithmetical Recreations.

Bachet. Ozanam. Montucla . . . . . . . . . 2

Elementary Questions on Numbers . . . . . . . . 3

Determination of a number selected by someone. . . . 4

Prediction of the result of certain operations . . . 7

Problems involving two numbers o. . . . . . 10

Miscellaneous Problems . . . . . . . . 12

Problems with a Series of Numbered Things . . . . . . 14

Medieval Problems . . . . . . . . . . . 18

The Josephus Problem. Decimation . . . . . . . 23

Addendum on Solutions . . . . . . . . . . 27

Chapter II. Arithmetidal Recreations (Continued).

Arithmetical Fallacies . . . . . . . . . . 28

Paradoxical Problems . . . . . . . . . . 31

Permutation Problems . . . . . . . . . . 32

Bachet's Weights Problem . . . . . . . . . 34

Problems in Higher Arithmetio . . . . . . . . . B6

Primes. Mersenne's Numbers. Perfect Numbers . • $\quad 37$

Unsolved Theorems by Euler, Goldbach, Lagrange . . $\quad 39$

Fermat's Theorem on Binary Powers . . . . . . 39

Fermat's Last Theorem . . . . . . . . . 40 
Chapter III. Geonetrical Recreationg.

Geometrical Fallacies.

Paradoxical Problems

Map-Colouring Theorem

Physioal Configuration of a Country

Addendum on a Solution.

Chapter IV. Geometrical Recreations (Continued).

Statical Games of Position

Three-in-a-row. Extension to $p$-in-a-row

Tesselation. Anallagmatic Problems . . . . . . 64

Colour-Cube Problem . . . . . . . . 67

Tangrams . . . . . . . . . . 69

Dynamical Games of Position . . . . . . . . . . 69

Shunting Problems . . . . . . . . . . 69

Ferry-Boat Problems . . . . . . . . . 71

Geodesic Problems . . . . . . . . . 73

Problems with Counters or Pawns . . . . . . . 74

Geometrical Puzzles with Rods. . . . . . . . . 80

Paradromic Rings . . . . . . . . . . . 80

Addendum on Solutiong . $\quad . \quad$. $\quad . \quad$. $\quad . \quad$. $\quad$. 81

Ohapter V. Mechanical Recreations.

Paradoses on Motion

Laws of Motion. . . . . . $\quad . \quad 87$

Force, Inertia. Centrifugal Force. . . . . . 87

Work. Stability of Equilibrium . • • • • • $\quad$ • 89

Perpetual Motion

Models

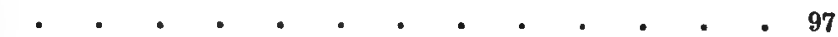

Sailing quioker than the wind. . . . . . . . . 98

Boat Moved by a Rope inside the Boat . . . . . . . 101

Hanksbee's Law . . . . . . . . . . . 101

Cut on Tennis-Balle, Cricket-Balls, Golf-Balls . . . . . 103

Flight of Birds . . . . . . . . . . . . 106

Ouriosa Physica, . • . . • . . . . . 107

Chapter Vi. Chess-Board Recreations.

Chess-Board Notation

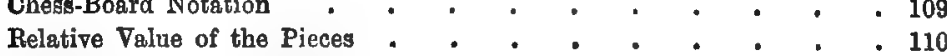

The Eight Queens Problem . . . . . . . . . 113

Masimum Pieces Problem. . . . . . . . . . 119

Minimum Pieces Problem . . . . . . . . . . 119

Analogoug Problem . . . . . . 121 
Re-Entrant Paths on a Chess-Board

Knight's Re-Entrant Path

King's Re-Entrant Path .

Rook's Re-Entrant Path.

Bishop's Re-Entrant Path

Misoellaneous Problems

Chapter Vil. Magic Squares.

Magic Squares and their History Construction of Odd Magic Squares

Construction of Singly-Even Magic Squares

Construction of Doubly-Eren Magic Squares .

Bordered Magic Squares

Number of Squares of certain Orders

Produot Squares

Magic Stars, eto.

Magic Pencils

Hyper-Magic Squares

Pandiagonal Squares

Symmetrical Squares

Doubly-Magio and Trebly-Magio Squares

Allied Magio Problems

Magic Card Square. Euler's Officers Problem

Magio Domino Squares .

Coin and 8tamp Squares

Chapter Vity. Bees and Their Cells.

The Form of Bees' Cells .

Economy Conjecture to explain the form . . . . . . 163

Mathematical Analysis of . . . . . . . . 164

Ellis's Suggestion to explain the form . . . . . . . 166

The Density Theory .

Chapter IX. Unicurgal Problems.

Euler's Problem.

Definitiong

Euler's Results

Illustrations

Number of Ways in whioh a Unicursal Figure can be described.

Rales for traversing a Maze.

Examples of Mazes. 
Chapter X. Kirrman's School-Girls Problem.

History of the Problem . . . . . . . . . . 193

Bolutions by One-Step Cycles . . . . . . . . . . 195

Examples when $n=3,9,27,33,51,57,75,81,99$. . . 196

Solutions by Two-Step Oycles . . . . . . . . . . 199

Examples when $n=15,27,89,51,63,75,87,99 \quad . \quad . \quad .200$

Solutions by Three-Step Cycles . . . . . . . . 203

Examples $n=9,21,27,33,39,45,51,57,63,69,75,81,87,92,99205$

Solutions by the Focal Method

Examples when $n=33,51 \quad . \quad$. . . . . . . 210

Analytioal Methods . . . . . . . . . . . 211

Application when $n=27$ with 13 as base . . . . . 212

Examples when $n=15,39$. . . . . . . . 216

Number of Solutions. . . . . . . . . . . 217

Harison's Theorem . . . . . . . . . . . 218

Problem of $n^{2}$ Girls in $n$ groups (Peirce) . . . . . . 219

Examples when $n=2,3,4,5,7,8 \quad$. $\quad . \quad . \quad . \quad . \quad 219$

Example when $n$ is prime . . . . . . . . 220

Kirkman's Problem in Quartets, dc. . . . . . . . 221

A Bridge Problem. Arrangements in Pairs . . . . . . 221

Bylvester's Corollary to Kirkman's Problem . . . . . . 222

Chapter XI. Miscellaneous Problems.

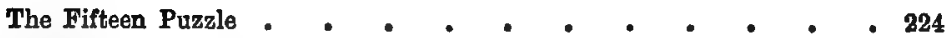

The Tower of Hanoí. $\quad . \quad+\quad . \quad+\quad . \quad . \quad . \quad \cdot \quad 228$

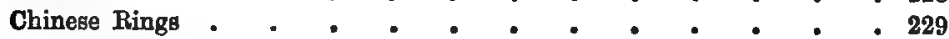

Algebraic Solntion . . . . . . . . . 230

Solution in Binary Scale of Notation . . . . . 232

Problems connected with a Pack of Carde . . . . . 234

Shuffling a Pack . . . . . . . . . . . 235

Arrangements by Rows and Columns . $\quad . \quad$. $. \quad . \quad$. $\quad 237$

Bachet's Problem with Pairs of Cards . . . . . - 238

The Three Pile Problem . . . . . . . . . . 240

Gergonne's Generalization . $\quad . \quad$..$\quad$. .241

The Mouse Trap. Treize - . - . - . • • • 245 
Chapter XII. Miscellaneous Problems (Continued). page Prefatory.

Arithmetical. Digit Questions. Two Examples . . . . . 247

Restorations, Class A. Four Examples . . . . . 248

Restorations, Class B. Five Examples . . . . . 249

Restorations, Class C. Two Examples . . . . . 252

Calendar Problems. Two Examples . . . . . . 253

Geometrical. The Five Disc Problem . . . . . . . 253

Tesselation with Super-Dominoes . . . . . . . 255

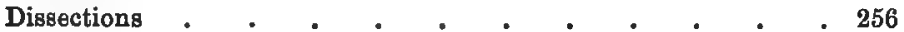

The Window Reader . . . . . . . . . 260

Compass Problems. . . . . . . . . . . . 262

Chapter XiII. Oalculating Prodigies.

Calculating Prodigies. Authorities . . . . . . . . 264

John Wallis, $1616-1703$. . . . . . . . . . 265

Buxton, circ. 1707-1772 . . . . . . . . . . 265

Problems solved by . . . . . . . . . 266

Methods of . . . . . . . . . . . . . . . 267

Fuller, 1710-1790 . . . . . . . . . . . . . . 268

Ampère, Gauss, Whately. . . . . • . . . . 268

Colburn, 1804-1840 . . . . . . . . . . . 269

Problems solved by. . . . . . . . . . . 269

Power of Factorizing Numbers . . . . . . . 270

Bidder, 1806-1878 • • . . . . • . • • . 271

Career of . . . . . . . . . . . . 271

Problems solved by . . . . . . . . . . 273

The Bidder Frmily . . . . . . . . . 275

Mondeux, Mangiamele . . . . . . . . . . 276

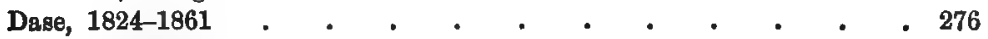

Problems solved by . . . . . . . . . . 277

Scientifio Work of . . . . . . . . . . 278

Safford, 1836-1901 • • • • • • • • • • . 278

Zamebone, Diamandi, Rückle . . . . . . . . . 279

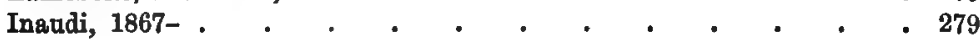

Problems solved by . . . . . . . . . 280

Expression of Numbers by Four Squares . . . . . 280

Nature of Public Performances . . . . . . . 280

Types of Memory of Numbers . . . . . . . . . 281

Bidder's Analysis of Methods used . . . . . . . . 282

Preferably only one step at a time . . . . . . 283

Multiplication. Examples of . . . . . . . 283

Division. Digit-Terminals . . . . . . . . 285

Digital Method for Division and Factors . . . . , 285

Square Roots. Higher Roots . . . . . . . 286

Compound Interest . . . . . . . . . 288

Logarithm . . . . . . . . . . . 289

Suggested Law of Rapidity of Caloulation . . . . . . 290

Requisitos for Arocoss . . , , . . . , , , 291 


\section{Chapter XIV. Cryptographs and Ciphers.}

Anthorities

Cryptographs. Definition. Dlustration. . . . 292

Ciphers. Definition. Illustration . . . . . . . . 293

Essential Features of Cryptographs and Ciphers . . . . . 293

Cryptographs of Three Types. . . . . . . . . 294

Transposition Type. The Route Method . . . . . 294

Use of non-significant symbols. The Grille . . . . 297

Use of broken symbols. The Scytale. . . . . . 299

Ciphers. Use of arbitrary symbols unnecessary . . . . . 300

Ciphers of Four Types . . . . . . . . . . . . 300

Substitution Alphabets. Illustrations. . . . . 300

Conrad's Tables of Frequency . . . . . . . 302

Ciphers of the Second Type. Illustrations . . . . . 303

Shifting Alphabets. Illustrations . . . . . . 306

Gronfeld's and the St Cyr Methods . . . . . . 306

The Playfair Cipher . . . . . . . . 308

Ciphers of the Fourth Type, Illustrations . . . . . 309

Desiderata in a good Cipher or Cryptograph . . . . . . 310

Cipher Machines . . . . . . . . . . . 311

On the Solution of Cryptographs and Ciphers . . . . . 311

Example . . . . . . . . . . 312

Historical Ciphers . . . . . . . . . . 315

Julius Caesar, Augustus, Bacon, Morse Code . . . . 315

Charles I . . . . . . . . . . 316

Pepys . . . . . . . . . . . . 317

De Rohan; Marie Antoinette. . . . . . . 318

The Fire Digit Code Dietionary . . . . . . 318

Chapter XV. String Figures.

A world-wide Amusement of Primitive Man . . . . . . 321

Construction of . . . . . . . . . . . . . . 321

Typical Examples of String Figures. . . . . . . . 322

A Mosquito or Fly (Amazon Indians) . . . . . . . . 322

A Man Climbing a Tree (Queensland Blacks) . . . . 323

Openings A, B. Novahoing . . . . . . . . , 324

$\Delta$ Tent Flap (Apache Red Indians) . . . . . . 325

Lightning (Navaho Indịans) . . • . . . . . 326

A Salmon Net (Caroline Islanders) . . . . . . 327

The Yam Thief (Gold Coast Negroes) . . . . . . 328

The Caterpillar (Polynesians) . . . . . . . 830

Cat's Oradle (Kores and Northern Europe). . . . , 881 
PAGE

A Dozen Typical String Tricks . . . . . . . . . $\quad$. 332

The Lizard Twist or Caroline Catch . . . . . . 332

The Chippewa Release . . . . . . . . . 333

Cheating the Halter. . . . . . . . . 333

The Threading of a Closed Loop . . . . . . . . 333

The Fly on the Nose . . . . . . . . . 334

The Interlaced Handcuffs . . . . . . . . . 335

The Button-Hole Trick . . . . . . . . . 335

The Waistcoat Trick . . . . . . . . . 336

\section{Chapter XVI. Three Classical Geometrical Problems.}

Statement of the Problems . . . . . . . . . 337

The Duplication of the Cube. Legendary Origin of the Problem . - 338

Hippocrates's Lemma . . . . . . . . . 340

Solutions by Archytas, Plato, Menaechmus, Apollonius, and Diocles . $\mathbf{3 4 0}$

Solutions by Vieta, Descartes, Gregory of St Vincent, and Newton - 343

The Trisection of an Angle . . . . . . . . . 344

Ancient Solutions quoted by Pappus . . . . . . . 344

Solutions by Pappus, Descartes, Newton, Clairaut, and Chasles . . 345

The Quadrature of the Circle . . . . . . . . . 346

Incommensurability of $\pi$. . . . . . . . . . . . 347

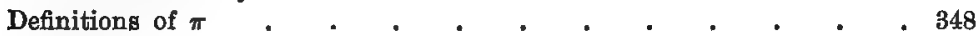

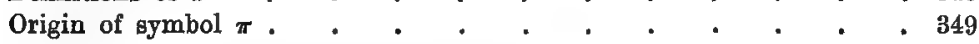

Methods of approximating to the numerical value of $\pi$. . . 349

Geometrical methods of Approximation . . . . . . . 349

Results of Egyptians, Babylonians, Jews . . . . . 350

Results of Archimedeg and other Greek Writers . . . . 350

Results of Roman Surveyors and Gerbert . . . . . 351

Results of Indian and Eastern Writers . . . . . 351

Results of European Writers, 1200-1630 . . . . . 353

Theorems of Wallis and Brouncker . . . . . . . 355

Analytical methods of Approximation. Gregory's series . . . 356

Results of European Writers, 1699-1873 . . . . . 356

Geometrical Approximation . . . . . . . . . 358

Approximations by the Theory of Probability $\quad \cdot \quad \cdot \quad \cdot \quad \cdot \quad 358$

Index.,$\ldots 60$ 

"Les hommes ne sont jamais plus ingénieux que dans l'invention des jeux, l'esprit s'y trouve à son aise... A près les jeux qui dépendent uniquement des nombres viennent les jeux où entre la situation.... Après les jeux où n'entrent que le nombre et la situation viendraient les jeux où entre le mouvement.... Enfin il serait à souhaiter qu'on eût un cours entier des jeux, traités mathématiquement." (Leibnitz: letter to De Montmort, July 29, 1715.) 


\section{CHAPTER I.}

\section{ARITHMETICAL RECREATIONS.}

I commence by describing some arithmetical recreations. The interest excited by statements of the relations between numbers of certain forms has been often remarked, and the majority of works on mathematical recreations include several such problems, which, though obvious to any one acquainted with the elements of algebra, have to many who are ignorant of that subject the same kind of charm that mathematicians find in the more recondite propositions of higher arithmetic. I devote the bulk of this chapter to these elementary problems.

Before entering on the subject, I may add that a large proportion of the elementary questions mentioned here are taken from one of two sources. The first of these is the classical Problèmes plaisans et délectables, by Claude Gaspar Bachet, sieur de Méziriac, of which the first edition was published in 1612 and the second in 1624 : it is to the edition of 1624 that the references hereafter given apply. Several of Bachet's problems are taken from the writings of Alcuin, Pacioli di Burgo, Tartaglia, and Cardan, and possibly some of them are of oriental origin, but I have made no attempt to add such references. The other source to which I alluded above is Ozanam's Récréations mathématiques et physiques. The greater portion of the original edition, published in two volumes at Paris in 1694, was a compilation from the works of Bachet, Mydorge, and Leurechon: this part is excellent, but the same cannot be said of the additions due to Ozanam. In the Biographie Universelle allusion is made to subsequent editions 
issued in 1720,1735, 1741, 1778, and 1790; doubtless these references are correct, but the following editions, all of which I have seen, are the only ones of which I have any knowledge. In 1696 an edition was issued at Amsterdam. In 1723-six years after the death of Ozanam-one was issued in three volumes, with a supplementary fourth volume, containing, among other things, an appendix on puzzles. Fresh editions were issued in 1741, 1750 (the second volume of which bears the date 1749), 1770, and 1790. The edition of 1750 is said to have been corrected by Montucla on condition that his name should not be associated with it; but the edition of 1790 is the earliest one in which reference is made to these corrections, though the editor is referred to only as Monsieur $\mathrm{M}^{* * *}$. Montucla expunged most of what was actually incorrect in the older editions, and added several historical notes, but unfortunately his scruples prevented him from striking out the accounts of numerous trivial experiments and truisms which overload the work. An English translation of the original edition appeared in 1708, and I believe ran through four editions, the last of them being published in Dublin in 1790 . Montucla's revision of 1790 was translated by C. Hutton, and editions of this were issued in 1803, in 1814, and (in one volume) in 1840: my references are to the editions of 1803 and 1840 .

I proceed to enumerate some of the typical elementary questions connected with numbers which for nearly three centuries have formed a large part of most compilatious of mathematical amusements. They are given here largely for their historical — not for their arithmetical-interest; and perhaps a mathematician may well omit this chapter.

Many of these questions are of the nature of tricks or puzzles, and $I$ follow the usual course and present them in that form. I may note however that most of them are not worth proposing, even as tricks, unless either the method employed is disguised or the result arrived at is different from that expected; but, as I am not writing on conjuring, I refrain from alluding to the 
means of disguising the operations indicated, and give merely a bare enumeration of the steps essential to the success of the method used. To the non-mathematician even to-day some of those results seem astonishing, but the secret is at once revealed as soon as the question is translated by symbols into mathematical language.

To FIND A NUMBER SELECTLD BY SOME ONE. There are innumerable ways of finding a number chosen by some one, provided the result of certain operations on it is known. I confine myself to methods typical of those commonly used. Any one acquainted with algebra will find no difficulty in framing new rules of an analogous nature.

First Method*. (i) Ask the person who has chosen the number to treble it. (ii) Enquire if the product is even or odd : if it is even, request him to take half of it; if it is odd, request him to add unity to it and then to take half of it. (iii) Tell him to multiply the result of the second step by 3 . (iv) Ask how many integral times 9 divides into the latter product: suppose the answer to be $n$. ( $\nabla$ ) Then the number thought of was $2 n$ or $2 n+1$, according as the result of step (i) was even or odd.

The demonstration is obvious. Every even number is of the form $2 n$, and the successive operations applied to this give (i) $6 n$, which is even; (ii) $\frac{1}{2} 6 n=3 n$; (iii) $3 \times 3 n=9 n$; (iv) $\frac{1}{9} 9 n=n$; (v) $2 n$. Every odd number is of the form $2 n+1$, and the successive operations applied to this give (i) $6 n+3$, which is odd; (ii) $\frac{1}{2}(6 n+3+1)=3 n+2$; (iii) $3(3 n+2)=9 n+6$; (iv) $\frac{1}{9}(9 n+6)=n+$ a remainder; (v) $2 n+1$. These results lead to the rule given above.

Second Method†. Ask the person who has chosen the number to perform in succession the following operations. (i) To multiply the number by 5 . (ii) To add 6 to the product. (iii) To multiply the sum by 4 . (iv) To add 9 to the product. (v) To multiply the sum by 5 . Ask to be told the result of the last operation: if from this product 165 is subtracted, and

\footnotetext{
* Bachet, Problèmes, Lyons, 1624, problem I, p. 53.

† A similar rule was given by Bachet, problem 17, p. 74.
} 
then the remainder is divided by 100 , the quotient will be the number thought of originally.

For let $n$ be the number selected. Then the successive operations applied to it give (i) $5 n$; (ii) $5 n+6$; (iii) $20 n+24$; (iv) $20 n+33$; (v) $100 n+165$. Hence the rule.

Third Method*. Request the person who has thought of the number to perform the following operations. (i) To multiply it by any number you like, say, $a$. (ii) To divide the product by any number, say, $b$. (iii) To multiply the quotient by c. (iv) To divide this result by $d$. (v) To divide the final result by the number selected originally. (vi) To add to the result of operation ( $v$ ) the number thought of at first. Ask for the sum so found: then, if $a c / b d$ is subtracted from this sum, the remainder will be the number chosen originally.

For, if $n$ was the number selected, the result of the first four operations is to form $n a c / b d$; operation (v) gives $a c / b d$; and (vi) gives $n+(a c / b d)$, which number is mentioned. But $a c / b d$ is known; hence, subtracting it from the number mentioned, $n$ is found. Of course $a, b, c, d$ may have any numerical values it is liked to assign to them. For example, if $a=12, b=4$, $c=7, d=3$ it is sufficient to subtract 7 from the final result in order to obtain the number originally selected.

Fourth Methodt. Ask some one to select a number less than 90. Request him to perform the following operations. (i) To multiply it by 10 , and to add any number he pleases, $a$, which is less than 10. (ii) To divide the result of step (i) by 3 , and to mention the remainder, say, $b$. (iii) To multiply the quotient obtained in step (ii) by 10 , and to add any number he pleases, $c$, which is less than 10. (iv) To divide the result of step (iii) by 3 , and to mention the remainder, say $d$, and the third digit (from the right) of the quotient; suppose this digit is $e$. Then, if the numbers $a, b, c, d, e$ are known, the original number can be at once determined. In fact, if the number is $9 x+y$, where $x \ngtr 9$ and $y \ngtr 8$, and if $r$ is the

- Baohet, problem v, p. 80.

$\dagger$ Educational Times, London, May 1, 1895, vol. ILvIr, p. 234. This example is said to have been made up by J. Clerk Maxwell in his boyhood: it is interesting to note how widely it differs from the simple Bachet problems previoubly mentioned. 
remainder when $a-b+3(c-d)$ is divided by 9 , we have $x=e, y=9-r$.

The demonstration is not difficult. Suppose the selected number is $9 x+y$. Step (i) gives $90 x+10 y+a$. Let $y+a=3 n+b$, then the quotient obtained in step (ii) is $30 x+3 y+n$. Step (iii) gives $300 x+30 y+10 n+c$. Let $n+c=3 m+d$, then the quotient obtained in step (iv) is $100 x+10 y+3 n+m$, which I will denote by $Q$. Now the third digit in $Q$ must be $x$, because, since $y \ngtr 8$ and $a \ngtr 9$, we have $n \ngtr 5$; and since $n \ngtr 5$ and $c \ngtr 9$, we have $m \ngtr 4$; therefore $10 y+3 n+m \ngtr 99$. Hence the third or hundreds digit in $Q$ is $x$.

Again, from the relations $y+a=3 n+b$ and $n+c=3 m+d$, we have $9 m-y=a-b+3(c-d):$ hence, if $r$ is the remainder when $a-b+3(c-d)$ is divided by 9 , we have $y=9-r$. [This is always true, if we make $r$ positive; but if $a-b+3(c-d)$ is negative, it is simpler to take $y$ as equal to its numerical value; or we may prevent the occurrence of this case by assigning proper values to $a$ and c.] Thus $x$ and $y$ are both known, and therefore the number selected, namely $9 x+y$, is known.

Fifth Method*. Ask any one to select a number less than 60 . Request him to perform the following operations. (i) To divide it by 3 and mention the remainder; suppose it to be $a$. (ii) To divide it by 4 , and mention the remainder; suppose it to be $b$. (iii) To divide it by 5 , and mention the remainder; suppose it to be $c$. Then the number selected is the remainder obtained by dividing $40 a+45 b+36 c$ by 60 .

This method can be generalized and then will apply to any number chosen. Let $a^{\prime}, b^{\prime}, c^{\prime}, \ldots$ be a series of numbers prime to one another, and let $p$ be their product. Let $n$ be any number less than $p$, and let $a, b, c, \ldots$ be the remainders when $n$ is divided by $a^{\prime}, b^{\prime}, c^{\prime}, \ldots$ respectively. Find a number $A$ which is a multiple of the product $b^{\prime} c^{\prime} d^{\prime} \ldots$ and which exceeds by unity a multiple of $a^{\prime}$. Find a number $B$ which is a multiple of $a^{\prime} c^{\prime} d^{\prime} \ldots$ and which exceeds by unity a multiple

- Bachet, problem $\nabla \mathrm{r}$, p. 84: Bachet added, on p. 87, a note on the previous history of the problem. 
of $b^{\prime}$, and similarly find analogous numbers $C, D, \ldots$ Rules for the calculation of $A, B, C, \ldots$ are given in the theory of numbers, but in general, if the numbers $a^{\prime}, b^{\prime}, c^{\prime}, \ldots$ are small, the corresponding numbers $A, B, C, \ldots$ can be found by inspection. I proceed to show that $n$ is equal to the remainder when $A a+B b+C c+\ldots$ is divided by $p$.

Let $N=A a+B b+C c+\ldots$, and let $M(x)$ stand for a multiple of $x$. Now $A=M\left(a^{\prime}\right)+1$, therefore $A a=M\left(a^{\prime}\right)+a$. Hence, if the first term in $N$, that is $A a$, is divided by $a^{\prime}$, the remainder is $a$. Again, $B$ is a multiple of $a^{\prime} c^{\prime} d^{\prime} \ldots$ Therefore $B b$ is exactly divisible by $a^{\prime}$ Similarly $C c, D d, \ldots$ are each exactly divisible by $a^{\prime}$. Thus every term in $N$, except the first, is exactly divisible by $a^{\prime}$. Hence, if $N$ is divided by $a^{\prime}$, the remainder is $a$. Also if $n$ is divided by $a^{\prime}$, the remainder is $a$.

Therefore

$$
\begin{aligned}
& N-n=M\left(a^{\prime}\right), \\
& N-n=M\left(b^{\prime}\right), \\
& N-n=M\left(c^{\prime}\right),
\end{aligned}
$$

Similarly

But $a^{\prime}, b^{\prime}, c^{\prime}, \ldots$ are prime to one another.

$$
\therefore N-n=M\left(a^{\prime} b^{\prime} c^{\prime} \ldots\right)=M(p) \text {, }
$$

that is,

$$
N=M(p)+n \text {. }
$$

Now $n$ is less than $p$, hence if $N$ is divided by $p$, the remainder is $n$.

The rule given by Bachet corresponds to the case of $a^{\prime}=3$, $b^{\prime}=4, c^{\prime}=5, p=60, A=40, B=45, C=36$. If the number chosen is less than 420 , we may take $a^{\prime}=3, b^{\prime}=4, c^{\prime}=5, d^{\prime}=7$, $p=420, A=280, B=105, C=336, D=120$.

To FIND THE RESUlT OF A SERIES OF OPERATIONS PERFORMED ON ANY NUMBER (unknown to the operator) WITHOUT ASKING ANY QUESTIONS. All rules for solving such problems ultimately depend on so arranging the operations that the number disappears from the final result. Four examples will suffice.

First Example*. Request some one to think of a number. Suppose it to be $n$. Ask him (i) to multiply it by any number you please, say, $a$; (ii) then to add, say, $b$; (iii) then to divide

* Bachet, problem vin, p. 102. 
the sum by, say, c. (iv) Next, tell him to take $a / c$ of the number originally chosen; and (v) to subtract this from the result of the third operation. The result of the first three operations is $(n a+b) / c$, and the result of operation (iv) is $n a / c$ : the difference between these is $b / c$, and therefore is known to you. For example, if $a=6, b=12, c=4$, then $a / c=1 \frac{1}{2}$, and the final result is 3 .

Second Example*. Ask $A$ to take any number of counters that he pleases: suppose that he takes $n$ counters. (i) Ask some one else, say $B$, to take $p$ times as many, where $p$ is any number you like to choose. (ii) Request $A$ to give $q$ of his counters to $B$, where $q$ is any number you like to select. (iii) Next, ask $B$ to transfer to $A$ a number of counters equal to $p$ times as many counters as $A$ has in his possession. Then there will remain in $B$ 's hands $q(p+1)$ counters: this number is known to you; and the trick can be finished either by mentioning it or in any other way you like.

The reason is as follows. The result of operation (ii) is that $B$ has $p n+q$ counters, and $A$ has $n-q$ counters. The result of (iii) is that $B$ transfers $p(n-q)$ counters to $A$ : hence he has left in his possession $(p n+q)-p(n-q)$ counters, that is, he has $q(p+1)$.

For example, if originally $\boldsymbol{A}$ took any number of counters, then (if you chose $p$ equal to 2), first you would ask $B$ to take twice as many counters as $A$ had done; next (if you chose $q$ equal to 3) you would ask $A$ to give 3 counters to $B$; and then you would ask $B$ to give to $A$ a number of counters equal to twice the number then in $A$ 's possession; after this was done you would know that $B$ had $3(2+1)$, that is, 9 left.

This trick (as also some of the following problems) may be performed equally well with one person, in which case $A$ may stand for his right hand and $B$ for his left hand.

Third Example. Ask some one to perform in succession the following operations. (i) Take any number of three digits, in which the difference between the first and last digits exceeds unity. (ii) Form a new number by reversing the order of

* Bachet, problem xilI, p. 123 : Bachet presented the above trick in a form, somewhat more general, but less effective in practice. 
the digits. (iii) Find the difference of these two numbers. (iv) Form another number by reversing the order of the digits in this difference. (v) Add together the results of (iii) and (iv). Then the sum obtained as the result of this last operation will be 1089 .

An illustration and the explanation of the rule are given below.

$\begin{array}{lcc}\text { (i) } & 237 & 100 a+10 b+c \\ \text { (ii) } & \frac{732}{495} & 100 c+10 b+a \\ \text { (iii) } & \frac{100(a-c-1)+90+(10+c-a)}{100(10+c-a)+90+(a-c-1)} \\ \text { (iv) } & \underline{\mathbf{5 9 4}} & \frac{1000+180+9}{\mathbf{1 0 8 9}} \\ \text { (v) } & \underline{ }\end{array}$

The result depends only on the radix of the scale of notation in which the number is expressed. If this radix is $r$, the result is $(r-1)(r+1)^{2}$; thus if $r=10$, the result is $9 \times 11^{2}$, that is, 1089 . Similar problems can be made with numbers exceeding 999.

Fourth Example*. The following trick depends on the same principle. Ask some one to perform in succession the following operations. (i) To write down any sum of money

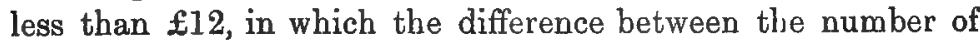
pounds and the number of pence exceeds unity. (ii) To reverse this sum, that is, to write down a sum of money obtained from it by interchanging the numbers of pounds and pence. (iii) To find the difference between the results of (i) and (ii). (iv) To reverse this difference. (v) To add together the results of (iii) and (iv). Then this sum will be £12. 18s. 11d.

For instance, take the sum $£ 10.17 s .5 d$; we have

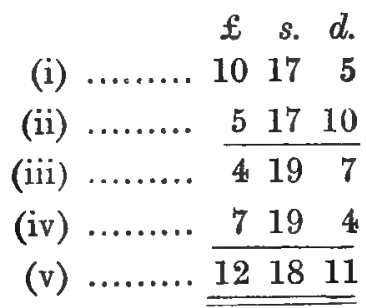

- Educational Times Reprints, 1890, vol. uni, p. 78. 
The following analysis explains the rule, and shows that the final result is independent of the sum written down initially.

\begin{tabular}{ccccc} 
(i) & & $\ldots$ & $s$. & $d$. \\
(ii) & $\ldots \ldots \ldots$ & $a$ & $b$ & $c$ \\
(iii) & $\ldots \ldots \ldots$ & $c$ & $b$ & $a$ \\
(iv) & $\ldots \ldots \ldots$ & $a-c-1$ & 19 & $c-a+12$ \\
(v) & $\ldots \ldots \ldots$ & $\frac{c-a+12}{11}$ & 19 & $a-c-1$ \\
\hline
\end{tabular}

Mr J. H. Schooling has used this result as the foundation of a slight but excellent conjuring trick. The rule can be generalized to cover any system of monetary units.

Problems involving Two Numbers. I proceed next to give a couple of examples of a class of problems which involve two numbers.

First Example*. Suppose that there are two numbers, one even and the other odd, and that a person $A$ is asked to select one of them, and that another person $B$ takes the other. It is desired to know whether $A$ selected the even or the odd number. Ask $A$ to multiply his number by 2 , or any even number, and $B$ to multiply bis by 3 , or any odd number. Request them to add the two products together and tell you the sum. If it is even, then originally $A$ selected the odd number, but if it is odd, then originally $A$ selected the even number. The reason is obvious.

Second Example $\uparrow$. The above rule was extended by Bachet to any two numbers, provided they are prime to one another and one of them is not itself a prime. Let the numbers be $m$ and $n$, and suppose that $n$ is exactly divisible by $p$. Ask $\boldsymbol{A}$ to select one of these numbers, and $B$ to take the other. Choose a number prime to $p$, say $q$. Ask $A$ to multiply his number by $q$, and $B$ to multiply his number by $p$. Request them to add the products together and state the sum. Then $A$ originally selected $m$ or $n$, according as this result is not or is divisible by $p$. The numbers, $m=7, n=15, p=3, q=2$, will illustrate the rest.

Problems depending on the Scale of Notation. Many of the rules for finding two or more numbers depend on the

* Bachet, problem Ix, p. $107 . \quad+$ Bachet, problem xI, p. 113. 
fact that in arithmetic an integral number is denoted by a succession of digits, where each digit represents the product of that digit and a power of ten, and the number is equal to the sum of these products. For example, 2017 signifies $\left(2 \times 10^{8}\right)+\left(0 \times 10^{2}\right)+(1 \times 10)+7$; that is, the 2 represents 2 thousands, i.e. the product of 2 and $10^{3}$, the 0 represents 0 hundreds, i.e. the product of 0 and $10^{2}$; the 1 represents 1 ten, i.e. the product of 1 and 10, and the 7 represents 7 units. Thus every digit has a local value. The application to tricks connected with numbers will be understood readily from three illustrative examples.

First Example*. A common conjuring trick is to ask a boy among the audience to throw two dice, or to select at random from a box a domino on each half of which is a number. The boy is then told to recollect the two numbers thus obtained, to choose either of them, to multiply it by 5 , to add 7 to the result, to double this result, and lastly to add to this the other number. From the number thus obtained, the conjurer subtracts 14, and obtains a number of two digits which are the two numbers chosen originally.

For suppose that the boy selected the numbers $a$ and $b$. Each of these is less than ten-dice or dominoes ensuring this. The successive operations give (i) $5 a$; (ii) $5 a+7$; (iii) $10 a+14$; (iv) $10 a+14+b$. Hence, if 14 is subtracted from the final result, there will be left a number of two digits, and these digits are the numbers selected originally. An analogous trick might be performed in other scales of notation if it was thought necessary to disguise the process further.

Second Example $\dagger$. Similarly, if three numbers, say, $a, b, c$, are chosen, then, if each of them is less than ten, they can be

- Some similar questions were given by Bachet in problem xn, p. 117; by Oughtred or Leake in the Mathematicall Recreations, commonly attributed to the former, London, 1653, problem xxxrv; and by Ozanam, part I, chapter $x$. Probably the Mathematicall Recreations were compiled by Leake, but as the work is usually catalogued under the name of W. Oughtred, I shall so describe it: it is founded on the similar work by J. Leureohon, otherwise known as H. $\operatorname{van}$ Etten, published in 1626 .

$\dagger$ Bachet gave some similar questions in problem $\mathbf{1 I}, \mathrm{p} .117$. 
found by the following rule. (i) Take one of the numbers, say, $a$, and multiply it by 2 . (ii) Add 3 to the product. (iii) Multiply this by 5 , and add 7 to the product. (iv) To this sum add the second number, $b$. (v) Multiply the result by 2. (vi) Add 3 to the product. (vii) Multiply by 5 , and, to the product, add the third number, $c$. The result is $100 a+10 b+c+235$. Hence, if the final result is known, it is sufficient to subtract 235 from it, and the remainder will be a number of three digits. These digits are the numbers chosen originally.

Third Example*. The following rule for finding the age of a man born in the 19th century is of the same kind. Take the tens digit of the year of birth; (i) multiply it by 5 ; (ii) to the product add 2; (iii) multiply the result by 2 ; (iv) to this product add the units digit of the birth-year; (v) subtract the sum from 120. The result is the man's age in 1916.

The algebraic proof of the rule is obvious. Let $a$ and $b$ be the tens and units digits of the birth-year. The successive operations give (i) $5 a$; (ii) $5 a+2$; (iii) $10 a+4$; (iv) $10 a+4+b$; (v) $120-(10 a+b)$, which is his age in 1916. The rule can be easily adapted to give the age in any specified year.

Fourth Examplef. Another such problem but of more difficulty is the determination of all numbers which are integral multiples of their reversals. For instance, among numbers of four digits, $8712=4 \times 2178$ and $9801=9 \times 1089$ possess this property.

Other Problems with numbers in the denary scale. I may mention here two or three other problems which seem to be unknown to most compilers of books of puzzles.

First Problem. The first of them is as follows. Take any number of three digits, the first and third digits being different: reverse the order of the digits: subtract the number so formed from the original number: then, if the last digit of the difference is mentioned, all the digits in the difference are known.

* A similar question was given by Laisant and Perrin in their Algèbre, Paris, 1892 ; and in L'Illustration for July 13, 1895.

† L'Intermédiaire des Mathématiciens, Paris, vol. xv, 1908, pp. 228, 278; vol. xvr, 1909, p. 34 ; vol. $\mathrm{xrx}, 1912$, p. 128. 
For suppose the number is $100 a+10 b+c$, then the number obtained by reversing the digits is $100 c+10 b+a$. The difference of these numbers is equal to $(100 a+c)-(100 c+a)$, that is, to $99(a-c)$. But $a-c$ is not greater than 9 , and therefore the remainder can only be $99,198,297,396,495,594,693,792$, or 891 ; in each case the middle digit being 9 and the digit before it (if any) being equal to the difference between 9 and the last digit. Hence, if the last digit is known, so is the whole of the remainder.

Second Problem. The second problem is somewhat similar and is as follows. (i) Take any number; (ii) reverse the digits; (iii) find the difference between the number formed in (ii) and the given number; (iv) multiply this difference by any number you like to name; (v) cross out any digit except a nought; (vi) read the remainder. Then the sum of the digits in the remainder subtracted from the next highest multiple of nine will give the figure struck out. This is clear since the result of operation (iv) is a multiple of nine, and the sum of the digits of every multiple of nine is itself a multiple of nine. This and the previous problem are typical of numerous analogous questions.

Empirical Problems. There are also numerous empirical problems, such as the following. With the ten digits, 9, 8, 7,6, $5,4,3,2,1,0$, express numbers whose sum is unity: each digit being used only once, and the use of the usual notations for fractions being allowed. With the same ten digits express numbers whose sum is 100 . With the nine digits, $9,8,7,6,5$, $4,3,2,1$, express numbers whose sum is 100 . To the making of such questions there is no limit, but their solution involves little or no mathematical skill.

Four Digits Problem. I suggest the following problem as being more interesting. With the digits $1,2,3$, 4, express the consecutive numbers from 1 upwards as far as possible: each of the four digits being used once and only once in the expression of each number. Allowing the notation of the denary scale (including decimals), as also algebraic sums, products, and positive integral powers, we can get to 88 . If the use of the symbols for square roots and factorials (repeated if desired a finite 
number of times) is also permitted, we can get to 264: if negative integral indices are also permitted, to 276 ; and if fractional indices are permitted, to 312. Many similar questions may be proposed, such as using four out of the digits $1,2,3,4,5$. With the five digits $1,2,3,4,5$, each being used once and only once, I have got to 3832 and 4282, according as negative and fractional indices are excluded or allowed.

Four Fours Problem. Another traditional recreation is, with the ordinary arithmetic and algebraic notation, to express the consecutive numbers from 1 upwards as far as possible in terms of four "4's." Everything turns on what we mean by ordinary notation. If $(\alpha)$ this is taken to admit only the use of the denary scale (ex. gr. numbers like 44), decimals, brackets, and the symbols for addition, subtraction, multiplication and division, we can thus express every number up to 22 inclusive. If $(\beta)$ also we grant the use of the symbol for square root (repeated if desired a finite number of times) we can get to 30 ; but note that though by its use a number like 2 can be expressed by one " 4 ," we cannot for that reason say that 2 is so expressible. If $(\gamma)$ further we permit the use of symbols for factorials we can express every number to 112. Finally, if $(\delta)$ we sanction the employment of integral indices expressible by a " 4 " or "4's" and allow the symbol for a square root to be used an infinite number of times we can get to 156 ; but if $(\epsilon)$ we concede the employment of integral indices and the use of sub-factorials* we can get to 877 . These interesting problems are typical of a class of similar questions. Thus, under conditions $y$ and using no indices, with four " 1 's" we can get to 34 , with four " 2 's" to 36 , with four " 3 's" to 46 , with four " 5 's" to 36 , with four " 6 's" to 30 , with four " 7 's" to 25 , with four " 8 's" to 36 , and with four " 9 's" to 66 .

Problems WITH a SERIES OF THINGS WHICH ARE NUMBERED. Any collection of things numbered consecutively lend themselves to easy illustrations of questions depending on elementary properties of numbers. As examples I proceed to enumerate a few familiar tricks. The first two of these are commonly shown

* Sub-factorial $n$ is equal to $n !(1-1 / 11+1 / 2 !-1 / 3 !+\ldots \pm 1 / n !)$. On the use of this for the four " 4 's" problem, see the Mathematical Gazette, May 1912. 
by the use of a watch, the last four may be exemplified by the use of a pack of playing cards.

First Example*. The first of these examples is connected with the hours marked on the face of a watch. In this puzzle some one is asked to think of some hour, say, $m$, and then to touch a number that marks another hour, say, $n$. Then if, beginning with the number touched, he taps each successive hour marked on the face of the watch, going in the opposite direction to that in which the hands of the watch move, and reckoning to himself the taps as $m,(m+1)$, \&c., the $(n+12)$ th tap will be on the hour he thought of. For example, if he thinks of $\nabla$ and touches IX, then, if he taps successively IX, VIII, VII, VI, .., going backwards and reckoning them respectively as $5,6,7,8, \ldots$, the tap which he reckons as 21 will be on the $v$.

The reason of the rule is obvious, for he arrives finally at the $(n+12-m)$ th hour from which he started. Now, since he goes in the opposite direction to that in which the hands of the watch move, he has to go over $(n-m)$ hours to reach the hour $m$ : also it will make no difference if in addition he goes over 12 hours, since the only effect of this is to take him once completely round the circle. Now $(n+12-m)$ is always positive, since $n$ is positive and $m$ is not greater than 12, and therefore if we make him pass over $(n+12-m)$ hours we can give the rule in a form which is equally valid whether $m$ is greater or less than $n$.

Second Example. The following is another well-known watch-dial problem. If the hours on the face are tapped successively, beginning at VII and proceeding backwards round the dial to VI, V, \&c., and if the person who selected the number counts the taps, beginning to count from the number of the hour selected (thus, if he selected $\mathbf{x}$, he would reckon the first tap as the 11th), then the 20th tap as reckoned by him will be on the hour chosen.

For suppose he selected the $n$th hour. Then the 8th tap is on XII and is reckoned by him as the $(n+8)$ th ; and the tap

* Bachet, problem xx, p. 155; Oughtred or Leake, Mathematicall Recrecttions, London, 1653, p. 28. 
which he reckons as $(n+p)$ th is on the hour $(20-p)$. Hence, putting $p=20-n$, the tap which he reckons as 20th is on the hour $n$. Of course the hours indicated by the first seven taps are immaterial: obviously also we can modify the presentation by beginning on the hour VIII and making 21 consecutive taps, or on the hour IX and making 22 consecutive taps, and so on.

Third Example. The following is another simple example. Suppose that a pack of $n$ cards is given to some one who is asked to select one out of the first $m$ cards and to remember (but not to mention) what is its number from the top of the pack; suppose it is actually the $x$ th card in the pack. Then take the pack, reverse the order of the top $m$ cards (which can be easily effected by shuffling), and transfer $y$ cards, where $y<n-m$, from the bottom to the top of the pack. The effect of this is that the card originally chosen is now the $(y+m-x+1)$ th from the top. Return to the spectator the pack so rearranged, and ask that the top card be counted as the $(x+1)$ th, the next as the $(x+2)$ th, and so on, in which case the card originally chosen will be the $(y+m+1)$ th. Now $y$ and $m$ can be chosen as we please, and may be varied every time the trick is performed; thus any one unskilled in arithmetic will not readily detect the method used.

Fourth Example*. Place a card on the table, and on it place as many other cards from the pack as with the number of pips on the card will make a total of twelve. For example, if the card placed first on the table is the five of clubs, then seven additional cards must be placed on it. The court cards may have any values assigned to them, but usually they are reckoned as tens. This is done again with another card, and thus another pile is formed. The operation may be repeated either only three or four times or as often as the pack will permit of such piles being formed. If finally there are $p$ such piles, and if the number of cards left over is $r$, then the sum of the number of pips on the bottom cards of all the piles will be $13(p-4)+r$.

For, if $x$ is the number of pips on the bottom card of a pile,

- A particular case of this problem was given by Bachet, problem xvir, p. 138. 
the number of cards in that pile will be $13-x$. A similar argument holds for each pile. Also there are 52 cards in the pack; and this must be equal to the sum of the cards in the $p$ piles and the $r$ cards left over.

$$
\begin{array}{r}
\therefore\left(13-x_{1}\right)+\left(13-x_{2}\right)+\ldots+\left(13-x_{p}\right)+r=52, \\
\therefore 13 p-\left(x_{1}+x_{2}+\ldots+x_{p}\right)+r=52, \\
\therefore x_{1}+x_{2}+\ldots+x_{p}=13 p-52+r \\
=13(p-4)+r .
\end{array}
$$

More generally, if a pack of $n$ cards is taken, and if in each pile the sum of the pips on the bottom card and the number of cards put on it is equal to $m$, then the sum of the pips on the bottom cards of the piles will be $(m+1) p+r-n$. In an écarté pack $n=32$, and it is convenient to take $m=15$.

Fifth Example. It may be noticed that cutting a pack of cards never alters the relative position of the cards provided that, if necessary, we regard the top card as following immediately after the bottom card in the pack. This is used in the following trick*. Take a pack, and deal the cards face upwards on the table, calling them one, two, three, \&c. as you put them down, and noting in your own mind the card first dealt. Ask some one to select a card and recollect its number. Turn the pack over, and let it be cut (not shuffled) as often as you like. Enquire what was the number of the card chosen. Then, if you deal, and as soon as you come to the original first card begin (silently) to count, reckoning this as one, the selected card will appear at the number mentioned. Of course, if all the cards are dealt before reaching this number, you must turn the cards over and go on counting continuously.

Sixth Example. Here is another simple question of this class. Remove the court cards from a pack. Arrange the remaining 40 cards, faces upwards, in suits, in four lines thus. In the first line, the $1,2, \ldots 10$, of suit $A$; in the second line, the $10,1,2, \ldots 9$, of suit $B$; in the third line, the $9,10,1, \ldots 8$, of suit $C$; in the last line, the $8,9,10,1, \ldots 7$, of suit $D$. Next take up, face upwards, the first-card of line 1 , put below it the

- Bschet, problem xix, p. 152. 
tirst card of line 2, below that the first card of line 3 , and below that the first card of line 4. Turn this pile face downwards. Next take up the four cards in the second column in the same way, turn them face downwards, and put them below the first pile. Continue this process until all the cards are taken up. Ask someone to mention any card. Suppose the number of pips on it is $n$. Then if the suit is $A$, it will be the $4 n$th card in the pack; if the suit is $B$, it will be the $(4 n+3)$ th card; if the suit is $C$, it will be the $(4 n+6)$ th card ; and if the suit is $D$, it will be the $(4 n+9)$ th card. Hence by counting the cards, cyclically if necessary, the card desired can be picked out. It is easy to alter the form of presentation, and a full pack can be used if desired. The explanation is obvious.

Medieval Problems in Arithmetic. Before leaving the subject of these elementary questions, I may mention a few problems which for centuries have appeared in nearly every collection of mathematical recreations, and therefore may claim what is almost a prescriptive right to a place here.

First Example. The following is a sample of one class of these puzzles. A man goes to a tub of water with two jars, of which one holds exactly 3 pints and the other 5 pints. How can he bring back exactly 4 pints of water? The solution presents no difficulty.

Second Example*. Here is another problem of the same kind. Three men robbed a gentleman of a vase, containing 24 ounces of balsam. Whilst running away they met a glass-seller, of whom they purchased three vessels. On reaching a place of safety they wished to divide the booty, but found that their vessels contained 5,11 , and 13 ounces respectively. How could they divide the balsam into equal portions? Problems like this can be worked out only by trial.

Third Examplet. The next of these is a not uncommon

* Some similar problems were given by Bachet, Appendix, problem II, p. 206; problem Ix, p. 233: by Oughtred or Leake in the Mathematicall Recreations, p. 22: and by Ozanam, 1803 edition, vol. I, p. 174 ; 1840 edition, p. 79. Earlier instances occur in Tartaglia's writings.

+ Bachet, problem xxII, p. 170. 
game, played by two people, say $A$ and $B$. $A$ begins by mentioning some number not greater than (say) six, $B$ may add to that any number not greater than six, $A$ may add to that again any number not greater than six, and so on. He wins who is the first to reach (say) 50. Obviously, if $A$ calls 43 , then whatever $B$ adds to that, $A$ can win next time. Similarly, if $A$ calls $36, B$ cannot prevent $A$ 's calling 43 the next time. In this way it is clear that the key numbers are those forming the arithmetical progression 43, 36, 29, 22, 15, 8,1 ; and whoever plays first ought to win.

Similarly, if no number greater than $m$ may be added at any one time, and $n$ is the number to be called by the victor, then the key numbers will be those forming the arithmetical progression whose common difference is $m+1$ and whose smallest term is the remainder obtained by dividing $n$ by $m+1$.

The same game may be played in another form by placing $p$ coins, matches, or other objects on a table, and directing each player in turn to take away not more than $m$ of them. Whoever takes away the last coin wins. Obviously the key numbers are multiples of $m+1$, and the first player who is able to leave an exact multiple of $(m+1)$ coins can win. Perhaps a better form of the game is to make that player lose who takes away the last coin, in which case each of the key numbers exceeds by unity a multiple of $m+1$.

Another variety* consists in placing $p$ counters in the form of a circle, and allowing each player in succession to take away not more than $m$ of them which are in unbroken sequence: $m$ being less than $p$ and greater than unity. In this case the second of the two players can always win.

These games are simple, but if we impose on the original problem the restriction that each player may not add the same number more than (say) three times, the analysis becomes by no means easy. I have never seen this extension described in print, and I will enunciate it at length. Suppose that each player is given eighteen cards, three of them marked 6 , three marked 5 , three marked 4 , three 
marked 3, three marked 2, and three marked 1 . They play alternately; $A$ begins by playing one of his cards; then $B$ plays one of his, and so on. He wins who first plays a card which makes the sum of the points or numbers on all the cards played exactly equal to 50, but he loses if be plays a card which makes this sum exceed 50 . The game can be played by noting the numbers on a piece of paper, and it is not necessary to use cards.

Thus suppose they play as follows. $A$ takes a 4 , and scores 4; $B$ takes a 3 , and scores $7 ; A$ takes a 1 , and scores $8 ; B$ takes a 6 , and scores $14 ; A$ takes a 3 , and scores $17 ; B$ takes a 4 , and scores $21 ; A$ takes a 4 , and scores $25 ; B$ takes a 5 , and scores $30 ; A$ takes a 4 , and scores $34 ; B$ takes a 4 , and scores $38 ; A$ takes a 5 , and scores 43 . $B$ can now win, for he may safely play 3 , since $A$ has not another 4 wherewith to follow it; and if $A$ plays less than $4, B$ will win the next time. Again, suppose they play thus. $A, 6 ; B, 3 ; A, 1 ; B, 6 ; A, 3 ; B, 4 ; A, 2$; $B, 5 ; A, 1 ; B, 5 ; A, 2 ; B, 5 ; A, 2 ; B, 3 . A$ is now forced to play 1 , and $B$ wins by playing 1 .

A slightly different form of the game has also been suggested. In this there are put on the table an agreed number of cards, say, for example, the four aces, twos, threes, fours, fives, and sixes of a pack of cards-twenty-four cards in all. Each player in turn takes a card. The score at any time is the sum of the pips on all the cards taken, whether by $A$ or $B$. He wins who first selects a card which makes the score equal, say, to 50 , and a player who is forced to go beyond 50 loses.

Thus, suppose they play as follows. $A$ takes a 6 , and scores $6 ; B$ takes a 2 , and scores $8 ; A$ takes a 5 , and scores 13 ; $B$ takes a 2 , and scores $15 ; A$ takes a 5 , and scores $20 ; B$ takes a 2 , and scores $22 ; A$ takes a 5 , and scores $27 ; B$ takes a 2 , and scores $29 ; A$ takes a 5 , and scores $34 ; B$ takes a 6 , and scores $40 ; A$ takes a 1 , and scores $41 ; B$ takes a 4 , and scores $45 ; A$ takes a 3 , and scores $48 ; B$ now must take 1 , and thus score 49 ; and $A$ takes a 1 , and wins.

In these variations the object of each player is to get to one of the key numbers, provided there are sufficient available 
remaining numbers to let him retain the possession of each subsequent key number. The number of cards used, the points on them, and the number to be reached can be changed at will; and the higher the number to be reached, the more difficult it is to forecast the result and to say whether or not it is an advantage to begin.

Fourth Example. Here is another problem, more difficult and less well known. Suppose that $m$ counters are divided into $n$ heaps. Two players play alternately. Each, when his turn comes, may select any one heap he likes, and remove from it all the counters in it or as many of them as he pleases. That player loses who has to take up the last counter.

To solve it we may proceed thus*. Suppose there are $a_{r}$ counters in the $r$ th heap. Express $a_{r}$ in the binary scale, and denote the coefficient of $2^{p}$ in it by $d_{r p}$. Do this for each heap, and let $S_{p}$ be the sum of the coefficients of $2^{p}$ thus determined. Thus $S_{p}=d_{1 p}+d_{p p}+d_{3 p}+\ldots$ Then either $S_{0}$, $S_{1}, S_{2}, \ldots$ are all even, which we may term an $A$ arrangement, or they are not all even, which we may term a $B$ arrangement.

It will be easily seen that if one player, $P$, has played so as to get the counters in any $A$ arrangement (except that of an even number of heaps each containing one counter), he can force a win. For the next move of his opponent, $Q$, must bring the counters to a $B$ arrangement. Then $P$ can make the next move to bring the counters again to an $A$ arrangement, other than the exceptional one of an even number of heaps each containing only one counter. Finally this will leave $P$ a winning position, ex. gr. two heaps each containing 2 counters.

If that player wins who takes the last counter, the rule is similar. For if one player $P$ has played so as to get the counters in any $A$ arrangement, including therein an even number of heaps each containing one counter, he can force a win, since the next move of his opponent $Q$ must bring the counters to a $B$ arrangement. Then $P$ can make the next move to bring the counters again to an $A$ arrangement. Finally this will leave $P$ a winning position.

Fifth Example. The following medieval problem is somewhat

- C. I. Bouton, Nim, Annals of Mathematics, Harvard, U.S.A., Beries 2, vol. w1, 1901-02, pp. 35-39. 
more elaborate. Suppose that three people, $P, Q, R$, select three things, which we may denote by $a, e, i$ respectively, and that it is desired to find by whom each object was selected*.

Place 24 counters on a table. Ask $P$ to take one counter, $Q$ to take two counters, and $R$ to take three counters. Next, ask the person who selected $a$ to take as many counters as he has already, whoever selected $e$ to take twice as many counters as he has already, and whoever selected $i$ to take four times as many counters as he has already. Note how many counters remain on the table. There are only six ways of distributing the three things among $P, Q$, and $R$; and the number of counters remaining on the table is different for each way. The remainders may be $1,2,3,5,6$, or 7 . Bachet summed up the results in the mnemonic line Par fer (1) César (2) jadis (3) devint (5) si grand (6) prince (7). Corresponding to any remainder is a word or words containing two syllables: for instance, to the remainder 5 corresponds the word devint. The vowel in the first syllable indicates the thing selected by $P$, the vowel in the second syllable indicates the thing selected by $Q$, and of course $R$ selected the remaining thing.

Extension. M. Bourlet, in the course of a very kindly notice $\dagger$ of the second edition of this work, gave a much neater solution of the above question, and has extended the problem to the case of $n$ people, $P_{0}, P_{1}, P_{2}, \ldots, P_{n-1}$, each of whom selects one object, out of a collection of $n$ objects, such as dominoes or cards. It is required to know which domino or card was selected by each person.

Let us suppose the dominoes to be denoted or marked by the numbers $0,1, \ldots, n-1$, instead of by vowels. Give one counter to $P_{1}$, two counters to $P_{2}$, and generally $k$ counters to $P_{k}$. Note the number of counters left on the table. Next ask the person who had chosen the domino 0 to take as many counters as he had already, and generally whoever had chosen the domino $h$ to take $n^{h}$ times as many dominoes as he had already: thus if $P_{h}$ had chosen the domino numbered $h$,

* Bachet, problem xxv, p. 187.

† Bulletin des Sciences Mathématiques, Paris, 1893, vol. xvI, pp. 105-107. 
he would take $n^{h} k$ counters. The total number of counters taken is $\Sigma n^{h} k$. Divide this by $n$, then the remainder will be the number on the domino selected by $P_{0}$; divide the quotient by $n$, and the remainder will be the number on the domino selected by $P_{1}$; divide this quotient by $n$, and the remainder will be the number on the domino selected by $P_{2}$; and so on. In other words, if the number of counters taken is expressed in the scale of notation whose radix is $n$, then the $(h+1)$ th digit from the right will give the number on the domino selected by $P_{h}$.

Thus in Bachet's problem with 3 people and 3 dominoes, we should first give one counter to $Q$, and two counters to $R$, while $P$ would have no counters; then we should ask the person who had selected the domino marked 0 or $a$ to take as many counters as he had already, whoever had selected the domino marked 1 or $e$ to take three times as many counters as he had already, and whoever had selected the domino marked 2 or $i$ to take nine times as many counters as he had already. By noticing the original number of counters, and observing that 3 of these had been given to $Q$ and $R$, we should know the total number taken by $P, Q$, and $R$. If this number were divided by 3 , the remainder would be the number of the domino chosen by $P$; if the quotient were divided by 3 the remainder would be the number of the domino chosen by $Q$; and the final quotient would be the number of the domino chosen by $R$.

Exploration Problems. Another common question is concerned with the maximum distance into a desert which could be reached from a frontier settlement by the aid of a party of $n$ explorers, each capable of carrying provisions that would last one man for $a$ days. The answer is that the man who reaches the greatest distance will occupy $n a /(n+1)$ days before he returns to his starting point. If in the course of their journey they may make depôts, the longest possible journey will occupy $\frac{1}{2} a\left(1+\frac{1}{2}+\frac{1}{3}+\ldots+1 / n\right)$ days.

The Josephus Problem. Another of these antique problems consists in placing men round a circle so that if every $m$ th man is killed, the remainder shall be certain specified individuals. Such problems can be easily solved empirically. 
Hegesippus* says that Josephus saved his life by such a device. According to his account, after the Romans had captured Jotapat, Josephus and forty other Jews took refuge in a cave. Josephus, much to his disgust, found that all except himself and one other man were resolved to kill themselves, so as not to fall into the hands of their conquerors. Fearing to show his opposition too openly he consented, but declared that the operation must be carried out in an orderly way, and suggested that they should arrange themselves round a circle and that every third person should be killed until but one man was left, who must then commit suicide. It is alleged that he placed himself and the other man in the 31st and 16th place respectively.

The medieval question was usually presented in the following form. A ship, carrying as passengers 15 Turks and 15 Christians, encountered a storm, and, in order to save the ship and crew, one-half of the passengers had to be thrown into the sea. Accordingly the passengers were placed in a circle, and every ninth man, reckoning from a certain point, was cast overboard. It is desired to find an arrangement by which all the Christians should be saved $\dagger$. In this case we must arrange the men thus: CCCCTTTTTCCTCCCTCT TCCT T TCT TCCT, where $C$ stands for a Christian and $T$ for a Turk. The order can be recollected by the positions of the vowels in the following line: From numbers' aid and art, never will fame depart, where $a$ stands for $1, e$ for $2, i$ for $3, o$ for 4 , and $u$ for 5 . Hence the order is $o$ Christians, $u$ Turks, \&c.

If every tenth man were cast overboard, a similar mnemonic line is Rex paphi cum gente bona dat signa serena. An oriental setting of this decimation problem runs somewhat as follows. Once upon a time, there lived a rich farmer who had 30 children, 15 by his first wife who was dead, and 15 by his second wife. The latter woman was eager that her eldest son should inherit the property. Accordingly one day she said to him, "Dear Husband,

- De Bello Judaico, bk. IIr, chaps. 16-18.

+ Bachet, problem xxm, p. 174. The same problem had been previously enunciated by Tartaglia. 
you are getting old. We ought to settle who shall be your heir. Let us arrange our 30 children in a circle, and counting from one of them remove every tenth child until there remains but one, who shall succeed to your estate." The proposal seemed reasonable. As the process of selection went on, the farmer grew more and more astonished as he noticed that the first 14 to disappear were children by his first wife, and he observed that the next to go would be the last remaining member of that family. So he suggested that they should see what would happen if they began to count backwards from this lad. She, forced to make an immediate decision, and reflecting that the odds were now 15 to 1 in favour of her family, readily assented. Who became the heir?

In the general case $n$ men are arranged in a circle which is closed up as individuals are picked out. Beginning anywhere, we continually go round, picking out each $m$ th man until only $r$ are left. Let one of these be the man who originally occupied the $p$ th place. Then had we begun with $n+1$ men, he would have originally occupied the $(p+m)$ th place when $p+m$ is not greater than $n+1$, and the $(p+m-n-1)$ th place when $p+m$ is greater than $n+1$. Thus, provided there are to be $r$ men left, their original positions are each shifted forwards along the circle $m$ places for each addition of a single man to the original group*.

Now suppose that with $n$ men the last survivor $(r=1)$ occupied originally the $p$ th place, and that with $(n+x)$ men the last survivor occupied the $y$ th place. Then, if we confine ourselves to the lowest value of $x$ which makes $y$ less than $m$, we have $y=(p+m x)-(n+x)$.

Based on this theorem we can, for any specified value of $n$, calculate rapidly the position occupied by the last survivor of the company. In effect, Tait found the values of $n$ for which a man occupying a given position $p$, which is less than $m$, would be the last survivor, and then by repeated applications of the proposition, obtained the position of the survivor for intermediate values of $n$.

* P. G. Tait, Collected Scientific Papers, Cambridge, vol. n. 1900, pp. 432-435. 
For instance, take the Josephus problem in which $m=3$. Then we know that the final survivor of 41 men occupied originally the 31st place. Suppose that when there had been $(41+x)$ men, the survivor occupied originally the $y$ th place. Then, if we consider only the lowest value of $x$ which makes $y$ less than $m$, we have $y=(31+3 x)-(41+x)=2 x-10$. Now, we have to take a value of $x$ which makes $y$ positive and less than $m$, that is, in this case equal to 1 or 2 . This is $x=6$ which makes $y=2$. Hence, had there been 477 men the man last chosen would have originally occupied the second place. Similarly had there been $(47+x)$ men the man would have occupied originally the $y$ th place, where, subject to the same conditions as before, we have $y=(2+3 x)-(47+x)=2 x-45$. If $x=23, y=1$. Hence, with 70 men the man last chosen would have occupied originally the first place. Continuing the process, it is easily found that if $n$ does not exceed 2000000 the last man to be taken occupies the first place when $n=4,6,9,31,70,105,355,799,1798,2697,9103,20482,30723$, $69127,155536,233304,349956,524934$, or 787401 ; and the second place when $n=2,3,14,21,47,158,237,533,1199,4046$, $6069,13655,46085,103691,1181102$, or 1771653 . From these results, by repeated applications of the proposition, we find, for any intermediate values of $n$, the position originally occupied by the man last taken. Thus with 1000 men, the 604th place; with 100000 men, the 92620th place; and with 1000000 men, the 637798th place are those which would be selected by a prudent mathematician in a company subjected to trimation.

Similarly if a set of 100 men were subjected to decimation, the last to be taken would be the man originally in the 26th place. Hence, with 227 men the last to be taken would be the man originally in the first place.

Modifications of the original problem have been suggested. For instance*, let 5 Christians and 5 Turks be arranged round a circle thus, TCTCCTCTCT. Suppose that, if beginning at the ath man, every $h$ th man is selected, all the Turks will be picked out for punishment; but if beginning at the bth man,

* H. E. Dudeney, Tit-Bits, London, Oct. 14 and 28, 1905. 
every $k$ th man is selected, all the Christians will be picked out for punishment. The problem is to find $a, b, h$, and $k$. A solution is $a=1, h=11, b=9, k=29$.

I suggest as a similar problem, to find an arrangement of $c$ Turks and $c$ Christians arranged in a circle, so that if beginning at a particular man, say the first, every $h$ th man is selected, all the Turks will be picked out, but if, beginning at the same man, every $k$ th man is selected, all the Christians will be picked out. This makes an interesting question because it is conceivable that the operator who picked out the victims might get confused and take $k$ instead of $h$, or vice versa, and so consign all his friends to execution instead of those whom he had intended to pick out. The problem is, for any given value of $c$, to find an arrangement of the men and the corresponding suitable values of $h$ and $k$. Obviously if $c=2$, then for an arrangement like $T C C T$ a solution is $h=4, k=3$. If $c=3$, then for an arrangement like $T C T C C T$ a solution is $h=7, k=8$. If $c=4$, then for an arrangement like TCTTCTCC a solution is $h=9$, $k=5$. And generally, as first pointed out by $\mathrm{Mr}$ Swinden, with $2 c$ men, $c$ of them, occupying initially the consecutive places numbered $c, c+1, \ldots, 2 c-1$, will be picked out, if $h$ is the L.C.M. of $c+1, c+2, \ldots, 2 c-1$; and the other $c$ will be picked out if $k=h+1$, though it may well be that there is a simpler solution for another initial arrangement. It may be impossible to arrange the men so that $n$ specified individuals shall be picked out in a defined order.

\section{ADDENDUM.}

Note. Page 13. Solutions of the ten digit problems are $35 / 70+148 / 296=1$, or $\cdot 01234+\cdot 98765=1$; and $50+49+1 / 2+38 / 76=100$. A solution of the nine digit problem is $1 \cdot 234+98 \cdot 765=100$, or $97+8 / 12+4 / 6+5 / 3=100$; but if an algebraic sum is permissible a neater solution is $123-45-67+89=100$, where the digits occur in their natural order.

Note. Page 18. There are several solutions of the division of 24 ounces under the conditions specified. One of these solutions is as follows :

The vessels can contain........ $24 \mathrm{oz}, 13 \mathrm{oz} .11 \mathrm{oz} .5 \mathrm{oz}$.

$\begin{array}{lrrrr}\text { Their contents originally are... } 24 \ldots & 0 \ldots & 0 \ldots & 0 \ldots\end{array}$

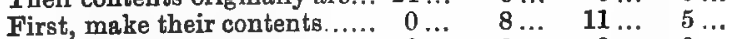

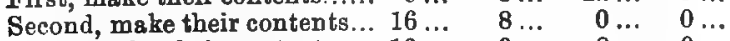

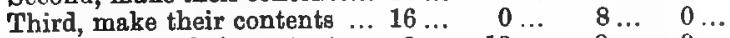

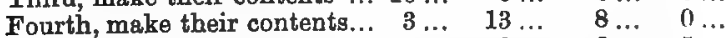

$\begin{array}{llllll}\text { Fifth, make their contents...... } & 3 \ldots & 8 \ldots & 8 \ldots & 5 \ldots\end{array}$

Lastly, make their contents ... 8 ... 8 8... 8 8... $0 .$. 


\section{CHAPTER II.}

\section{ARITHMETICAL RECREATIONS CONTINUED.}

I devote this chapter to the description of some arithmetical fallacies, a few additional problems, and notes on one or two problems in higher arithmetic.

ARithmetical FALLACIES. I begin by mentioning some instances of demonstrations* leading to arithmetical results which are obviously impossible. I include algebraical proofs as well as arithmetical ones. Some of the fallacies are so patent that in.preparing the first and second editions I did not think such questions worth printing, but, as some correspondents expressed a contrary opinion, I give them for what they are worth.

First Fallacy. One of the oldest of these-and not a very interesting specimen-is as follows. Suppose that $a=b$, then

$$
\begin{gathered}
a b=a^{2} . \quad \therefore a b-b^{2}=a^{2}-b^{2} . \quad \therefore b(a-b)=(a+b)(a-b) . \\
\therefore b=a+b . \quad \therefore b=2 b . \quad \therefore 1=2 .
\end{gathered}
$$

* Of the fallacies given in the text, the first and second are well known; the third is not new, but the earliest work in which I recollect seeing it is my Algebra, Cambridge, 1890, p. 430; the fourth is given in G. Chrystal's Algebra, Edinburgh, 1889, vol. $\pi$, p. 159 ; the sixth is due to G. T. Walker, and, I believe, has not appeared elsewhere than in this book; the seventh is due to D'Alembert; and the eighth to F. Galton. It may be worth recording (i) that a mechanical demonstration that $1=2$ was given by $R$. Chartres in Knowledge, July, 1891; and (ii) that J. L. F. Bertrand pointed out that a demonstration that $1=-1$ can be obtained from the proposition in the Integral Calculus that, if the limits are constant, the order of integration is indifferent; hence the integral to $x$ (from $x=0$ to $x=1$ ) of the integral to $y$ (from $y=0$ to $y=1$ ) of a function $\phi$ should be equal to the integral to $y$ (trom $y=0$ to $y=1$ ) of the integral to $x$ (from $x=0$ to $x=1$ ) of $\phi$, but if $\phi=\left(x^{2}-y^{2}\right) /\left(x^{2}+y^{2}\right)^{2}$, this gives $\frac{1}{6} \pi=-\frac{1}{2} \pi$. 
Second Fallacy. Another example, the idea of which is due to John Bernoulli, may be stated as follows. We have $(-1)^{2}=1$. Take logarithms, $\therefore 2 \log (-1)=\log 1=0 . \quad \therefore \log (-1)=0$. $\therefore-1=e^{0} . \quad \therefore-1=1$.

The same argument may be expressed thus. Let $x$ be a quantity which satisfies the equation $e^{x}=-1$. Square both sides,

$$
\therefore e^{2 x}=1 . \quad \therefore 2 x=0 . \quad \therefore x=0 . \quad \therefore e^{x}=e^{0} \text {. }
$$

But $e^{x}=-1$ and $e^{0}=1, \therefore-1=1$.

The error in each of the foregoing examples is obvious, but the fallacies in the next examples are concealed somewhat better.

Third Fallacy. As yet another iustance, we know that

$$
\log (1+x)=x-\frac{1}{2} x^{2}+\frac{1}{3} x^{3}-\ldots .
$$

If $x=1$, the resulting series is convergent; hence we have $\log 2=1-\frac{1}{2}+\frac{1}{8}-\frac{1}{4}+\frac{1}{5}-\frac{1}{6}+\frac{1}{8}-\frac{1}{8}+\frac{1}{9}-\ldots$.

$$
\therefore 2 \log 2=2-1+\frac{9}{3}-\frac{1}{2}+\frac{2}{5}-\frac{1}{3}+\frac{2}{4}-\frac{1}{4}+\frac{2}{9}-\ldots .
$$

Taking those terms together which have a common denominator, we obtain

Hence

$$
\begin{aligned}
2 \log 2 & =1+\frac{1}{3}-\frac{1}{2}+\frac{1}{5}+\frac{1}{8}-\frac{1}{4}+\frac{1}{9} \ldots \\
& =1-\frac{1}{2}+\frac{1}{3}-\frac{1}{4}+\frac{1}{6}-\ldots \ldots \ldots . . \\
& =\log 2 . \\
2 & =1 .
\end{aligned}
$$

Fourth Fallacy. This fallacy is very similar to that last given. We have

$$
\begin{aligned}
\log 2 & =1-\frac{1}{2}+\frac{1}{3}-\frac{1}{4}+\frac{1}{5}-\frac{1}{8}+\ldots \\
& =\left(1+\frac{1}{8}+\frac{1}{5}+\ldots\right)-\left(\frac{1}{2}+\frac{1}{4}+\frac{1}{6}+\ldots\right) \\
& =\left\{\left(1+\frac{1}{8}+\frac{1}{5}+\ldots\right)+\left(\frac{1}{2}+\frac{1}{4}+\frac{1}{6}+\ldots\right)\right\}-2\left(\frac{1}{2}+\frac{1}{4}+\frac{1}{8}+\ldots\right) \\
& =\left\{1+\frac{1}{2}+\frac{1}{3}+\ldots\right\}-\left(1+\frac{1}{2}+\frac{1}{3}+\ldots\right) \\
& =0 .
\end{aligned}
$$

Fifth Fallacy. We have

$$
\sqrt{a} \times \sqrt{b}=\sqrt{a \bar{b}} .
$$

Hence

$$
\sqrt{-1} \times \sqrt{-1}=\sqrt{(-1)(-1)} \text {, }
$$

therefore, $(\sqrt{-1})^{2}=\sqrt{1}$, that is, $-1=1$. 
Sixth Fallacy. The following demonstration depends on the fact that an algebraical identity is true whatever be the symbols used in it, and it will appeal only to those who are familiar with this fact. We have, as an identity,

$$
\sqrt{x-y}=i \sqrt{y-x}
$$

where $i$ stands either for $+\sqrt{-1}$ or for $-\sqrt{-1}$. Now an identity in $x$ and $y$ is necessarily true whatever numbers $x$ and $y$ may represent. First put $x=a$ and $y=b$,

$$
\therefore \sqrt{a-b}=i \sqrt{b-a}
$$

Next put $x=b$ and $y=a$,

$$
\therefore \sqrt{b-a}=i \sqrt{a-b}
$$

Also since (i) is an identity, it follows that in (ii) and (iii) the symbol $i$ must be the same, that is, it represents $+\sqrt{-1}$ or $-\sqrt{-1}$ in both cases. Hence, from (ii) and (iii), we have

$$
\begin{gathered}
\sqrt{a-b} \sqrt{b-a}=i^{2} \sqrt{b-a} \sqrt{a-b}, \\
\therefore 1=i^{2},
\end{gathered}
$$

that is,

$$
1=-1 \text {. }
$$

Seventh Fallacy. The following fallacy is due to D'Alembert*. We know that if the product of two numbers is equal to the product of two other numbers, the numbers will be in proportion, and from the definition of a proportion it follows that if the first term is greater than the second, then the third term will be greater than the fourth: thus, if $a d=b c$, then $a: b=c: d$, and if in this proportion $a>b$, then $c>d$. Now if we put $a=d=1$ and $b=c=-1$ we have four numbers which satisfy the relation $a d=b c$ and such that $a>b$; hence, by the proposition, $c>d$, that is, $-1>1$, which is absurd.

Eighth Fallacy. The mathematical theory of probability leads to various paradoxes: of these one specimen $\dagger$ will suffice. Suppose three coins to be thrown up and the fact whether each comes down head or tail to be noticed. The probability that all three coins come down head is clearly $(1 / 2)^{3}$, that is, is $1 / 8$;

- Opuscules Mathématiques, Paris, 1761, rol. 1, p. 201.

+ See Nature, Feb. 15, March 1, 1894, vol. xLIx, pp. 365-366, 413. 
similarly the probability that all three come down tail is $1 / 8$; hence the probability that all the coins come down alike (i.e. either all of them heads or all of them tails) is $1 / 4$. But, of three coins thus thrown up, at least two must come down alike: now the probability that the third coin comes down head is $1 / 2$ and the probability that it comes down tail is $1 / 2$, thus the probability that it comes down the same as the other two coins is $1 / 2$ : hence the probability that all the coins come down alike is $\mathbf{1 / 2}$. I leave to my readers to say whether either of these conflicting conclusions is right, and, if so, which is correct.

Arithmetical Problems. To the above examples I may add the following standard questions, or recreations.

The first of these questions is as follows. Two clerks, $\boldsymbol{A}$ and $B$, are engaged, $A$ at a salary commencing at the rate of (say) $£ 100$ a year with a rise of $£ 20$ every year, $B$ at a salary commencing at the same rate of $£ 100$ a year with a rise of $£ 5$ every half-year, in each case payments being made half-yearly; which has the larger income? The answer is $B$; for in the first year $A$ receives $£ 100$, but $B$ receives $£ 50$ and $£ 55$ as his two half-yearly payments and thus receives in all $£ 105$. In the second year $A$ receives $£ 120$, but $B$ receives $£ 60$ and $£ 65$ as his two half-yearly payments and thus receives in all $£ 125$. In fact $B$ will always receive $£ 5$ a year more than $A$.

Another simple arithmetical problem is as follows. A hymnboard in a church has four grooved rows on which the numbers of four hymns chosen for the service are placed. The hymnbook in use contains 700 hymns. What is the smallest number of plates, each carrying one digit, which must be kept in stock so that the numbers of any four different hymns selected can be displayed; and how will the result be affected if an inverted 6 can be used for a 9 ? The answers are 86 and 81 . What are the answers if a digit is painted on each side of each plate?

As another question take the following. A man bets $1 / n$th of his money on an even chance (say tossing heads or tails with a penny): he repeats this again and again, each time betting $1 / n$th of all the money then in his possession. If, finally, the number of times he has won is equal to the number 
of times he has lost, has he gained or lost by the transaction? He has, in fact, lost.

Here is another simple question to which not unfrequently I have received incorrect answers. One tumbler is half-full of wine, another is half-full of water: from the first tumbler a teaspoonful of wine is taken out and poured into the tumbler containing the water: a teaspoonful of the mixture 1 in the second tumbler is then transferred to the first tumbler. As the result of this double transaction, is the quantity of wine removed from the first tumbler greater or less than the quantity of water removed from the second tumbler? In my experience the majority of people will say it is greater, but this is not the case.

Here is another paradox dependent on the mathematical theory of probability. Suppose that a player at bridge or whist asserts that an ace is included among the thirteen cards dealt to him, and let $p$ be the probability that he has another ace among the other cards in his hand. Suppose, however, that he asserts that the ace of hearts is included in the thirteen cards dealt to him, then the probability, $q$, that he has another ace among the other cards in his hand is greater than was the probability $p$ in the first case. For, if $r$ is the probability that when he has one ace it is the ace of hearts, we have $p=r \cdot q$, and since $p, q, r$ are proper fractions, we must have $q$ greater than $p$, which at first sight appears to be absurd.

Permutation Problems. Many of the problems in permutations and combinations are of considerable interest. As a simple illustration of the very large number of ways in which cornbinations of even a few things can be arranged, I may note that there are 500,291833 different ways in which change for a sovereign can be given in current coins*, including therein the obsolescent double-florin, and crown; also that as many as 19,958400 distinct skeleton cubes can be formed with twelve differently coloured rods of equal length $\dagger$; while there are no less than $(52 !) /(13 !)^{4}$, that is, $53644,737765,488792,839237,44,0000$

- The Tribune, September 3, 1906.

+ Mathematical Tripos, Cambridge, Part I, 1894. 
possible different distributions of hands at bridge or whist with a pack of fifty-two cards.

Voting Problems. As a simple example on combinations I take the cumulative vote as affecting the representation of a minority. If there are $p$ electors each having $r$ votes of which not more than $s$ may be given to one candidate, and $n$ men are to be elected, then the least number of supporters who can secure the election of a candidate must exceed $p r /(n s+r)$.

The Knights of the Round Table. A far more difficult permutation problem consists in finding as many arrangements as possible of $n$ people in a ring so that no one has the same two neighbours more than once. It is a well-known proposition that $n$ persons can be arranged in a ring in $(n-1) ! / 2$ different ways. The number of these arrangements in which all the persons have different pairs of neighbours on each occasion cannot exceed $(n-1)(n-2) / 2$, since this gives the number of ways in which any assigned person may sit between every possible pair selected from the rest. But in fact it is always possible to determine $(n-1)(n-2) / 2$ arrangements in which no one has the same two neighbours on any two occasions.

Solutions for various values of $n$ have been given. Here for instance (if $n=8$ ) are 21 arrangements * of eight persons. Each arrangement may be placed round a circle, and no one has the same two neighbours on any two occasions.

$$
\begin{aligned}
& \text { 1.2.3.4.5.6.7.8; 1.2.5.6.8.7.4.3; 1.2.7.8.4.3.5.6; } \\
& \text { 1.3.5.2.7.4.8.6; 1.3.7.4.6.8.2.5; 1.3.8.6.2.5.7.4; } \\
& \text { 1.4.2.6.3.8.5.7; 1.4.3.8.7.5.6.2; 1.4.5.7.6.2.3.8; } \\
& \text { 1.5.6.4.3.7.8.2; 1.5.7.3.8.2.6.4; 1.5.8.2.4.6.3.7; } \\
& \text { 1.6.2.7.5.3.8.4; 1.6.3.5.8.4.2.7; 1.6.4.8.2.7.3.5; } \\
& \text { 1.7.4.2.5.8.6.3; 1.7.6.3.2.4.5.8; 1.7.8.5.6.3.4.2; } \\
& \text { 1.8.2.3.7.6.4.5; 1.8.4.5.3.2.7.6; 1.8.6.7.4.5.2.3. }
\end{aligned}
$$

The methods of determining these arrangements are lengthy, and far from easy.

- Communicated to me by Mr E. G. B. Bergholt, May, 1906, see The Secretary and The Queen, August, 1906. Mr Dudeney had given the problem for the case when $n=6$ in 1905 , and informs me that the problem has been solved by Mr E. D. Bewley when $n$ is even, and that he has a general method applicable when $n$ is odd. Various memoirs on the subject have appeared in the mathematical journals.

B. $\mathbf{R}$. 
The Ménage Problem*. Another difficult permutation problem is concerned with the number $x$ of possible arrangements of $n$ married couples, seated alternately man and woman, round a table, the $n$ wives being in assigned positions, and the $n$ husbands so placed that a man does not sit next to his wife.

The solution involves the theory of discordant permutations $\dagger$, and is far from easy. I content myself with noting the results when $n$ does not exceed 10. When $n=3, x=1$; when $n=4$, $x=2$; when $n=5, x=13$; when $n=6, x=80$; when $n=7$, $x=579$; when $n=8, x=4738$; when $n=9, x=43387$; and when $n=10, x=439792$.

Bachet's Weights Problem $\nmid$. It will be noticed that a considerable number of the easier problems given in the last chapter either are due to Bachet or were collected by him in his classical Problèmes. Among the more difficult problems proposed by him was the determination of the least number of weights which would serve to weigh any integral number of pounds from $1 \mathrm{lb}$. to $40 \mathrm{lbs}$. inclusive. Bachet gave two solutions: namely, (i) the series of weights of $1,2,4,8,16$, and $32 \mathrm{lbs}$; (ii) the series of weights of $1,3,9$, and $27 \mathrm{lbs}$.

If the weights may be placed in only one of the scale-pans, the first series gives a solution, as had been pointed out in 1556 by Tartaglia $\S$.

Bachet, however, assumed that any weight might be placed in either of the scale-pans. In this case the second series gives the least possible number of weights required. His reasoning is as follows. To weigh $1 \mathrm{lb}$. we must have a $1 \mathrm{lb}$. weight. To weigh 2 lbs. we must have in addition either a $2 \mathrm{lb}$. weight or a 3 lb. weight; but, whereas with a $2 \mathrm{lb}$. weight we can weigh $1 \mathrm{lb}, 2 \mathrm{lbs}$, and $3 \mathrm{lbs}$, with a $3 \mathrm{lb}$. weight we can weigh $1 \mathrm{lb} .,(3-1) \mathrm{lbs} ., 3 \mathrm{lbs}$, and $(3+1)$ lbs. Another weight of $9 \mathrm{lbs}$. will enable us to weigh all weights from $1 \mathrm{lb}$. to $13 \mathrm{lbs}$; and we get thus a greater range than is obtainable with any

* Thêorie des Nombres, by E. Lucas, Paris, 1891, pp. 215, 491-495.

† See P. A. MacMahon, Combinatory Analysis, vol. I, Cambridge, 1915, pp. $253-256$.

† Bachet, Appendix, problem v, p. 215.

$\$$ Trattato de' numeri e misure, Venice, 1556, vol. II, bk, I, chap. xVI, art. 32. 
weight less than $9 \mathrm{lbs}$. Similarly weights of $1,3,9$, and $27 \mathrm{lbs}$. suffice for all weights up to $40 \mathrm{lbs}$, and weights of $1,3,3^{2}, \ldots, 3^{n-1} \mathrm{lbs}$. enable us to weigh any integral number of pounds from $1 \mathrm{lb}$. to $\left(1+3+3^{2}+\ldots 3^{n-1}\right)$ lbs., that is, to $\frac{1}{2}\left(3^{n}-1\right) \mathrm{lbs}$.

To determine the arrangement of the weights to weigh any given mass we have only to express the number of pounds in it as a number in the ternary scale of notation, except that in finding the successive digits we must make every remainder either 0,1 , or -1 : to effect this a remainder 2 must be written as $3-1$, that is, the quotient nust be increased by unity, in which case the remainder is -1 . This is explained in most text-books on algebra.

Bachet's argument does not prove that his result is unique or that it gives the least possible number of weights required. These omissions have been supplied by Major MacMahon, who has discussed the far more difficult problem (of which Bachet's is a particular case) of the determination of all possible sets of weights, not necessarily unequal, which enable us to weigh any integral number of pounds from 1 to $n$ inclusive, (i) when the weights may be placed in only one scale-pan, and (ii) when any weight may be placed in either scale-pan. He has investigated also the modifications of the results which are necessary when we impose either or both of the further conditions (a) that no other weighings are to be possible, and (b) that each weighing is to be possible in only one way, that is, is to be unique*.

The method for case (i) consists in resulving $1+x+x^{2}+\ldots+x^{n}$ into factors, each factor being of the form $1+x^{a}+x^{2 a}+\ldots+x^{m a}$; the number of solutions depends on the composite character of $n+1$. The method for case (ii) consists in resolving the expression $x^{-n}+x^{-n+1}+\ldots+x^{-1}+1+x+\ldots+x^{n-1}+x^{n}$ into factors, each factor being of the form $x^{-m a}+\ldots+x^{-a}+1+x^{a}+\ldots+x^{m a}$; the number of solutions depends on the composite character of $2 n+1$.

- See his article in the Quarterly Journal of Mathematics, 1886, 701. IxI, pp. 367-373. An account of the method is given in Nature, Dec. 4, 1890, vol. xLII, pp. 113-114. 
Bachet's problem falls under case (ii), $n=40$. MacMahon's analysis shows that there are eight such ways of factorizing $x^{-40}+x^{-39}+\ldots+1+\ldots+x^{39}+x^{40}$. First, there is the expression itself in which $a=1, m=40$. Second, the expression is equal to $\left(1-x^{81}\right) / x^{40}(1-x)$, which can be resolved into the product of $\left(1-x^{3}\right) / x(1-x)$ and $\left(1-x^{81}\right) / x^{39}\left(1-x^{3}\right)$; hence it can be resolved into two factors of the form given above, in one of which $a=1, m=1$, and in the other $a=3, m=13$. Third, similarly, it can be resolved into two such factors, in one of which $a=1, m=4$, and in the other $a=9, m=4$. Fourth, it can be resolved into three such factors, in one of which $a=1, m=1$, in another $a=3, m=1$, and in the other $a=9, m=4$. Fifth, it can be resolved into two such factors, in one of which $a=1, m=13$, and in the other $a=27, m=1$. Sixth, it can be resolved into three such factors, in one of which $a=1, m=1$, in another $a=3, m=4$, and in the other $a=27, m=1$. Seventh, it can be resolved into three such factors, in one of which $a=1, m=4$, in another $a=9, m=1$, and in the other $a=27, m=1$. Eighth, it can be resolved into four such factors, in one of which $a=1, m=1$, in another $a=3$, $m=1$, in another $a=9, m=1$, and in the other $a=27, m=1$.

These results show that there are eight possible sets of weights with which any integral number of pounds from 1 to 40 can be weighed subject to the conditions (ii), (a), and (b). If we denote $p$ weights each equal to $w$ by $w^{p}$, these eight solutions are $11^{40} ; 1,3^{13} ; 1^{4}, 9^{4} ; 1,3,9^{4} ; 1^{13}, 27 ; 1,3^{4}, 27$; $1^{4}, 9,27 ; 1,3,9,27$. The last of these is Bachet's solution: not only is it that in which the least number of weights are employed, but it is also the only one in which all the weights are unequal.

Problems in Higher Arithmetic. Many mathematicians take a special interest in the theorems of higher arithmetic: such, for example, as that every prime of the form $4 n+1$ and every power of it is expressible as the sum of two squares*, and that the first and second powers can be expressed thus in only one way. For instance, $13=3^{2}+2^{2}, 13^{2}=12^{2}+5^{2}, 13^{3}=46^{2}+9^{2}$,

- Fermat's Diophantus, Toulouse, 1670, bk. IIr, prop. 22, p. 127; or Brassinne's Précis, Paris, 1853, p. 65. 
and so on. Similarly $41=5^{2}+4^{2}, 41^{2}=40^{2}+9^{2}, 41^{3}=236^{2}+115^{2}$, and so on. Propositions such as the one just quoted may be found in text-books on the theory of numbers and therefore lie outside the limits of this work, but there are one or two questions in higher arithmetic involving points not yet quite cleared up which may find a place here. I content myself with the facts and shall not give the mathematical unalysis.

PRIMes. The first of these is concerned with the possibility of determining readily whether a given number is prime or not. No test applicable to all numbers is known, though of course we can get tests for numbers of certain forms. It is difficult to believe that a problem which has completely baffled all modern mathematicians could have been solved in the seventeenth century, but it is interesting to note that in 1643, in answer to a question in a letter whether the number 100895,598169 was a prime, Fermat replied at once that it was the product of 898423 and 112303, both of which were primes. How many mathematicians to-day could answer such a question with ease?

Mersenne's Numbers. A curious assertion (in this case only partially correct) about the prime or composite character of numbers of the form $2^{p}-1$ (which we may denote by $N$ ) is to be found in Mersenne's Cogitata Physico-Mathematica, published in 1644 . In the preface to that work a statement is made about perfect numbers, which implies that the only values of $p$ not greater than 257 which make $N$ prime are $1,2,3,5,7,13$, $17,19,31,67,127$, and 257. Some years ago I gave reasons for thinking that 67 was a misprint for 61 . Until 1911, no error in this corrected statement was established, and it was gradually verified for all except sixteen values of $p$. In 1911, however, it was proved, that $N$ was prime when $p=89$, and three years later that it was prime when $p=107$ : two facts at variance with Mersenne's statement. The prime or composite character of $N$ now remains unknown for only ten values of $p$, namely 139, 149, $157,167,193,199,227,229,241$, and 257.

We may safely say that the methods used to-day in estab- 
lishing the known results for many of the higher values of $p$ could not have been employed by Mersenne. It would be interesting to discover how he reached his conclusions, which are true if $p$ does not exceed 88. Some recent writers conjecture that his statement was the result of a guess, intelligent though erroneous, as to the possible forms of $p$ : I find it difficult to accept this opinion, but further discussion of the problem would be out of place here*.

Perfect Numbers $†$. The theory of perfect numbers depends directly on that of Mersenne's Numbers. A number is said to be perfect if it is equal to the sum of all its integral subdivisors. Thus the subdivisors of 6 are 1,2 , and 3 ; the sum of these is equal to 6 ; hence 6 is a perfect number.

It is probable that all perfect numbers are included in the formula $2^{p-1}\left(2^{p}-1\right)$, where $2^{p}-1$ is a prime. Euclid proved that any number of this form is perfect; Euler showed that the formula includes all even perfect numbers; and there is reason to believe-though a rigid demonstration is wantingthat an odd number cannot be perfect, at any rate no odd number less than 2,000,000 can be perfect. If we assume that there are no odd perfect numbers, then every perfect number is of the above form. It is easy to establish that every number included in this formula (except when $p=2$ ) is congruent to unity to the modulus 9 , that is, when divided by 9 leaves a remainder 1 ; also that either the last digit is a 6 or the last two digits are 28.

Thus, if $p=2,3,5,7,13,17,19,31,61$, then by Mersenne's rule the corresponding values of $2^{p}-1$ are prime; they are $3,7,31$, $127,8191,131071,524287,214748364 i$, 2305843009213693951;

* In recent editions of this book I deroted a chapter to this problem and ita history, but having regard to the fact that it is now known that Mersenne's statement is not true, the above notice suffices. References to memoirs on the subject are given by L. E. Dickson, History of the Theory of Numbers, vol. I, Washington, 1919.

$\dagger$ On the theory of perfect numbers, see bibliographical references by H. Brocard, L'Intermédiaire des Mathématiciens, Paris, 1895, vol. Ix, pp. 52-54; and 1905, vol. xII, p. 19. The first volume of the second edition of the Frenoh translation of this book contains (pp. 280-294) a summary of the leading investigation on Perfect Numbers, as also some remarks on Amioable Numbers. 
and the corresponding perfect numbers are $6,28,496,8128$, $33550336,8589869056,137438691328,2305843008139952128$, and 2658455991569831744654692615953842176.

Euler's Biquadrate THeorem*. Another theorem, believed to be true but as yet unproved, is that the arithmetical sum of the fourth powers of three numbers cannot be the fourth power of a number; in other words, we cannot find values of $x, y, z, v$, which satisfy the equation $x^{4}+y^{4}+z^{4}=v^{4}$. The proposition is not true of an algebraical-sum, for Euler gave more than one solution of the equation $x^{4}+y^{4}=z^{4}+v^{4}$, for instance, $x=542, y=103, z=359, v=514$.

GOLDBACH'S THEOREM. Another interesting question is whether all even integers can be expressed as a sum of two primes. Asymptotic expressions for the number of ways of expressing even numbers as the sum of two primes have been suggested and are consistent with numerical tests. This suggests that every even number above some limit is expressible in this way; and if so, unless this limit is less than 5000, up to which number the theorem has been empirically established $\dagger$, it may be accepted as true.

LAGRANGE's Theorem+. Another theorem in higher arithmetic which, as far as I know, is still unsolved, is to the effect that every prime of the form $4 n-1$ is the sum of a prime of the form $4 n+1$ and of double another prime also of the form $4 n+1$; for example, $23=13+2 \times 5$. Lagrange, however, added that it was only by induction that he arrived at the result.

Fermat's Theorem on Binary Powers. Fermat enriched mathematics with a multitude of new propositions. With one exception all these have been proved or are believed to be true. This exception is his theorem on binary powers, in which

* See Euler, Commentationes Arithmeticae Collectae, Petrograd, 1849, vol. I. pp. $473-476$; vol. II, pp. $450-456$. A somewhat similar unproved theorem is that the sum of $n$ numbers of the form $x^{k}$ cannot be of the form $u^{k}$ if $n$ is less than $k$.

† Transactions of the Halle Academy (Naturforschung), vol. Lxxir, Halle, 1897, pp. 5-214: see also L'Intermédiaire des Mathématiciens, 1903, vol. x, and 1904, vol. $x r$.

‡Nouveaux Mémoires de l'Academie Royale des Sciences, Berlin, 1775, p. 356. 
he asserted that all numbers of the form $2^{m}+1$, where $m=2^{n}$, are primes*, but he added that, though he was convinced of the truth of this proposition, he could not obtain a valid demonstration.

It may be shown that $2^{m}+1$ is composite if $m$ is not a power of 2 , but of course it does not follow that $2^{m}+1$ is a prime if $m$ is a power of 2 , say, $2^{n}$. As a matter of fact the theorem is not true. In 1732 Euler $\dagger$ showed that if $n=5$ the formula gives 4294,967297 , which is equal to $641 \times 6,700417$ : curiously enough, these factors can be deduced at once from Fermat's remark on the possible factors of numbers of the form $2^{m} \pm 1$, from which it may be shown that the prime factors (if any) of $2^{32}+1$ must be primes of the form $64 n+1$.

During the last thirty years it has been shown+ that the resulting numbers are composite when $n=6,7,8,9,11,12,18$, 23, 36, 38 and 73. The digits in the last of these numbers are so numerous that, if the number were printed in full with the type and number of pages used in this book, many more volumes would be required than are contained in all the public libraries in the world. I believe that Eisenstein asserted that the number of primes of the form $2^{m}+1$, where $m=2^{n}$, is infinite: the proof has not been published, but perhaps it might throw some light on the general theory.

Fermat's Last Theorem. I pass now to another assertion made by Fermat which hitherto has not been proved. This, which is sometimes known as Fermat's Last Theorem, is to the effect $\S$ that no integral values of $x, y, z$ can be found to satisfy

* Letter of Oct. 18, 1640, Opera, Toulouse, 1679, p. 162: or Brassinne's Précis, p. 143.

†Commentarii Academiae Scientiarum Petropolitanae, Petrograd, 1738, vol. vI, p. 104; see also Novi Comm. Acad. Sci. Petrop., Petrograd, 1764, vol. Ix, p. 101: or Commentationes Arithmeticae Collectae, Petrograd, 1849, vol. r, pp. 2, 357 .

¥ For the factors and bibliographical references, see A. J. C. Cunningham and A. E. Western, Transactions of the London Mathematical Society, 1903, series 2, vol. I, p. 175; and J. C. Morehead and A. E. Western, Bulletin of the American Mathematical Society, 1909, vol, xvI, pp. I-6.

\$ Fermat's enunciation will be found in his edition of Diophantus, Toulouse, 1670, bls. II, qu. 8, p. 61; or Brassinne's Précis, Paris, 1853, p. 53. For 
the equation $x^{n}+y^{n}=z^{n}$, if $n$ is an integer greater than 2 . This proposition has acquired extraordinary celebrity from the fact that no general demonstration of it has been given, but there is no reason to doubt that it is true.

Fermat seems to have discovered its truth first* for the case $n=3$, and then for the case $n=4$. His proof for the former of these cases is lost, but that for the latter is extant $\dagger$, and a similar proof for the case of $n=3$ was outlined by Eulert. These proofs depend upon showing that, if three integral values of $x, y, z$ can be found which satisfy the equation, then it will be possible to find three other and smaller integers which also satisfy it: in this way finally we show that the equation must be satisfied by three values which obviously do not satisfy it. Thus no integral solution is possible. This method is inapplicable to the general case.

Fermat's discovery of the general theorem was made later. A proof can be given on the assumption that every number can be resolved into the product of powers of primes in only one way. The assumption is true of real integers, but is not necessarily true for algebraic integers-an algebraic integer being defined as a root of an equation

$$
x^{n}+\alpha_{1} x^{n-1}+\ldots+\alpha_{n}=0,
$$

whose cuefficients, $\alpha$, are arithmetical integers; for instance, $a+b \sqrt{-m}$, where $a, b$, and $m$, are arithmetical integers, is an algebraic integer. Thus, admitting the use of these generalized integers, 21 can be expressed in three ways as the product of primes, namely, 3 and 7 , or of $4+\sqrt{-5}$ and $4-\sqrt{-5}$, or of $1+2 \sqrt{-5}$ and $1-2 \sqrt{-5}$; and similarly, there are values of $n$ for which Fermat's equation leads to expressions which can be factorized in more than one way. It is possible that Fermat's argument rested on the above erroneous supposition, but this is an unsupported conjecture. At any rate he asserted bibliographical references, see L. . . Dickson, History of the Theory of Numbers, Washington, 1920, vol. II, ch. 26; see also L. J. Mordell, Fermat's Last Theorem, Cambridge, 1921.

* See a Letter from Fermat quoted in my History of Mathematics, London, chapter $\mathrm{xv}$.

$\dagger$ Fermat's Diophantus, note on p. 339; or Brassinne's Précis, p. 127.

\# Euler's Algebra (English trans. 1797), vol. II, chap. Xv, p. 247 : one point was overlooked by Euler, but the omission can be supplied. 
definitely that he had a valid proof-demonstratio mirabilis sane-and the fact that no theorem on the subject which he stated he had proved has been subsequently shown to be false must weigh strongly in his favour; the more so because in making the one incorrect statement in his writings (namely, that about binary powers) he added that he could not obtain a satisfactory demonstration of it.

It must be remembered that Fermat was a mathematician of quite the first rank who had made a special study of the theory of numbers. The subject is in itself one of peculiar interest and elegance, but its conclusions have little practical importance, and for long it was studied by only a few mathematicians. This is the explanation of the fact that it took more than a century before some of the simpler results which Fermat had enunciated were proved, and thus it is not surprising that a proof of the theorem which he succeeded in establishing only towards the close of his life should involve great difficulties.

In 1823 Legendre* obtained a proof for the case of $n=5$; in 1832 Lejeune Dirichlet $\nmid$ gave one for $n=14$; and in 1840 Lamé and Lebesgue + gave proofs for $n=7$.

The proposition appears to be true, and in 1849 Kummer§, by means of ideal primes, proved it to be so for all numbers which satisfy certain Bernoullian conditions. The only numbers less than 100 which do not do so are $37,59,67$, and the theorem can, by other arguments, be proved for these three cases. Other tests have been established; for instance, $A$. Wieferich\| has shown that if the equation is soluble in integers prime to $n$, where $n$ is an odd prime, then $2^{n-1}-1$ is divisible by $n^{2}$. No exception to the theorem has yet been found, and by one means or another, the number of cases which require special discussion has been reduced to but few. I may add that to prove the truth of the proposition when $n$ is greater than 4, obviously it

* Reprinted in his Thêorie des Nombres, Paris, 1830, vol. II, pp. 361-368: see also pp. $5,6$.

$\dagger$ Crelle's Journal, 1832, vol. Ix, pp. 390-393.

¥ Liouville's Journal, 1841, vol, v, pp. 195-215, 276-279, 348-349.

\$ References to Kummer's Memoirs are given in Smith's Report to the British Association on the Theory of Numbers, London, 1860.

\|l Crelle's Journal, Berlin 1909, vol. cxxxvi, pp. 293-302. 
is sufficient to confine ourselves to cases where $n$ is a prime. A prize* of 100,000 marks has been offered for a general proof, to be given before 2007 .

Naturally there has been much speculation as to how Fermat arrived at the result. The modern treatment of higher arithmetic is founded on the special notation and processes introduced by Gauss, who pointed out that the theory of discrete magnitude is essentially different from that of continuous magnitude, but until the end of the last century the theory of numbers was treated as a branch of algebra, and such proofs by Fermat as are extant involve nothing more than elementary geometry and algebra, and indeed some of his arguments do not involve any symbols. This has led some writers to think that Fermat used none but elementary algebraic methods. This may be so, but the following remark, which I believe is not generally known, rather points to the opposite conclusion. He had proposed, as a problem to the English mathematicians, to show that there was only one integral solution of the equation $x^{2}+2=y^{3}$ : the solution evidently being $x=5, y=3$. On this he has a note $f$ to the effect that there was no difficulty in finding a solution in rational fractions, but that he had discovered an entirely new method-sane pulcherrima et subtilissima-which enabled him to solve such questions in integers. It was his intention to write a work $\ddagger$ on his researches in the theory of numbers, but it was never completed, and we know but little of his methods of analysis. I venture however to add my private suspicion that continued fractions played a not unimportant part in his researches, and as strengthening this conjecture I may note that some of his more recondite results-such as the theorem that a prime of the form $4 n+1$ is expressible as the sum of two squares-may be established with comparative ease by properties of such fractions.

* L'Intermediaire des Muthématiciens, vol. $\mathbf{x \nabla}$, pp. 217-218, for references and details.

† Fermat's Diophantus, bk. vi, prop. 19, p. 320; or Brassinne's Précis, p. 122.

† Fermat's Diophantus, bk. IV, prop. 31, p. 181 ; or Brassinne's Précis, p. 82. 
CHAPTER III.

GEOMETRICAL RECREATIONS.

In this chapter and the next one I propose to enumerate certain geometrical questions, puzzles, and games, the discussion of which will not involve necessarily any considerable use of algebra or arithmetic. Most of this chapter is devoted to questions which are of the nature of formal propositions: the next chapter contains a description of various amusements.

In accordance with the rule I laid down for myself in the preface, I exclude the detailed discussion of theorems which involve advanced mathematics. Moreover (with one or two exceptions) I exclude any mention of the numerous geometrical paradoxes which depend merely on the inability of the eye to compare correctly the dimensions of figures when their relative position is changed. This apparent deception does not involve the conscious reasoning powers, but rests on the inaccurate interpretation by the mind of the sensations derived through the eyes, and I do not consider such paradoxes as coming within the domain of mathematics.

Geometrical Fallacies. Most educated Englishmen are acquainted with the series of logical propositions in geometry associated with the name of Euclid, but it is not known so generally that these propositions were supplemented originally by certain exercises. Of such exercises Euclid issued three 
geries: two containing easy theorems or problems, and the third consisting of geometrical fallacies, the exrors in which the student was required to find.

The collection of fallacies prepared by Euclid is lost, and tradition has not preserved any record as to the nature of the erroneous reasoning or conclusions; but, as an illustration of such questions, I append a few demonstrations, leading to obviously impossible results. Perhaps they may amuse any one to whom they are new. I leave the discovery of the errors to the ingenuity of my readers.

First Fallacy*. To prove that a right angle is equal to an angle which is greater than a right angle. Let $A B C D$ be a rectangle. From $A$ draw a line $A E$ outside the rectangle, equal to $A B$ or $D C$ and making an acute angle with $A B$, as

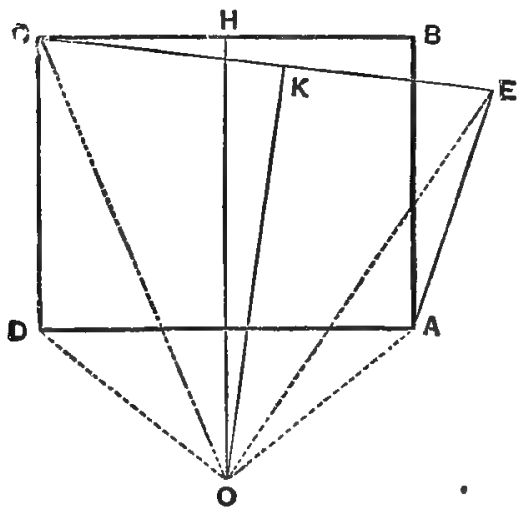

indicated in the diagram. Bisect $C B$ in $H$, and through $H$ draw $H O$ at right angles to $C B$. Bisect $C E$ in $K$, and through $K$ draw $K O$ at right angles to $C E$. Since $C B$ and $C E$ are not parallel the lines $H O$ and $K O$ will meet (say) at $O$. Join $O A$, $O E, O C$, and $O D$.

The triangles $O D C$ and $O A E$ are equal in all respects. For, since $K O$ bisects $C E$ and is perpendicular to it, we have

- I believe that this and the fourth of these fallacies were first published in this book. They particularly interested Mr C. L. Dodgson; see the Lewis Carroll Picture Book, London, 1899. pp. 264, 266, where they appear in the form in which I originalls gave them. 
$O C=O E$. Similarly, since $H O$ bisects $C B$ and $D A$ and is perpendicular to them, we have $O D=O A$. Also, by construction, $D C=A E$. Therefore the three sides of the triangle $O D C$ are equal respectively to the three sides of the triangle $O A E$. Hence, by Euc. I. 8, the triangles are equal; and therefore the angle $O D C$ is equal to the angle $O A E$.

Again, since $H O$ bisects $D A$ and is perpendicular to it, we have the angle $O D A$ equal to the angle $O A D$.

Hence the angle $A D C$ (which is the difference of $O D C$ and $O D A$ ) is equal to the angle $D A E$ (which is the difference of $O A E$ and $O A D$ ). But $A D C$ is a right angle, and $D A E$ is necessarily greater than a right angle. Thus the result is impossible.

Second Fallacy*. To prove that a part of a line is equal to the whole line. Let $A B C$ be a triangle; and, to fix our ideas, let us suppose that the triangle is scalene, that the angle $B$ is

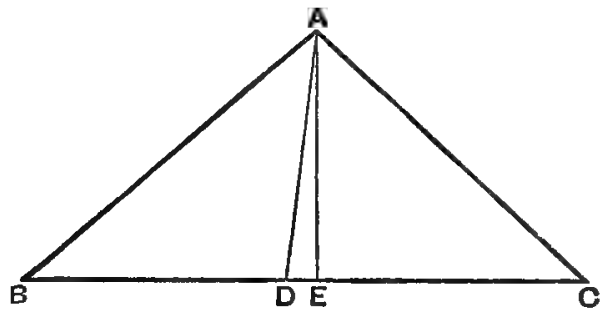

acute, and that the angle $A$ is greater than the angle $C$. From $A$ draw $A D$ making the angle $B A D$ equal to the angle $C$, and cutting $B C$ in $D$. From $A$ draw $A E$ perpendicular to $B C$.

The triangles $A B C, A B D$ are equiangular; hence, by Euc. vI. 19, $\triangle A B C: \triangle A B D=A C^{2}: A D^{2}$.

Also the triangles $A B C, A B D$ are of equal altitude; hence, by Euc. vI. 1,

$$
\begin{aligned}
\triangle A B C: \triangle A B D & =B C: B D, \\
\therefore A C^{2}: A D^{2} & =B C: B D . \\
\therefore \frac{A C^{2}}{B C} & =\frac{A D^{2}}{B D} .
\end{aligned}
$$

- See a note by M. Coccoz in L'Illustration, Paris, Jan. 12, 1895. 
Hence, by Euc. Ir. 13,

$$
\begin{aligned}
\frac{A B^{2}+B C^{2}-2 B C \cdot B E}{B C} & =\frac{A B^{2}+B D^{2}-2 B D \cdot B E}{B D} \\
\therefore \frac{A B^{2}}{B C}+B C-2 B E & =\frac{A B^{2}}{B D}+B D-2 B E \\
\therefore \frac{A B^{2}}{B C}-B D & =\frac{A B^{2}}{B D}-B C . \\
\therefore \frac{A B^{2}-B C \cdot B D}{B C} & =\frac{A B^{2}-B C \cdot B D}{B D} . \\
\therefore B C & =B D,
\end{aligned}
$$

a result which is impossible.

Third Fallacy*. To prove that the sum of the lengths of two sides of any triangle is equal to the length of the third side.

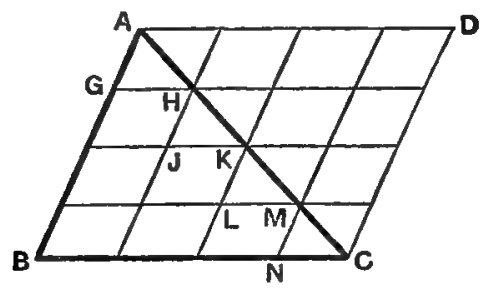

Let $A B C$ be a triangle. Complete the parallelogram of which $A B$ and $B C$ are sides. Divide $A B$ into $n+1$ equal parts, and through the points so determined draw $n$ lines parallel to $B C$. Similarly, divide $B C$ into $n+1$ equal parts, and through the points so determined draw $n$ lines parallel to $A B$. The parallelogram $A B C D$ is thus divided into $(n+1)^{2}$ equal and similar parallelograms.

I draw the figure for the case in which $n$ is equal to 3, then, taking the parallelograms of which $A C$ is a diagonal, as indicated in the diagram, we have

$$
\begin{aligned}
A B+B C & =A G+H J+K L+M N \\
& +G H+J K+L M+N C .
\end{aligned}
$$

A similar relation is true however large $n$ may be. Now let $n$ increase indefinitely. Then the lines $A G, G H, \&$ c. will

- The Canterbury Puzzles, by H. E. Dodeney, London, 1907, pp. 26-28. 
get smaller and smaller. Finally the points $G, J, L, \ldots$ will approach indefinitely near the line $A C$, and ultimately will lie on it; when this is the case the sum of $A G$ and $G H$ will be equal to $A H$, and similarly for the other similar pairs of lines. Thus, ultimately,

$$
\begin{aligned}
A B+B C & =A H+H K+K M+M C \\
& =A C,
\end{aligned}
$$

a result which is impossible.

Fourth Fallacy. To prove that every triangle is isosceles. Let $A B C$ be any triangle. Bisect $B C$ in $D$, and through $D$ draw $D O$ perpendicular to $B C$. Bisect the angle $B A C$ by $A O$.

First. If $D O$ and $A O$ do not meet, then they are parallel. Therefore $A O$ is at right angles to $B C$. Therefore $A B=A C$.

Second. If $D O$ and $A O$ meet, let them meet in $O$. Draw $O E$ perpendicular to $A C$. Draw $O F$ perpendicular to $A B$. Join $O B, O C$.

Let us begin by taking the case where $O$ is inside the triangle, in which case $E$ falls on $A C$ and $F$ on BC.

The triangles $A O F$ and $A O E$ are

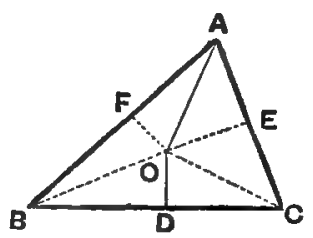
equal, since the side $A O$ is common, angle $O A F=$ angle $O A E$, and angle $O F A=$ angle $O E A$. Hence $A F=A E$. Also, the triangles $B O F$ and $C O E$ are equal. For since $O D$ bisects $B C$ at right angles, we have $O B=O C$; also, since the triangles $A O F$ and $A O E$ are equal, we have $O F=O E$; lastly, the angles at $F$ and $E$ are right angles. Therefore, by Euc. I. 47 and I. 8, the triangles $B O F$ and $C O E$ are equal. Hence $F B=E C$.

Therefore $A F+F B=A E+E C$, that is, $A B=A C$.

The same demonstration will cover the case where $D O$ and $A O$ meet at $D$, as also the case where they meet outside $B C$ but so near it that $E$ and $F$ fall on $A C$ and $A B$ and not on $A C$ and $A B$ produced.

Next take the case where $D O$ and $A O$ meet outside the triangle, and $E$ and $F$ fall on $A C$ and $A B$ produced. Draw 
$O E$ perpendicular to $A C$ produced. Draw $O F$ perpendicular to $A B$ produced. Join $O B, O C$.

Following the same argument as before, from the equality of the triangles $A O F$ and $A O E$, we obtain $A F=A E$; and, from the equality of the triangles $B O F$ and $C O E$, we obtain $F B=E C$. Therefore $A F-F B=A E-E C$, that is, $A B=A C$.

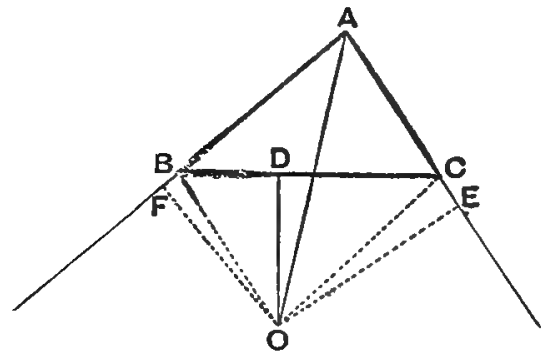

Thus in all cases, whether or not $D O$ and $A O$ meet, and whether they meet inside or outside the triangle, we have $A B=A C$ : and therefore every triangle is isosceles, a result which is impossible.

Fifth Fallacy*. To prove that $\pi / 4$ is equal to $\pi / 3$. On the hypothenuse, $B C$, of an isosceles right-angled triangle, $D B C$, describe an equilateral triangle $A B C$, the vertex $A$ being on the same side of the base as $D$ is. On $C A$ take a point $H$ so that $C H=C D$. Bisect $B D$ in $K$. Join $H K$ and let it cut $C B$ (produced) in $L$. Join $D L$. Bisect $D L$ at $M$, and through $M$ draw $M O$ perpendicular to $D L$. Bisect $H L$ at $N$, and through $N$ draw $N O$ perpendicular to $H L$. Since $D L$ and $H L$ intersect, therefore $M O$ and $N O$ will also intersect; moreover, since $B D C$ is a right angle, $M O$ and $N O$ both slope away from $D C$ and therefore they will meet on the side of $D L$ remote from $A$. Join $O C, O D, O H, O L$.

The triangles $O M D$ and $O M L$ are equal, hence $O D=O L$. Similarly the triangles $O N L$ and $O N H$ are equal, hence $O L=O H$. Therefore $O D=O H$. Now in the triangles $O C D$ and $O C H$, we have $O D=O H, C D=C H$ (by construction), and

- This ingenious fallacy is due to Captrin Turton: it appeared for the first time in the third edition of this work.

B. $\mathbb{R}$ 
$O C$ common, hence (by Euc. r. 8) the angle $O C D$ is equal to the angle $O C H$. Hence the angle $B C D$ is equal to the angle $B C H$, that is, $\pi / 4$ is equal to $\pi / 3$, which is absurd.

Sixth Fallacy*. To prove that, if two opposite sides of a quadrilateral are equal, the other two sides must be parallel. Let $A B C D$ be a quadrilateral such that $A B$ is equal to $D C$. Bisect $A D$ in $M$, and through $M$ draw $M O$ at right angles to $A D$. Bisect $B C$ in $N$, and draw $N O$ at right angles to $B C$.

If $M O$ and $N O$ are parallel, then $A D$ and $B C$ (which are at right angles to them) are also parallel.

If $M O$ and $N O$ are not parallel, let them meet in $O$; then $O$ must be either inside the quadrilateral as in the left-hand
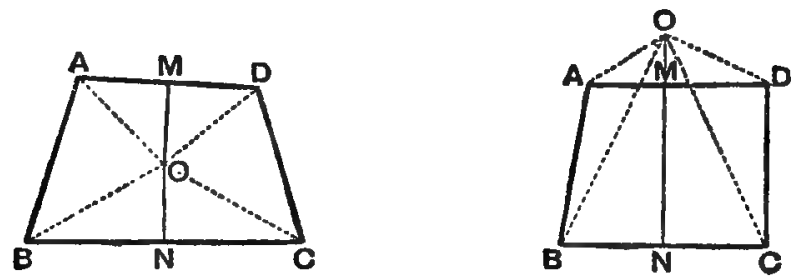

diagram or outside the quadrilateral as in the right-hand diagram. Join $O A, O B, O C, O D$.

Since $O M$ bisects $A D$ and is perpendicular to it, we have $O A=O D$, and the angle $O A M$ equal to the angle $O D M$. Similarly $O B=O C$, and the angle $O B N$ is equal to the angle $O C N$. Also by hypothesis $A B=D C$, hence, by Euc. I. 8, the triangles $O A B$ and $O D C$ are equal in all respects, and therefore the angle $A O B$ is equal to the angle $D O C$.

Hence in the left-hand diagram the sum of the angles $A O M, A O B$ is equal to the sum of the angles $D O M, D O C$; and in the right-hand diagram the difference of the angles $A O M, A O B$ is equal to the difference of the angles $D O M, D O C$; and therefore in both cases the angle $M O B$ is equal to the angle $M O C$, i.e. $O M$ (or $O M$ produced) bisects the angle $B O C$. But the angle $N O B$ is equal to the angle $N O C$, i.e. $O N$ bisects the angle $B O C$; hence $O M$ and $O N$ coincide in direction.

- Mathesis, October, 1893, series 2, vol. III, p. 224. 
Therefore $A D$ and $B C$, which are perpendicular to this direction, must be parallel. This result is not universally true, and the above demonstration contains a flaw.

Seventh Fallacy. The following argument is taken from a text-book on electricity, published in 1889 by two distinguished mathematicians, in which it was presented as valid. A given vector $O P$ of length $l$ can be resolved in an infinite number of ways into two vectors $O M, M P$, of lengths $l^{\prime}, l^{\prime \prime}$, and we can make $l^{\prime} / l^{\prime \prime}$ have any value we please from nothing to infinity. Suppose that the system is referred to rectangular axes $O x, O y$; and that $O P, O M, M P$ make respectively angles $\theta, \theta^{\prime}, \theta^{\prime \prime}$ with $O x$. Hence, by projection on $O y$ and on $O x$, we have

$$
\begin{aligned}
l \sin \theta & =l^{\prime} \sin \theta^{\prime}+l^{\prime \prime} \sin \theta^{\prime \prime}, \\
l \cos \theta & =l^{\prime} \cos \theta^{\prime}+l^{\prime \prime} \cos \theta^{\prime \prime} . \\
\therefore \tan \theta & =\frac{n \sin \theta^{\prime}+\sin \theta^{\prime \prime}}{n \cos \theta^{\prime}+\cos \theta^{\prime \prime}},
\end{aligned}
$$

where $n=l^{\prime} / l^{\prime \prime}$. This result is true whatever be the value of $n$. But $n$ may have any value (ex. gr. $n=\infty$, or $n=0$ ), hence $\tan \theta=\tan \theta^{\prime}=\tan \theta^{\prime \prime}$, which obviously is impossible.

Eighth Fallacy*. Here is a fallacious investigation of the value of $\pi$ : it is founded on well-known quadratures. The area of the semi-ellipse bounded by the minor axis is (in the usual notation) equal to $\frac{1}{2} \pi a b$. If the centre is moved off to an indefinitely great distance along the major axis, the ellipse degenerates into a parabola, and therefore in this particular limiting position the area is equal to two-thirds of the circumscribing rectangle. But the first result is true whatever be the dimensions of the curve.

$$
\begin{aligned}
\therefore \frac{1}{2} \pi a b & =\frac{2}{3} a \times 2 b, \\
\therefore \pi & =8 / 3,
\end{aligned}
$$

a result which obviously is untrue.

Ninth Fallacy. Every ellipse is a circle. The focal distance of a point on an ellipse is given (in the usual notation) in terms

- This was communicated to me by Mr R. Chartres. 
of the abscissa by the formula $r=a+e x$. Hence $d r / d x=e$. From this it follows that $r$ cannot have a maximum or minimum value. But the only closed curve in which the radius vector has not a maximum or minimum value is a circle. Hence, every ellipse is a circle, a result which obviously is untrue.

Geometrical Paradoxes. To the above examples I may add the following questions, which, though not exactly fallacious, lead to results which at a hasty glance appear impossible.

First Paradox. The first is a problem, sent to me by Mr W. Renton, to rotate a plane lamina (say, for instance, a sheet of paper) through four right angles so that the effect is equivalent to turning it through only one right angle.

Second Paradox. As in arithmetic, so in geometry, the theory of probability lends itself to numerous paradoxes. Here is a very simple illustration. A stick is broken at random into three pieces. It is possible to put them together into the shape of a triangle provided the length of the longest piece is less than the sum of the other two pieces ( $c f$. Euc. I. 20), that is, provided the length of the longest piece is less than half the length of the stick. But the probability that a fragment of a stick shall be half the original length of the stick is $1 / 2$. Hence the probability that a triangle can be constructed out of the three pieces into which the stick is broken would appear to be 1/2. This is not true, for actually the probability is $1 / 4$.

Third Paradox. The following example illustrates how easily the eye may be deceived in demonstrations obtained by actually dissecting the figures and re-arranging the parts. In fact proofs by superposition should be regarded with considerable distrust unless they are supplemented by mathematical reasoning. The well-known proofs of the propositions Euclid I. 32 and Euclid I. 47 can be so supplemented and are valid. On the other hand, as an illustration of how deceptive a nonmathematical proof may be, I here mention the familiar paradox that a square of paper, subdivided like a chessboard into 64 small squares, can be cut into four pieces which being put 
together form a figure containing 65 such small squares*. This is effected by cutting the original square into four pieces in the manner indicated by the thick lines in the first figure. If these four pieces are put together in the shape of a rectangle in the way shown in the second figure it will appear as if this rectangle contains 65 of the small squares.

This phenomenon, which in my experience non-mathematicians find perplexing, is due to the fact that the edges of the four pieces of paper, which in the second figure lie along
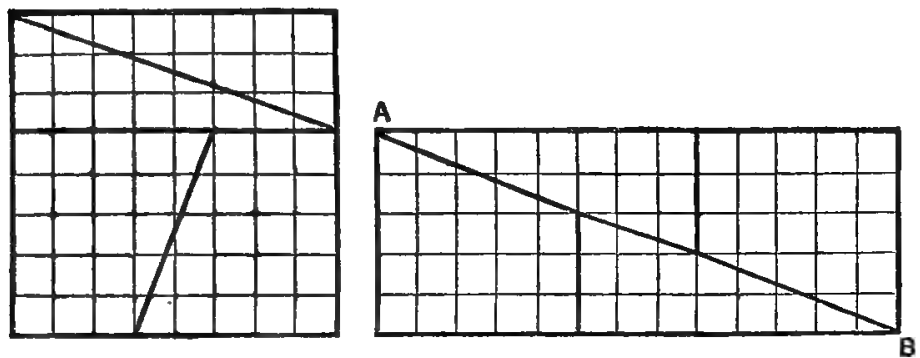

the diagonal $A B$, do not coincide exactly in direction. In reality they include a small lozenge or diamond-shaped figure, whose area is equal to that of one of the 64 small squares in the original square, but whose length $A B$ is much greater than its breadth. The diagrams show that the angle between the two sides of this lozenge which meet at $A$ is $\tan ^{-1} \frac{2}{5}-\tan ^{-1} \frac{8}{8}$, that is, is $\tan ^{-1} \frac{1}{46}$, which is less than $11^{\circ}$. To enable the eye to distinguish so small an angle as this the dividing lines in the first figure would have to be cut with extreme accuracy and the pieces placed together with great care.

This paradox depends upon the relation $5 \times 13-8^{2}=1$. Similar results can be obtained from the formulae

$$
13 \times 34-21^{2}=1,34 \times 89-55^{2}=1, \ldots ;
$$

or from the formulae

$$
5^{2}-3 \times 8=1,13^{2}-8 \times 21=1,34^{2}-21 \times 55=1, \ldots
$$

- I do not know who discovered this paradox. It is given in various modern books, but I cannot find an earlier reference to it than one in the Zeitschrift filr Mathematik und Physik, Leipzig, 1868, vol. xIm, p. 162. Some similar paradozes were given by Ozanam, 1803 edition, rol. I, p. 299. 
These numbers are obtained by finding convergents to the continued fraction

$$
1+\frac{1}{1}+\frac{1}{1}+\frac{1}{1}+\cdots
$$

Dissection Problems. The above paradoxes naturally suggest the consideration of dissection problems. An excellent typical example is to cut a square into 20 equal triangles, and conversely to construct a square of 20 such triangles.

There is an interesting historical example of such a problem. Two late Latin writers, Victorinus and Fortunatianus, describe an Archimedean toy composed of 14 ivory polygons which fitted exactly into a square box, and they suggest that the puzzle was to fit the pieces into the box. A recent discovery* has shown that its association with the name of Archimedes is due to the fact that he gave a construction for dividing a square into 14 such pieces (namely, 11 triangles, 2 scalene quadrilaterals, and one pentagon) so that the area of each piece is a rational fraction of the area of the square. His construction is as follows: let $A B C D$ be the square, and $E, F, G, H$, the midpoints of the sides $A B, B C, C D, D A$. Draw $H B, H F, H C$, and let $J, K, L$ be the mid-points of these lines: draw $A K C$ cutting $H B$ in $M$, and let $N$ be the mid-point of $A M$, and $P$ the midpoint of $B F$. Draw $B N$. Draw $A P$ cutting $H B$ in $Q$. Draw $P J$. Draw $B L$, and produce it to cut $D C$ in $R$. Draw $F L$ cutting $A C$ in $S$. Draw $L G$. Rub out the lines $A Q$ and $B L$. The remaining lines will give a division as required, each figure being an integral multiple of $1 / 48$ th of the square. Why Archimedes propounded so peculiar a division it is impossible to guess, but no doubt the problem has a history of which we are ignorant.

Colouring Maps. I proceed next to mention the geometrical proposition that not more than four colours are necessary in order to colour a map of a country (divided into districts)

* H. Suter, Zeitschrift fur Mathematik und Physik, Abhandlungen zur Gesch. der Math. 1899, vol. xurv, pp. 491-499. 
in such a way that no two contiguous districts shall be of the same colour. By contiguous districts are meant districts having a common line as part of their boundaries : districts which touch only at points are not contiguous in this sense.

The problem was mentioned by A. F. Möbius" in his Lectures in 1840, but it was not until Francis Guthrie† communicated it to De Morgan about 1850 that attention was generally called to it: it is said that the fact had been familiar to practical map-makers for a long time previously. Through De Morgan the proposition then became generally known; and in 1878 Cayley ${ }_{+}^{+}$recalled attention to it by stating that he could not obtain a rigorous proof of it.

Probably the following argument, though not a formal demonstration, will satisfy the reader that the result is true.

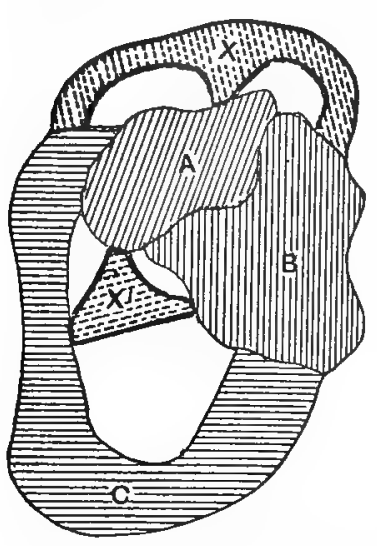

Let $A, B, C$ be three contiguous districts, and let $X$ be any other district contiguous with all of them. Then $X$ must lie either wholly outside the external boundary of the area $A B C$ or wholly inside the internal boundary, that is, it must occupy a position either like $X$ or like $X^{\prime}$. In either case there is no possible way of drawing another area $Y$ which shall be contiguous with $A, B, C$, and $X$. In other words, it is possible to draw on a plane four areas which are contiguous, but it is not possible to draw five such areas. If $A, B, C$ are not contiguous, each with the other, or if $X$ is not contiguous with $A, B$, and $C$, it is not necessary to colour them all differently, and thus the most unfavourable case is that already treated. Moreover any

* Leipzig Transactions (Math.-phys. Classe), 1885, vol. xxxvir, pp. 1-6.

+ See Proceedings of the Royal Society of Edinburgh, July 19, 1880, vol. x, p. 728.

$\ddagger$ Proceedings of the London Mathematical Society, 1878, vol. Ix, p. 148, and Proceedings of the Royal Geographical Society, London, 1879, N.S., vol. I, pp. 259-261, where some of the difficulties are indicated. 
of the above areas may diminish to a point and finally disappear without affecting the argument.

That we may require at least four colours is obvious from the above diagram, since in that case the areas $A, B, C$, and $X$ would have to be coloured differently.

A proof of the proposition involves difficulties of a high order, which as yet have baffled all attempts to surmount them. This is partly due to the fact that if, using only four colours, we build up our map, district by district, and assign definite colours to the districts as we insert them, we can always contrive the addition of two or three fresh districts which cannot be coloured differently from those next to them, and which according]y upset our scheme of colouring. But by starting afresh, it would seem that we can always re-arrange the colours so as to allow of the addition of such extra districts.

The argument by which the truth of the proposition was formerly supposed to be demonstrated was given by A.B. Kempe* in 1879 , but there is a flaw in it.

In 1880, Tait published a solution $f$ depending on the theorem that if a closed network of lines joining an even number of points is such that three and only three lines meet at each point then three colours are sufficient to colour the lines in such a way that no two lines meeting at a point are of the same colour; a closed network being supposed to exclude the case where the lines can be divided into two groups between which there is but one connecting line.

This theorem may be true, if we understand it with the limitation that the network is in one plane and that no line

* He sent his first demonstration across the Atlantic to the American Journal of Mathematics, 1879, vol. II, pp. 193-200; but subsequently he communicated it in simplified forms to the London Mathematical Society, Transactions, 1879, vol. x, pp. 229-231, and to Nature, Feb. 26, 1880, vol. xxI, pp. 399-400. The Hlaw in the argument was indicated in articles by P. J. Heawood in the Quarterly Journal of Mathematics, London, 1890, vol. xxIV, pp. 332-338; and 1897, vol. xxxI, pp. 270-285.

+ Proceedings of the Royal Society of Edinburgh, July 19, 1880, rol, x, p. 729 ; Philosophical Magazine, January, 1884, series 5, vol. xvI, p. 41; and Collected Scientific Papers, Cambridge, vol. II, 1890, p. 93. 
meets any other line except at one of the vertices, which is all that we require for the map theorem; but it has not been proved. Without this limitation it is not correct. For instance the accompanying figure, representing a closed network in three dimensions of 15 lines formed by the sides of two pentagons and the lines joining their corresponding angular points, cannot be coloured as described by Tait. If the figure is in three dimensions, the lines intersect only at the ten vertices of the network. If it is regarded as being in two dimensions, only the ten angular points of the pentagons are treated as vertices of the network, and any other point of intersection of

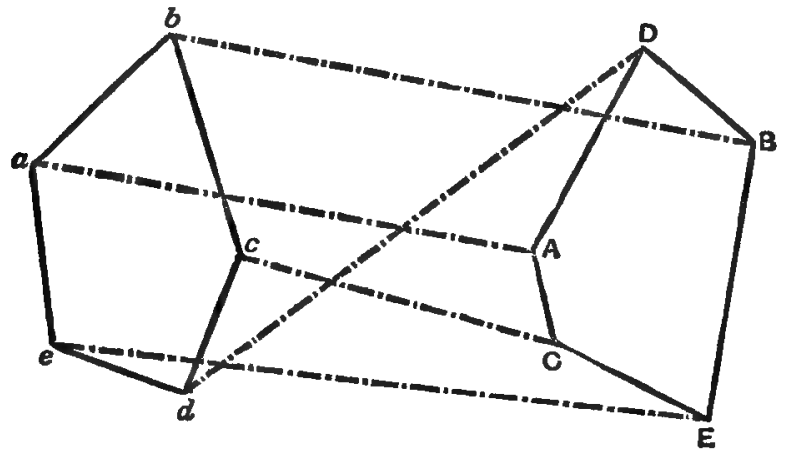

the lines is not regarded as such a vertex. Expressed in technical language the difficulty is this. Petersen* has shown that a graph (or network) of the $2 n$th order and third degree and without offshoots (or feuilles) can be resolved into three graphs of the $2 n$th order and each of the first degree, or into two graphs of the $2 n$th order one being of the first degree and one of the second degree. Tait assumed that the former resolution was the only one possible. The question is whether the limitations mentioned above exclude the second resolution.

Assuming that the theorem as thus limited can be established, Tait's argument that four colours will suffice for a map is divided into two parts and is as follows.

- See J. Petersen of Copenhagen, L'Intermédiaire des Mathematiciens, vol. $\mathbf{v}$, 1898, pp. 225-227; and vol. vI, 1899, pp. 36-38. Also Acta Mathematica, Stookholm, vol. $x \nabla, 1891$, pp. 193-220. 
First, suppose that the boundary lines of contiguous districts form a closed network of lines joining an even number of points such that three and only three lines meet at each point. Then if the number of districts is $n+1$, the number of boundaries will be $3 n$, and there will be $2 n$ points of junction; also by Tait's theorem, the boundaries can be marked with three colours $\beta, \gamma, \delta$ so that no two like colours meet at a point of junction. Suppose this done. Now take four colours, $A, B, C, D$, wherewith to colour the map. Paint one district with the colour $A$; paint the district adjoining $A$ and divided from it by the line $\beta$ with the colour $B$; the district adjoining $A$ and divided from it by the line $\gamma$ with the colour $C$; the district adjoining $A$ and divided from it by the line $\delta$ with the colour $D$. Proceed in this way so that a line $\beta$ always separates the colours $A$ and $B$, or the colours $C$ and $D$; a line $\gamma$ always separates $A$ and $C$, or $D$ and $B$; and a line $\delta$ always separates $A$ and $D$, or $B$ and $C$. It is easy to see that, if we come to a district bounded by districts already coloured, the rule for crossing each of its boundaries will give the same colour: this also follows from the fact that, if we regard $\beta, \gamma, \delta$ as indicating certain operations, then an operation like $\delta$ may be represented as equivalent to the effect of the two other operations $\beta$ and $\gamma$ performed in succession in either order. Thus for such a map the problem is solved.

In the second case, suppose that at any point four or more boundaries meet, then at any such point introduce a small district as indicated below: this will reduce the problem to the first case. The small district thus introduced may be
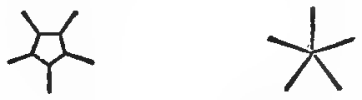

coloured by the previous rule; but after the rest of the map is coloured this district will have served its purpose, it may be then made to contract without limit to a mere point and will disappear leaving the boundaries as they were at first.

Although a proof of the four-colour theorem is still wanting, no one has succeeded in constructing a plane map which requires 
more than four tints to colour it, and there is no reason to doubt the correctness of the statement that it is not necessary to have more than four colours for any plane map. The number of ways in which such a map can be coloured with four tints has been also considered*, but the results are not sufficiently interesting to require mention here.

I believe that in the corresponding question with solids in space of three dimensions not more than six tints are required to colour the exposed surfaces, but I have never seen any attempt to prove this extension of the problem.

Physical Configuration of a Country. As I have been alluding to maps, I may here mention that the theory of the representation of the physical configuration of a country by means of lines drawn on a map was discussed by Cayley and Clerk Maxwell $\uparrow$. They showed that a certain relation exists between the number of hills, dales, passes, \&c. which can co-exist on the earth or on an island. I proceed to give a summary of their nomenclature and conclusions.

All places whose heights above the mean sea level are equal are on the same level. The locus of such points on a map is indicated by a contour-line. Roughly speaking, an island is bounded by a contour-line. It is usual to draw the successive contour-lines on a map so that the difference between the heights of any two successive lines is the same, and thus the closer the contour-lines the steeper is the slope, but the heights are measured dynamically by the amount of work to be done to go from one level to the other and not by linear distances.

A contour-line in general will be a closed curve. This curve may enclose a region of elevation: if two such regions

- See A. C. Dixon, Messenger of Mathematics, Cambridge, 1902-3, vol. zxzIr, pp. 81-83.

† Cayley on 'Contour and Slope Lines,' Philosophical Magazine, London, October, 1859, series 4, vol. xvIr, pp. 264-268; Collected Works, vol. Iv, pp. 108-111. J. Clerk Maxwell on 'Hills and Dales,' Philosophical Magazine, December, 1870, series 4, vol. xL, pp. 421-427; Collected Works, vol. Ir, pp. 233-240. 
meet at a point, that point will be a crunode (i.e. a real double point) on the contour-line through it, and such a point is called a pass. The contour-line may enclose a region of depression: if two such regions meet at a point, that point will be a crunode on the contour-line through it, and such a point is called a fork or bar. As the heights of the corresponding level surfaces become greater, the areas of the regions of elevation become smaller, and at last become reduced to points: these points are the summits of the corresponding mountains. Similarly as the level surface sinks the regions of depression contract, and at last are reduced to points: these points are the bottoms, or immits, of the corresponding valleys.

Lines drawn so as to be everywhere at right angles to the contour-lines are called lines of slope. If we go up a line of slope generally we shall reach a summit, and if we go down such a line generally we shall reach a bottom: we may come however in particular cases either to a pass or to a fork. Districts whose lines of slope run to the same summit are hills. Those whose lines of slope run to the same bottom are dales. A watershed is the line of slope from a summit to a pass or a fork, and it separates two dales. A watercourse is the line of slope from a pass or a fork to a bottom, and it separates two hills.

If $n+1$ regions of elevation or of depression meet at a point, the point is a multiple point on the contour-line drawn through it; such a point is called a pass or a fork of the $n$th order, and must be counted as $n$ separate passes (or forks). If one region of depression meets another in several places at once, one of these must be taken as a fork and the rest as passes.

Having now a definite geographical terminology we can apply geometrical propositions to the subject. Let $h$ be the number of hills on the earth (or an island), then there will be also $h$ summits; let $d$ be the number of dales, then there will be also $d$ bottoms; let $p$ be the whole number of passes, $p_{1}$ that of single passes, $p_{2}$ of double passes, and so on; let $f$ be the whole number of forks, $f_{2}$ that of single forks, $f_{2}$ of double 
forks, and so on; let $w$ be the number of watercourses, then there will be also $w$ watersheds. Hence, by the theorems of Cauchy and Euler,

$$
\text { and } \quad \begin{array}{ll}
h=1+p_{1}+2 p_{2}+\ldots, \\
d=1+f_{1}+2 f_{2}+\ldots, \\
w=2\left(p_{1}+f_{1}\right)+3\left(p_{2}+f_{2}\right)+\ldots
\end{array}
$$

These results can be extended to the case of a multiplyconnected closed surface.

\section{ADDENDUM.}

Note. Page 52. The required rotation of the lamina can be effected thus. Suppose that the result is to be equivalent to turning it through a right angle about a point 0 . Describe on the lamina a square $O A B C$. Rotate the lamina successively through two right angles about the diagonal $O B$ as axis and through two right angles about the side $O A$ as axis, and the required result will be attained. 


\section{CHAPTER IV.}

\section{GEOMETRICAL RECREATIONS CONTINUED.}

Leaving now the question of formal geometrical propositions, I proceed to enumerate a few games or puzzles which depend mainly on the relative position of things, but I postpone to chapter $\mathbf{x}$ the discussion of such amusements of this kind as necessitate any considerable use of arithmetic or algebra. Some writers regard draughts, solitaire, chess, and such like games as subjects for geometrical treatment in the same way as they treat dominoes, backgammon, and games with dice in connection with arithmetic: but these discussions require too many artificial assumptions to correspond with the games as actually played or to be interesting.

The amusements to which I refer are of a more trivial description, and it is possible that a mathematician may like to omit this chapter. In some cases it is difficult to say whether they should be classified as mainly arithmetical or geometrical, but the point is of no importance.

Statical Games of Position. Of the innumerable statical games involving geometry of position I shall mention only three or four.

Three-in-a-row. First, I may mention the game of threein-a-row, of which noughts and crosses, one form of merrilees, and go-bang are well-known examples. These games are played on a board-generally in the form of a square containing $n^{2}$ small squares or cells. The common practice is for one player to place a white counter or piece or to make a cross on each small square or cell which he occupies: his opponent 
similarly uses black counters or pieces or makes a nought on each cell which he occupies. Whoever first gets three (or any other assigned number) of his pieces in three adjacent cells and in a straight line wins. There is no difficulty in giving the complete analysis for boards of 9 cells and of 16 cells : but it is lengthy and not particularly interesting. Most of these games were known to the ancients*, and it is for that reason I mention them here.

Three-in-a-row. Extension. I may, however, add an elegant but difficult extension which has not previously found its way, so far as I am aware, into any book of mathematical recreations. The problem is to place $n$ counters on a plane so as to form as many rows as possible, each of which shall contain three and only three counters $†$.

It is easy to arrange the counters in a number of rows equal to the integral part of $(n-1)^{2} / 8$. This can be effected by the following construction. Let $P$ be any point on a cubic. Let the tangent at $P$ cut the curve again in $Q$. Let the tangent at $Q$ cut the curve in $A$. Let $P A$ cut the curve in $B, Q B$ cut it in $C, P C$ cut it in $D, Q D$ cut it in $E$, and so on. Then the counters must be placed at the points $P, Q, A, B, \ldots$ Thus 9 counters can be placed in 8 such rows; 10 counters in 10 rows; 15 counters in 24 rows; 81 counters in 800 rows; and so on.

If however the point $P$ is a pluperfect point of the $n$th order on the cubic, then Sylvester proved that the above construction gives a number of rows equal to the integral part of $(n-1)(n-2) / 6$. Thus 9 counters can be arranged in 9 rows; 10 counters in 12 rows; 15 counters in 30 rows; and so on.

These, however, are inferior limits and may be exceededfor instance, Sylvester stated that 9 counters can be placed in 10 rows, each containing three counters; I do not know how he placed them, but one way of so arranging them is

* Beok de Fouquieres, Les Jeux des Anciens, second edition, Paris, 1873, chap. IVIII.

† Educational Times Reprints, 1868, vol. vשr, p. 106 ; Ibid. 1886, vol. XL7, pp. 127-128. 
by putting them at points whose coordinates are $(2,0),(2,2)$, $(2,4),(4,0),(4,2),(4,4),(0,0),(3,2),(6,4)$; another way is by putting them at the points $(0,0),(0,2),(0,4),(2,1)$, $(2,2),(2,3),(4,0),(4,2),(4,4)$; more generally, the angular points of a regular hexagon and the three points (at infinity) of intersection of opposite sides form such a group, and therefore any projection of that figure will give a solution. At present it is not possible to say what is the maximum number of rows of three which can be formed from $n$ counters placed on a plane.

Extension to p-in-a-row. The problem mentioned above at once suggests the extension of placing $n$ counters so as to form as many rows as possible, each of which shall contain $p$ and only $p$ counters. Such problems can be often solved immediately by placing at infinity the points of intersection of some of the lines, and (if it is so desired) subsequently projecting the diagram thus formed so as to bring these points to a finite distance. One instance of such a solution is given above.

As examples I may give the arrangement of 10 counters in 5 rows, each containing 4 counters; the arrangement of 16 counters in 15 rows, each containing 4 counters; the arrangement of 18 counters in 9 rows, each containing 5 counters; and the arrangement of 19 counters in 10 rows, each containing 5 counters. These problems I leave to the ingenuity of my readers.

Tesselation. Another of these statical recreations is known as tesselation, and consists in the formation of geometrical designs or mosaics covering a plane area by the use of tiles of given geometrical forms.

If the tiles are regular polygons, the resulting forms can be found by analysis. For instance, if we confine ourselves to the use of like tiles each of which is a regular polygon of $n$ sides, we are restricted to the use of equilateral triangles, squares, or hexagons. For suppose that to fill the space round a point where one of the angles of the polygon is situated we require $m$ polygons. Each interior angle of the polygon is equal to $(n-2) \pi / n$. Hence $m(n-2) \pi / n=2 \pi$. Therefore $(m-2)(n-2)=4$. Now from the nature of the problems $m$ is greater than 2 , and so is $n$. If $m=3, n=6$. If $m>3$, then $n<6$, and since $n>2$, 
we have in this case only to consider the values $n=3, n=4$, and $n=5$. If $n=3$ we have $m=6$. If $n=4$ we have $m=4$. If $n=5, m$ is non-integral, and this is impossible. Thus the only solutions are $m=3$ and $n=6, m=4$ and $n=4, m=6$ and $n=3^{*}$.

If, however, we allow the use of unlike equilateral tiles (triangles, squares, \&cc.), we can construct numerous geometrical designs covering a plane area; though it is impossible to do so by the use of such starred concave polygonst. If at each point the same number and kind of polygons are used, analysis similar to the above shows that we can get six possible superposable arrangements, namely when the polygons are (i) 3-sided, 12-sided, 12-sided; (ii) 4-sided, 6-sided, 12-sided; (iii) 4-sided, 8-sided, 8-sided; (iv) 3-sided, 3-sided, 6-sided, 6-sided; (v) 3-sided, 4-sided, 4-sided, 6-sided; (vi) 3-sided, 3-sided, 3-sided, 4-sided, 4-sided.

The use of colours introduces new considerations. One formation of a pavement by the employment of square tiles of two colours is illustrated by the common chess-board; in this the cells are coloured alternately white and black. Another variety of a pavement made with square tiles of two colours was invented by Sylvester+, who termed it anallagmatic. In the ordinary chess-board, if any two rows or any two columns are placed in juxtaposition, cell to cell, the cells which are side by side are either all of the same colour or all of different colours. In an anallagmatic arrangement, the cells are so coloured (with two colours) that when any two columns or any two rows are placed together side by side, half the cells next to one another are of the same colour and half are of different colours.

Anallagmatic parements composed of $m^{2}$ cells or square tiles can be easily constructed by the repeated use of the four elementary anallagmatic arrangements given in the angular

* Monsieur A. Hermann has proposed an analogous theorem for polygons covering the surface of a sphere.

+ On this, see the second edition of the French translation of this work, Paris, 1908, vol. Ir, pp. 26-37.

‡ See Mathematical Questions from the Educational Times, London, vol. $x$, 1868, pp. 74-76; rol. Iv1, 1892, pp. 97-99. The results are closely connected with theorems in the theory of equations.

B. $\mathbb{R}$. 
spaces of the accompanying diagram. In these fundamental forms $A$ represents one colour and $B$ the other colour. The diamond-shaped figure in the middle of the diagram represents an anallagmatic pavement of 256 tiles which is symmetrical about its diagonals. In half the rows and half the columns each line has 10 white tiles and 6 black tiles, and in the remaining rows and columns each line has 6 white tiles and

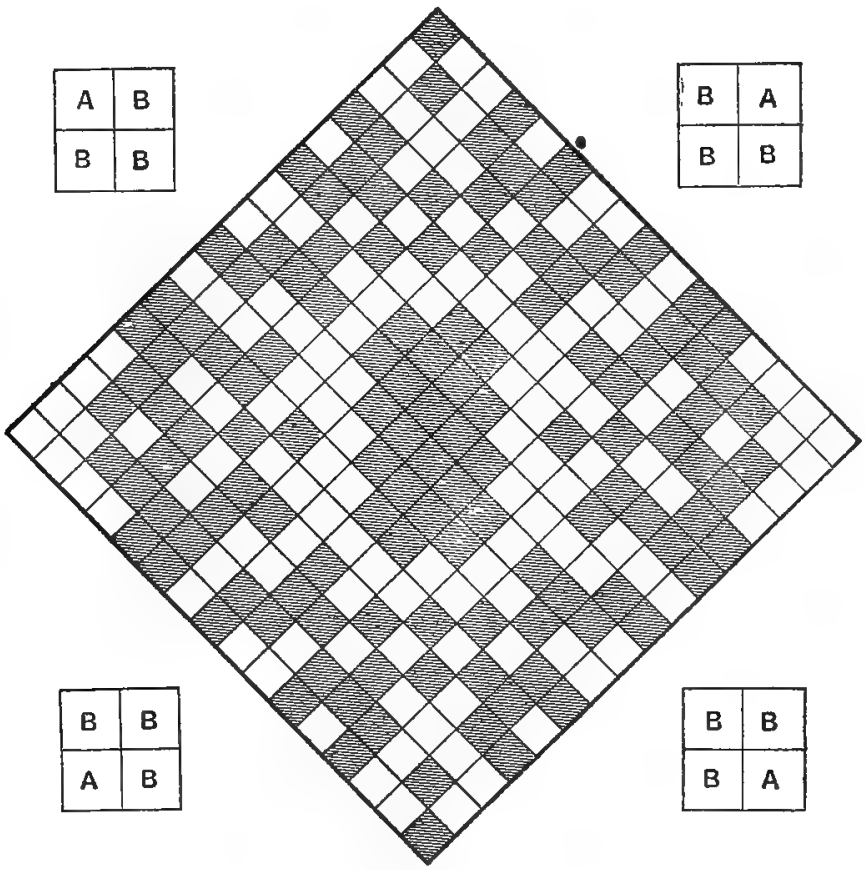

An Anallagmatic Isochromatic Pavement.

10 black tiles. Such an arrangement, where the difference between the number of white and black tiles used in each line is constant, and equal to $\sqrt{m}$, is called isochromatic. If $m$ is odd or oddly even, it is impossible to construct anallagmatic boards which are isochromatic.

Interesting problems can also be proposed when the tiles are triangular, whether equilateral or isosceles right-angled. Two equal isosceles right-angled tiles of different colours can be 
put together so as to make a square tile as shown in the

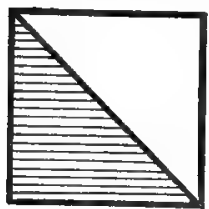
margin We can arrange four such tiles in no less than 256 different ways, making 64 distinct designs. With the use of more tiles the number of possible designs increases with startling rapidity*. I content myself with giving two illustrations of designs of pavements constructed with sixty-four such tiles, all exactly alike.
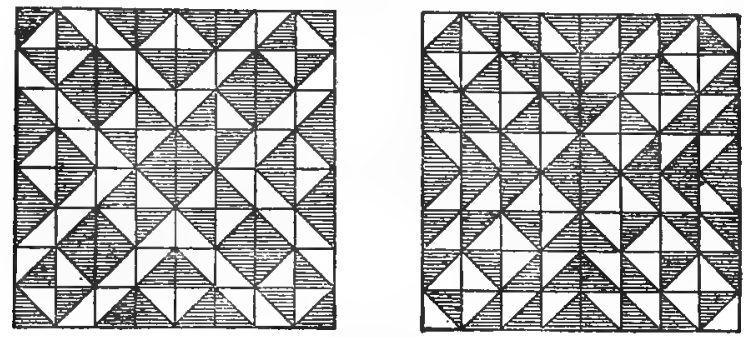

Examples of Tesselated Pavements.

If more than two colours are used, the problems become increasingly difficult. As a simple instance take sixteen square tiles, the upper half of each being yellow, red, pink, or blue, and the lower half being gold, green, black, or white, no two tiles being coloured alike. Such tiles can be arranged in the form of a square so that in each vertical, horizontal, and diagonal line there shall be 8 colours and no more; or so that there shall be 6 colours and no more; or 5 colours and no more; or 4 colours and no more.

Colour-Cube Problem. As an example of a recreation analogous to tesselation I will mention the colour-cube problem $\dagger$. Stripped of mathematical technicalities the problem may be enunciated as follows. A cube has six faces, and if six colours are chosen we can paint each face with a different colour. By permuting the order of the colours we can obtain

* On this, see Lucss, Récréations Mathématiques, Paris, 1882-3, vol. п, part 4; heresfter I shall refer to this work by the name of the author.

† P. A. MacMahon, London Mathematical Society Proceedings, vol. xxIv, 1893, pp. 145-155; and New Mathematical Pastimes, Cambridge, 1921, pp. $42-46$. 
thirty such cubes, no two of which are coloured alike. Take any one of these cubes, $K$, then it is desired to select eight out of the remaining twenty-nine cubes, such that they can be arranged in the form of a cube (whose linear dimensions are double those of any of the separate cubes) coloured like the cube $K$, and placed so that where any two cubes touch each other the faces in contact are coloured alike.

Only one collection of eight cubes can be found to satisfy these conditions. These eight cubes can be determined by the following rule. Take any face of the cube $K$ : it has four angles, and at each angle three colours meet: By permuting the colours cyclically we can obtain from each angle two other cubes, and the eight cubes so obtained are those required. A little consideration will show that these are the required cubes, and that the solution is unique.

For instance suppose that the six colours are indicated by the letters $a, b, c, d, e, f$. Let the cube $K$ be put on a table, and to fix our ideas suppose that the face coloured $f$ is at the bottom, the face coloured $a$ is at the top, and the faces coloured $b, c, d$, and $e$ front respectively the east, north, west, and south points of the compass. I may denote such an arrangement by $(f ; a ; b, c, d, e)$. One cyclical permutation of the colours which meet at the north-east corner of the top face gives the cube $(f ; c ; a, b, d, e)$, and a second cyclical permutation gives the cube $(f ; b ; c, a, d, e)$. Similarly cyclical permutations of the colours which meet at the northwest corner of the top face of $K$ give the cubes $(f ; d ; b, a, c, e)$ and $(f ; c ; b, d, a, e)$. Similarly from the top south-west corner of $K$ we get the cubes $(f ; e ; b, c, a, d)$ and $(f ; d ; b, c, e, a)$ : and from the top south-east corner we get the cubes $(f ; e ; a, c, d, b)$ and $(f ; b ; e, c, d, a)$.

The eight cubes being thus determined it is not difficult to arrange them in the form of a cube coloured similarly to $K$, and subject to the condition that faces in contact are coloured alike; in fact they can be arranged in two ways to satisfy these conditions. One such way, taking the cubes in the numerical order given above, is to put the cubes $3,6,8$, and 2 
at the SE, NE, NW, and SW corners of the bottom face; of course each placed with the colour $f$ at the bottom, while 3 and 6 have the colour $b$ to the east, and 2 and 8 have the colour $d$ to the west: the cubes $7,1,4$, and 5 will then form the SE, NE, NW, and SW corners of the top face; of course each placed with the colour $a$ at the top, while 7 and 1 have the colour $b$ to the east, and 5 and 4 have the colour $d$ to the west. If $K$ is not given, the difficulty of the problem is increased. Similar puzzles in two dimensions can be made.

TANGRams. The formation of designs by means of seven pieces of wood, namely, a square, a rhombus, and five triangles, known as tans, of fixed traditional shapes, is one of the oldest amusements in the East. Many hundreds of figures representing men, women, birds, beasts, fish, houses, boats, domestic objects, designs, \&c. can be made, but the recreation is not mathematical, and I reluctantly content myself with a bare mention of it.

Drnamical Games of Positron. Games which are played by moving pieces on boards of various shapes—such as merrilees, fox and geese, solitaire, backgammon, draughts, and chesspresent more interest. In general, possible movements of the pieces are so numerous that mathematical analysis is not practicable, but in a few games the possible movements are sufficiently limited as to permit of mathematical treatment; one or two of these are given later: here I shall confine myself mainly to puzzles and simple amusements.

Shunting Problems. The first I will mention is a little puzzle which I bought some years ago and which was described as the "Great Northern Puzzle." It is typical of a good many problems connected with the shunting of trains, and though it rests on a most improbable hypothesis, I give it as a specimen of its kind.

The puzzle shows a railway, $D E F$, with two sidings, $D B A$ and $F C A$, connected at $A$. The portion of the rails at $A$ which is common to the two sidings is long enough to permit of a single wagon, like $P$ or $Q$, running in or out of it; but is too short to contain the whole of an engine, like $R$. Hence, if 
an engine runs up one siding, such as $D B A$, it must come back the same way.

Initially a small block of wood, $P$, coloured to represent a wagon, is placed at $B$; a similar block, $Q$, is placed at $C$; and a longer block of wood, $R$, representing an engine, is placed at $E$. The problem is to use the engine $R$ to interchange the wagons $P$ and $Q$, without allowing any flying shunts.

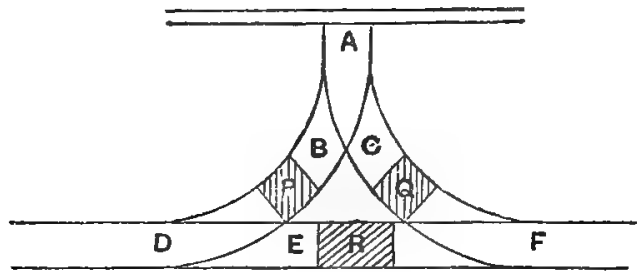

Another shunting puzzle, on sale in the streets in 1905, under the name of the "Chifu-Chemulpo Puzzle," is made as follows. A loop-line $B G E$ connects two points $B$ and $E$ on a railway track $A F$, which is supposed blocked at both ends, as shown in the diagram. In the model, the track $A F^{\prime}$ is 9 inches long, $A B=E F=1 \frac{5}{6}$ inches, and $A H=F K=B C=D E=\frac{1}{4}$ inch.

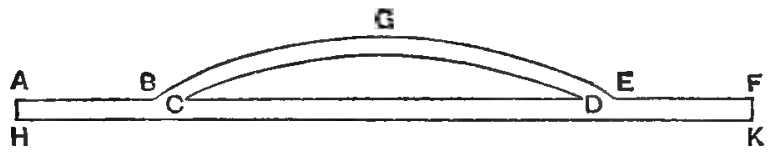

On the track and loop are eight wagons, numbered successively 1 to 8 , each one inch long and one-quarter of an inch broad, and an engine, $\boldsymbol{e}$, of the same dimensions. Originally the wagons are on the track from $A$ to $F$ and in the order $1,2,3,4,5,6,7,8$, and the engine is on the loop. The construction and the initial arrangement ensure that at any one time there cannot be more than eight vehicles on the track. Also if eight vehicles are on it only the penultimate vehicle at either end can be moved on to the loop, but if less than eight are on the track then the last two vehicles at either end can be moved on to the loop. If the points at each end of the loopline are clear, it will hold four, but not more than four, vehicles. The object is to reverse the order of the wagons on the track, 
so that from $A$ to $F$ they will be numbered successively 8 to 1 ; and to do this by means which will involve as few transferences of the engine or a wagon to or from the loop as is possible. Twenty-six moves are required, and there is more than one solution in 26 moves.

Other shunting problems are not uncommon, but these two examples will suffice.

Ferry-Boat Problems. Everybody is familiar with the story of the showman who was travelling with a wolf, a goat, and a basket of cabbages; and for obvious reasons was unable to leave the wolf alone with the goat, or the goat alone with the cabbages. The only means of transporting them across a river was a boat so small that he could take in it only one of them at a time. The problem is to show how the passage could be effected*.

A somewhat similar problem is to arrange for the passage of a river by three men and three boys who have the use of a boat which will not carry at one time more than one man or two boys. Fifteen passages are required $\uparrow$.

Problems like these were proposed by Alcuin, Tartaglia, and other medieval writers. The following is a common type of such questions. Three $\ddagger$ beautiful ladies have for husbands three men, who are young, gallant, and jealous. The party are travelling, and find on the bank of a river, over which they have to pass, a small boat which can hold no more than two persons. How can they cross the river, it being agreed that, in order to avoid scandal, no woman shall be left in the society of a man unless her husband is present? Eleven passages are required. With two married couples five passages are required. The similar problem with four married couples is insoluble.

Another similar problem is the case of $n$ married couples who have to cross a river by means of a boat which can be rowed by one person and will carry $n-1$ people, but not more, with the condition that no woman is to be in the society of a man unless her husband is present. Alcuin's problem given

* Ozanam, 1803 edition, vol. I, p. 171; 1840 edition, p. 77.

† H. E. Dudeney, The Tribune, October 4, 1906.

‡ Bachet, Appendix, problem rv, p. 212. 
above is the case of $n=3$. Let $y$ denote the number of passages from one bank to the other which will be necessary. Then it has been shown that if $n=3, y=11$; if $n=4, y=9$; and if $n>4, y=7$.

The following analogous problem is due to the late $\mathrm{E}$. Lucas*. To find the smallest number $x$ of persons that a boat must be able to carry in order that $n$ married couples may by its aid cross a river in such a manner that no woman shall remain in the company of any man unless her husband is present; it being assumed that the boat can be rowed by one person only. Also to find the least number of passages, say $y$, from one bank to the other which will be required. M. Delannoy has shown that if $n=2$, then $x=2$, and $y=5$. If $n=3$, then $x=2$, and $y=11$. If $n=4$, then $x=3$, and $y=9$. If $n=5$, then $x=3$, and $y=11$. And finally if $n>5$, then $x=4$, and $y=2 n-1$.

M. De Fonteney has remarked that, if there was an island in the middle of the river, the passage might be always effected by the aid of a boat which could carry only two persons. If there are only two or only three couples the island is unnecessary, and the case is covered by the preceding method. His solution, involving $8 n-8$ passages, is as follows. The first nine passages will be the same, no matter how many couples there may be: the result is to transfer one couple to the island and one couple to the second bank. The result of the next eight passages is to transfer one couple from the first bank to the second bank, this series of eight operations must be repeated as often as necessary until there is left only one couple on the first bank, only one couple on the island, and all the rest on the second bank. The result of the last seven passages is to transfer all the couples to the second bank. It would however seem that if $n$ is greater than 3 , we need not require more than $6 n-7$ passages from land to land + .

M. G. Tarry has suggested an extension of the problem, which still further complicates its solution. He supposes that

* Lucas, vol. i, pp. 15-18, 237-238.

† See H. E. Dudeney, Amusenents in Mathematics, London, 1917, p. 237. 
each husband travels with a harem of $m$ wives or concubines; moreover, as Mohammedan women are brought up in seclusion, it is reasonable to suppose that they would be unable to row a boat by themselves without the aid of a man. But perhaps the difficulties attendant on the travels of one wife may be deemed sufficient for Christians, and I content myself with merely mentioning the increased anxieties experienced by Mohammedans in similar circumstances.

Geodesics. Geometrical problems connected with finding the shortest routes from one point to another on a curved surface are often difficult, but geodesics on a flat surface or flat surfaces are in general readily determinable.

I append one instance*, though $I$ should have hesitated to do so, had not experience shown that some readers do not readily see the solution. It is as follows. A room is 30 feet long, 12 feet wide, and 12 feet high. On the middle line of one of the smaller side walls and one foot from the ceiling is a wasp. On the middle line of the opposite wall and 11 feet from the ceiling is a fly. The wasp catches the fly by crawling all the way to it: the fly, paralysed by fear, remaining still. The problem is to find the shortest route that the wasp can follow.

To obtain a solution we observe that we can cut a sheet of paper so that, when folded properly, it will make a model to scale of the room. This can be done in several ways. If, when the paper is again spread out flat, we can join the points representing the wasp and the fly by a straight line lying wholly on the paper we shall obtain a geodesic route between them. Thus the problem is reduced to finding the way of cutting out the paper which gives the shortest route of the lind.

Here is the diagram corresponding to a solution of the above question, where $A$ represents the floor, $B$ and $D$ the longer side-walls, $C$ the ceiling, and $W$ and $F$ the positions on the two smaller side-walls occupied initially by the wasp and fly.

- This is due to Mr H. E. Dudeney. I heard a similar question propounded at Cambridge in 1903, but I first sav it in print in the Daily Mlail, London, February 1, 1905. 
In the diagram the square of the distance between $W$ and $F$ is $(32)^{2}+(24)^{2}$; hence the distance is 40 feet.

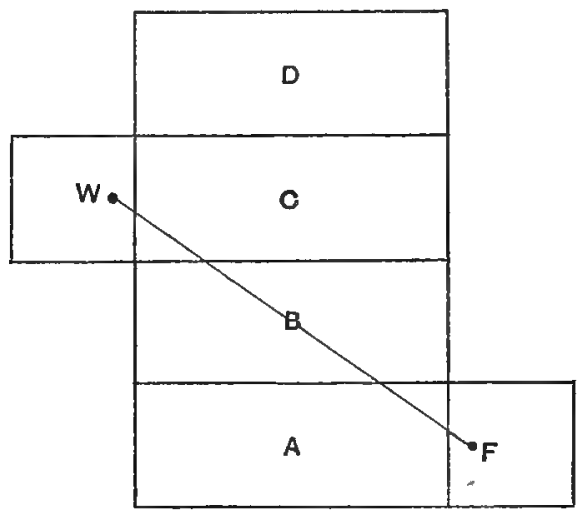

Problems with Counters placed in a row. Numerous dynamical problems and puzzles may be illustrated with a box of counters, especially if there are counters of two colours. Of course coins or pawns or cards will serve equally well. I proceed to enumerate a few of these played with counters placed in a row.

First Problem with Counters. The following problem must be familiar to many of my readers. Ten counters (or coins) are placed in a row. Any counter may be moved over two of those adjacent to it on the counter next beyond them. It is required to move the counters according to the above rule so that they shall be arranged in five equidistant couples.

If we denote the counters in their initial positions by the numbers $1,2,3,4,5,6,7,8,9,10$, we proceed as follows. Put 7 on 10 , then 5 on 2 , then 3 on 8 , then 1 on 4, and lastly 9 on 6. Thus they are arranged in pairs on the places originally occupied by the counters $2,4,6,8,10$.

Similarly by putting 4 on 1 , then 6 on 9 , then 8 on 3 , then 10 on 7 , and lastly 2 on 5 , they are arranged in pairs on the places originally occupied by the counters $1,3,5,7,9$.

If two superposed counters are reckoned as only one, solutions analogous to those given above will be obtained by 
putting 7 on 10 , then 5 on 2 , then 3 on 8 , then 1 on 6 , and lastly 9 on 4 ; or by putting 4 on 1 , then 6 on 9 , then 8 on 3 , then 10 on 5 , and lastly 2 on $7^{*}$.

There is a somewbat similar game played with eight counters, but in this case the four couples finally formed are not equidistant. Here the transformation will be effected if we move 5 on 2 , then 3 on 7 , then 4 on 1 , and lastly 6 on 8 . This form of the game is applicable equally to $(8+2 n)$ counters, for if we move 4 on 1 we have left on one side of this couple a row of $(8+2 n-2)$ counters. This again can be reduced to one of $(8+2 n-4)$ counters, and in this way finally we have left eight counters which can be moved in the way explained above.

A more complete generalization would be the case of $n$ counters, where each counter might be moved over the $m$ counters adjacent to it on to the one beyond them. For instance we may place twelve counters in a row and allow the moving a counter over three adjacent counters. By such movements we can obtain four piles, each pile containing three counters. Thus, if the counters be numbered consecutively, one solution can be obtained by moving 7 on 3 , then 5 on 10, then 9 on 7 , then 12 on 8 , then 4 on 5 , then 11 on 12 , then 2 on 6 , and then 1 on 2 . Or again we may place sixteen counters in a row and allow the moving a counter over four adjacent counters on to the next counter available. By such movements we can get four piles, each pile containing four counters. Thus, if the counters be numbered consecutively, one solution can be obtained by moving 8 on 3 , then 9 on 14, then 1 on 5 , then 16 on 12 , then 7 on 8 , then 10 on 7 , then 6 on 9 , then 15 on 16 , then 13 on 1 , then 4 on 15, then 2 on 13, and then 11 on 6 .

Second Problem with Counters. Another problemt, of a somewhat similar kind, is of Japanese origin. Place four florins (or white counters) and four halfpence (or black counters) alternately in a line in contact with one another. It is required

- Note by J. Fitzpatrick to a French translation of the third edition of this work, Paris, 1898.

† Bibliotheca Mathematica, 1896, series 3, vol. vI, p. 323 ; P. G. Tait, Philo. sophical Magazine, London, January, 1884, series 5, vol. 8VIr, p. 39; or Collected Scientific Papers, Cambridge, vol. II, 1890, p. 93. 
in four moves, each of a pair of two contiguous pieces, without altering the relative position of the pair, to form a continuous line of four halfpence followed by four florins.

This can be solved as follows. Let a florin be denoted by $a$ and a halfpenny by $b$, and let $\times \times$ denote two contiguous blank spaces. Then the successive positions of the pieces may be represented thus:

Initially + . $\quad \times \times a b a b a b a b$.
After the first move - $\quad b a a b a b a \times \times b$.
After the second move $\quad b a a b \times \times a a b b$.
After the third move. $\quad b \times \times b a a a a b b$.
After the fourth move $\quad b b b b a a a a \times \times$.

The operation is conducted according to the following rule. Suppose the pieces to be arranged originally in circular order, with two contiguous blank spaces, then we always move to the blank space for the time being that pair of coins which occupies the places next but one and next but two to the blank space on one assigned side of it.

A similar problem with $2 n$ counters - $n$ of them being white and $n$ black-will at once suggest itself, and, if $n$ is greater than 4 , it can be solved in $n$ moves. I have however failed to find a simple rule which covers all cases alike, but solutions, due to M. Delannoy, have been given* for the four cases where $n$ is of the form $4 m, 4 m+2,4 m+1$, or $4 m+3$; in the first two cases the first $\frac{1}{2} n$ moves are of pairs of dissimilar counters and the last $\frac{1}{2} n$ moves are of pairs of similar counters; in the last two cases, the first move is similar to that given above, namely, of the penultimate and antepenultimate counters to the beginning of the row, the next $\frac{1}{2}(n-1)$ moves are of pairs of dissimilar counters, and the final $\frac{1}{2}(n-1)$ moves are of similar counters.

The problem is also capable of solution if we substitute the restriction that at each move the pair of counters taken up must be moved to one of the two ends of the row instead of the condition that the final arrangement is to be continuous. 
Tait suggested a variation of the problem by making it a condition that the two coins to be moved shall also be made to interchange places; in this form it would seem that five moves are required; or, in the general case, $n+1$ moves are required.

Problems on a Chess-board with Counters or Pawns. The following three problems require the use of a chess-board as well as of counters or pieces of two colours. It is more convenient to move a pawn than a counter, and if therefore $I$ describe them as played with pawns it is only as a matter of convenience and not that they have any connection with chess. The first is characterized by the fact that in every position not more than two moves are possible; in the second and third problems not more than four moves are possible in any position. With these limitations, analysis is possible. I shall not discuss the similar problems in which more moves are possible.

First Problem with Pawns*. On a row of seven squares on a chess-board 3 white pawns (or counters), denoted in the diagram by " $a$ "s, are placed on the 3 squares at one end, and 3 black pawns (or counters), denoted by " $b$ "s, are placed on the 3 squares at the other end-the middle square being left vacant. Each piece can move only in one direction; the " $a$ " pieces can move from left to right, and the " $b$ " pieces from right to left. If the square next to a piece is unoccupied, it can move on

\begin{tabular}{|l|l|l|l|l|l|l|}
\hline$a$ & $a$ & $a$ & & $b$ & $b$ & $b$ \\
\hline
\end{tabular}

to that; or if the square next to it is occupied by a piece of the opposite colour and the square beyond that is unoccupied, then it can, like a queen in draughts, leap over that piece on to the unoccupied square beyond it. The object is to get all the white pawns in the places occupied initially by the black pawns and vice versa.

The solution requires 15 moves. It may be effected by moving first a white pawn, then successively two black pawns, then three white pawns, then three black pawns, then three white pawns, then two black pawns, and then one white pawn. We can express this solution by saying that if we number the

* Lucas, vol. n, part 5, pp. 141-143. 
cells (a term used to describe each of the small squares on a chess-board) consecutively, then initially the vacant space occupies the cell 4 and in the successive moves it will occupy the cells $3,5,6,4,2,1,3,5,7,6,4,2,3,5,4$. Of these moves, six are simple and nine are leaps.

More generally, if we have $m$ white pawns at one end of a row of $m+n+1$ cells, and $n$ black pawns at the other end, the arrangement can be reversed in $m n+m+n$ moves, of which $m+n$ are simple and $m n$ are leaps.

Second Problem with Pawns*. A similar game may be played on a rectangular or square board. The case of a square board containing 49 cells, or small squares, will illustrate this sufficiently: in this case the initial position is shown in the annexed diagram where the " $a$ "s denote the pawns or pieces

\begin{tabular}{|c|c|c|c|c|c|c|}
\hline$a$ & $a$ & $a$ & $a$ & $b$ & $b$ & $b$ \\
\hline$a$ & $a$ & $a$ & $\frac{a}{a}$ & $\frac{b}{b}$ & $\frac{b}{b}$ \\
\hline$a$ & $\frac{a}{a}$ & $\frac{a}{a}$ & $\frac{b}{b}$ & $\frac{b}{b}$ \\
\hline$a$ & $a$ & $\frac{a}{a}$ & & $\frac{b}{b}$ & $\frac{b}{}$ \\
\hline$a$ & $\frac{a}{a}$ & $\frac{a}{b}$ & $\frac{b}{b}$ & $\frac{b}{b}$ \\
\hline$a$ & $a$ & $a$ & $b$ & $b$ & $b$ & $b$ \\
\hline$a$ & $a$ & $\frac{b}{b}$ & $b$ & $b$ & $b$ & $b$ \\
\hline
\end{tabular}

of one colour, and the " $b$ "s those of the other colour. The " $a$ " pieces can move horizontally from left to right or vertically down, and the " $b$ pieces can move horizontally from right to left or vertically up, according to the same rules as before.

The solution reduces to the preceding case. The pieces in the middle column can be interchanged in 15 moves. In the course of these moves every one of the seven cells in that column is at some time or other vacant, and whenever that 
is the case the pieces in the row containing the vacant cell can be interchanged. To interchange the pieces in each of the seven rows will require 15 moves. Hence to interchange all the pieces will require $15+(7 \times 15)$ moves, that is, 120 moves.

If we place $2 n(n+1)$ white pawns and $2 n(n+1)$ black pawns in a similar way on a square board of $(2 n+1)^{2}$ cells, we can transpose them in $2 n(n+1)(n+2)$ moves: of these $4 n(n+1)$ are simple and $2 n^{2}(n+1)$ are leaps.

Third Problem with Pawns. The following analogous problem is somewhat more complicated. On a square board of 25 cells, place eight white pawns or counters on the cells

\begin{tabular}{|l|l|l|l|l|}
\hline$a$ & $b$ & $c$ & & \\
\hline$d$ & $e$ & $f$ & & \\
\hline$g$ & $\frac{}{h}$ & $*$ & $H$ & $G$ \\
\hline & & $F$ & $\frac{G}{E}$ & $\frac{D}{A}$ \\
\hline & & $C$ & $B$ & \\
\hline
\end{tabular}

denoted by small letters in the annexed diagram, and eight black pawns or counters on the cells denoted by capital letters: the cell marked with an asterisk (*) being left blank. Each pawn can move according to the laws already explained-the white pawns being able to move only horizontally from left to right or vertically downwards, and the black pawns being able to move only horizontally from right to left or vertically upwards. The object is to get all the white pawns in the places initially occupied by the black pawns and vice versa. No moves outside the dark line are permitted.

Since there is only one cell on the board which is unoccupied, and since no diagonal moves and no backward moves are permitted, it follows that at each move not more than two pieces of either colour are capable of moving. There are however a very large number of empirical solutions. In previous editions I have given a symmetrical solution in 48 moves, but the following, due to $\mathrm{Mr}$ H. E. Dudeney, is effected in 46 moves : 
the letters indicating the cells from which the pieces are successively moved. It will be noticed that the first twenty-three moves lead to a symmetrical position, and that the next twentytwo moves can be at once obtained by writing the first twentytwo moves in reverse order and interchanging small and capital letters. Similar problems with boards of various shapes can be easily constructed.

Probably, were it worth the trouble, the mathematical theory of games such as that just described might be worked out by the use of Vandermonde's notation, described later in chapter VI, or by the analogous method employed in the theory of the game of solitaire*.

Problems on a Chess-board with Chess-pieces. There are several mathematical recreations with chess-pieces, other than pawns. Some of these are given later in chapter VI.

Geometrical Puzzles with Rods, etc. Another species of geometrical puzzles, to which here I will do no more than allude, are made of steel rods, or of wire, or of wire and string. Numbers of these are often sold in the streets of London for a penny each, and some of them afford ingenious problems in the geometry of position. Most of them could hardly be discussed without the aid of diagrams, but they are inexpensive to construct, and in fact innumerable puzzles on geometry of position can be made with a couple of stout sticks and a ball of string, or with only a box of matches: several examples are given in various recent English works. Most of them exemplify the difficulty of mentally realizing the effect of geometrical alterations in a figure unless they are of the simplest character.

Paradromic Rings. The fact just stated is illustrated by the familiar experiment of making paradromic rings by cutting a paper ring prepared in the following manner.

* On the theory of the solitaire, see Reiss, 'Beiträge zur Theorie des SolitärSpiels,' Crelle's Journal, Berlin, 1858, vol. LIv, pp. 344-379; and Lucas, vol. I, part $\nabla$, pp. 89-1+1. 
Take a strip of paper or piece of tape, say, for convenience, an inch or two wide and at least nine or ten inches long, rule a line in the middle down the length $A B$ of the strip, gum one end over the other end $B$, and we get a ring like a section of a cylinder. If this ring is cut by a pair of scissors along the ruled line we obtain two rings exactly like the first, except that they are only half the width. Next suppose that the end $A$ is twisted through two right angles before it is gummed to $B$ (the result of which is that the back of the strip at $A$ is gummed over the front of the strip at $B$ ), then a cut along the line will produce only one ring. Next suppose that the end $A$ is twisted once completely round (i.e. through four right angles) before it is gummed to $B$, then a similar cut produces two interlaced rings. If any of my readers think that these results could be predicted off-hand, it may be interesting to them to see if they can predict correctly the effect of again cutting the rings formed in the second and third experiments down their middle lines in a manner similar to that above described.

The theory is due to J. B. Listing* who discussed the case when the end $A$ receives $m$ half-twists, that is, is twisted through $m \pi$, before it is gummed to $B$.

If $m$ is even we obtain a surface which has two sides and two edges, which are termed paradromic. If the ring is cut along a line midway between the edges, we obtain two rings, each of which has $m$ half-twists, and which are linked together $\frac{1}{2} m$ times.

If $m$ is odd we obtain a surface having only one side and one edge. If this ring is cut along its mid-line, we obtain only one ring, but it has $2 m$ half-twists, and if $m$ is greater than unity it is knotted.

\section{ADDENDUM.}

Note. Page 64. One method of arranging 16 counters in 15 lines, as stated in the text, is as follows. Draw a regular re-entrant pentagon vertices $A_{1}, A_{2}, A_{3}, A_{4}, A_{6}$, and centre $O$. The sides intersect in five

*Vorstudien zur Topologie, Die Studien, Göttingen, 1847, part 又. 
points $B_{1}, \ldots B_{6}$. These latter points may be joined so as to form a smaller regular re-entrant pentagon whose sides intersect in five points $C_{1}, \ldots C_{6}$. The 16 points indicated are arranged as desired (The Canterbury Puzzles, 1907, p. 140).

An arrangement of 18 counters in 9 rows, each containing 5 counters, can be obtained thus. From one angle, $A$ of an equilateral triangle $A A^{\prime} A^{\prime \prime}$, draw lines $A D, A E$ inside the triangle making any angles with $A A^{\prime}$. Draw from $A^{\prime}$ and $A^{\prime \prime}$ lines similarly placed in regard to $A^{\prime} A^{\prime \prime}$ and $A^{\prime \prime} A$. Let $A^{\prime} D^{\prime}$ cut $A^{\prime \prime} E^{\prime \prime}$ in $F$, and $A^{\prime} E^{\prime}$ cut $A^{\prime \prime} D^{\prime \prime}$ in $G$. Then $A F G$ is a straight line. The 3 vertices of the triangle and the 15 points of intersection of $A D, A E, A F$, with the similar pencils of lines drawn from $A^{\prime}, A^{\prime \prime}$, will give an arrangement as required.

An arrangement of 19 counters in 10 rows, each containing 5 counters, can be obtained by placing counters at the 19 points of intersection of the 10 lines $x= \pm a, x= \pm b, y= \pm a, y= \pm b, y= \pm x$ : of these points two are at infinity.

Note. Page 69. The Great Northern Shunting Problem is effected thus. (i) $R$ pushes $P$ into $A$. (ii) $R$ returns, pushes $Q$ up to $P$ in $A$, couples $Q$ to $P$, draws them both out to $F$, and then pushes them to $E$. (iii) $A$ is now uncoupled, $R$ takes $Q$ back to $A$, and loaves it there. (iv) $R$ returns to $P$, takes $P$ back to $C$, and leaves it there. ( $v) R$ running successively through $F, D, B$ comes to $A$, draws $Q$ out, and leaves it at $B$.

Note. Page 70. One solution of the Chifu-Chemulpo Puzzle is as follows. Move successively wagons 2, 3, 4 up, i.e. on to the loop line. [Then push 1 along the straight track close to 5 ; this is not a "move."] Next, move 4 down, i.e. on to the straight track and push it along to 1 . Next, move 8 up, 3 down to the end of the track and keep it there temporarily, 6 up, 2 down, $e$ down, 3 up, 7 up. [Then push 5 to the end of the track and keep it there temporarily.] Next, move 7 down, 6 down, 2 up, 4 up. [Then push $e$ along to 1.] Next, move 4 down to the end of the track and keep it there temporarily, 2 down, 5 up, 3 down, 6 up, 7 up, 8 down to the end of the track, $e$ up, 5 down, 6 down, 7 down. In this solution we moved $e$ down to the track at one end, then shifted it along the track, and finally moved it up to the loop from the other end of the track. We might equally well move $e$ down to the track at one end, and finally move it back to the loop from the same end. In this solution the pieces successively moved are $2,3,4,4, e, 8,7,3,2,6,5,5,6,3,2,7,2,5,6,3$, $7, e, 8,5,6,7$. 


\section{CHAPTER V.}

\section{MECHANICAL RECREATIONS.}

I proceed now to enumerate a few questions connected with mechanics which lead to results that seem to me interesting from a historical point of view or paradoxical. Problems in mechanics generally involve more difficulties than problems in arithmetic, algebra, or geometry, and the explanations of some phenomena-such as those connected with the flight of birdsare still incomplete, while the explanations of many others of an interesting character are too difficult to find a place in a nontechnical work. Here I exclude all transcendental mechanics, and confine myself to questions which, like those treated in the preceding chapters, are of an elementary character. The results are well-known to mathematicians.

I assume that the reader is acquainted with the fundamental ideas of kinematics and dynamics, and is familiar with the three Newtonian laws; namely, first that a body will continue in its state of rest or of uniform motion in a straight line unless compelled to change that state by some external force : second, that the change of momentum per unit of time is proportional to the external force and takes place in the direction of it: and third, that the action of one body on another is equal in magnitude but opposite in direction to the reaction of the second body on the first. The first and second laws state the principles required for solving any question on the motion of a particle under the action of given forces. The third law supplies the additional principle required for the solution of problems in which two or more particles influence one another. 
Motron. The difficulties connected with the idea of motion have been for a long time a favourite subject for paradoxes, some of which bring us into the realm of the philosophy of mathematics.

Zeno's Paradoxes on Motion. One of the earliest of these is the remark of Zeno to the effect that since an arrow cannot move where it is not, and since also it cannot move where it is (that is, in the space it exactly fills), it follows that it cannot move at all. This is sometimes presented in the form that at each instant a flying arrow occupies a fixed position; but occupying a fixed position at a given instant means that it is then at rest. Hence the arrow is at rest at every instant of its flight, and therefore is not in motion. The usual answer is that the very idea of the motion of the arrow implies the passage from where it is to where it is not.

Zeno also asserted that the idea of motion was itself inconceivable, for what moves must reach the middle of its course before it reaches the end. Hence the assumption of motion presupposes another motion, and that in turn another, and so ad infinitum. His objection was in fact analogous to the biological difficulty expressed by Swift:-

"So naturalists observe, a flea hath smaller fleas that on him prey.

And these have smaller fleas to bite 'em. And so proceed ad infinitum."

Or as De Morgan preferred to put it

"Great fleas have little fleas upon their backs to bite 'em,

And little fleas have lesser fleas, and so ad infinitum.

And the great fleas themselves, in turn, have greater fleas to go on;

While these have greater still, and greater still, and so on."

Achilles and the Tortoise. Zeno's paradox about Achilles and the tortoise is known even more widely. The assertion was that if Achilles ran ten times as fast as a tortoise, yet if the tortoise had (say) 1000 yards start it could never be overtaken. To establish this, Zeno argued that when Achilles had gone the 1000 yards, the tortoise would still be 100 yards in front of him; by the time he had covered these 100 yards, it would still be 10 yards in front of him; and so on for ever. Thus Achilles would get nearer and nearer to the tortoise but would never overtake it. Zeno regarded this as confirming his view that the popular idea of motion is self-contradictory. 
The fallacy lies in the use of the word "never." The argument shows that during the time occupied by the motion described Achilles will not reach the tortoise. It does not deal with what happens after that time, and in fact Achilles would then overtake and pass the tortoise. Probably Zeno would have stated that the argument and explanation alike rest on the assumption, which he would not have admitted, that space and time are infinitely divisible.

Zeno's Paradox on Time. Zeno seems further to have contended that while, to an accurate thinker, the notion of the infinite divisibility of time was impossible, it was equally impossible to think of a minimum measure of time. For suppose, he argued, that $\tau$ is the smallest conceivable interval, and suppose that three horizontal lines composed of three consecutive spans $a b c, a^{\prime} b^{\prime} c^{\prime}, a^{\prime \prime} b^{\prime \prime} c^{\prime \prime}$ are placed so that $a, a^{\prime}, a^{\prime \prime}$ are vertically over one another, as also $b, b^{\prime}, b^{\prime \prime}$ and $c, c^{\prime}, c^{\prime \prime}$. Imagine the second line moved as a whole one span to the right in the time $\tau$, and simultaneously the third line moved as a whole one span to the left. Then $b, a^{\prime}, c^{\prime \prime}$ will be vertically over one another. And in this duration $\tau$ (which by hypothesis is indivisible) $a^{\prime}$ must have passed vertically over the space $a^{\prime \prime} b^{\prime \prime}$ and the space $b^{\prime \prime} c^{\prime \prime}$. Hence the duration is divisible, contrary to the hypothesis.

The Paradox of Tristram Shandy. Mr Russell has enunciated* a paradox somewhat similar to that of Achilles and the Tortoise, save that the intervals of time considered get longer and longer during the course of events. Tristram Shandy, as we know, took two years writing the history of the first two days of his life, and lamented that, at this rate, material would accumulate faster than he could deal with it, so that he could never finish the work, however long he lived. But had he lived long enough, and not wearied of his task, then, even if his life had continued as eventfully as it began, no part of his biography would have remained unwritten. For if he wrote the events of the first day in the first year, he would write the - B. A. W. Russell, Principles of Mathematics, Cambridge, 1903, vol. 1, p. 358. 
events of the $n$th day in the $n$th year, hence in time the events of any assigned day would be written, and therefore no part of his biography would remain unwritten. This argument might be put in the form of a demonstration that the part of a magnitude may be equal to the whole of it.

Questions, such as those given above, which are concerned with the continuity of space and time involve difficulties of a high order. Many of the resulting perplexities are due to the assumption that the number of things in a collection of them is greater than the number in a part of that collection. This is axiomatic for a finite number of things, but must not be assumed as being necessarily true of infinite collections.

Angular Motion. A non-mathematician finds additional difficulties in the idea of angular motion. For instance, there is a well-known proposition on motion in an equiangular spiral which shows that a body, moving with uniform velocity and as slowly as we please, may in a finite time whirl round a fixed point an infinite number of times. To a non-mathematician the result seems paradoxical if not impossible.

The demonstration is as follows. The equiangular spiral is the trace of a point $P$, which moves along a line $O P$, the line $O P$ turning round a fixed point $O$ with uniform angular velocity while the distance of $P$ from $O$ decreases with the time in geometrical progression. If the radius vector rotates through four right angles we have one convolution of the curve. All convolutions are similar, and the length of each convolution is a constant fraction, say $1 / n$ th, that of the convolution immediately outside ib. Inside any given convolution there are an infinite number of convolutions which get smaller and smaller as we get nearer the pole. Now suppose a point $Q$ to move uniformly along the spiral from any point towards the pole. If it covers the first convolution in $a$ seconds, it will cover the next in $a / n$ seconds, the next in $a / n^{2}$ seconds, and so on, and will finally reach the pole in

$$
\left(a+a / n+a / n^{2}+a / n^{3}+\ldots \ldots \ldots\right)
$$

seconds, that is, in $a n /(n-1)$ seconds. The velocity is uniform, 
and yet in a finite time, $Q$ will have traversed an infinite number of convolutions and therefore have circled round the pole an infinite number of times*.

Simple Relative Motion. Even if the philosophical difficulties suggested by Zeno are settled or evaded, the mere idea of relative motion has been often found to present difficulties, and Zeno himself failed to explain a simple phenomenon involving the principle. As one of the easiest examples of this kind, I may quote the common question of how many trains going from $B$ to $A$ a passenger from $A$ to $B$ would meet and pass on his way, assuming that the journey either way takes $4 \frac{1}{2}$ hours and that the trains start from each end every hour. The answer is 9. Or again, take two pennies, face upwards on a table and edges in contact. Suppose that one is fixed and that the other rolls on it without slipping, making one complete revolution round it and returning to its initial position. How many revolutions round its own centre has the rolling coin made? The answer is 2.

Laws of Motion. I proceed next to make a few remarks on points connected with the laws of motion.

The first law of motion is often said to define force, but it is in only a qualified sense that this is true. Probably the meaning of the law is best expressed in Clifford's phrase, that force is "the description of a certain kind of motion"in other words it is not an entity but merely a convenient way of stating, without circumlocution, that a certain kind of motion is observed.

It is not difficult to show that any other interpretation lands us in difficulties. Thus some authors use the law to justify a definition that force is that which moves a body or changes its motion; yet the same writers speak of a steamengine moving a train. It would seem then that, according to them, a steam-engine is a force. That such statements are current may be fairly reckoned among mechanical paradoxes.

* The proposition is put in this form in J. Richord's Philosophie des Mathematiques, Paris, 1903, pp. 119-120. 
The idea of force is difficult to grasp. How many people, for instance, could predict correctly what would happen in a question as simple as the following? A rope (whose weight may be neglected) hangs over a smooth pulley; it has one end fastened to a weight of 10 stone, and the other end to a sailor of weight 10 stone, the sailor and the weight hanging in the air. The sailor begins steadily to climb up the rope; will the weight move at all; and, if so, will it rise or fall ? In fact, it will rise.

It will be noted that in the first law of motion it is asserted that, unless acted on by an external force, a body in motion continues to move (i) with uniform velocity, and (ii) in a straight line.

The tendency of a body to continue in its state of rest or of uniform motion is called its inertia. This tendency may be used to explain various common phenomena and experiments. Thus, if a number of dominoes or draughts are arranged in a vertical pile, a sharp horizontal blow on one of those near the bottom will send it out of the pile, and those above will merely drop down to take its place-in fact they have not time to change their relative positions before there is sufficient space for them to drop vertically as if they were a solid body. On this principle depends the successful performance of numerous mechanical tricks and puzzles.

The statement about inertia in the first law may be taken to imply that a body set in rotation about a principal axis passing through its centre of mass will continue to move with a uniform angular velocity and to keep its axis of rotation fixed in direction. The former of these statements is the assumption on which our measurement of time is based as mentioned below in chapter Xx. The latter assists us to explain the motion of a projectile in a resisting fluid. It affords the explanation of why the barrel of a rifle is grooved; and why, similarly, anyone who has to throw a flat body of irregular shape (such as a card) in a given direction usually gives it a rapid rotatory motion about a principal axis. Elegant illustrations of the fact just mentioned are afforded by a good many of the tricks of acrobats, 
though the full explanation of most of them also introduces other considerations. Thus it is a common feat to toss on to the top surface of an umbrella a penny so that it alights on its edge, and then, by turning round the stick of the umbrella rapidly, to cause the coin to rotate. By twisting the umbrella at the proper rate, the coin can be made to appear stationary and standing upright, though the umbrella is moving away underneath it, while by diminishing or increasing the angular velocity of the umbrella the penny can be made to run forwards or backwards. This is not a difficult trick to execute: it was introduced by Japanese conjurers.

The tendency of a body in motion to continue to move in a straight line is sometimes called its centrifugal force. Thus, if a train is running round a curve, it tends to move in a straight line, and is constrained only by the pressure of the rails to move in the required direction. Hence it presses on the outer rail of the curve. This pressure can be diminished to some extent both by raising the outer rail, and by putting a guard rail, parallel and close to the inner rail, against which the wheels on that side also will press.

An illustration of this fact occurred in a little known incident of the American civil war*. In the spring of 1862 a party of volunteers from the North made their way to the rear of the Southern armies and seized a train, intending to destroy, as they passed along it, the railway which was the main line of communication between various confederate corps and their base of operations. They were however detected and pursued. To save themselves, they stopped on a sharp curve and tore up some rails so as to throw the engine which was following them off the line. Unluckily for themselves they were ignorant of dynamics and tore up the inner rails of the curve, an operation which did not incommode their pursuers, who were travelling at a high speed.

The second law gives us the means of measuring mass, force, and therefore work. A given agent in a given time can do only a definite amount of work. This is illustrated by the

* Capturing a Locomotive by W. Pittenger, London, 1882, p. 104. 
fact that although, by means of a rigid lever and a fixed fulcrum, any force however small may be caused to move any mass however large, yet what is gained in power is lost in speed-as the popular phrase runs.

Montucla* inserted a striking illustration of this principle founded on the well-known story of Archimedes who is said to have declared to Hiero that, were he but given a fixed fulcrum, he could move the world. Montucla assumed that a man could work incessantly at the rate of 116 foot-lbs. per second, which is a very high estimate. On this assumption it would take over three billion centuries, i.e. $3 \times 10^{14}$ years, before a particle whose mass was equal to that of the earth was moved as much as one inch against gravity at the surface of the earth: to move it one inch along a horizontal plane on the earth's surface would take about 6,000 centuries.

Stability of Equilibrium. It is known to all those who have read the elements of mechanics that the centre of gravity of a body, which is resting in equilibrium under its own weight, must be vertically above its base: also, speaking generally, we may say that, if every small displacement has the effect of raising the centre of gravity, then the equilibrium is stable, that is, the body when left to itself will return to its original position; but, if a displacement has the effect of lowering the centre of gravity, then for that displacement the equilibrium is unstable; while, if every displacement does not alter the height above some fixed plane of the centre of gravity, then the equilibrium is neutral. In other words, if in order to cause a displacement work has to be done against the forces acting on the body, then for that displacement the equilibrium is stable, while if the forces do work the equilibrium is unstable.

A good many of the simpler mechanical toys and tricks afford illustrations of this principle.

Magic Bottlest. Among the most common of such toys are the small bottles-trays of which may be seen any day in the streets of London-which keep always upright, and cannot

* Ozanam, 1803 edition, vol. Ir, p. 18 ; 1840 edition, p. 202.

+ Ozanam, 1803 edition, vol. II, p. 15; 1840 edition, p. 201. 
be upset until their owner orders them to lie down. Such a bottle is made of thin glass or varnished paper fixed to the plane surface of a solid hemisphere or smaller segment of a sphere. Now the distance of the centre of gravity of a homogeneous hemisphere from the centre of the sphere is three-eighths of the radius, and the mass of the glass or varnished paper is so small compared with the mass of the lead base that the centre of gravity of the whole bottle is still within the hemisphere. Let us denote the centre of the hemisphere by $C$, and the centre of gravity of the bottle by $G$.

If such a bottle is placed with the hemisphere resting on a horizontal plane and $G C$ vertical, any small displacement on the plane will tend to raise $G$, and thus the equilibrium is stable. This may be seen also from the fact that when slightly displaced there is brought into play a couple, of which one force is the reaction of the table passing through $C$ and acting vertically upward, and the other the weight of the bottle acting vertically downward at $G$. If $G$ is below $C$, this couple tends to restore the bottle to its original position.

If there is dropped into the bottle a shot or nail so heavy as to raise the centre of gravity of the whole above $C$, then the equilibrium is unstable, and, if any small displacement is given, the bottle falls over on to its side.

Montucla says that in his time it was not uncommon to see boxes of tin soldiers mounted on lead hemispheres, and when the lid of the box was taken off the whole regiment sprang to attention.

In a similar way we may explain how to balance a pencil in a vertical position, with its point resting on the top of one's finger, an experiment which is described in nearly every book of puzzles*. This is effected by taking a penknife, of which one blade is opened through an angle of (say) $120^{\circ}$, and sticking the blade in the pencil so that the handle of the penknife is below the finger. The centre of gravity is thus brought below the point of support, and a small displacement given to the

* Ex. gr. Oughtred, Mathematicall Recreations, p. 24; Ozanam, 1803 edition, vol. Ir, p. 14 ; 1840 edition, p. 200. 
pencil will raise the centre of gravity of the whole: thus the equilibrium is stable.

Other similar tricks are the suspension of a bucket over the edge of a table by a couple of sticks, and the balancing of a coin on the edge of a wine-glass by the aid of a couple of forks*-the sticks or forks being so placed that the centre of gravity of the whole is vertically below the point of support and its depth below it a maximum.

The toy representing a horseman, whose motion continually brings him over the edge of a table into a position which seems to ensure inmediate destruction, is constructed in somewhat the same way. A wire has one end fixed to the feet of the rider; the wire is curved downwards and backwards, and at the other end is fixed a weight. When the horse is placed so that his hind legs are near the edge of the table and his forefeet over the edge, the weight is under his hind feet. Thus the whole toy forms a pendulum with a curved instead of a straight rod. Hence the farther it swings over the table, the higher is the centre of gravity raised, and thus the toy tends to return to its original position of equilibrium.

An elegant modification of the prancing horse was brought out at Paris in 1890 in the shape of a toy made of tin and in the figure of a mant. The legs are pivoted so as to be movable about the thighs, but with a wire check to prevent too long a step, and the hands are fastened to the top of a $n$-shaped wire weighted at its ends. If the figure is placed on a narrow sloping plank or strip of wood passing between the legs of the $\mathrm{n}$, then owing to the $\mathrm{n}$-shaped wire any lateral displacement of the figure will raise its centre of gravity, and thus for any such displacement the equilibrium is stable. Hence, if a slight lateral disturbance is given, the figure will oscillate and will rest alternately on each foot: when it is supported by one foot the other foot under its own weight moves forwards, and thus the figure will walk down the plank though with a slight reeling motion.

* Oughtred, p. 30; Ozanam, 1803 edition, vol. II, p. 12; 1840 edition, p. 199.

+ La Nature, Paris, March, 1891. 
Columbus's Egg. The toy known as Columbus's egg depends on the same principle as the magic bottle, though it leads to the converse result. The shell of the egg is made of tin and cannot be opened. Inside it and fastened to its base is a hollow truncated tin cone, and there is also a loose marble inside the shell. If the egg is held properly, the marble runs inside the cone and the egg will stand on its base, but so long as the marble is outside the cone, the egg cannot be made to stand on its base.

Cones running up hill*. Another common experiment, which produces the optical effect of a body moving by itself up an inclined plane, also depends on the tendency of a body to take a position so that its centre of gravity is as low as possible. Usually the experiment is performed as follows. Arrange two sticks in the shape of a $V$, with the apex on a table and the two upper ends resting on the top edge of a book placed on the table. Take two equal cones fixed base to base, and place them with the curved surfaces resting on the sticks near the apex of the $\mathbf{V}$, the common axis of the cones being horizontal and parallel to the edge of the book. Then, if properly arranged, the cones will run up the plane formed by the sticks.

The explanation is obvious. The centre of gravity of the cones moves in the vertical plane midway between the two sticks and it occupies a lower position as the points of contact on the sticks get farther apart. Hence as the cone rolls up the sticks its centre of gravity descends.

Test of Internal Structure. Here is another simple experiment of a somewhat different character. Suppose two balls constructed of equal size and weight, one of lead and hollow in the middle, the other of copper and solid; and suppose that both spheres are gilt so that in weight, appearance, and elasticity they are indistinguishable. How can we tell which of them is solid? The answer is by allowing them to roll down a rough inclined plane, side by side.

Perpetual Motion. The idea of making a machine which once set going would continue to go for ever by itself has been

- Ozanam, 1803 edition, vol. Ir, p. 49; 1840 edition, p. 216. 
the ignis fatuus of self-taught mechanicians in much the same way as the quadrature of the circle has been that of self-taught geometricians.

Now the obvious meaning of the third law of motion is that a force is only one aspect of a stress, and that whenever a force is caused another equal and opposite one is brought also into existence-though it may act upon a different body, and thus be immaterial for the particular problem considered. The law however is capable of another interpretation*, namely, that the rate at which an agent does work (that is, its action) is equal to the rate at which work is done against it (that is, its reaction). If it is allowable to include in the reaction the rate at which kinetic energy is being produced, and if work is taken to include that done against molecular forces, then it follows from this interpretation that the work done by an agent on a system is equivalent to the total increase of energy, that is, the power of doing work. Hence in an isolated system the total amount of energy is constant. If this is granted, then since friction and some molecular dissipation of energy cannot be wholly prevented, it must be impossible to construct in an isolated system a machine capable of perpetual motion.

I do not propose to describe in detail the various machines for producing perpetual motion which have been suggested $\dagger$, but the machine described below will serve to illustrate one of the assumptions commonly made by these inventors.

The machine to which I refer consists of two concentric vertical wheels in the same plane, and mounted on a horizontal axle through their centre, $C$. The space between the wheels is divided into compartments by spokes inclined at a constant angle to the radii to the points whence they are drawn, and each compartment contains a heavy bullet. This will be clear from the diagram. Apart from these bullets, the wheels would be in equilibrium. Each bullet tends to turn the wheels round their axle, and the moment which measures this tendency is

- Newton's Principles, last paragraph of the Scholium to the Laws of Motion.

† Several of them have been described in H. Dirck's Perpetuum Mobile, Londen, 1861, 2nd edition, 1870. 
the product of the weight of the bullet and its distance from the vertical through $C$.

The idea of the constructors of such machines was that, as the bullet in any compartment would roll under gravity to the lowest point of the compartment, the bullets on the right-hand side of the wheel in the diagram would be farther from the vertical through $C$ than those on the left. Hence the sum of

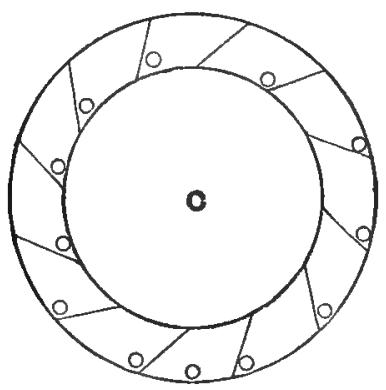

the moments of the weights of the bullets on the right would be greater than the sum of the moments of those on the left. Thus the wheels would turn continually in the same direction as the hands of a watch. The fallacy in the argument is obvious.

Another large group of machines for producing perpetual motion depended on the use of a magnet to raise a mass which was then allowed to fall under gravity. Thus, if the bob of a simple pendulum was made of iron, it was thought that magnets fixed near the highest points which were reached by the bob in the swing of the pendulum would draw the bob up to the same height in each swing and thus give perpetual motion, but the inventors omitted to notice that the bob of the pendulum would gradually get magnetised.

Of course it is only in isolated systems that the total amount of energy is constant, and, if a source of external energy can be obtained from which energy is continually introduced into the system, perpetual motion is, in a sense, possible; though even here materials would ultimately wear out. Streams, wind, the 
solar heat, and the tides are among the more obvious of such sources.

There was at Paris in the latter half of the eighteenth century a clock which was an ingenious illustration of such perpetual motion*. The energy which was stored up in it to maintain the motion of the pendulum was provided by the expansion of a silver rod. This expansion was caused by the daily rise of temperature, and by means of a train of levers it wound up the clock. There was a disconnecting apparatus, so that the contraction due to a fall of temperature produced no effect, and there was a similar arrangement to prevent overwinding. I believe that a rise of eight or nine degrees Fahrenheit was sufficient to wind up the clock for twenty-four hours.

By utilizing the rise and fall of the barometer, James Cox, a London jeweller of the eighteenth century, produced, in an analogous way, a clock $\uparrow$ which ran continuously without winding up.

I have in my possession a watch which produces the same effect by somewhat different means. Inside the case is a steel weight, and if the watch is carried in a pocket this weight rises and falls at every step one takes, somewhat after the manner of a pedometer. The weight is raised by the action of the person who has it in his pocket in taking a step, and in falling it winds up the spring of the watch. On the face is a small dial showing the number of hours for which the watch is wound up. As soon as the hand of this dial points to fifty-six hours, the train of levers which winds up the watch disconnects automatically, so as to prevent overwinding the spring, and it reconnects again as soon as the watch has run down eight hours. The watch is an excellent time-keeper, and a walk of about a couple of miles is sufficient to wind it up for twenty-four hours.

- Ozanam, 1803 edition, vol. II, p. 105 ; 1840 edition, p. 238.

+ A full description of the mechanism will be found in the English Mechanic, April 30, 1909, pp. 288-289. 
Models. I may add here the observation, which is well known to mathematicians, but is a perpetual source of disappointment to ignorant inventors, that it frequently happens that an accurate model of a machine will work satisfactorily while the machine itself will not do so.

One reason for this is as follows. If all the parts of a model are magnified in the same proportion, say $m$, and if thereby a line in it is increased in the ratio $m: 1$, then the areas and volumes in it will be increased respectively in the ratios $m^{2}: 1$ and $m^{8}: 1$. For example, if the side of a cube is doubled then a face of it will be increased in the ratio $4: 1$ and its volume will be increased in the ratio $8: 1$.

Now if all the linear dimensions are increased $m$ times. then some of the forces that act on a machine (such, for example, as the weight of part of it) will be increased $m^{3}$ times, while others which depend on area (such as the sustaining power of a beam) will be increased only $m^{2}$ times. Hence the forces that act on the machine and are brought into play by the various parts may be altered in different proportions, and thus the machine may be incapable of producing results similar to those which can be produced by the model.

The same argument has been adduced in the case of animal life to explain why very large specimens of any particular breed or species are usually weak. For example, if the linear dimensions of a bird were increased $n$ times, the work necessary to give the power of flight would have to be increased no less than $n^{7}$ times* Again, if the linear dimensions of a man of height $5 \mathrm{ft} .10 \mathrm{in}$. were increased by one-seyenth his height would become $6 \mathrm{ft}$. 8 in., but his weight would be increased in the ratio $512: 343$ (i.e. about half as much again), while the cross sections of his legs, which would have to bear this weight, would be increased only in the ratio $64: 49$; thus in some respects he would be less efficient than before. Of course the increased dimensions, length of limb, or size of muscle might be of greater advantage than the relative loss of strength; hence the problem of what are the most efficient

- Helmholtz, Gesammelte Abhandlungen, Leipzig, 1881, vol. I, p. 165.

B. $\mathbf{R}$. 
proportions is not simple, but the above argument will serve to illustrate the fact that the working of a machine may not be similar to that of a model of it.

Leaving now these elementary considerations I pass on to some other mechanical questions.

SAILING QUICKER THAN THE WIND. As a kinematical paradox I may allude to the possibility of sailing quicker than the wind blows, a fact which strikes many people as curious.

The explanation* depends on the consideration of the velocity of the wind relative to the boat. Perhaps, however, a non-mathematician will find the solution simplified if I consider first the effect of the wind-pressure on the back of the sail which drives the boat forward, and second the resistance to motion caused by the sail being forced through the air.

When the wind is blowing against a plane sail the resultant pressure of the wind on the sail may be resolved into two components, one perpendicular to the sail (but which in general is not a function only of the component velocity in that direction, though it vanishes when that component vanishes) and the other parallel to its plane. The latter of these has no effect on the motion of the ship. The component perpendicular to the sail tends to move the ship in that direction. This pressure, normal to the sail, may be resolved again into two components, one in the direction of the keel of the boat, the other in the direction of the beam of the boat. The former component drives the boat forward, the latter to leeward. It is the object of a boat-builder to construct the boat on lines so that the resistance of the water to motion forward shall be as small as possible, and the resistance to motion in a perpendicular direction (i.e. to leeward) shall be as large as possible; and I will assume for the moment that the former of these resistances may be neglected, and that the latter is so large as to render motion in that direction impossible.

Now, as the boat moves forward, the pressure of the air on the front of the sail will tend to stop the motion. As

* Ozanam, 1803 edition, vol. III, pp. 359, 367; 1840 edition, pp. 540, 543. 
long as its component normal to the sail is less than the pressure of the wind behind the sail and normal to it, the resultant of the two will be a force behind the sail and normal to it which tends to drive the boat forwards. But as the velocity of the boat increases, a time will arrive when the pressure of the wind is only just able to balance the resisting force which is caused by the sail moving throngh the air. The velocity of the boat will not increase beyond this, and the motion will be then what mathematicians describe as "steady."

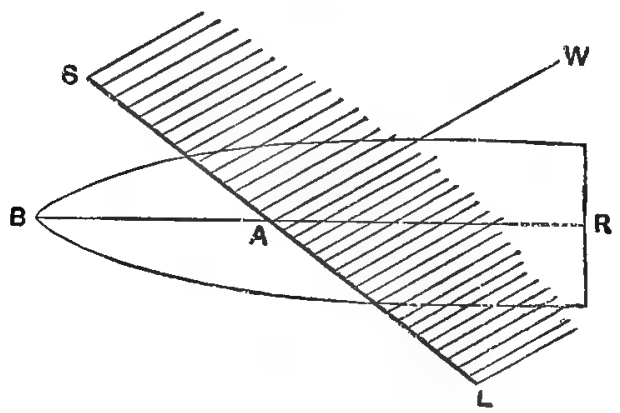

In the accompanying figure, let $B A R$ represent the keel of a boat, $B$ being the bow, and let $S A L$ represent the sail. Suppose that the wind is blowing in the direstion WA with a velocity $u$; and that this direction makes an angle $\theta$ with the keel, i.e. angle $W A R=\theta$. Suppose that the sail is set su as to make an angle $\alpha$ with the keel, i.e. angle $B A S=\alpha$, and therefore angle $W A L=\theta+\alpha$. Suppose finally that $v$ is the velocity of the boat in the direction $A B$.

I have already shown that the solution of the problem depends on the relative directions and velocities of the wind and the boat; hence to find the result reduce the boat to rest by impressing on it a velocity $v$ in the direction $B A$. The resultant velocity of $v$ parallel to $B A$ and of $u$ parallel to $W A$ will be parallel to $S L$, if $v \sin \alpha=u \sin (\theta+\alpha)$; and in this cass the resultant pressure perpendicular to the sail vacishes.

Thus, for steady motion we have $v \sin \alpha=u \sin (\theta+\alpha)$. Hence, whenever $\sin (\theta+\alpha)>\sin \alpha$, we have $v>u$. Suppose, 
to take one instance, the sail to be fixed, that is, suppose $a$ to be a constant. Then $v$ is a maximum if $\theta+\alpha=\frac{1}{2} \pi$, that is, if $\theta$ is equal to the complement of $\alpha$. In this case we have $v=u \operatorname{cosec} \alpha$, and therefore $v$ is greater than $u$. Hence, if the wind makes the same angle $\alpha$ abaft the beam that the sail makes with the keel, the velocity of the boat will be greater than the velocity of the wind.

Next, suppose that the boat is running close to the wind, so that the wind is before the beam (see figure below), then in the same way as before we have $v \sin \alpha=u \sin (\theta+\alpha)$, or $v \sin \alpha=u \sin \phi$, where $\phi=$ angle $W A S=\pi-\theta-\alpha$. Hence $v=u \sin \phi \operatorname{cosec} \alpha$.

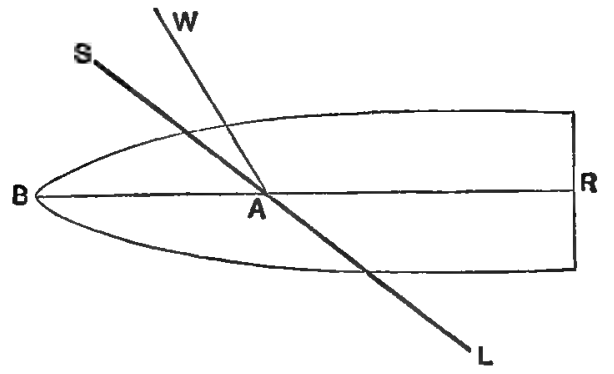

Let $w$ be the component velocity of the boat in the teeth of the wind, that is, in the direction $A W$. Then we have $w=v \cos B A W=v \cos (\alpha+\phi)=u \sin \phi \operatorname{cosec} x \cos (\alpha+\phi)$. If $\alpha$ is constant, this is a maximum when $\phi=\frac{1}{4} \pi-\frac{1}{2} \alpha$; and, if $\phi$ has this value, then $w=\frac{1}{2} u(\operatorname{cosec} a-1)$. This formula shows that $w$ is greater than $u$, if $\sin a<\frac{1}{3}$. Thus, if the sails can be set so that $\alpha$ is less than $\sin ^{-1} \frac{1}{3}$, that is, rather less than $19^{\circ} 29^{\prime}$, and if the wind has the direction above assigned, then the component velocity of the boat in the face of the wind is greater than the velocity of the wind.

The above theory is curious, but it must be remembered that in practice considerable allowance has to be made for the fact that no boat for use on water can be constructed in which the resistance to motion in the direction of the keel can be wholly neglected, or which would not drift slightly to leeward if the wind was not dead astern Still this makes less 
difference than might be thought by a landsman. In the case of boats sailing on smooth ice the assumptions made are substantially correct, and the practical results are said to agree closely with the theory.

BoAt MOVED BY a Rope. There is a form of boat-racing, occasionally used at regattas, which affords a somewhat curious illustration of certain mechanical principles. The only thing supplied to the crew is a coil of rope, and they have, without leaving the boat, to propel it from one point to another as rapidly as possible. The motion is given by tying one end of the rope to the after thwart, and giving the other end a series of violent jerks in a direction parallel to the keel. I am told that in still water a pace of two or three miles an hour can be thus attained.

The chief cause for this result seems to be that the friction between the boat and the water retards all relative motion, but it is not great enough to affect materially motion caused by a sufficiently big impulse. Hence the usual movements of the crew in the boat do not sensibly move the centre of gravity of themselves and the boat, but this does not apply to an impulsive movement, and if the crew in making a jerk move their centre of gravity towards the bow $n$ times more rapidly than it returus after the jerk, then the boat is impelled forwards at least $n$ times more than backwards: hence on the whole the motion is forwards.

Motion of Fluids and Motion in Fluids. The theories of motion of fluids and motion in fluids involve considerable difficulties. 'Here I will mention only one or two instaucesmainly illustrations of Hauksbee's Law.

Hauksbee's Law. When a fluid is in motion the pressure is less than when it is at rest*. Thus, if a current of air is

* See Besant, Hydromechanics, Cambridge, 1867, art. 149, where however it is assumed that the pressure is proportional to the density. Hauksbee was the earliest writer whe called attention to the problem, but I do not know who first explained the phenomenon; some references to it are given by Willis, Cambridge Philosophical 'Transactions, 1830, vol. III, pp. 129-140. 
moving in a tube, the pressure on the sides of the tube is less than when the air is at rest-and the quicker the air moves the smaller is the pressure. This fact was noticed by Hauksbee nearly two centuries ago. In an elastic perfect fluid in which the pressure is proportional to the density, the law connecting the pressure, $p$, and the steady velocity, $v$, is $p=\Pi \alpha^{-v^{2}}$, where $\Pi$ and $\alpha$ are constants : the establishment of the corresponding formula for gases where the pressure is proportional to a power of the density presents no difficulty.

'The principle is illustrated by a twopenny toy, on sale in most toy-shops, called the pneumatic mystery. This consists of a tube, with a cup-shaped end in which rests a wooden ball. If the tube is held in a vertical position, with the mouthpiece at the upper end and the cup at the lower end, then, if anyone blows hard through the tube and places the ball against the cup, the ball will remain suspended there. The explanation is that the pressure of the air below the ball is so much greater than the pressure of the air in the cup that the ball is held up.

The same effect may be produced by fastening to one end of a tube a piece of cardboard having a small hole in it. If a piece of paper is placed over the hole and the experimenter blows through the tube, the paper will not be detached from the card but will bend so as to allow the egress of the air.

An exactly similar experiment, described in many textbooks on hydromechanics, is made as follows. To one end of a straight tube a plane disc is fitted which is capable of sliding on wires projecting from the end of the tube. If the disc is placed at a small distance from the end, and anyone blows steadily into the tube, the disc will be drawn towards the tube instead of being blown off the wires, and will oscillate about a position near the end of the tube.

In the same way we may make a tube by placing two books on a table with their backs parallel and an inch or so apart and laying a sheet of newspaper over them. If anyone blows steadily through the tube so formed, the paper will be sucked in instead of being blown out. 
The following experiment is explicable by the same argument. On the top of a vertical axis balance a thin horizontal rod. At each end of this rod fasten a small vertical square or sail of thin cardboard-the two sails being in the same plane. If anyone blows close to one of these squares and in a direction parallel to its plane, the square will move towards the side on which one is blowing, and the rod with the two sails will rotate about the axis.

The experiments above described can be performed so as to illustrate Hauksbee's Law ; but unless care is taken other causes will be also introduced which affect the phenomena: it is however unnecessary for my purpose to go into these details.

Cut on a Tennis-Ball. Racquet and court-tennis players know that if a strong cut is given to a ball it can be made to rebound off a vertical wall and then (without striking the floor or any other wall) return and hit the wall again. This affords another illustration of Hauksbee's Law. The effect had been noticed by Isaac Newton, who, in his letter to Oldenburg, February, 1672, N.S., says "I remembered that I had often seen "a tennis-ball struck with an oblique racket describe such "a curve line. For, a circular as well as a progressive motion "being communicated to it by that stroke, its parts on that side "where the motions conspire, must press and beat the contiguous "air more violently than on the other; and there excite a "reluctancy and re-action of the air proportionably greater. "And...globular bodies," thus acquiring " a circulating motion... "ought to feel the greater resistance from the ambient aether "on that side where the motions conspire, and thence be con"tinually bowed to the other."

The question was discussed by Magnus in 1837 and by Tait in various papers from 1887 to 1896 . The explanation* is that the cut causes the ball to rotate rapidly about an axis through

* See Magnus on 'Die Abweichung der Geschosse' in the Abhandlungen der Akademie der Wissenschaften, Berlin, 1852, pp. 1-23; Lord Rayleigh, 'On the irregular fight of a tennis ball,' Messenger of Mathematics, Cambridge, 1878, vol. vII, pp. 14-16; and P. G. Tait, Transactions Royal Society, Edinburgh, vol. IXvII, 1893; or Collected Scientific Papers, Cambridge, vol. Ir, 1900, pp. 356-387, and references therein. 
its centre of figure, and the friction of the surface of the ball on the air produces a sort of whirlpool. This rotation is in addition to its motion of translation. Suppose the ball to be spherical and rotating about an axis through its centre perpendicular to the plane of the paper in the direction of the arrow-head, and at the same time moving through still air from left to right parallel to $P Q$. Any motion of the ball perpendicular to $P Q$ will be produced by the pressure of the air on the surface of the ball, and this pressure will, by Hauksbee's Law, be greatest where the velocity of the air relative to the ball is least, and vice versa. To find the velocity of the air relative to the ball we may reduce the centre of the ball to rest, and suppose a stream of air to impinge on the surface of the ball moving with a velocity equal and opposite to that of the centre of the ball.

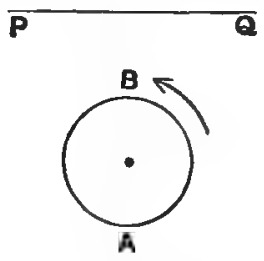

The air is not frictionless, and therefore the air in contact with the surface of the ball will be set in motion by the rotation of the ball and will form a sort of whirlpool rotating in the direction of the arrow-head in the figure. To find the actual velocity of this air relative to the ball we must consider how the motion due to the whirlpool is affected by the motion of the stream of air parallel to $Q P$. The air at $A$ in the whirlpool is moving against the stream of air there, and therefore its velocity is retarded: the air at $B$ in the whirlpool is moving in the same direction as the stream of air there, and therefore its velocity is increased. Hence the relative velocity of the air at $A$ is less than at $B$, and, since the pressure of the air is greatest where the velocity is least, the pressure of the air on the surface of the ball at $A$ is greater than on that at $B$. Hence the ball is forced by this pressure in the direction from the line $P Q$, which we may 
suppose to represent the section of the vertical wall in a racquetcourt. In other words, the ball tends to move at right angles to the line in which its centre is moving and in the direction in which the surface of the front of the ball is being carried by the rotation. Sir J. J. Thomson has pointed out that if we consider the direction in which the nose (or foremost point) of the ball is travelling, we may sum up the results by saying that the ball always follows its nose. Lord Rayleigh has shown that the line of action of resulting force on the ball is perpendicular to the plane containing the direction $(m)$ of motion of the centre of the ball and the axis $(s)$ of spin, and its magnitude varies directly as the velocity of translation, the velocity of spin, and the sine of the angle between the lines $m$ and $s$.

In the case of a lawn tennis-ball, the shape of the ball is altered by a strong cut, and this introduces additional complications.

Spin on a Cricket-Ball. The curl of a cricket-ball in its flight through the air, caused by a spin given by the bowler in delivering the ball, is explained by the same reasoning.

Thus suppose the ball is delivered in a direction lying in a vertical plane containing the middle stumps of the two wickets. A spin round a horizontal axis parallel to the crease in a direction which the bowler's umpire would describe as positive, namely, counter clock-wise, will, in consequence of the friction of the air, cause it to drop, and therefore decrease the length of the pitch. A spin in the opposite direction will cause it to rise, and therefore lengthen the pitch. A spin round a vertical axis in the positive direction, as viewed from above, will make it curl sideways in the air to the left, that is, from leg to off. A spin in the opposite direction will make it curl to the right. A spin given to the ball round the direction of motion of the centre of the ball will not sensibly affect the motion through the air, though it would cause the ball, on hitting the ground, to break. Of course these various kinds of spin can be combined.

Flight of Golf-Balls. The same argument explains the effect of the spin given to a golf-ball by impact with the club. Here the motion takes place for a longer interval of time, and 
additional complications are introduced by the fact that the velocities of translation and rotation are retarded at different rates and that usually there will be some wind blowing in a cross direction. But generally we may say that the effect of the under-cut given by a normal stroke is to cause the ball to rise, and therefore to lengthen the carry. Also, if a wind is blowing across the line to the hole from right to left, a drive, if the player desires a long carry and has sufficient command over his club, should be pulled; but an approach shot, if it is desired that the ball should fall dead, should be sliced because then the ball as soon as it meets the wind will tend to fall dead. Conversely, if the wind is blowing across the course from left to right, the drive should be sliced if a long carry is desired, and an approach shot should be pulled if it is desired that the ball should fall dead.

The questions involving the application of Hauksbee's Law are easy as compared with many of the problems in fluid motion. The analysis required to attack most of these problems is beyond the scope of this book, but one of them may be worth mentioning even though no explanation is given.

The Theory of the Flight of Birds. A mechanical problem of great interest is the explanation of the means by which birds are enabled to fly for considerable distances with no (perceptible) motion of the wings. Albatrosses, to take an instance of special difficulty, have been known to follow for some days ships sailing at the rate of nine or ten knots, and sometimes for considerable periods there is no motion of the wings or body which can be detected, while even if the bird moved its wings it is not easy to understand how it has the muscular energy to propel itself so rapidly and for such a length of time. Of this phenomenon various explanations* have been suggested. Notable among these are Sir Hiram Maxim's of upward aircurrents, Lord Rayleigh's of variations of the wind velocity at different heights above the ground, Dr S. P. Langley's of the

* See G. H. Bryan in the Transactions of the British Association for 1896, vol. Levt, pp. $726-728$. 
incessant occurrence of gusts of wind separated by lulls, and Dr Bryan's of vortices in the atmosphere.

It now seems reasonably certain that the second and third of these sources of energy account for at least a portion of the observed phenomena. The effect of the third cause may be partially explained by noting that the centre of gravity of the bird with extended wings is slightly below the aeroplane or wing surface, so that the animal forms a sort of parachute. The effect of a sudden gust of wind upon such a body is that the aeroplane is set in motion more rapidly than the suspended mass, causing the structure to heel over so as to receive the wind on the under surface of the aeroplane, and this lifts the suspended mass giving it an upward velocity. When the wind falls the greater inertia of the mass carries it on upwards causing the aeroplane to again present its under side to the air; and if while the parachute is in this position the wind is still blowing from the side, the suspended mass is again lifted. Thus the more the bird is blown about, the more it rises in the air; actually birds in flight are carried up by a sudden side gust of wind as we should expect from this theory.

The fact that the bird is in motion tends also to keep it up, for it has been recently shown that a horizontal plane under the action of gravity falls to the ground more slowly if it is travelling through the air with horizontal velocity than it would do if allowed to fall vertically, hence the bird's forward motion causes it to fall through a smaller height between successive gusts of wind than it would do if it were at rest. Moreover it has been proved experimentally that the horse-power required to support a body in horizontal flight by means of an aeroplane is less for high than for low speeds: hence when a side-wind (that is, a wind at right angles to the bird's course) strikes the bird, the lift is increased in consequence of the bird's forward velocity.

Curiosa Physica. When I was writing the first edition of these Recreations, I put together a chapter, following this one, on "Some Physical Questions," dealing with problems such as, in the Theory of Sound, the explanation of the fact that in 
some of Captain Parry's experiments the report of a cannon, when fired, travelled so much more rapidly than the sound of the human voice that observers heard the report of the cannon when fired before that of the order to fire it* : in the Kinetic Theory of Gases, the complications in our universe that might be produced by "Maxwell's demon" $\uparrow$ : in the Theory of Optics, the explanation of the Japanese "magic mirrors," + which reflect the pattern on the back of the mirror, on which the light does not fall; various physiological paradoxes; and the theory of the "spectrum top," by means of which a white surface, on which some black lines are drawn, can be moved so as to give the impression $\S$ that the lines are coloured (red, green, blue, slate, or drab), while the colours change with the direction of rotation: on wave motion it has also been recently shown that if two trains of waves, whose lengths are in the ratio $m-1: m+1$, be superposed, then every $m$ th wave in the system will be bigthus the current opinion that every ninth wave in the open sea is bigger than the other waves may receive scientific confirmation. There is no lack of interesting and curious phenomena in physics, and in some branches, notably in electricity and magnetism, the difficulty is rather one of selection, but I felt that the connection with mathematics was in general either too remote or too technical to justify the insertion of such a collection in a work on elementary mathematical recreations, and therefore I struck out the chapter. I mention the fact now partly to express the hope that some physicist will one day give us a collection of the kind, partly to suggest these questions to those who are interested in such matters.

- The fact is well authenticated. Mr Earnshaw (Philosophical Transactions, Iondon, 1860, pp. 133-148) explained it by the acceleration of a wave caused by the formation of a kind of bore, a view aceepted by Clerk Maxwell and most physicists, bat Six George Airy thought that the explanation was to be found in physiology; see Airy's Sound, second edition, London, 1871, pp. 141, 142.

+ See Theory of Heat, by J. Clerk Maxwell, second edition, London, 1872, p. 308 .

‡ See a memoir by W. E. Ayrton and J. Perry, Proceedings of the Royal Society of London, part I, 1879, vol. xxvin, pp. 127-148.

8 See letters from Mr C. E. Benham and others in Nature, 1894-5; and a paper read by Prof. G. D. Liveing before the Cambridge Philosophical Society, November 26, 1894. 


\section{CHAPTER VI.}

\section{CHESS-BOARD RECREATTONS.}

A chess-board and chess-men lend themselves to recreations many of which are geometrical. The problems are, however, of a distinct type, and sufficiently numerous to deserve a chapter to themselves. A few problems which might be included in this chapter have been already considered in chapter IV.

The ordinary chess-board consists of 64 small squares, known as cells, arranged as shown below in 8 rows and 8 columns. Usually the cells are coloured alternately white and black, or white and red. The cells may be defined by the numbers 11, 12, \&c., where the first digit denotes the number of the column,

\begin{tabular}{|l|l|l|l|l|l|l|l|}
\hline 18 & 28 & 38 & 48 & 58 & 68 & 78 & 88 \\
\hline 17 & 27 & 37 & 47 & 57 & 67 & 77 & 87 \\
\hline 16 & 26 & 36 & 46 & 56 & 66 & 76 & 86 \\
\hline 15 & 25 & 35 & 45 & 55 & 65 & 75 & 85 \\
\hline 14 & 24 & 34 & 44 & 54 & 64 & 74 & 84 \\
\hline 13 & 23 & 33 & 43 & 53 & 63 & 73 & 83 \\
\hline 12 & 22 & 32 & 42 & 52 & 62 & 72 & 82 \\
\hline 11 & 21 & 31 & 41 & 51 & 61 & 71 & 81 \\
\hline
\end{tabular}

and the second digit the number of the row-the two digits representing respectively the abscissa and ordinate of the midpoints of the cells. I use this notation in the following pages. A generalized board consists of $n^{2}$ cells arranged in $n$ rows and 
$n$ columns. Most of the problems which I shall describe can be extended to meet the case of a board of $n^{2}$ cells.

The usual chess-pieces are Kings, Queens, Bishops, Knights, and Rooks or Castles; there are also Pawns. I assume that the moves of these pieces are known to the reader.

With the game itself and with chess problems of the usual type I do not concern myself. Particular positions of the pieces may be subject to mathematical analysis, but in general the moves open to a player are so numerous as to make it impossible to see far ahead. Probably this is obvious, but it may emphasize how impossible it is to discuss the theory of the game effectively if $\mathbf{I}$ add that it has been shown that there may be as many as 197299 ways of playing the first four moves, and nearly 72000 different positions at the end of the first four moves (two on each side), of which 16556 arise when the players move pawns only*.

Relative Value of Pieces. The first question to which I will address myself is the determination of the relative values of the different chess-piecest.

If a piece is placed on a cell, the number of cells it commands depends in general on its position. We may estimate the value of the piece by the average number of cells which it commands when placed in succession on every cell of the board. This is equivalent to saying that the value of a piece may be estimated by the chance that if it and a king are put at random on the board, the king will be in check: if no other restriction is imposed this is called a simple check. On whatever cell the piece is originally placed there will remain 63 other cells on which the king may be placed. It is equally probable that it may be put on any one of them. Hence the chance that it will be in check is $1 / 63$ of the average number of cells commanded by the piece.

* L'Intermédiaire des Mathématiciens, Paris, December, 1903, vol. $\mathbf{z}, \mathrm{pp}$. 305-308: also Royal Engineers Journal, London, August-November, 1889; or British Association Transactions, 1890, p. 745 .

† H. M. Taylor, Philosophical Magazine, Maroh, 1876, series 5, vol. I, pp. $221-229$. 
A rook put on any cell commands 14 other cells. Wherever the rook is placed there will remain 63 cells on which the king may be placed, and on which it is equally likely that it will be placed. Hence the chance of a simple check is $14 / 63$, that is, 2/9. Similarly on a board of $n^{2}$ cells the chance is $2(n-1) /\left(n^{2}-1\right)$, that is, $2 /(n+1)$.

A knight when placed on any of the 4 corner cells like 11 commands 2 cells. When placed on any of the 8 cells like 12 and 21 it commands 3 cells. When placed on any of the 4 cells like 22 or any of the 16 boundary cells like $13,14,15$, 16 , it commands 4 cells. When placed on any of the 16 cells like 23, 24, 25, 26, it commands 6 cells. And when placed on any of the remaining 16 middle cells it commands 8 cells. Hence the average number of cells commanded by a knight put on a chess-board is $(4 \times 2+8 \times 3+20 \times 4+16 \times 6+16 \times 8) / 64$, that is, 336/64. Accordingly if a king and a knight are put on the board, the chance that the king will be in simple check is $336 / 64 \times 63$, that is $1 / 12$. Similarly on a board of $n^{2}$ cells the chance is $8(n-2) / n^{2}(n+1)$.

A bishop when placed on any of the ring of 28 boundary cells commands 7 cells. When placed on any ring of the 20 cells next to the boundary cells, it commands 9 cells. When placed on any of the 12 cells forming the next ring, it commands 11 cells. When placed on the 4 middle cells it commands 13 cells. Hence, if a king and a bishop are put on the board the chance that the king will be in simple check is $(28 \times 7+20 \times 9+12 \times 11+4 \times 13) / 64 \times 63$, that is, $5 / 36$. Similarly on a board of $n^{2}$ cells, when $n$ is even, the chance is $2(2 n-1) / 3 n(n+1)$. When $n$ is odd the analysis is longer, owing to the fact that in this case the number of white cells on the board differs from the number of black cells. I do not give the work, which presents no special difficulty.

A queen when placed on any cell of a board commands all the cells which a bishop and a rook when placed on that cell would do. Hence, if a king and a queen are put on the board, the chance that the king will be in simple check is $2 / 9+5 / 36$, that is, $13 / 36$. Similarly on a board of $n^{2}$ cells, when $n$ is even, the chance is $2(5 n-1) / 3 n(n+1)$, 
On the above assumptions the relative values of the rook, knight, bishop, and queen are 16, 6, 10, 26. According to Staunton's Chess-Player's Handbook the actual values, estimated empirically, are in the ratio of 548, 305, 350, 994; according to Von Bilguer the ratios are 540, 350, 360, 1000-the value of a pawn being taken as 100 .

There is considerable discrepancy between the above results as given by theory and practice. It has been, however, suggested that a better test of the value of a piece would be the chance that when it and a king were put at random on the board it would check the king without giving the king the opportunity of taking it. This is called a safe check as distinguished from a simple check.

Applying the same method as above, the chances of a safe check work out as follows. For a rook the chance of a safe check is $(4 \times 12+24 \times 11+36 \times 10) / 64 \times 63$, that is, $1 / 6$; or on a board of $n^{2}$ cells is $2(n-2) / n(n+1)$. For a knight all checks are safe, and therefore the chance of a safe check is $1 / 12$; or on a board of $n^{2}$ cells is $8(n-2) / n^{2}(n+1)$. For a bishop the chance of a safe check is $364 / 64 \times 63$, that is, $13 / 144$; or on a board of $n^{2}$ cells, when $n$ is even, is $2(n-2)(2 n-3) / 3 n^{2}(n+1)$. For a queen the chance of a safe check is $1036 / 64 \times 63$, that is, $37 / 144$; or on a board of $n^{2}$ cells, is $2(n-2)(5 n-3) / 3 n^{2}(n+1)$, when $n$ is even.

On this view the relative values of the rook, knight, bishop, queen are 24, 12, 13, 37; while, according to Staunton, experience shows that they are approximately 22, 12, 14, 40, and according to Von Bilguer, 18, 12, 12, 33.

The same method can be applied to compare the values of combinations of pieces. For instance the value of two bishops (one restricted to white cells and the other to black cells) and two rooks, estimated by the chance of a simple check, are respectively $35 / 124$ and $37 / 93$. Hence on this view a queen in general should be more valuable than two bishops but less valuable than two rooks. This agrees with experience.

An analogous problem consists in finding the chance that two kings, put at random on the board, will not occupy adjoining cells, that is, that neither would (were such a move possible) 
check the other. The chance is $43 / 48$, and therefore the chance that they will occupy adjoining cells is $5 / 48$. If three kings are put on the board, the chance that no two of them occupy adjoining cells is 1061/1488. The corresponding chances* for a board of $n^{2}$ cells are $(n-1)(n-2)\left(n^{2}+3 n-2\right) / n^{2}\left(n^{3}-1\right)$ and $(n-1)(n-2)\left(n^{4}+3 n^{8}-20 n^{2}-30 n+132\right) / n^{2}\left(n^{2}-1\right)\left(n^{2}-2\right)$.

The Eight Queens Problem $\uparrow$. One of the classical problems connected with a chess-board is the determination of the number of ways in which eight queens can be placed on a chess-board-or more generally, in which $n$ queens can be placed on a board of $n^{2}$ cells-so that no queen can take any other. This was proposed originally by Franz Nauck in 1850 .

In $1874 \mathrm{Dr} \mathrm{S}$. Günther+ suggested a method of solution by means of determinants. For, if each symbol represents the corresponding cell of the board, the possible solutions for a board of $n^{2}$ cells are given by those terms, if any, of the determinant

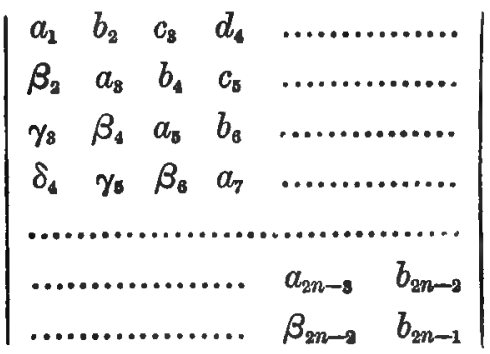

in which no letter and no suffix appears more than once.

The reason is obvious. Every term in a determinant contains one and only one element out of every row and out of every column: hence any term will indicate a position on the board in which the queens cannot take one another by moves rook-wise. Again in the above determinant the letters and suffixes are so arranged that all the same letters and all the

* L'Intermédiaire des Mathématiciens, Paris, 1897, vol. Iv, p. 6, and 1901, vol. viII, p. 140.

+ On the history of this problem see W. Ahrens, Mathematische Unterhaltungen und Spiele, Leipzig, 1901, chap. ix.

‡ Grunert's Archiv der Mathematik und Physit, 1874, vol. LvI, pp. 281-292. 
same suffixes lie along bishop's paths : hence, if we retain only those terms in each of which all the letters and all the suffixes are different, they will denote positions in which the queens cannot take one another by moves bishop-wise. It is clear that the signs of the terms are immaterial.

In the case of an ordinary chess-board the determinant is of the 8 th order, and therefore contains 8 !, that is, 40320 terms, so that it would be out of the question to use this method for the usual chess-board of 64 cells or for a board of larger size unless some way of picking out the required terms could be discovered.

A way of effecting this was suggested by Dr J. W.L. Glaisher* in 1874, and so far as I am aware the theory remains as he left it. He showed that if all the solutions of $n$ queens on a board of $n^{2}$ cells were known, then all the solutions of a certain type for $n+1$ queens on a board of $(n+1)^{2}$ cells could be deduced, and that all the other solutions of $n+1$ queens on a board of $(n+1)^{2}$ cells could be obtained without difficulty. The method will be sufficiently illustrated by one instance of its application.

It is easily seen that there are no solutions when $n=2$ and $n=3$. If $n=4$ there are two terms in the determinant which give solutions, namely, $b_{2} c_{6} \gamma_{8} \beta_{6}$ and $c_{8} \beta_{2} b_{6} \gamma_{6}$. To find the solutions when $n=5$, Glaisher proceeded thus. In this case, Günther's determinant is

$$
\left|\begin{array}{lllll}
a_{1} & b_{2} & c_{8} & d_{4} & e_{6} \\
\beta_{2} & a_{8} & b_{6} & c_{5} & d_{6} \\
\gamma_{8} & \beta_{4} & a_{5} & b_{6} & c_{7} \\
\delta_{4} & \gamma_{5} & \beta_{6} & a_{7} & b_{8} \\
\epsilon_{6} & \delta_{6} & \gamma_{7} & \beta_{8} & a_{9}
\end{array}\right|
$$

To obtain those solutions (if any) which involve $a_{9}$ it is sufficient to append $a_{9}$ to such of the solutions for a board of 16 cells as do not involve $a$. As neither of those given above involves an $a$ we thus get two solutions, namely, $b_{2} c_{6} \gamma_{8} \beta_{8} a_{9}$ and $c_{3} \beta_{2} b_{6} \gamma_{8} a_{9}$.

* Philosophical Magazine, London, December, 1874, series 4, rol. xuvII, pp. $457-467$. 
The solutions which involve $a_{1}, e_{5}$ and $\epsilon_{5}$ can be written down by symmetry. The eight solutions thus obtained are all distinct; we may call them of the first type.

The above are the only solutions which can involve elements in the corner squares of the determinant. Hence the remaining solutions are obtainable from the determinant

$$
\left|\begin{array}{ccccc}
0 & b_{2} & c_{3} & d_{4} & 0 \\
\beta_{2} & a_{8} & b_{4} & c_{8} & d_{6} \\
\gamma_{8} & \beta_{4} & a_{6} & b_{6} & c_{7} \\
\delta_{4} & \gamma_{8} & \beta_{6} & a_{7} & b_{8} \\
0 & \delta_{8} & \gamma_{7} & \beta_{8} & 0
\end{array}\right|
$$

If, in this, we take the minor of $b_{2}$ and in it replace by zero every term involving the letter $b$ or the suffix 2 we shall get all solutions involving $b_{9}$. But in this case the minor at once reduces to $d_{6} a_{5} \delta_{4} \beta_{8}$. We thus get one solution, namely, $b_{2} d_{6} a_{5} \delta_{4} \beta_{8}$. The solutions which involve $\beta_{2}, \delta_{4}, \delta_{8}, \beta_{8}, b_{8}, d_{6}$, and $d_{4}$ can be obtained by symmetry. Of these eight solutions it is easily seen that only two are distinct: these may be called solutions of the second type.

Similarly the remaining solutions must be obtained from the determinant

$$
\left|\begin{array}{ccccc}
0 & 0 & c_{3} & 0 & 0 \\
0 & a_{8} & b_{4} & c_{8} & 0 \\
\gamma_{8} & \beta_{4} & a_{8} & b_{8} & c_{7} \\
0 & \gamma_{8} & \beta_{8} & a_{7} & 0 \\
0 & 0 & \gamma_{7} & 0 & 0
\end{array}\right|
$$

If, in this, we take the minor of $c_{B}$, and in it replace by zero every term involving the letter $c$ or the suffix 3 , we shall get all the solutions which involve $c_{s}$. But in this case the minor vanishes. Hence there is no solution involving $c_{3}$, and therefore by symmetry no solutions which involve $\gamma_{B}, \gamma_{r}$, or $c_{7}$. Had there been any solutions involving the third element in the first or last row or column of the determinant we should have described them as of the third type. 
Thus in all there are ten and only ten solutions, namely, eight of the first type, two of the second type, and none of the third type.

Similarly, if $n=6$, we obtain no solutions of the first type, four solutions of the second type, and no solutions of the third type; that is, four solutions in all. If $n=7$, we obtain sixteen solutions of the first type, twenty-four solutions of the second type, no solutions of the third type, and no solutions of the fourth type; that is, forty solutions in all. If $n=8$, we obtain sixteen solutions of the first type, fifty-six solutions of the second type, and twenty solutions of the third type, that is, ninety-two solutions in all.

It will be noticed that all the solutions of one type are not always distinct. In general, from any solution seven others can be obtained at once. Of these eight solutions, four consist of the initial or fundamental solution and the three similar one: obtained by turning the board through one, two, or three right angles; the other four are the reflexions of these in a mirror. but in any particular case it may happen that the reflexions reproduce the originals, or that a rotation through one or two right angles makes no difference. Thus on boards of $4^{2}, 5^{2}, 6^{2}$, $7^{2}, 8^{2}, 9^{2}, 10^{2}$ cells there are respectively $1,2,1,6,12,46,92$ fundamental solutions; while altogether there are respectively $2,10,4,40,92,352,724$ solutions.

The following collection of fundamental solutions may interest the reader. Each position on the board of the queens is indicated by a number, but as necessarily one queen is on each column I can use a simpler notation than that explained on page 109. In this case the first digit represents the number of the cell occupied by the queen in the first column reckoned from one end of the column, the second digit the number in the second column, and so on. Thus on a board of $4^{2}$ cells the solution 3142 means that one queen is on the $3 r d$ square of the first column, one on the 1st square of the second column, one on the 4th square of the third column, and one on the 2nd square of the fourth column. If a fundamental solution gives rise to only four solutions the number which indicates it is placed 
in curved brackets, ( ); if it gives rise to only two solutions the number which indicates it is placed in square brackets, [ ]; the other fundamental solutions give rise to eight solutions each.

On a board of $4^{2}$ cells there is 1 fundamental solution: namely, [3142].

On a board of $5^{2}$ cells there are 2 fundamental solutions: namely, 14253, [25314]. It may be noted that the cyclic solutions 14253, 25314, 31425, 42531, 53142 give five superposable arrangements by which five white queens, five black queens, five red queens, five yellow queens, and five blue queens can be put simultaneously on the board so that no queen can be taken by any other queen of the same colour.

On a board of $6^{2}$ cells there is 1 fundamental solution: namely, (246135). The four solutions are superposable. The puzzle for this case is sold in the streets of London for a penny, a small wooden board being ruled in the manner shown in the diagram and having holes drilled in it at the points marked by dots. The object is to put six pins into the holes so that no two are connected by a straight line.

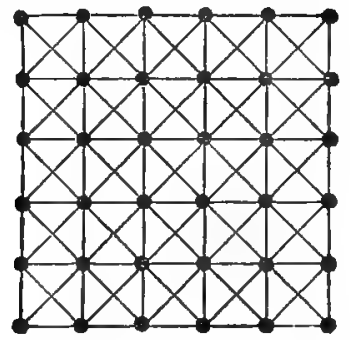

On a board of $7^{2}$ cells there are 6 fundamental solutions: namely, 1357246, 3572461, (5724613), 4613572, 3162574, (2574136). It may be noted that the solution 1357246 gives by cyclic permutations seven superposable arrangements.

On a board of $8^{2}$ cells there are 12 fundamental solutions: namely, 25713864, 57138642, 71386425, 82417536, 68241753, $36824175,64713528,36814752$, 36815724, 72418536, 26831.475, (64718253). The arrangement in this order is due to Mr Oram. It will be noticed that the 10th, 11th, and 12th solutions somewhat resemble the 4 th, 6 th, and 7 th respectively. The 6 th 
solution is the only one in which no three queens are in a straight line. It is impossible * to find eight superposable solutions; but we can in five typical ways pick out six solutions which can be superposed, and to some of these it is possible to add 2 sets of 7 queens, thus filling 62 out of the 64 cells with 6 sets of 8 queens and 2 sets of 7 queens, no one of which can take another of the same set. Here is such a solution: 16837425, 27368514, 35714286, 41586372, 52473861, 68241753, 73625140, 04152637. Similar superposition problems can be framed for boards of other sizes.

For any reader who wishes to go further, I may add that it has been shown that on a board of $n^{2}$ cells, there are 46 fundamental solutions when $n=9$, there are 92 when $n=10$, there are 341 when $n=11$, there are 1766 when $n=12$, and there are 1346 when $n=13$.

On any board empirical solutions may be found with but little difficulty, and Mr Derrington has constructed the following table of solutions:

2.4.1.3

2.4 .1 .3 .5

2.4 .6 .1 .3 .5

2.4 .6 .1 .3 .5 .7

$2.4 .6 \cdot 8 \cdot 3 \cdot 1 \cdot 7 \cdot 5$

$2 \cdot 4 \cdot 1 \cdot 7 \cdot 9 \cdot 6 \cdot 3 \cdot 5 \cdot 8$

$2.4 .6 .8 \cdot 10 \cdot 1 \cdot 3 \cdot 5 \cdot 7 \cdot 9$

$2 \cdot 4 \cdot 6 \cdot 8 \cdot 10 \cdot 1 \cdot 3 \cdot 5 \cdot 7 \cdot 9 \cdot 11$

2.4.6.8.10.12.1.3.5.7.9.11

$2.4 .6 .8 \cdot 10 \cdot 12.1 .3 .5 \cdot 7 \cdot 9.11 .13$

9.7.5.3.1.13.11.6.4.2.14.12.10.8

$15 \cdot 9 \cdot 7.5 \cdot 3 \cdot 1 \cdot 13 \cdot 11 \cdot 6 \cdot 4 \cdot 2 \cdot 14.12 \cdot 10.8$

$2.4 \cdot 6 \cdot 8 \cdot 10 \cdot 12.14 \cdot 16 \cdot 1 \cdot 3 \cdot 5 \cdot 7 \cdot 9 \cdot 11.13 .15$

$2.4 \cdot 6 \cdot 8 \cdot 10.12 \cdot 14 \cdot 16 \cdot 1 \cdot 3 \cdot 5 \cdot 7 \cdot 9 \cdot 11.13 .15 .17$

2.4.6.8.10.12.14.16.18.1.3.5.7.9.11.13.15.17

2.4.6.8.10.12.14.16.18.1.3.5.7.9.11.13.15.17.19

$12.10 .8 \cdot 6 \cdot 4.2 \cdot 20.18 \cdot 16 \cdot 14 \cdot 9 \cdot 7 \cdot 5 \cdot 3.1 .19 .17 .15 .13 .11$ 21. $12.10 .8 \cdot 6 \cdot 4 \cdot 2 \cdot 20 \cdot 18 \cdot 16 \cdot 14 \cdot 9 \cdot 7 \cdot 5 \cdot 3 \cdot 1 \cdot 19 \cdot 17 \cdot 15 \cdot 13.11$, for a board of $4^{2}$ cells

$5^{2}$,

$6^{2}$,

$7^{2}$

$8^{2}$,

$9^{2}$

$10^{2}$

$11^{2}$,

$12^{2}$

$13^{2}$

$14^{2}$

$15^{2}$

$16^{2}$

$17^{2}$,

$18^{2}$

$19^{2}$

$20^{2}$

218

* See G. T. Bennett, The Messenger of Mathematics, Cambridge, June, 1909, vol. exxix, pp. 19-21. 
and so on. The rule is obvious except when $n$ is of the form $6 m+2$ or $6 m+3$.

Maximum Pieces Problem*. The Eight Queens Problem suggests the somewhat analogous question of finding the maximum number of kings-or more generally of pieces of one type-which can be put on a board so that no one can take any other, and the number of solutions possible in each case.

In the case of kings the number is 16; for instance, one solution is when they are put on the cells $11,13,15,17,31,33$, $35,37,51,53,55,57,71,73,75,77$. For queens, it is obvious that the problem is covered by the analysis already given, and the number is 8 . For bishops the number is 14, the pieces being put on the boundary cells; for instance one solution is when they are put on the cells $11,12,13,14,15,16,17,81,82$, $83,84,85,86,87$, there are 256 solutions. For knights the number is 32 ; for instance, they can be put on all the white or on all the black cells, and there are 2 fundamental solutions. For rooks it is obvious that the number is 8 , and there are in all 8 ! solutions.

Minimum Pieces Problem*. Another problem of a somewhat similar character is the determination of the minimum number of kings-or more generally of pieces of one typewhich can be put on a board so as to command or occupy all the cells.

For kings the number is 9 ; for instance, they can be put on the cells 11, 14, 17, 41,44, 47, 71, 74, 77. For queens the number is 5 ; for instance, they can be put on the cells 18,35 , $41,76,82$. For bishops the number is 8; for instance, they can be put on the cells $41,42,43,44,45,46,47,48$. For knights the number is 12 ; for instance, they can be put on the cells $26,32,33,35,36,43,56,63,64,66,67$, and 73 -constituting four triplets arranged symmetrically. For rooks the number is 8 , and the solutions are obvious.

- Mr H. F. Dudeney has written on these problems in the Weekly Dispatch. 
For queens the problem has been also discussed for a board of $n^{2}$ cells where $n$ has various values*. One queen can be placed so as to command all the cells when $n=2$ or 3 , and there is only 1 fundamental solution. Two queens are required when $n=4$; and there are 3 fundamental solutions, namely, when they are placed on the cells 11 and 33 , or on the cells 12 and 42 , or on the cells 22, 23: these give 12 solutions in all. Three queens are required when $n=5$; and there are 37 fundamental solutions, giving 186 solutions in all. Three queens are also required when $n=6$, but there is only 1 fundamental solution, namely, when they are put on the cells 11, 35, and 53, giving 4, solutions in all. Four queens are required when $n=7$, one solution is when they are put on the cells 12,26 , 41,55 .

Jaenisch proposed also the problem of the determination of the minimum number of queens which can be placed on a board of $n^{2}$ cells so as to command all the unoccupied cells, subject to the restriction that no queen shall attack the cell occupied by any other queen. In this case three queens are required when $n=4$, for instance, they can be put on the cells 11, 23, 42; and there are 2 fundamental solutions, giving 16 solutions in all. Three queens are required when $n=5$, for instance, they can be put on the cells 11, 24, 43, or on the cells 11,34, 53; and there are 2 fundamental solutions in all. Four queens are required when $n=6$, for instance, when they are put on the cells 13,36 , 41, 64; and there are 17 fundamental solutions. Four queens are required when $n=7$, and there is only 1 fundamental solution, namely, that already mentioned, when they are put on the cells 12, 26, 41, 55, which gives 8 solutions in all. Five queens are required when $n=8$, and there are no less than 91 fundamental solutions; for instance, one is when they are put on the cells $11,23,37,62,76$.

I leave to any of my readers who may be interested in such questions the discussion of the corresponding problems for the

* C. F. de Jaenisch, Applications de l'Analyse Mathématique au Jeu des Échecs, Petrograd, 1862, Appendix, p. 244 et seq.; see also L'Intermédiaire des Mathématiciens, Paris, 1901, vol. vmI, p. 88. 
other pieces*, and of the number of possible solutions in each case.

A problem of the same nature would be the determination of the minimum number of queens (or other pieces) which can be placed on a board so as to protect one another and command all the unoccupied cells. For queens the number is 5 ; for instance, they can be put on the cells $24,34,44$, 54 and 84. For bishops the number is 10; for instance, they can be put on the cells $24,25,34,35,44,45,64,65,74$, and 75 . For knights the number is 14; for instance, they can be put on the cells $32,33,36,37,43,44,45,46,63,64,65,66,73$, and 76 : the solution is semi-symmetrical. For rooks the number is 8 , and a solution is obvious. I leave to any who are interested in the subject the determination of the number of solutions in each case.

In connexion with this class of problems, I may mention two other questions, to which Captain Turton first called my attention, of a somewhat analogous character.

The first of these is to place eight queens on a chess-board so as to command the fewest possible squares. Thus, if queens are placed on cells $21,22,62,71,73,77,82,87$, eleven cells on the board will not be in check; the same number can be obtained by other arrangements. Is it possible to place the eight queens so as to leave more than eleven cells out of check? I have never succeeded in doing so, nor in showing that it is impossible to do it.

The other problem is to place $m$ queens ( $m$ being less than 5) on a chess-board so as to command as many cells as possible. For instance, four queens can be placed in several ways on the board so as to command 58 cells besides those on which the queens stand, thus leaving only 2 cells which are not commanded; for instance, queens may be placed on the cells 35, 41,

* The problem for knightr was discussed in L'Intermediaire des Mathématiciens, Paris, 1896, vol. IIr, p. 58; 1897, vol. IV, pp. 15-17, 254; 1898, rol. $\nabla$, pp. 87, 230-231. 
76, and 82. Analogous problems with other pieces will suggest themaselves.

There are endless similar questions in which combinations of pieces are involved. For instance, if queens are put on the cells $35,41,76$, and 82 they command or occupy all but two cells, and these two cells may be commanded or occupied by $a$ queen, a king, a rook, a bishop, or a pawn. If queens are put on the cells $22,35,43$, and 54 they command or occupy all but three cells, and two of these three cells may be commanded by a knight which occupies the third of them.

Re-Entrant Paths on a Chess-Board. Another problem connected with the chess-board consists in moving a piece in such a manner that it shall move successively on to every possible cell once and only once.

Knight's Re-Entrant Path. I begin by discussing the classical problem of a knight's tour. The literature* on this subject is so extensive that I make no attempt to give a full account of the various methods for solving the problem, and I shall content myself by putting together a few notes on some of the solutions I have come across, particularly on those due to De Moivre, Euler, Vandermonde, Warnsdorff, and Roget.

On a board containing an even number of cells the path may or may not be re-entrant, but on a board containing an odd number of cells it cannot be re-entrant. For, if a knight begins on a white cell, its first move must take it to a black cell, the next to a white cell, and so on. Hence, if its path passes through all the cells, then on a board of an odd number of cells the last move must leave it on a cell of the same colour as that on which it started, and therefore these cells cannot be connected by one move.

- For a bibliography see A. van der Linde, Geschichte und Literatur des Schachspiels, Berlin, 1874, vol. Ir, pp. 101-111. On the problem and its history gee a memoir by P. Volpicelli in Atti della Reale Accademia dei Lincei, Rome, 1872, vol. xxv, pp. 87-162: also Applications de l'Analyse Mathématique au Jeu des Échecs, by C. F. de Jaenisch, 3 vols., Petrograd, 1862-3 ; and General Parmentier, Association Francaise pour l'avancement des Sciences, 1891, $1892,1894$. 
The earliest solutions of which I have any knowledge are those given at the beginning of the eighteenth century by $\mathrm{De}$ Montmort and De Moivre*. They apply to the ordinary chessboard of 64 cells, and depend on dividing (mentally) the board into an inner square containing sixteen cells surrounded by an outer ring of cells two deep. If initially the knight is placed on a cell in the outer ring, it moves round that ring always in the same direction so as to fill it up completely-only going into the inner square when absolutely necessary. When the outer ring is filled up the order of the moves required for filling the remaining cells presents but little difficulty. If initially the knight is placed on the inner square the process must be reversed. The method can be applied to square and rectangular boards of all sizes. It is illustrated sufficiently by De Moivre's solution which is given below, where the numbers

\begin{tabular}{|c|c|c|c|c|c|c|c|}
\hline 34 & 49 & 22 & 11 & 36 & 39 & 24 & 1 \\
\hline 21 & 10 & 35 & 50 & 23 & 12 & 37 & 40 \\
\hline 48 & 33 & 62 & 57 & 38 & 25 & 2 & 13 \\
\hline 9 & 20 & 51 & 54 & 63 & 60 & 41 & 26 \\
\hline 32 & 47 & 58 & 61 & 56 & 53 & 14 & 3 \\
\hline 19 & 8 & 55 & 52 & 59 & 64 & 27 & 42 \\
\hline 46 & 31 & 6 & 17 & 44 & 29 & 4 & 15 \\
\hline 7 & 18 & 45 & 30 & 5 & 16 & 43 & 28 \\
\hline
\end{tabular}

De Moivre's Solution.

\begin{tabular}{|c|c|c|c|c|c|}
\hline 30 & 21 & 6 & 15 & 28 & 19 \\
\hline 7 & 16 & 29 & 20 & 5 & 14 \\
\hline 22 & 31 & 8 & 35 & 18 & 27 \\
\hline 9 & 36 & 17 & 26 & 13 & 4 \\
\hline 32 & 23 & 2 & 11 & 34 & 25 \\
\hline 1 & 10 & 33 & 24 & 3 & 12 \\
\hline
\end{tabular}

Euler's Thirty-six Cell Solution.

indicate the order in which the cells are occupied successively. I place by its side a somewhat similar re-entrant solution, due to Euler, for a board of 36 cells. If a chess-board is used it is convenient to place a counter on each cell as the knight leaves it.

The earliest serious attempt to deal with the subject by

- They were sent by their authors to Brook Taylor who seems to have previously suggested the problem. I do not know where they were first pub. lished; they were quoted by Ozanam and Montucla, see Ozanam, 1803 edition, vol. I, p. 178 ; 1840 edition, p. 80. 
mathematical analysis was made by Euler* in 1759: it was due to a suggestion made by $\mathrm{L}$. Bertrand of Geneva, who subsequently (in 1778) issued an account of it. This method is applicable to boards of any shape and size, but in general the solutions to which it leads are not symmetrical and their mutual connexion is not apparent.

Euler commenced by moving the knight at random over the board until it has no move open to it. With care this will leave only a few cells not traversed: denote them by $a, b, \ldots$ His method consists in establishing certain rules by which these vacant cells can be interpolated into various parts of the circuit, and by which the circuit can be made re-entrant.

The following example, mentioned by Legendre as one of exceptional difficulty, illustrates the method. Suppose that

\begin{tabular}{|c|c|c|c|c|c|c|c|}
\hline 55 & 58 & 29 & 40 & 27 & 14 & 19 & 22 \\
\hline 60 & 39 & 56 & 43 & 30 & 21 & 26 & 46 \\
\hline 57 & 54 & 59 & 28 & 41 & 18 & 23 & 20 \\
\hline 38 & 61 & 42 & 31 & 8 & 25 & 46 & 17 \\
\hline 53 & 32 & 37 & $a$ & 47 & 16 & 9 & 24 \\
\hline 50 & 3 & 52 & 33 & 36 & 7 & 12 & 15 \\
\hline 1 & 34 & 5 & 48 & $b$ & 14 & $c$ & 10 \\
\hline 4 & 49 & 2 & 35 & 6 & 11 & $d$ & 13 \\
\hline
\end{tabular}

Figure i.

\begin{tabular}{|c|c|c|c|c|c|c|c|}
\hline 22 & 25 & 50 & 39 & 52 & 35 & 60 & 57 \\
\hline 27 & 40 & 23 & 36 & 49 & 58 & 53 & 34 \\
\hline 24 & 21 & 26 & 51 & 38 & 61 & 56 & 59 \\
\hline 41 & 28 & 37 & 48 & 3 & 54 & 33 & 62 \\
\hline 20 & 47 & 42 & 13 & 32 & 63 & 4 & 55 \\
\hline 29 & 16 & 19 & 46 & 43 & 2 & 7 & 10 \\
\hline 18 & 45 & 14 & 31 & 12 & 9 & 64 & 6 \\
\hline 15 & 30 & 17 & 44 & 1 & 6 & 11 & 8 \\
\hline
\end{tabular}

Figure ii.

Example of Euler's Method.

we have formed the route given in figure $\mathrm{i}$ above; namely, 1 , $2,3, \ldots, 59,60$; and that there are four cells left untraversed, namely, $a, b, c, d$.

We begin by making the path 1 to 60 re-entrant. The cell 1 commands a cell $p$, where $p$ is 32,52 , or 2 . The cell 60 commands a cell $q$, where $q$ is 29,59 , or 51 . Then, if any of these values of $p$ and $q$ differ by unity, we can make the route

* Mémoires de Berlin for 1759, Berlin, 1766, pp. 310-337; or Commentationes Arithmeticae Collectae, Petrograd, 1849, vol. r, pp. 337--355. 
re-entrant. This is the case here if $p=52, q=51$. Thus the cells $1,2,3, \ldots, 51 ; 60,59, \ldots, 52$ form a re-entrant route of 60 moves. Hence, if we replace the numbers $60,59, \ldots, 52$ by 52 , $53, \ldots, 60$, the steps will be numbered consecutively. I recommend the reader who wishes to follow the subsequent details of Euler's argument to construct this square on a piece of paper before proceeding further.

Next, we proceed to add the cells $a, b, d$ to this route. In the new diagram of 60 cells formed as above the cell $a$ commands the cells there numbered $51,53,41,25,7,5$, and 3 . It is indifferent which of these we select: suppose we take 51. Then we must make 51 the last cell of the route of 60 cells, so that we can continue with $a, b, d$. Hence, if the reader will add 9 to every number on the diagram he has constructed, and then replace $61,62, \ldots, 69$ by $1,2, \ldots, 9$, he will have a route which starts from the cell occupied originally by 60 , the 60 th move is on to the cell occupied originally by 51 , and the 61 st, $62 \mathrm{nd}$, 63 rd moves will be on the cells $a, b, d$ respectively.

It remains to introduce the cell $c$. Since $c$ commands the cell now numbered 25 , and 63 commands the cell now numbered 24 , this can be effected in the same way as the first route was made re-entrant. In fact, the cells numbered $1,2, \ldots, 24 ; 63$, $62, \ldots, 25, c$ form a knight's path. Hence we must replace $63,62, \ldots, 25$ by the numbers $25,26, \ldots, 63$, and then we can fill up c with 64 . We have now a route which covers the whole board.

Lastly, it remains to make this route re-entrant. First, we must get the cells 1 and 64 near one another. This can be effected thus. Take one of the cells commanded by 1, such as 28, then 28 commands 1 and 27. Hence the cells $64,63, \ldots, 28$; $1,2, \ldots, 27$ form a route; and this will be represented in the diagram if we replace the cells numbered $1,2, \ldots, 27$ by 27 , $26, \ldots, 1$.

The cell now occupied by 1 commands the cells 26, 38, 54, $12,2,14,16,28$; and the cell occupied by 64 commands the cells $13,43,63,55$. The cells 13 and 14 are consecutive, and therefore the cells $64,63, \ldots, 14 ; 1,2, \ldots, 13$ form a route. 
Hence we must replace the numbers $1,2, \ldots, 13$ by $13,12, \ldots$, 1 , and we obtain a re-entrant route covering the whole board, which is represented in the second of the diagrams given above. Euler showed how seven other re-entrant routes can be deduced from any given re-entrant route.

It is not difficult to apply the method so as to form a route which begins on one given cell and ends on any other given cell.

Euler next investigated how his method could be modified so as to allow of the imposition of additional restrictions.

An interesting example of this kind is where the first 32 moves are confined to one-half of the board. One solution of this is delineated below. The order of the first 32 moves

\begin{tabular}{|c|c|c|c|c|c|c|c|}
\hline 58 & 43 & 60 & 37 & 52 & 41 & 62 & 35 \\
\hline 49 & 46 & 67 & 42 & 61 & 86 & 53 & 40 \\
\hline 44 & 59 & 48 & 51 & 38 & 55 & 34 & 63 \\
\hline 47 & 50 & 45 & 56 & 33 & 64 & 39 & 54 \\
\hline 23 & 7 & 32 & 1 & 24 & 13 & 18 & 15 \\
\hline 31 & 2 & 23 & 6 & 19 & 16 & 27 & 12 \\
\hline 8 & 21 & 4 & 29 & 10 & 25 & 14 & 17 \\
\hline 3 & 30 & 9 & 20 & 5 & 28 & 11 & 26 \\
\hline
\end{tabular}

Euler's Half-board Solution.

\begin{tabular}{|c|c|c|c|c|c|c|c|}
\hline 60 & 45 & 62 & 41 & 60 & 89 & 54 & 85 \\
\hline 63 & 48 & 51 & 48 & 53 & 36 & 57 & 88 \\
\hline 46 & 49 & 44 & 61 & 40 & 59 & 34 & 65 \\
\hline 43 & 64 & 47 & 52 & 33 & 56 & 37 & 58 \\
\hline 26 & 5 & 24 & 1 & 20 & 15 & 32 & 11 \\
\hline 23 & 2 & 27 & 8 & 29 & 19 & 17 & 14 \\
\hline 6 & 25 & 4 & 21 & 16 & 10 & 20 & 81 \\
\hline 3 & 22 & 7 & 28 & 9 & 30 & 13 & 18 \\
\hline
\end{tabular}

Roget's Half-board Solution.

can be determined by Euler's method. It is obvious that, if to the number of each such move we add 32 , we shall have a corresponding set of moves from 33 to 64 which would cover the other half of the board; but in general the cell numbered 33 will not be a knight's move from that numbered 32, nor will 64 be a knight's move from 1 .

Euler however proceeded to show how the first 32 moves might be determined so that, if the half of the board containing the corresponding moves from 33 to 64 was twisted through two right angles, the two routes would become united and re-entrant. If $x$ and $y$ are the numbers of a cell reckoned from two consecutive sides of the board, we may call the cell 
whose distances are respectively $x$ and $y$ from the opposite sides a complementary cell. Thus the cells $(x, y)$ and $(9-x$, $9-y$ ) are complementary, where $x$ and $y$ denote respectively the column and row occupied by the cell. Then in Euler's solution the numbers in complementary cells differ by 32 : for instance, the cell $(3,7)$ is complementary to the cell $(6,2)$, the one is occupied by 57 , the other by 25 .

Roget's method, which is described later, can be also applied to give half-board solutions. The result is indicated above. The close of Euler's memoir is devoted to showing how the method could be applied to crosses and other rectangular figures. I may note in particular his elegant re-entrant symmetrical solution for a square of 100 cells.

The next attempt of any special interest is due to Vandermonde*, who reduced the problem to arithmetic. His idea was to cover the board by two or more independent routes taken at random, and then to connect the routes. He defined the position of a cell by a fraction $x / y$, whose numerator $x$ is the number of the cell from one side of the board, and whose denominator $y$ is its number from the adjacent side of the board; this is equivalent to saying that $x$ and $y$ are the co-ordinates of a cell. In a series of fractions denoting a knight's path, the differences between the numerators of two consecutive fractions can be only one or two, while the corresponding differences between their denominators must be two or one respectively. Also $x$ and $y$ cannot be less than 1 or greater than 8. The notation is convenient, but Vandermonde applied it merely to obtain a particular solution of the problem for a board of 64 cells: the method by which he effected this is analogous to that established by Euler, but it is applicable only to squares of an even order. The route that he arrives at is defined in his notation by the following fractions: $5 / 5,4 / 3,2 / 4,4 / 5,5 / 3,7 / 4,8 / 2,6 / 1,7 / 3$, $8 / 1,6 / 2,8 / 3,7 / 1,5 / 2,6 / 4,8 / 5,7 / 7,5 / 8,6 / 6,5 / 4,4 / 6,2 / 5$, $1 / 7,3 / 8,2 / 6,1 / 8,3 / 7,1 / 6,2 / 8,4 / 7,3 / 5,1 / 4,2 / 2,4 / 1,3 / 3$, $1 / 2,3 / 1,2 / 3,1 / 1,3 / 2,1 / 3,2 / 1,4 / 2,3 / 4,1 / 5,2 / 7,4 / 8,3 / 6$,

- L'Histoirs de l'Acadénie des Sciences for 1771, Paris, 1774, pp. 566-574. 
$4 / 4,5 / 6,7 / 5,8 / 7,6 / 8,7 / 6,8 / 8,6 / 7,8 / 6,7 / 8,5 / 7,6 / 5,8 / 4$, $7 / 2,5 / 1,6 / 3$.

The path is re-entrant but unsymmetrical. Had he transferred the first three fractions to the end of this series he would have obtained two symmetrical circuits of thirty-two moves joined unsymmetrically, and might have been enabled to advance further in the problem. Vandermonde also considered the case of a route in a cube.

In 1773 Collini* proposed the exclusive use of symmetrical routes arranged without reference to the initial cell, but connected in such a manner as to permit of our starting from it. This is the foundation of the modern manner of attacking the problem. The method was re-invented in 1825 by Pratt†, and in 1840 by Roget, and has been subsequently employed by various writers. Neither Collini nor Pratt showed skill in using this method. The rule given by Roget is described later.

One of the most ingenious of the solutions of the knight's path is that given in 1823 by Warnsdorff + . His rule is that the knight must be always moved to one of the cells from which it will command the fewest squares not already traversed. The solution is not symmetrical and not re-entrant; moreover it is difficult to trace practically. The rule has not been proved to be true, but no exception to it is known: apparently it applies also to all rectangular boards which can be covered completely by a knight. It is somewhat curious that in most cases a single false step, except in the last three or four moves, will not affect the result.

Warnsdorff added that when, by the rule, two or more cells are open to the knight, it may be moved to either or any of them indifferently. This is not so, and with great ingenuity two or three cases of failure have been constructed, but it would require exceptionally bad luck to happen accidentally on such a route.

- Solution du Problème du Cavalier au Jeu des Échecs, Mannheim, 1778.

+ Studies of Chess, sixth edition, London, 1825.

‡ H. C. Warnsdorff, Des Rösselsprunges einfuchste und allgemeinste Lösung, Schmalkaldcn, 1823 : *ee also Jaenisch, vol. II, pp. 56-61, 273-289. 
The above methods have been applied to boards of various shapes, especially to boards in the form of rectangles, crosses, and circles*.

All the more recent investigations impose additional restrictions: such as to require that the route shall be re-entrant, or more generally that it shall begin and terminate on given cells.

The simplest solution with which I am acquainted is due to $\mathrm{De}$ Lavernède, but is more generally associated with the name of Roget whose paper in 1840 attracted general notice to it $\dagger$. It divides the whole route into four circuits, which can be combined so as to enable us to begin on any cell and terminate on any other cell of a different colour. Hence, if we like to select this last cell at a knight's move from the initial cell, we obtain a re-entrant route. On the other hand, the rule is applicable only to square boards containing $(4 n)^{2}$ cells : for example, it could not be used on the board of the French jeu des dames, which contains 100 cells.

Roget began by dividing the board of 64 cells into four quarters. Each quarter contains 16 cells, and these 16 cells can be arranged in 4 groups, each group consisting of 4 cells which form a closed knight's path. All the cells in each such path are denoted by the same letter $l, e, a$, or $p$, as the case may be. The path of 4 cells indicated by the consonants $l$ and the path indicated by the consonants $p$ are diamond-shaped: the paths indicated respectively by the vowels $e$ and $a$ are square-shaped, as may be seen by looking at one of the four quarters in figure $\mathrm{i}$ below.

Now all the 16 cells on a complete chess-board which are marked with the same letter can be combined into one circuit, and wherever the circuit begins we can make it end on any other cell in the circuit, provided it is of a different colour to the initial cell. If it is indifferent on what cell the circuit terminates we may make the circuit re-entrant, and

* See ex. gr. T. Ciccolini's work Del Cavallo degli Scacchi, Paris, 1836.

† J. E. T. de Lavernède, Mémoires de l'Académie Royale du Gard, Nimes, 1839, pp. 151-179. P. M. Roget, Philosophical Magazine, April, 1840, series 3, vol. xVI, pp. 305-309; see also the Quarterly Journal of Mathematics for 1877, vol. XIV, pp. 354-359; and the Leisure Hour, Sept. 13, 1873, pp. 587-590, and Dec. 20,1873 , pp. $813-815$.

B. $R$. 
in this case we can make the direction of motion round each group (of 4 cells) the same. For example, all the cells marked $p$ can be arranged in the circuit indicated by the successive numbers 1 to 16 in figure ii below. Similarly all the cells marked $a$ can be combined into the circuit indicated by the numbers 17 to 32 ; all the $l$ cells into the circuit 33 to 48; and all the $e$ cells into the circuit 49 to 64 . Each of the circuits indicated above is symmetrical and re-entrant. The consonant and the vowel circuits are said to be of opposite kinds.

\begin{tabular}{|l|l|l|l|l|l|l|l|}
\hline$l$ & $e$ & $a$ & $p$ & $l$ & $e$ & $a$ & $p$ \\
\hline$a$ & $p$ & $l$ & $e$ & $a$ & $p$ & $l$ & $e$ \\
\hline$e$ & $l$ & $p$ & $a$ & $e$ & $l$ & $p$ & $a$ \\
\hline$p$ & $a$ & $e$ & $l$ & $p$ & $a$ & $e$ & $l$ \\
\hline$l$ & $e$ & $a$ & $p$ & $l$ & $e$ & $a$ & $p$ \\
\hline$a$ & $p$ & $l$ & $e$ & $a$ & $p$ & $l$ & $e$ \\
\hline$e$ & $l$ & $p$ & $a$ & $e$ & $\iota$ & $p$ & $a$ \\
\hline$p$ & $a$ & $e$ & $l$ & $p$ & $a$ & $e$ & $l$ \\
\hline
\end{tabular}

Roget's Solution (i).

\begin{tabular}{|c|c|c|c|c|c|c|c|}
\hline 34 & 51 & 32 & 15 & 38 & 53 & 18 & 3 \\
\hline 31 & 14 & 35 & 52 & 17 & 2 & 39 & 54 \\
\hline 50 & 33 & 16 & 29 & 56 & 37 & 4 & 19 \\
\hline 13 & 30 & 49 & 36 & 1 & 20 & 55 & 40 \\
\hline 48 & 63 & 28 & 9 & 44 & 57 & 22 & 5 \\
\hline 27 & 12 & 45 & 64 & 21 & 8 & 41 & 58 \\
\hline 62 & 47 & 10 & 25 & 60 & 43 & 6 & 23 \\
\hline 11 & 26 & 61 & 46 & 7 & 24 & 59 & 42 \\
\hline
\end{tabular}

Roget's Solution (ii).

The general problem will be solved if we can combine the four circuits into a route which will start from any given cell, and terminate on the 64th move on any other given cell of a different colour. To effect this Roget gave the two following rules.

First. If the initial cell and the final cell are denoted the one by a consonant and the other by a vowel, take alternately circuits indicated by consonants and vowels, beginning with the circuit of 16 cells indicated by the letter of the initial cell and concluding with the circuit indicated by the letter of the final cell.

Second. If the initial cell and the final cell are denoted both by consonants or both by vowels, first select a cell, $Y$, in the same circuit as the final cell, $Z$, and one move from it, next select a cell, $X$, belonging to one of the opposite circuits and one move from $Y$. This is always possible. Then, leaving 
out the cells $Z$ and $Y$, it always will be possible, by the rule already given, to travel from the initial cell to the cell $X$ in 62 moves, and thence to move to the final cell on the 64th move.

In both cases however it must be noticed that the cells in each of the first three circuits will have to be taken in such an order that the circuit does not terminate on a corner, and it may be desirable also that it should not terminate on any of the border cells. This will necessitate some caution. As far as is consistent with these restrictions it is convenient to make these circuits re-entrant, and to take them and every group in them in the same direction of rotation.

As an example, suppose that we are to begin on the cell numbered 1 in figure ii above, which is one of those in a $p$ circuit, and to terminate on the cell numbered 64 , which is one of those in an $e$ circuit. This falls under the first rule: hence first we take the 16 cells marked $p$, next the 16 cells marked $a$, then the 16 cells marked $l$, and lastly the 16 cells marked e. One way of effecting this is shown in the diagram. Since the cell 64 is a knight's move from the initial cell the route is re-entrant. Also each of the four circuits in the diagram is symmetrical, re-entrant, and taken in the same direction, and the only point where there is any apparent breach in the uniformity of the movement is in the passage from the cell numbered 32 to that numbered 33.

A rule for re-entrant routes, similar to that of Roget, has been given by various subsequent writers, especially by De Polignac* and by Laquière $\dagger$, who have stated it at much greater length. Neither of these authors seems to have been aware of Roget's theorems. De Polignac, like Roget, illustrates the rule by assigning letters to the various squares in the way explained above, and asserts that a similar rule is applicable to all even squares.

- Comptes Rendus, April, 1861 ; and Bulletin de la Société Mathématique de France, 1881, vol. Ix, pp. 17-24.

$\uparrow$ Bulletin de la Société Mathématique de France, 1880, vol. viI, pp. 82$102,132-158$. 
Roget's method can be also applied to two half-boards, as indicated in the figure given above on page 126 .

The method which Jaenisch gives as the most fundamental is not very different from that of Roget. It leads to eight forms, similar to that in the diagram printed below, in which the sum of the numbers in every column and every row is 260 ; but although symmetrical it is not in my opinion so easy to reproduce as that given by Roget. Other solutions, notably those by Moon and by Wenzelides, were given in former editions of this work. The two re-entrant routes printed below, each covering 32 cells, and together covering the board, are remarkable as constituting a magic square*.

\begin{tabular}{|c|c|c|c|c|c|c|c|}
\hline 63 & 22 & 15 & 40 & 1 & 42 & 59 & 18 \\
\hline 14 & 39 & 64 & 21 & 60 & 17 & 2 & 43 \\
\hline 37 & 62 & 23 & 15 & 41 & 4 & 19 & 58 \\
\hline 24 & 13 & 38 & 361 & 20 & 57 & 44 & 3 \\
\hline 11 & 36 & 25 & 52 & 29 & 46 & 5 & 56 \\
\hline 26 & 51 & 12 & 33 & 8 & 55 & 30 & 45 \\
\hline 35 & 10 & 49 & 28 & 53 & 32 & 47 & 6 \\
\hline 50 & 27 & 34 & 9 & 48 & 7 & 54 & 31 \\
\hline
\end{tabular}

Jaenisch's Solution.

\begin{tabular}{|c|c|c|c|c|c|c|c|}
\hline 15 & 20 & 17 & 36 & 13 & 64 & 61 & 34 \\
\hline 18 & 37 & 14 & 21 & 60 & 35 & 12 & 63 \\
\hline 25 & 16 & 19 & 44 & 5 & 62 & 33 & 56 \\
\hline 38 & 45 & 26 & 59 & 22 & 55 & 4 & 11 \\
\hline 27 & 24 & 39 & 6 & 43 & 10 & 57 & 54 \\
\hline 40 & 49 & 46 & 23 & 58 & 3 & 32 & 9 \\
\hline 47 & 28 & 51 & 42 & 7 & 30 & 53 & 2 \\
\hline 50 & 41 & 48 & 29 & 52 & 1 & 8 & 31 \\
\hline
\end{tabular}

Two Half Board Solutions.

It is as yet impossible to say how many solutions of the problem exist. Legendre $\nmid$ mentioned the question, but Minding + was the earliest writer to attempt to answer it. More recent investigations have shown that on the one hand the number of possible routes is less $\S$ than the number of combinations of 168 things taken 63 at a time, and on the other hand is greater than 31,054144 --since this latter number is the number of re-entrant paths of a particular type\|.

* Seo A. Rilly, Le Problène du Cavalier des Échecs, Troyes, 1905.

+ Théorie des Nombres, Paris, 2nd edition, 1830, vol. rI, p. 165.

$\ddagger$ Cambridge and Dublin Mathematical Journal, 1852, vol, vII, pp. 147-156; and Crelle's Journal, 1853, vol. xurv, pp. 73-82.

§ Jaenisch, vol. II, p. 268.

॥ Bulletin de la Société Mathématique de France, 1881, vol, IX, pp. 1-17. 
Analogous Problems. Similar problems can be constructed in which it is required to determine routes by which a piece moving according to certain laws (ex. gr. a chess-piece such as a king, \&c.) can travel from a given cell over a board so as to occupy successively all the cells, or certain specified cells, once and only once, and terminate its route in a given cell. Euler's method can be applied to find routes of this kind: for instance, he applied it to find a re-entrant route by which a piece that moved two cells forward like a castle and then one cell like a bishop would occupy in succession all the black cells on the board.

King's Re-Entrant Path. As one example here is a reentrant tour of a king which moves successively to every cell

\begin{tabular}{|r|r|r|r|r|r|r|r|}
\hline 61 & 62 & 63 & 64 & 1 & 2 & 3 & 4 \\
\hline 60 & 11 & 58 & 57 & 8 & 7 & 54 & 5 \\
\hline 12 & 59 & 10 & 9 & 56 & 55 & 6 & 53 \\
\hline 13 & 14 & 15 & 16 & 49 & 50 & 51 & 53 \\
\hline 20 & 19 & 18 & 17 & 48 & 47 & 40 & 45 \\
\hline 21 & 38 & 23 & 24 & 41 & 42 & 27 & 44 \\
\hline 37 & 22 & 39 & 40 & 25 & 26 & 43 & 28 \\
\hline 36 & 35 & 34 & 33 & 32 & 31 & 30 & 39 \\
\hline
\end{tabular}

King's Magic Tour on a Chess-Board.

of the board. I give it because the numbers indicating the cells successively occupied form a magic square. Of course this also gives a solution of a re-entrant route of a queen covering the board.

Rook's Re-Entrant Path. There is no difficulty in constructing re-entrant tours for a rook which moves successively to every cell of the board. For instance, if the rook starts from the cell 11 it can move successively to the cells $18,88,81,71$, $77,67,61,51,57,47,41,31,37,27,21$; and so back to 11 : this is a symmetrical route. Of course this also gives a solution of a re-entrant route for a king or a queen covering the board. 
If we start from any of the cells mentioned above, the rook takes sixteen moves. If we start from any cell in the middle of one of these moves, it will take seventeen moves to cover this route, but I believe that in most cases wherever the initial cell be chosen sixteen moves will suffice, though in general the route will not be symmetrical. On a board of $n^{2}$ cells it is possible to find a route by which a rook can move successively from its initial cell to every other cell once and only once. Moreover* starting on any cell its path can be made to terminate, if $n$ be even, on any other cell of a different colour, and, if $n$ be odd, on any other cell of the same colour.

Bishop's Re-Entrant Path. As yet another instance, a bishop can traverse all the cells of one colour on the board in seventeen moves if the initial cell is properly chosent; for instance, starting from the cell 11, it may move successively to the cells $55,82,71,17,28,46,13,31,86,68,57,48,15,51,84,66,88$. One more move will bring it back to the initial cell. From the nature of the case, it must traverse some cells more than once.

Miscellaneous Problems. We may construct numerous such problems concerning the determination of routes which cover the whole or part of the board subject to certain conditions. I append a few others which may tax the ingenuity of those not accustomed to such problems.

Routes on a Chess-Board. One of the simplest is the determination of the path taken by a rook, placed in the cell 11, which moves, one cell at a time, to the cell 88 , so that in the course of its path it enters every cell once and only once. This can be done, though I have seen good mathematicians puzzled to effect it. A hasty reader is apt to misunderstand the conditions of the problem.

Another simple problem of this kind is to move a queen from the cell 33 to the cell 66 in fifteen moves entering every cell once

* L'Intermédiaive des Mathématiciens, Paris, 1901, vol. vII, pp. 153-154.

† H. E. Dudeney, The Tribune, Deo. 3, 1906. 
and only once, and never crossing its own track or entering a cell more than once*.

A somewhat similar, but more difficult, question is the determination of the greatest distance which can be travelled by a queen starting from its own square in five consecutive moves, subject to the condition that it never crosses its own track or enters a cell more than oncef. In calculating the distance it may be assumed that the paths go through the centres of the cells. If the length of the side of a cell is one inch, the distance exceeds 33.97 inches.

Another familiar problem can be enunciated as follows. Construct a rectangular board of $m n$ cells by ruling $m+1$ vertical lines and $n+1$ horizontal lines. It is required to know how many routes can be taken from the top left-hand corner to the bottom right-hand corner, the motion being along the ruled lines and its direction being always either vertically downwards or horizontally from left to right. The answer is the number of permutations of $m+n$ things, of which $m$ are alike of one kind and $n$ are alike of another kind: this is equal to $(m+n) ! / m ! n !$. Thus on a square board containing 16 cells (i.e. one-quarter of a chess-board), where $m=n=4$, there are 70 such routes; while on a common chess-board, where $m=n=8$, there are no less than 12870 such routes. A rook, moving according to the same law, can travel from the top left-hand cell to the bottom right-hand cell in $(m+n-2) ! /(m-1) !(n-1)$ ! ways. Similar theorems can be enunciated for a parallelopiped.

Another question of this kind is the determination of the number of closed routes through $m n$ points arranged in $m$ rows and $n$ columns, following the lines of the quadrilateral net-work, and passing once and only once through each point+.

Guarini's Problem. One of the oldest European problems connected with the chess-board is the following which was

* H. E. Dudeney, The Tribune, Oct. 3, 1906.

† Ibid., Oct. 2, 1906.

¥ See C. F. Sainte-Marie in L'Intermédiaire des Mathêmaticiens, Paris, vol. xI, March, 1904, pp. 86-88. 
propounded in 1512. It was quoted by Lucas in 1894, but I believe has not been published otherwise than in his works and the earlier editions of this book. On a board of nine cells, such as that drawn below, the two white knights are placed on the

\begin{tabular}{|c|c|c|}
\hline$a$ & $C$ & $d$ \\
\hline$D$ & & $B$ \\
\hline$b$ & $A$ & $c$ \\
\hline
\end{tabular}

two top corner cells $(a, d)$, and the two black knights on the two bottom corner cells $(b, c)$ : the other cells are left vacant. It is required to move the knights so that the white knights shall occupy the cells $b$ and $c$, while the black shall occupy the cells $a$ and $d$. The solution is obvious.

Queens' Problem. Another problem consists in placing sixteen queens on a board so that no three are in a straight line *. One solution is to place them on the cells $15,16,25,26$, $31,32,41,42,57,58,67,68,73,74,83,84$. It is of course assumed that each queen is placed on the middle of its cell.

Latin Squares. Another problem of the chess-board type is the determination of the number $x_{n}$ of Latin Squares of any assigned order $n$ : a Latin Square of the $n$th order being defined as a square of $n^{2}$ cells (in $n$ rows and $n$ columns) in which $n^{2}$ letters consisting of $n$ " $a$ 's," $n$ " $b$ 's,"..., are arranged in the cells so that the $n$ letters in each row and each column are different. The general theory is difficult $\uparrow$, but it may amuse my readers to verify the following results for some of the lower values of $n: x_{2}=2, x_{8}=6, x_{4}=576, x_{5}=149760$. Clearly $x_{n}$ is a multiple of $n !(n-1)$ !

* H. E. Dudeney, The Tribune, November 7, 1906.

+ See P. A. MacMahon, Combinatory Analysis, Cambridge, 1915-16, vol. I, pp. 246-263; vol. II, pp. 323-326. 


\section{CHAPTER VII.}

\section{MAGIC SQUARES.}

A Magic Square consists of a number of integers arranged in the form of a square, so that the sum of the numbers in every row, in every column, and in each diagonal is the same. If the integers are the consecutive numbers from 1 to $n^{2}$ the square is said to be of the $n$th order, and it is easily seen that in this case the sum of the numbers in every row, column, and diagonal is equal to $\frac{1}{2} n\left(n^{2}+1\right)$ : this number may be denoted by $N$. Unless otherwise stated, I confine my account to such magic squares, that is, to squares formed with consecutive integers from 1 upwards. The same rules cover similar problems with $n^{2}$ numbers in arithmetical progression.

Thus the first 16 integers, arranged in either of the forms in figures $i$ and ii below, represent magic squares of the fourth

\begin{tabular}{|c|c|c|c|}
\hline 16 & 3 & 2 & 13 \\
\hline 5 & 10 & $\frac{11}{8}$ & $\frac{8}{12}$ \\
\hline 9 & 6 & 7 & $\frac{12}{1}$ \\
\hline 4 & 15 & 14 & 1 \\
\hline
\end{tabular}

Figure i. $n=4$.

\begin{tabular}{|c|c|c|c|}
\hline 15 & 10 & 3 & 6 \\
\hline 4 & 5 & 16 & 9 \\
\hline 14 & 11 & 2 & 7 \\
\hline 1 & 8 & 13 & 12 \\
\hline
\end{tabular}

Figure ii. $n=4$.

order, the sum of the numbers in each row, column, and diagonal being 34. Similarly figure iii on page 139, figure xiii on page 145, and figure xvi on page 151 represent magic squares of the fifth order; figure vi on page 141 represents a magic square of the sixth order; figure xviii on page 154 represents a magic square of the seventh order; figure xii on page 143 and figure xxii on page 157 represent magic squares of the eighth order; figure xxiii on page 157 represents a magic square of the ninth 
order; and figure ix on page 142 represents a magic square of the tenth order.

The formation of these squares is an old amusement, and in times when mystical ideas were associated with particular numbers it was natural that such arrangements should be studied. Magic squares were constructed in India before the Christian era: their introduction into Europe appears to have been due to Moschopulus, who lived at Constantinople in the early part of the fifteenth century. The famous Cornelius Agrippa (1486-1535) constructed magic squares of the orders $3,4,5,6,7,8,9$, which were associated by him with the seven astrological "planets": namely, Saturn, Jupiter, Mars, the Sun, Venus, Mercury, and the Moon. A magic square engraved on a silver plate was sometimes prescribed as a charm against the plague, and one, namely, that represented in figure $i$ on the last page, is drawn in the picture of Melancholy, painted in 1514 by Albert Duirer: the numbers in the middle cells of the bottom row giving the date of the work. The mathematical theory of the construction of these squares was taken up in France in the seventeenth century, and later has been a favourite subject with writers in many countries*.

It is convenient to use the following terms. The spaces or small squares occupied by the numbers are called cells. It is customary to call the rows first, second, etc., reckoning from the top, and the columns first, second, etc., reckoning from the left. The $h$ th and $(n+1-h)$ th rows (or columns) are said to be complementary. The $k$ th cell in the $h$ th row is said to be sleewly related to the $(n+1-k)$ th cell in the $(n+1-h)$ th row. Skewly related cells are situated symmetrically to the centre of the square.

Magic Squares of any order higher than two can be constructed at sight. The rule to be used varies according as the order $n$ is odd, that is, of the form $2 m+1$; or singly-even, that is, of the form $2(2 m+1)$; or doubly-even, that is, of the form

* For a sketch of the history of the subject and its bibliography see S. Günther's Geschichte der mathematischen Wissenschaften, Leipzig, 1876, chapter iv; and W. Ahrens, Mathematische Unterhaltungen und Spiele, Leipzig, 1901, chapter rii. 
$4 m$. In each case, I now give the simplest rule with which I am acquainted, omitting alternative methods described in previous editions of this work.

Magic Squares of an Odd Order. A magic square of the $n$th order, where $n=2 m+1$, can be constructed by the following rule due to De la Loubère*. First, the number 1 is placed in the middle cell of the top row. The successive numbers are then placed in their natural order in a diagonal line which slopes upwards to the right, except that (i) when the top row is reached the next number is written in the bottom row as if it came immediately above the top row; (ii) when the righthand column is reached, the next number is written in the left-hand column, as if it immediately succeeded the right-hand column; and (iii) when a cell which has been filled up already, or when the top right-hand square is reached, the path of the series drops to the row vertically below it and then continues to mount again. Probably a glance at the diagram in figure iii, showing the construction by this rule of a square of the fifth order, will make the rule clear.

\begin{tabular}{|c|c|c|c|c|}
\hline 17 & 24 & 1 & 8 & 15 \\
\hline 23 & 5 & 7 & 14 & 16 \\
\hline 4 & 6 & 13 & 20 & 22 \\
\hline 10 & 12 & 19 & 21 & 3 \\
\hline 11 & 18 & 25 & 2 & 9 \\
\hline
\end{tabular}

Figure iii. $n=5$.

\begin{tabular}{|c|c|c|c|c|}
\hline $15+2$ & $20+4$ & $0+1$ & $5+3$ & $10+5$ \\
\hline $20+3$ & $0+5$ & $5+2$ & $10+4$ & $15+1$ \\
\hline $0+4$ & $5+1$ & $10+3$ & $15+5$ & $20+2$ \\
\hline $5+5$ & $10+2$ & $15+4$ & $20+1$ & $0+3$ \\
\hline $10+1$ & $15+3$ & $20+5$ & $0+2$ & $5+4$ \\
\hline
\end{tabular}

Figure iv. $n=5$.

The reason why such a square is magic can be best explained by taking a particular case, for instance, $n=5$, and expressing all the numbers in the scale of notation whose radix is 5 (or $n$, if the magic square is of the order $n$ ), except that 5 is allowed to appear as a unit-digit and 0 is not allowed to appear as a unit-digit. The result is shown in figure iv. From that figure it will be seen that the method of construction ensures that

* S. De la Loubère, Du Royaume de Siam (Eng. Trans.), London, 1693, vol. Ir, pp. 227-247. De la Loubere was the envoy of Louis XIV to Siam in 1687-8, and there learnt this method. 
every row and every column shall contain one and only one of each of the unit-digits $1,2,3,4,5$, the sum of which is 15 ; and also one and only one of each of the radix-digits $0,5,10,15,20$, the sum of which is 50 . Hence, as far as rows and columns are concerned, the square is magic. Moreover if the square is odd, each of the diagonals will contain one and only one of each of the unit-digits $1,2,3,4,5$. Also the leading diagonal will contain one and only one of the radix-digits $0,5,10,15,20$, the sum of which is 50 ; and if, as is the case in the square drawn above, the number 10 is the radix-digit to be added to the unit-digits in the other diagonal, then the sum of the radixdigits in that diagonal is also 50. Hence the two diagonals also possess the magical property.

The effect of the construction is that we superpose two magic squares, one made with the unit-digits $1,2,3,4$, 5, each repeated five times, and the other with the radix-digits $0,5,10$, 15,20 , each repeated five times. These squares being magic, the square resulting from their superposition must be magic, and they are so formed that their superposition ensures that every number from 1 to 25 appears once and only once in the resulting square.

Magic Squares of a Singly-Even Order. A magic square of the $n$th order, where $n=2(2 m+1)$, can be constructed by the following rule due to Ralph Strachey*. Divide the square into four equal quarters $A, B, C, D$, as shown in figure $\mathrm{x}$ on page 143. Construct in $A$, by De la Loubère's method, a magic square with the numbers 1 to $u^{2}$ where $u=n / 2$. Construct by the same rule, in $B, C, D$, similar magic squares with the numbers $u^{2}+1$ to $2 u^{2}, 2 u^{2}+1$ to $3 u^{2}$, and $3 u^{2}+1$ to $4 u^{2}$. Clearly the resulting composite square is magic in columns. In the middle row of $A$ take the $m$ cells next but one to the left-hand side, and in each of the other rows take the $m$ cells nearest to the left-hand side, interchange the numbers in these cells with the numbers in the corresponding cells in $D$. Next interchange the numbers in the cells in each of the $m+2$ columns next to the left-hand side of $B$ with the numbers in the corresponding

- Communicated to me in a letter, August 1918. 
cells in $C$. Of course the resulting square remains magic in columns. It will also now be magic in rows and diagonals, since the construction is equivalent to writing in each of the quarters $A, B, C, D$, equal magic squares of the order $u$ made with the numbers 1 to $u^{2}$, and then superposing on them a magic square of the $n$th order made with the four radix numbers $0, u^{2}$, $2 u^{2}, 3 u^{2}$, each repeated $u^{2}$ times. The component squares being magic, the square resulting from their superposition must be magic, and they are so formed that their superposition ensures that every number from 1 to $n^{2}$ appears once and only once in the resulting square.

Figures $\nabla$ and vi show the application of the rule to the construction of a magic square of the sixth order. In figure $v$,

\begin{tabular}{|c|c|c|c|c|c|}
\hline 8 & 1 & 6 & $\underline{17}$ & $\underline{10}$ & $\frac{15}{16}$ \\
\hline 3 & $\frac{5}{7}$ & 7 & $\underline{\underline{12}}$ & $\frac{14}{-14}$ & $\frac{\underline{16}}{-}$ \\
\hline$\underline{4}$ & 9 & 2 & $\underline{13}$ & $\underline{-18}$ & $\underline{11}$ \\
\hline 35 & 28 & 33 & 26 & 19 & 24 \\
\hline 30 & 32 & 34 & 21 & 23 & 25 \\
\hline 31 & 36 & 29 & 22 & 27 & 20 \\
\hline
\end{tabular}

Figure v. Initial Quarter-Squares.

\begin{tabular}{|c|c|c|c|c|c|}
\hline 35 & 1 & 6 & 26 & 19 & 24 \\
\hline 3 & 32 & 7 & 21 & 23 & 25 \\
\hline 31 & 9 & 2 & 22 & 27 & 20 \\
\hline 8 & 28 & 33 & 17 & 10 & 15 \\
\hline 30 & 5 & $\frac{34}{12}$ & 14 & 16 \\
\hline 4 & 36 & 29 & 13 & 18 & 11 \\
\hline
\end{tabular}

Figure vi. Final Square, $n=6$.

\begin{tabular}{|c|c|c|c|c|c|}
\hline $27+8$ & $0+1$ & $0+6$ & $18+8$ & $18+1$ & $18+6$ \\
\hline $0+3$ & $27+5$ & $0+7$ & $18+3$ & $18+5$ & $18+7$ \\
\hline $27+4$ & $0+9$ & $0+2$ & $18+4$ & $18+9$ & $18+2$ \\
\hline $0+8$ & $27+1$ & $27+6$ & $9+8$ & $9+1$ & $9+6$ \\
\hline $27+3$ & $0+5$ & $27+7$ & $9+3$ & $9+5$ & $9+7$ \\
\hline $0+4$ & $27+9$ & $27+2$ & $9+4$ & $9+9$ & $9+2$ \\
\hline
\end{tabular}

Figure vii. Final Square, $n=6$.

those numbers in the cells in the initial quarter-squares $A$ and $B$ which are to be interchanged vertically with the numbers in the corresponding cells in $D$ and $C$ are underlined; figure vi represents the final square obtained; and figure vii shows how 
these interchanges serve to bring the radix-digits to a position which makes the square magic.

Since this construction is novel, I add figures to show the application of the rule to the formation of a square of the tenth order: in figure viii those numbers in the central quarter-

\begin{tabular}{|c|c|c|c|c|c|c|c|c|c|}
\hline 17 & 24 & 1 & 8 & 15 & 42 & $\underline{49}$ & 26 & 33 & 40 \\
\hline 23 & 5 & 7 & 14 & 16 & 48 & 30 & 32 & 39 & 41 \\
\hline 4 & 6 & 13 & 20 & 22 & 29 & 31 & 38 & 45 & 47 \\
\hline 10 & 12 & 19 & 21 & 3 & 35 & 37 & 44 & 46 & 28 \\
\hline 11 & 18 & 25 & 2 & 9 & 36 & $\underline{43}$ & 50 & 27 & 34 \\
\hline 92 & 99 & 76 & 83 & 90 & 67 & 74 & 51 & 58 & 65 \\
\hline 98 & 80 & 82 & 89 & 91 & 73 & 55 & 57 & 64 & 66 \\
\hline 79 & 81 & 88 & 95 & 97 & 54 & 56 & 63 & 70 & 72 \\
\hline 85 & 87 & 94 & 96 & 78 & 60 & 62 & 69 & 71 & 53 \\
\hline 86 & 93 & 100 & 77 & 84 & 61 & 68 & 75 & 52 & 59 \\
\hline
\end{tabular}

Figure viii. Initial Quarter-Squares, $n=10$.

\begin{tabular}{|c|c|c|c|c|c|c|c|c|c|}
\hline 92 & 99 & 1 & 8 & 15 & 67 & 74 & 51 & 58 & 40 \\
\hline 98 & 80 & 7 & 14 & 16 & 73 & $\frac{55}{57}$ & 64 & 41 \\
\hline 4 & 81 & $\frac{88}{20}$ & $\frac{22}{54}$ & 56 & 63 & 70 & 47 \\
\hline 85 & $\frac{87}{19}$ & $\frac{21}{3}$ & $\frac{3}{60}$ & $\frac{62}{69}$ & $\frac{71}{28}$ \\
\hline 86 & $\frac{93}{95}$ & $\frac{25}{2}$ & 9 & 61 & 68 & 75 & 52 & 34 \\
\hline 17 & 24 & 76 & 83 & 90 & 42 & 49 & 26 & 33 & 65 \\
\hline 23 & 5 & 82 & 89 & 91 & 48 & 30 & 32 & 39 & 66 \\
\hline 79 & 6 & 13 & 95 & 97 & 29 & 31 & 38 & 45 & 72 \\
\hline 10 & 12 & 94 & 96 & 78 & 35 & 37 & 44 & 46 & 53 \\
\hline 11 & 18 & 100 & 77 & 84 & 36 & 43 & 50 & 27 & 59 \\
\hline
\end{tabular}

Figure ix. Final Square, $n=10$.

squares $A$ and $B$ which have to be interchanged vertically with the numbers in the corresponding cells in $D$ and $C$ are under- 
lined, while figure ix represents the final nlagic square of the tenth order thus obtained.

Magic Squares of a Doubly-Even Order. A magic square of the $n$th order, where $n=4 m$, can be constructed by the following rule*. Begin by filling the cells of the square with the numbers $1,2, \ldots, n^{2}$, written in their natural order from left to right and taking the rows in succession from the top. Divide the square into four equal quarters $A, B, C, D$, as represented in figure $x$. Divide each of these quarter-squares again into four equal parts, each of which will contain $m^{2}$ cells, as shown in figure xi. Then if the numbers in the cells $a_{1}$ and $a_{4}$ are interchanged with those in the skewly related cells in $C$,

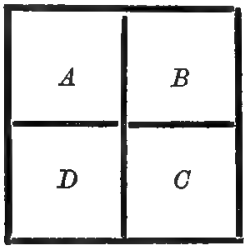

Figure $\mathbf{x}$.

\begin{tabular}{|l|l|l|l|}
\hline$a_{1}$ & $a_{2}$ & $b_{1}$ & $b_{2}$ \\
\hline$a_{3}$ & $a_{4}$ & $b_{3}$ & $b_{4}$ \\
\hline$d_{1}$ & $d_{2}$ & $c_{1}$ & $c_{2}$ \\
\hline$d_{3}$ & $d_{4}$ & $c_{3}$ & $c_{4}$ \\
\hline
\end{tabular}

Figure xi.

\begin{tabular}{|c|c|c|c|c|c|c|c|}
\hline 1 & 2 & 62 & 61 & 60 & 59 & 7 & 8 \\
\hline 9 & 10 & 54 & 53 & 52 & 51 & 15 & 16 \\
\hline 48 & 47 & 19 & 20 & 21 & 22 & 42 & 41 \\
\hline 40 & 39 & 27 & 28 & 29 & 30 & 34 & 33 \\
\hline 32 & 31 & 35 & 36 & 37 & 38 & 26 & 25 \\
\hline 24 & 23 & 43 & 44 & 45 & 46 & 18 & 17 \\
\hline 49 & 50 & 14 & 13 & 12 & 11 & 55 & 56 \\
\hline 57 & 58 & 6 & 5 & 4 & 3 & 63 & 64 \\
\hline
\end{tabular}

Figure xii. A Magic Square, $n=8$.

and the numbers in the cells $b_{2}$ and $b_{3}$ are interchanged with those in the skewly related cells in $D$, the resulting square will be magic. A magic square of the eighth order constructed by this rule is shown in figure xii.

* The rule seems to have been first enunciated in 1889 by W. Firth, but later was independently discovered by various writers: see the Messenger of Mathematics, Cambridge, September, 1893, vol. xxirI, pp. 65-69, and the Monist, Chicago, 1912, vol. xxIr, pp. 53-81. 
The reason for the rule is as follows. In the original formation the sum of the numbers in the $x$ th row is $N-n^{2}(n-2 x+1) / 2$ and the sum of those in the complementary row is $N+n^{2}(n-2 x+1) / 2$. Also the number in any cell in the $x$ th row is less than the number in the corresponding cell in the complementary row by $n(n-2 x+1)$. Hence, if in these two rows we make $n / 2$ interchanges of the numbers in corresponding cells, we increase the sum of the numbers in the $x$ th row by $n \times n(n-2 x+1) / 2$ and therefore make that row magic; while we decrease the sum of the numbers in the complementary row by the same number, and therefore make that row magic. Hence, if in every pair of complementary rows we make $n / 2$ such interchanges, the resulting square will be magic in rows. Similarly for the columns, the sum of the numbers in the $y$ th column is $N-n(n-2 y+1) / 2$, and the sum of the numbers in the complementary column is $N+(n-2 y+1) / 2$; also the number in any cell in the $y$ th column is less than that in the corresponding cell in the complementary column by $n-2 y+1$. Hence, if in these two columns we make $n / 2$ interchanges of numbers in corresponding cells, we make these columns magic; and if we do this for every pair of complementary columns, the resulting square will be magic in columns. But in order that the diagonals may, after these interchanges, remain magic, we must leave the numbers in their cells unaltered or reversed.

The $2 m$ interchanges we made of numbers in the skewly related cells in $a_{1}$ and $c_{4}, a_{4}$ and $c_{1}, b_{2}$ and $d_{3}, b_{3}$ and $d_{2}$ are equivalent to making $2 m$ interchanges in every column, and reversing the diagonals. Hence it will make the square magic. We might equally well have interchanged the cells in $a_{2}, a_{3}, b_{1}, b_{4}$, with those skewly related to them in $C$ and $D$.

Bordered SquARES. One other general method, due to Frénicle, of constructing magic squares of any order should be mentioned. By this method, to form a magic square of the $n$th order we first construct one of the $(n-2)$ th order, add to every number in it an integer, and then surround it with a border of the remaining numbers in such a way as to make the resulting square magic. In this manner from the magic square of the 
3rd order we can build up successively squares of the orders 5,7 , 9, etc., that is, every odd magic square. Similarly from a magic square of the 4th order we can build up successively all higher even magic squares.

The method of construction will be clear if $I$ explain how the square in figure xiii, where $n=5$, is built up. First the inner magic square of the $(n-2)$ th order is formed by any rule we like to choose: the sum of the numbers in each line being $(n-2)\left\{(n-2)^{2}+1\right\} / 2$. To every number in it, $2 n-2$ is added: thus the sum of the numbers in each row, column, and diagonal is now $(n-2)\left\{n^{2}+1\right\} / 2$. The numbers not used are $1,2, \ldots$,

\begin{tabular}{|c|c|c|c|c|}
\hline 2 & 25 & 23 & 7 & 8 \\
\hline 4 & 16 & 9 & 14 & 22 \\
\hline 21 & 11 & 13 & 15 & 5 \\
\hline 20 & 12 & 17 & 10 & 6 \\
\hline 18 & 1 & 3 & 19 & 24 \\
\hline
\end{tabular}

Figure xiii. A Bordered Square, $n=5$.

$2 n-2$, and their complements, $n^{2}, n^{2}-1, \ldots, n^{2}-2 n+3$. These reserved numbers are placed in the $4(n-1)$ border cells so that complementary numbers occur at the end of each row, column, and diagonal of the inner square: this makes the sum of the numbers in each of these latter lines equal to $n\left(n^{2}+1\right) / 2$. It only remains to make the sum of the numbers in each of the border lines also have this value: such an arrangement is easily made by trial and error. With a little patience a magic square of any order can be thus built up, border upon border, and of course it will have the property that, if each border is successively stripped off, the remaining square will still be magic. This is a method of construction much favoured by self-taught mathematicians.

Definite rules for arranging the numbers in the border cells have been indicated*, though not, as far as I know, in a pre-

* For instance, see Japanese Mathematics by D. E. Smith and Y. Mikami, Chicago, 1914, pp. 116-120: in this work, in the diagram on p. 120, the numbers 8 and 29 should be interchanged.

D. $\mathbb{R}$. 
cise form. One rule in the case of an odd square, where $n=2 m+1$, may be put as follows. First, write in the $n$ cells in the top row from left to right the numbers $2,1, m+1, \ldots$, $2 m-1,3 m+1,3 m+2, \ldots, 4 m$. Next, write in the $n$ cells in the left-hand column from top to bottom the numbers $2,3, \ldots, m$, $2 m, 2 m+1, \ldots, 3 m, 4 m^{2}+2$. Fill up the remaining border cells with the proper complementary numbers. Next take the $m$ numbers in the upper row immediately to the right of the lefthand top corner cell, and the $m$ numbers in the left-hand column immediately above the left-hand bottom corner cell. Interchange the numbers in these $2 m$ cells with the numbers in their complementary cells. The resulting square is magic. It may interest my readers to see if they can evolve a similar simple rule for the formation of bordered even squares.

Alternative methods of constructing simple magic squares are constantly being expounded, so the subject is not exhausted, but there is no occasion to go here into further details.

Number of Squares of a Given Order. One unsolved problem in the theory is the determination of the number of magic squares of the fifth (or any higher) order. There is, in effect, only one magic square of the third order, though by reflexions and rotations it can be presented in 8 forms. There are 880 magic squares of the fourth order, but by reflexions and rotations these can be presented in 7040 forms. The problem of the number of magic squares of an order higher than five is unsolved. From the square given above in figure iii on page 139 formed by De la Loubère's method, we can get 720 other distinct squares, for we can permute the symbols 1, 2, 3, 4,5 , in 5 ! ways, and combine with any of these squares any of the 4 ! squares obtained by permuting the symbols $0,5,15,20$. We thus obtain 2880 magic squares of the fifth order, though only 720 of them are distinct. Bachet gave a somewhat similar construction in which he began by placing 1 in the cell immediately above the middle cell, and wrote the consecutive numbers in a line sloping downwards : his method gives another 720 distinct magic squares of the fifth order. There are however numerous other rules for constructing odd magic squares, and 
De la Hire showed that by methods known in his day and apart from mere reflexions and rotations, there were 57600 magic squares of the fifth order which could be formed by the methods he enumerated, and taking account of other methods, it is now known that the total number of magic squares of the fifth order considerably exceeds three-quarters of a million.

Product Squares. Before leaving this part of the subject, I may mention that Montucla suggested the construction of squares whose cells are occupied by numbers such that the product of the numbers in each row, column, and diagonal is constant. The formation of such figures is immediately deducible from that of magic squares, for if the consecutive numbers, namely $1,2,3$, \&c., in a magic square are replaced by consecutive powers of any number $m$, namely $m, m^{2}, m^{3}$, \&c., the products of the numbers in every line will be magic. This is obvious, for if the numbers in any line of a square are $a, a^{\prime}$, $a^{\prime \prime}, \& c$., such that $\Sigma a$ is constant for every line in the square, then $\Pi m^{a}$ is also constant.

Magic Stars. Some elegant magic constructions on starshaped figures (pentagons, hexagons, \&c.) may be noticed in

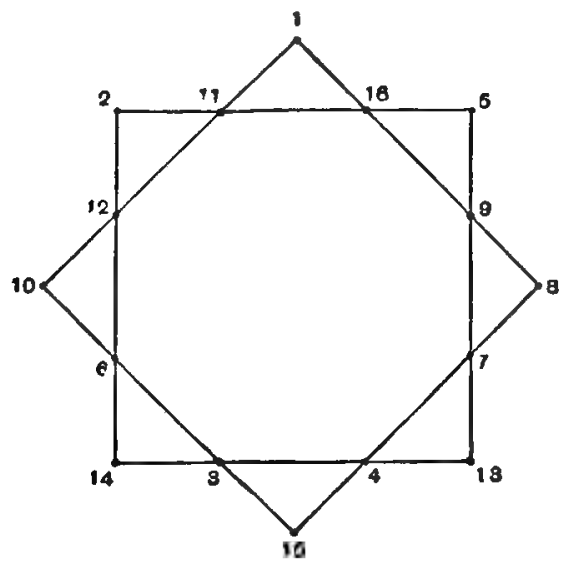

Figure ziv. A Magic Star.

passing, though I will not go into details. One instance will suffice. Suppose a re-entrant octagon is constructed by the 
intersecting sides of two equal concentric squares. It is required to place the first 16 natural numbers on the corners and points of intersection of the sides so that the sum of the numbers on the corner of each square and the sum of the numbers on every side of each square is equal to 34. Eighteen fundamental solutions exist. One of these is given above*.

There are magic circles, rectangles, crosses, diamonds, and other figures: also magic cubes, cylinders, and spheres. The theory of the construction of such figures is of no value, and I cannot spare the space to describe rules for forming them.

Magic Pencils. Hitherto I have concerned myself with numbers arranged in lines. By reciprocating the figures composed of the points on which the numbers are placed we obtain a collection of lines forming pencils, and, if these lines be numbered to correspond with the points, the pencils will be magic + . Thus, in a magic square of the $n$th order, we arrange $n^{2}$ consecutive numbers to form $2 n+2$ lines, each containing $n$ numbers so that the sum of the numbers in each line is the same. Reciprocally we can arrange $n^{2}$ lines, numbered consecutively to form $2 n+2$ pencils, each containing $n$ lines, so that in each pencil the sum of the numbers designating the lines is the same.

For instance, figure $x i i$ on page 143 represents a magic square of 64 consecutive numbers arranged to form 18 lines, each of 8 numbers. Reciprocally, figure $\mathrm{xv}$ represents 64 lines arranged to form 18 pencils, each of 8 lines. The method of construction is fairly obvious. The eight-rayed pencil, vertex 0 , is cut by two parallels perpendicular to the axis of the pencil, and all the points of intersection are joined cross-wise. This gives 8 pencils, with vertices $A, B, \ldots, H ; 8$ pencils, with vertices $A^{\prime}, \ldots, H^{\prime}$; one pencil with its vertex at $O$; and one pencil with its vertex on the axis of the last-named pencil.

The sum of the numbers in each of the $\mathbf{1 8}$ lines in figure xii is the same. To make figure $\mathrm{xV}$ correspond to this we must number the lines in the pencil $A$ from left to right, $1,9, \ldots, 57$,

* Communicated to me by R. Strachey.

† See Magic Reciprocals by G. Frankenstein, Cincinnati, 1875. 
following the order of the numbers in the first column of the square: the lines in pencil $B$ must be numbered similarly to correspond to the numbers in the second column of the square, and so on. To prevent confusion in the figure I have not inserted the numbers, but it will be seen that the method of construction ensures that the sum of the 8 numbers which designate the lines in each of these 18 pencils is the same.

We can proceed a step further, if the resulting figure is cut by two other parallel lines perpendicular to the axis, and if all

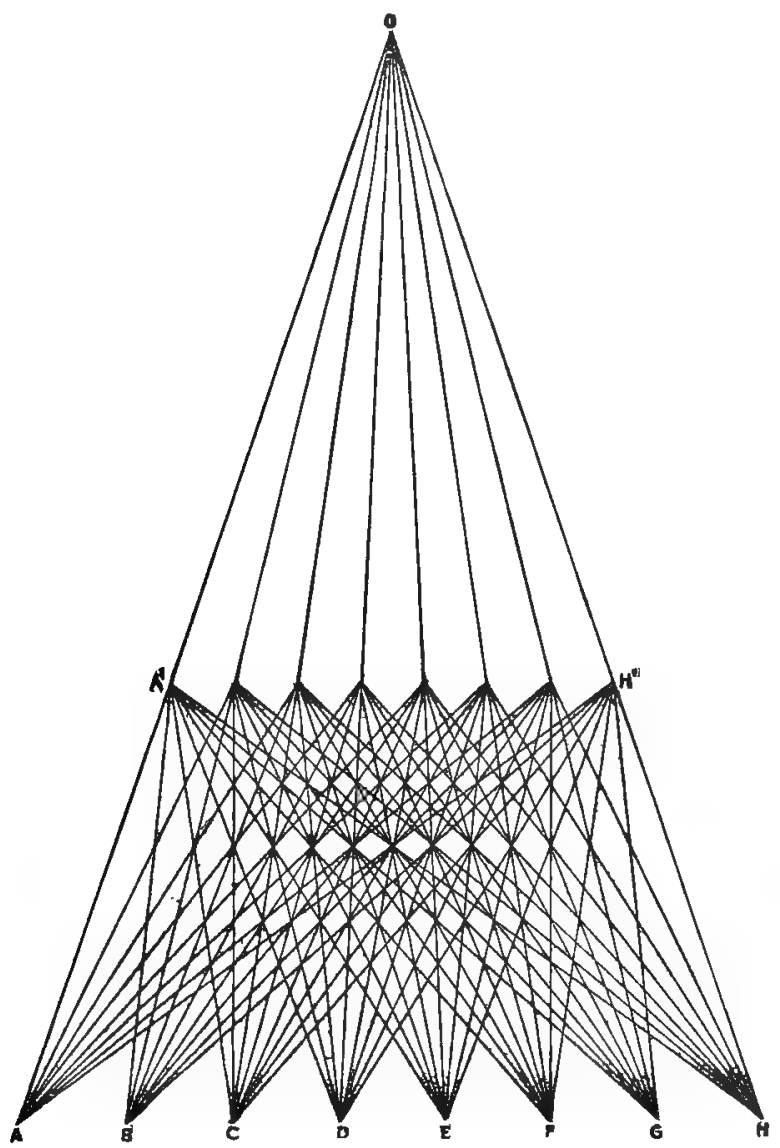

Figure $\mathbf{x \nabla}$ A Magic Pencil, $n=8$. 
the points of their intersection with the cross-joins be joined cross-wise, these new cross-joins will intersect on the axis of the original pencil or on lines perpendicular to it. The whole figure will now give $8^{3}$ lines, arranged in 244 pencils each of 8 rays, and will be the reciprocal of a magic cube of the 8th order. If we reciprocate back again we obtain a representation in a plane of a magic cube.

HYPER-MAGIC SQUARES. With the exception of determining the number of squares of a given order, we may fairly say that the theory of the construction of magic squares, as defined above, has been sufficiently worked out. Accordingly attention has of late been chiefly directed to the construction of squares which, in addition to being magic, satisfy other conditions. I term such squares hyper-magic. Of hyper-magic squares, I will deal only with the theory of Pan-Diagonal and of Symmetrical Squares, though I will describe without going into details what are meant by Doubly and Trebly Magic Squares.

Pandiagonal Squares. One of the earliest additional conditions to be suggested was that the square should be magic along the broken diagonals as well as along the two ordinary diagonals*. Such squares are called Pandiagonal. They are also known as nasik, perfect, and diabolic squares.

For instance, a magic pandiagonal square of the fourth order is represented in figure ii on page 137. In it the sum of the numbers in each row, column, and in the two diagonals is 34 , as also is the sum of the numbers in the six broken diagonals formed by the numbers $15,9,2,8$, the numbers $10,4,7,13$, the numbers $3,5,14,12$, the numbers $6,4,11,13$, the numbers $3,9,14,8$, and the numbers $10,16,7,1$.

It follows from the definition that if a pandiagonal square be cut into two pieces along a line between any two rows or

* Squares of this type were mentioned by P. De la Hire, J. Sauveur, and Euler. Attention was again called to them by A. H. Frost in the Quarterly Journal of Mathematics, London, 1878 , vol. $\mathrm{x} \vee$, pp. 34-49, and subsequently their properties have been discussed by several writers. Besides Frost's papers I have made considerable use of a paper by $\mathrm{E}$. McClintock in the American Journal of Mathematics, จol. xIx, 1897, pp. 99-120. 
any two columns, and the two pieces be interchanged, the new square so formed will be also pandiagonally magic. Hence it is obvious that by one vertical and one horizontal transposition of this kind any number can be made to occupy any specified cell.

Pandiagonal magic squares of an odd order can be constructed by a rule somewhat analogous to that given by De la Loubère, and described above. I proceed to give an outline of the method.

If we write the numbers in the scale of notation whose radix is $n$, with the understanding that the unit-digits run from 1 to $n$, it is evident, as in the corresponding explanation of why De la Loubère's rule gives a magic square, that all we have to do is to ensure that each row, column, and diagonal (whether broken or not) shall contain one and only one of each of the unit-digits, as also one and only one of each of the radix-digits.

\begin{tabular}{|c|c|c|c|c|}
\hline 7 & 20 & 3 & 11 & 24 \\
\hline 13 & 21 & 9 & 17 & 5 \\
\hline 19 & 2 & 15 & 23 & 6 \\
\hline 25 & 8 & 16 & 4 & 12 \\
\hline 1 & 14 & 22 & 10 & 18 \\
\hline
\end{tabular}

Figure xvi.

\begin{tabular}{|c|c|c|c|c|}
\hline $5+2$ & $15+5$ & $0+3$ & $10+1$ & $20+4$ \\
\hline $10+3$ & $20+1$ & $5+4$ & $15+2$ & $0+5$ \\
\hline $15+4$ & $0+2$ & $10+5$ & $20+3$ & $5+1$ \\
\hline $20+5$ & $5+3$ & $15+1$ & $0+4$ & $10+2$ \\
\hline $0+1$ & $10+4$ & $20+2$ & $5+5$ & $15+3$ \\
\hline
\end{tabular}

Figure zvii. Pandiagonal Square, $n=5$.

This is seen to be the case in the square of the fifth order delineated above in figures xvi and xvii.

Let us suppose that we write the numbers consecutively, and proceed from cell to cell by steps, using the term step $(a, b)$ to denote going $a$ cells to the right and $b$ cells up. Thus a step $(a, b)$ will take us from any cell to the $a$ th column to the right of it, to the $b$ th row above it, to the $(b+a)$ th diagonal above it sloping down to the right, and to the $(b-a)$ th diagonal above it sloping down to the left. In all cases we have the convention, as in De la Loubère's rule, that the movements along lines are taken cyclically; thus a step $n+a$ is equivalent to a step $a$. Of course, also, if $a$ means going $a$ cells to the right, then $-a$ will mean going $a$ cells to the left; thus if the 
bth upper line is outside the square we take it as equivalent to the $(n-b)$ th lower line.

It is clear that $a$ and $b$ cannot be zero, and that if $a$ and $b$ are prime to $n$ we can make $n-1$ steps from any cell from which we start, before we come to a cell already occupied. Thus the first $n$ numbers form a path which will give a different unit-digit in every row, column, and in one set of $n$ diagonals; of the other diagonals, $n-1$ are empty, and one contains every unit-digit-thus they are constructed on magical lines. We must take some other step $(h, k)$ from the cell $n$ to get to an unoccupied cell in which we place the number $n+1$. Continuing the process with $n-1$ more steps $(a, b)$ we get another series of $n$ numbers in various cells. If $h$ and $k$ are properly selected this second series will not interfere with the first series, and the rows, columns, and diagonals, as thus built up, will continue to be constructed on magical lines provided $h$ and $k$ are chosen so that the same unit-digit does not appear more than once in any row, column, and diagonal. We will suppose that this can be done, and that another cross-step $(h, k)$ of the same form as before enables us to continue filling in the numbers in compliance with the conditions, and that this process can be continued until the square is filled. If this is possible, the whole process will consist of $n$ series of $n$ steps, each series consisting of $n-1$ uniform steps $(a, b)$ followed by one cross-step $(h, k)$. The numbers inscribed after the $n$ cross-steps will be $n+1 ., 2 n+1,3 n+1, \ldots$, and these will be themselves connected by uniform steps $(u, v)$, where $u=(n-1) a+h \equiv k-a$, mod. $n$, and $v=(n-1) b+h \equiv k-b$. De la Loubère's rule is equivalent to taking steps $a=-1, b=1$, and cross-steps $h=1, k=0$.

I proceed to investigate the conditions that $a, b, h$, and $k$ must satisfy in order that the square can be constructed as above described with uniform steps $(a, b)$ and $(h, k)$. We notice at once that in order to secure the magic property in the rows and columns, we must have $a$ and $b$ prime to $n$; and to secure it in the diagonals, we must have $a$ and $b$ unequal and $b+a$ and $b-a$ prime to $n$. The leading numbers of the $n$ sequences of $n$ numbers, namely $1, n+1,2 n+1, \ldots$, are connected by steps 
$(u, v)$, where $u \equiv h-a$ and $v \equiv k-b$. Hence, if these are to fit in their places, we must also have $u$ and $v$ unequal, and $u, v, u+v$, and $u-v$ prime to $n$. Also $a, b, u$, and $v$ cannot be zero. Lastly the cross-steps $(h, k)$ must be so chosen that in no case shall a cross-step lead to a cell already occupied. This would happen, and therefore the rule would fail, if $p$ steps $(a, b)$ from any cell and $q$ steps $(u, v)$ from it, where $p$ and $q$ are each less than $n$, should lead to the same cell. Thus, to the modulus $n$, we cannot have $p a \equiv q u \equiv q(h-a)$, and at the same time $p b \equiv q v \equiv q(k-b)$.

It is impossible to satisfy these conditions if $n$ is equal to 3 or to a multiple of 3. For $a$ and $b$ are to be unequal, not zero, and less than $n$, and $a+b$ is to be less than $n$ and prime to $n$. Thus we cannot construct a pandiagonal square of the third order.

Next I will show that, if $n$ is not a multiple of 3 , these conditions are satisfied when $a=1, b=2, h=0, k=-1$, and therefore that in this case these values provide a particular solution of the general problem. It is at once obvious that in this case $a$ and $b$ are unequal, not zero, and prime to $n$, that $b+a$ and $b-a$ are prime to $n$, and that the corresponding relations for $u$ and $v$ are true. The remaining condition for the validity of a rule based on these particular steps is that it shall be impossible to find integral values of $p$ and $q$ each less than $n$, which will simultaneously make $p \equiv-q$, and $2 p \equiv-3 q$. This condition is satisfied. Hence, any odd pandiagonal square of an order which is not a multiple of 3 can be constructed by this rule. Thus, to form a pandiagonal square of the fifth order we may put 1 in any cell; proceed by four successive steps, like a knight's move, of one cell to the right and two cells up, writing consecutively numbers $2,3,4,5$ in each cell, until we come to a cell already occupied; then take one step, like a rook's move, one cell down, and so on until the square is filled. This is illustrated by the square delineated in figure $x v i$.

Further discussion of the general case depends on whether or not $n$ is prime; here I will confine myself to the simpler alternative, and assume that $n$ is prime: this will sufficiently 
illustrate the theory. From the above relations it follows that we cannot have $p q a(k-b) \equiv p q b(h-a)$, that is, $p q(a k-b h) \equiv 0$. Therefore $a k-b h$ cannot be a multiple of $n$, that is, it must be prime to $n$. If this condition is fulfilled, as well as the other conditions given above, each cross-step $(h, k)$ can be made in due sequence, and the square can be constructed. The result that $a k-b h$ is prime to $n$ shows that the cross-step $(h, k)$ must be chosen so as to take us to an unoccupied cell not in the same row, column, or diagonal (broken or not) as the initial number. By noting this fact we can in general place any two given numbers in two assigned cells.

There are some advantages in having the cross-steps uniform with the other steps, since, as we shall see later, the square can then be written in a form symmetrical about the centre. This will be effected if we take $h=-b, k=a$. If $n$ is prime our conditions are then satisfied if $b$ be any number from 2 to $(n-1) / 2$, if $a$ be positive and less than $b$, and if $a^{2}+b^{2}$ be prime to $n$. We can, if we prefer, take $h=b, k=-a$; but it is not possible to take $h=a$ and $k=-b$, or $h=-a$ and $k=b$, since they make $u=0$ or $v=0$.

For instance, if we use a knight's move, we may take $a=1$, $b=2$. The square of the seventh order given below (figure xviii)

\begin{tabular}{|c|c|c|c|c|c|c|}
\hline 35 & 23 & 18 & 13 & 1 & 45 & 40 \\
\hline 4 & 48 & 36 & 31 & 26 & 21 & 9 \\
\hline 22 & 17 & 12 & 7 & 44 & 39 & 34 \\
\hline 47 & 42 & 30 & 25 & 20 & 8 & 3 \\
\hline 16 & 11 & 6 & 43 & 38 & 33 & 28 \\
\hline 41 & 29 & 24 & 19 & 14 & 2 & 46 \\
\hline 10 & 5 & 49 & 37 & 32 & 27 & 15 \\
\hline
\end{tabular}

Figure rviii. A Pandiagonal Symmetrical Square, $n=7$.

is constructed by this rule. But in the case of a square of the fifth order we cannot use a knight's move, since, if $a=1$, and $b=2$, we have $a^{2}+b^{2}=5$. Hence the use of a knight's move is not applicable when $n$ is 5 , or a multiple of 5 . 
The construction of singly-even pandiagonal squares (that is, those whose order is $4 m+2$ ) is impossible, but that of doublyeven squares (that is, those whose order is $4 \mathrm{~m}$ ) is possible.

Here is one way of constructing a doubly-even pandiagonal square. Suppose the order of the square is $4 m$, and as before let us write the number in a cell in the scale $4 m$, that is, as $4 m p+r$, so that $p$ and $r$ are the radix and unit-digits, with the convention that $r$ cannot be zero. Place $p_{1}, p_{2}, p_{8}, \ldots, p_{4 m}$ in order in the cells in the bottom row. Proceeding from $p_{1}$ by steps $(2 m, 1)$ fill up $2 m$ cells with it. And proceed similarly with the other radix-digits. Next place $r_{1}, r_{2}, \ldots, r_{4 m}$ in order in the cells in the first column. Proceeding from $r_{1}$ by steps $(1,2 m)$ fill up $2 m$ cells with it. And proceed similarly with the other unit-digits. Then if we take for $r_{1}, r_{2}, \ldots, r_{4 m}$, the values $1,2, \ldots, 2 m, 4 m, 4 m-1, \ldots, 2 m+1$, and for $p_{1}, p_{2}, \ldots, p_{4 m}$, the values $0,1, \ldots, 2 m-1,4 m-1, \ldots, 2 m$, the square will be pandiagonally magic. I leave the demonstration to my readers. In the case when $m=1, n=4$, the $p$ and $r$ subsidiary squares, and the resulting magic square are shown in figures $x i x, x z$, xxi, ii.

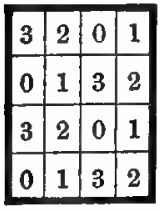

Subsidiary p Square. Subsidiary r Square. Figure xix.

\begin{tabular}{|l|l|l|l|}
\hline 3 & 2 & 3 & 2 \\
\hline 4 & 1 & 4 & 1 \\
\hline 2 & 3 & 2 & 3 \\
\hline 1 & 4 & 1 & 4 \\
\hline
\end{tabular}

Figure $\mathbf{x x}$.

\begin{tabular}{|r|r|r|r|}
\hline $12+3$ & $8+2$ & $0+3$ & $4+2$ \\
\hline $0+4$ & $4+1$ & $12+4$ & $8+1$ \\
\hline $12+2$ & $8+3$ & $0+2$ & $4+3$ \\
\hline $0+1$ & $4+4$ & $12+1$ & $8+4$ \\
\hline
\end{tabular}

Resulting Square.

Figure xxi.

The rows, columns, and all diagonals of pandiagonal squares possess the magic property. So also do a group of any $n$ numbers connected cyclically by steps $(c, d)$ provided the first two numbers of the group are such that when divided by $n$ they have either different quotients or different remainders. Such groups include rows, columns, and diagonals as particular cases. Thus in the square delineated in figure ii on page 137 the numbers 1, 7, 10, 16 form a magic group whose sum is 34, connected cyclically by steps $(1,3)$. Again in the square delineated in figure xviii above, 
$10,30,1,28,48,19,39$ form a magic group connected cyclically by steps $(2,3)$.

Symmetrical Squares. It has been suggested that we might impose on the construction of a magic square of the order $n$ the condition that the sum of any two numbers in cells skewly related to one another shall be constant and equal to $n^{2}+1$. Such squares are called Symmetrical.

The construction of odd symmetrical squares of the order $n$, when $n$ is prime to 3 and 5 , involves no difficulty. We can begin by placing the mean number in the middle cell and work from that, either in both directions or forwards, making the number 1 follow after $n^{2}$; we can also effect the same result by constructing a pandiagonal square of the order $n$ and then transposing a certain number of rows and columns. If the rule given above on page 154 , where $a=1, b=2, h=2, k=-1$, be followed, this will lead to placing the number 1 in the $(n+3) / 2$ th cell of the top row, as is exemplified in figure xviii.

Such a square must be symmetrical, for if we begin with the middle number $\left(n^{2}+1\right) / 2$, which I will denote by $m$, in the middle cell, and work from it forwards with the numbers $m+1$, $m+2, \ldots$, and backwards with the numbers $m-1, m-2, \ldots$, the pairs of cells filled by the numbers $m+1$ and $m-1, m+2$ and $m-2$, \&c., are necessarily skewly related, and the sum of the numbers in each pair is $2 m$. This was first pointed out by McClintock.

The construction of doubly-even symmetrical pandiagonal squares is also possible, but the analysis is too lengthy for me to find room here for it.

In a symmetrical square any $n$ such pairs of numbers together with the number in the middle cell will form a magic group. For instance in figure xviii, the group $32,18,36,14,47,3$, 25 is magic. So also is the group $47,3,35,15,13,37,25$. Thus in a symmetrical pandiagonal square, even of a comparatively low order, there are hundreds of magic groups of $n$ numbers whose sum is constant.

Doubly-Magic Squares. In another species of hyper-magic squares the problem is to construct a magic square of the $n$th 
order in such a way that if the number in each cell is replaced by its square the resulting square shall also be magic*. Here are two examples. Figure xxii represents a magic square of the eighth order (the sum of the numbers in each line being

\begin{tabular}{|c|c|c|c|c|c|c|c|}
\hline 5 & 31 & 35 & 60 & 57 & 34 & 9 & 30 \\
\hline 19 & 9 & 53 & 46 & 47 & 56 & 18 & 12 \\
\hline 16 & 22 & 42 & 39 & 52 & 61 & 27 & 1 \\
\hline 63 & 37 & 25 & 24 & 3 & 14 & 44 & 50 \\
\hline 26 & 4 & 64 & 49 & 38 & 43 & 13 & 23 \\
\hline 11 & 51 & 15 & 2 & 21 & 28 & 62 & 40 \\
\hline 54 & 48 & 20 & 11 & 10 & 17 & 55 & 45 \\
\hline 36 & 58 & 6 & 29 & 32 & 7 & 33 & 59 \\
\hline
\end{tabular}

Figure xxii. A Doubly-Magic Square, $n=8$.

\begin{tabular}{|c|c|c|c|c|c|c|c|c|}
\hline $2 n+8$ & $7 n+2$ & $3 n+5$ & $6 n+9$ & $5 n+3$ & $n+6$ & $4 n+7$ & $0+1$ & $8 n+4$ \\
\hline $6 n+7$ & $5 n+1$ & $n+4$ & $4 n+8$ & $0+2$ & $8 n+5$ & $2 n+9$ & $7 n+3$ & $3 n+6$ \\
\hline $4 n+9$ & $0+3$ & $8 n+6$ & $2 n+7$ & $7 n+1$ & $3 n+4$ & $6 n+8$ & $5 n+2$ & $n+5$ \\
\hline $3 n+2$ & $2 n+5$ & $7 n+8$ & $n+3$ & $6 n+6$ & $5 n+9$ & $8 n+1$ & $4 n+4$ & $0+7$ \\
\hline$n+1$ & $6 n+4$ & $5 n+7$ & $8 n+2$ & $4 n+5$ & $0+8$ & $3 n+3$ & $2 n+6$ & $7 n+9$ \\
\hline $8 n+3$ & $4 n+6$ & $0+9$ & $3 n+1$ & $2 n+4$ & $7 n+7$ & $n+2$ & $6 n+5$ & $5 n+8$ \\
\hline $7 n+5$ & $3 n+8$ & $2 n+2$ & $5 n+6$ & $n+9$ & $6 n+3$ & $0+4$ & $8 n+7$ & $4 n+1$ \\
\hline $5 n+4$ & $n+7$ & $6 n+1$ & $0+5$ & $8 n+8$ & $4 n+2$ & $7 n+6$ & $3 n+9$ & $2 n+3$ \\
\hline $0+6$ & $8 n+9$ & $4 n+3$ & $7 n+4$ & $3 n+7$ & $2 n+1$ & $5 n+5$ & $n+8$ & $6 n+2$ \\
\hline
\end{tabular}

Figure zxiii. A Doubly-Magic Square, $n=9$.

equal to 260) so constructed that if the number in each cell is replaced by its square the resulting square is also magic (the sum of the numbers in each line being equal to 11180). Figure xxiii, where $n$ stands for 9 , represents a doubly-magic square of

* See M. Coccoz in L'Illustration, May 29, 1897. The subject has been studied by Messieurs G. Tarry, B. Portier, M. Coccoz, A. Rilly, E. Barbette and W. S. Andrews. More than 200 such squares have been given by Rilly in his Ĺtudes sur les Triangles et les Carrés Magiques aux deux premiers degrés, Trojes, 1901. 
the ninth order: it is written in a form which shows it as formed by the superposition of two magic squares, one made with the unit-digits and the other with the radix-digits.

Trebly-Magic Squares. The construction of squares which shall be magic for the original numbers, for their squares, and for their cubes has also been studied. I know of no square of this kind which is of a lower order than 128 , and the construction of a square of that order is not a "recreation."

Other Magic Problems. Other problems, closely related to magic squares, will suggest themselves; the following will serve as specimens.

Magic Card Square*. The first of these is the familiar problem of placing the sixteen court cards (taken out of a pack) in the form of a square so that no row, no column, and neither of the diagonals shall contain more than one card of each suit and one card of each rank. The solution presents no difficulty, and is indicated in figure $\mathrm{xxv}$ below. There are 72 fundamental solutions, each of which by reflexions and reversals produces 7 others.

Euler's Officers Problemt. A similar problem, proposed by Euler in 1779, consists in arranging, if it be possible, thirty-six officers taken from six regiments-the officers being in six groups, each consisting of six officers of equal rank, one drawn from each regiment; say officers of rank, $a, b, c, d, e, f$, drawn from the 1st, $2 \mathrm{nd}$, 3rd, 4th, 5th, and 6th regiments-in a solid square formation of six by six, so that each row and each file shall contain one and only one officer of each rank and one and only one officer from each regiment. The problem is insoluble. Extension of Euler's Problem. More generally we may investigate the arrangement on a chess-board, containing $n^{2}$

* Ozanam, 1723 edition, vol. Iv, p. 434.

$†$ Euler's Commentationes Arithmeticae, Petrograd, 1849, vol. II, pp. 302361. See also a paper by G. Tarry in the Comptes rendus of the French Association for the Advancement of Science, Paris, 1900, vol. II, pp. 170-203; and various notes in L'Intermédiaire des Mathématiciens, Paris, vol. III, 1896, pp. 17,90 ; vol. v, 1898, pp. 83 , 176, 252 ; vol. vi, 1899, p. 251 ; vol. vII, 1900, pp. 14, 311 . 
cells, of $n^{2}$ counters (the counters being divided into $n$ groups, each group consisting of $n$ counters of the same colour and numbered consecutively $1,2, \ldots, n$ ), so that each row and each column shall contain no two counters of the same colour or marked with the same number. Such arrangements are termed Eulerian Squares.

For instance, if $n=3$, with three red counters $a_{1}, a_{2}, a_{3}$, three white counters $b_{1}, b_{2}, b_{3}$, and three black counters $c_{1}, c_{2}, c_{3}$, we can satisfy the conditions by arranging them as in figure xxiv below. If $n=4$, then with counters $a_{1}, a_{2}, a_{3}, a_{4} ; b_{1}, b_{2}, b_{3}, b_{4}$; $c_{1}, c_{2}, c_{3}, c_{4} ; d_{1}, d_{2}, d_{3}, d_{4}$, we can arrange them as in figure $\mathrm{xxv}$ below. A solution when $n=5$ is shown in figure xxvi.

\begin{tabular}{|l|l|l|}
\hline$a_{1}$ & $b_{2}$ & $c_{3}$ \\
\hline$b_{3}$ & $c_{2}$ & $a_{2}$ \\
\hline$c_{2}$ & $a_{8}$ & $b_{1}$ \\
\hline
\end{tabular}

Figure xxiv.

\begin{tabular}{|l|l|l|l|}
\hline$a_{1}$ & $b_{2}$ & $c_{3}$ & $d_{4}$ \\
\hline$c_{4}$ & $d_{8}$ & $a_{2}$ & $b_{1}$ \\
\hline$d_{2}$ & $c_{1}$ & $b_{4}$ & $a_{3}$ \\
\hline$b_{3}$ & $a_{4}$ & $d_{1}$ & $c_{2}$ \\
\hline
\end{tabular}

Figure $\mathbf{x x \nabla}$.

\begin{tabular}{|c|c|c|c|c|}
\hline$a_{1}$ & $b_{2}$ & $c_{3}$ & $d_{4}$ & $e_{5}$ \\
\hline$d_{3}$ & $e_{4}$ & $a_{5}$ & $b_{1}$ & $c_{2}$ \\
\hline$b_{6}$ & $c_{1}$ & $d_{2}$ & $e_{3}$ & $a_{4}$ \\
\hline$e_{2}$ & $a_{3}$ & $b_{4}$ & $c_{5}$ & $d_{1}$ \\
\hline$c_{4}$ & $d_{5}$ & $e_{1}$ & $a_{2}$ & $b_{3}$ \\
\hline
\end{tabular}

Figure xxvi.

The problem is soluble if $n$ is odd; it would seem* that it is insoluble if $n$ is of the form $2(2 m+1)$. If solutions when $n=a$ and when $n=b$ are known, a solution when $n=a b$ can be written down at once. If $n$ is an odd prime greater than 3, a solution can be given which covers the diagonals as well as the rows and columns of the square. The theory is closely connected with that of magic squares.

Magic Domino Squares. Magic problems can be made with dominoes. An ordinary set of dominoes, ranging from double zero to double six, contains 28 dominoes. Each domino is a rectangle formed by fixing two small square blocks together side by side: of these 56 blocks, eight are blank, on each of eight of them is one pip, on each of another eight of them are two pips, and so on. It is required to arrange the dominoes so that the 56 blocks form a square of 7 by 7 bordered by one line

* C. Planck, The Monist, Chicago, vol. xxIx, 1919, p. 308. 
of 7 blank squares and so that the sum of the pips in each row, column, and diagonal of the square is equal to 24. A solution* is given below.

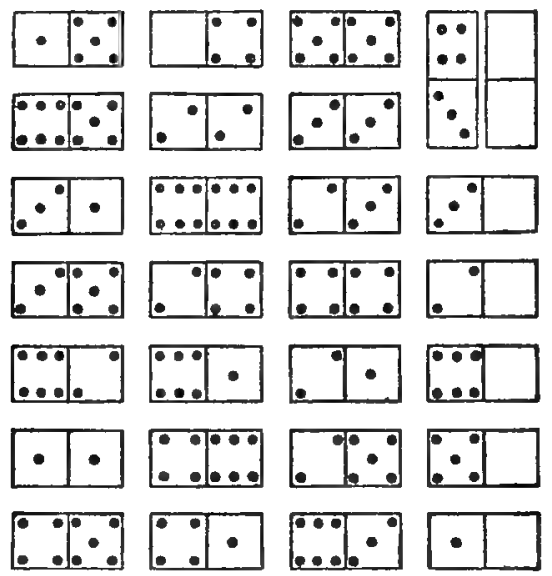

Figure sxrii. Magic Domino Square.

If we select certain dominoes out of the set and reject the others we can use them to make various magic puzzles. As instance, I give in figure xxviii two examples of magic squares of this kind due respectively to Escott and Dudeney.
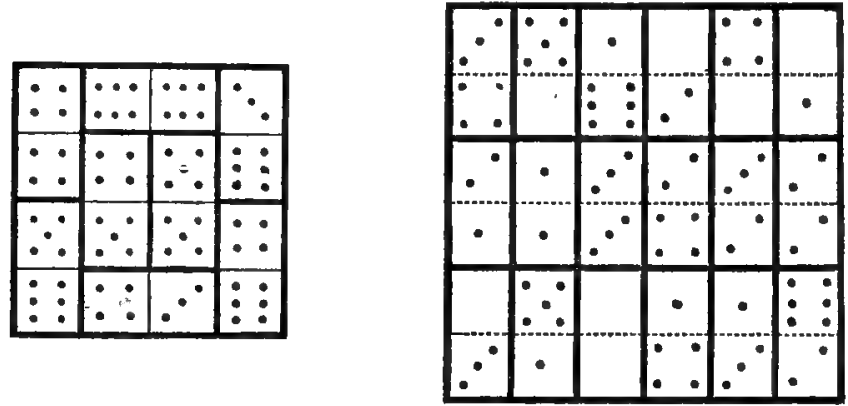

Figure xxvili. Magic Domino Squares.

Magic Coin Squares. There are somewhat similar questions concerned with coins or postage stamps. Here is one applicable

- See L'Illustration, July 10, 1897. 
to a square of the third order divided into nine cells. If a fiveshilling piece is placed in the middle cell $c_{1}$ and a florin in the cell below it, namely, in $a_{8}$, it is required to place the fewest possible current English coins in the remaining seven cells so that in each cell there is at least one coin, so that the total value of the coins in every cell is different, and so that the sum of the values of the coins in each row, column, and diagonal is fifteen shillings: it will be found that thirteen additional coins will suffice. Such problems or puzzles involve little or no knowledge of mathematics, and need no further mention here.

\section{ADDENDUM.}

Note. Page 161. Magic Coin Squares. The solution of the problem enunciated in the test is as follows. Taking the notation of figure xxiv we must put a double-florin and a sixpence in cell $a_{1}$, two double-florins in cell $b_{2}$, a half-crown in cell $c_{3}$, a florin and a shilling in cell $b_{3}$, a crown and a florin in cell $a_{2}$, a crown and a half-crown in cell $c_{2}$, a crown and a sixpence in cell $b_{1}$ : see The Strand Magazine, London, December, 1896, pp. $720,721$. 


\section{CHAPTER VIII.}

\section{BEES AND THEIR CELLS.}

The general form of the honey cells of bees has long been known. Early in the eighteenth century it was suggested that these cells were shaped by the bees so as to use their available wax in the most economical manner, and Samuel Koenig, a young Swiss mathematician of some repute, was asked to look into the problem. He said that the suggestion was true, and added that the bees had thus solved a question beyond the power of mathematicians unacquainted with the calculus. In fact the bees do not use their wax in the most economical way, and the problem of determining rhomboidal ends of a hexagonal prismatic cell of given base and volume so that the surface is a minimum is easily soluble by classical geometry; but Koenig's ponderous joke about the ability of the bees has been repeated so often that it seems worth while to give the history of the subject.

A honey comb, taken from a bee hive under normal conditions, is in the shape of a thick slab with two parallel faces containing between them cells made of wax and filled with honey. The open bases of these cells are on the faces of the slabs and are regular hexagons. If the faces of the slab are carefully drawn apart, not an easy task, the cells will be found to be arranged in two systems, each system resting on one of the faces, and so placed that the top of each cell fits into the space formed by the tops of three adjacent cells of the other system, its axis being the continuation of the line of junction of these three cells. Each cell is a prism, whose six sides (each perpendicular to the hexagonal base) are trapeziums, and whose top is 
pointed and formed by three equal rhombs, the angles at the apex being obtuse. So much probably has been familiar as long as bees have been kept and their habits noted.

As a result of investigations* made in the eighteenth century, we may go further, and say that the cells are built on a uniform plan, though they vary slightly among themselves. In a normal comb the height of the longest side of one of the trapeziums is a little more than four times the radius of the incircle of the hexagonal base; the angle between every pair of adjacent planes is $120^{\circ}$; and each acute angle of every rhomb and trapezium is about $70^{\circ}$, the obtuse angles being of course supplementary to this. Some writers on natural history are more definite, and say that each such acute angle is $70^{\circ} 31^{\prime} 44^{\prime \prime}$. We may take this as the typical value, though the edges of a cell are not sharply defined, and these angles cannot be measured to this accuracy.

It happens that for these angular values a cell of this form with a given volume and base has the smallest surface, or in other words gives the maximum storage space which can be provided with a given amount of wax. As soon as this was discovered, it was only natural that, taking into account the conditions under which bees live and work, it should be conjectured that they use the available wax in the most economical manner, and whether by reasoning, instinct, or some other process have arrived at a result which certainly is far from obvious.

The story of this "economy conjecture" starts with a memoir presented to the French Academy in 1712 by G. F. Maraldi. In this he said that, as nearly as he could measure them, each of the smaller angles of the rhombs and trapeziums was $70^{\circ}$, each of the larger angles being of course the supplement of this. Naturally it occurred to him to investigate the problem of the values of these angles on the supposition that those of the rhombs and trapeziums were exactly equal and, on this hypothesis, he found that their values were $70^{\circ} 32^{\prime}$ and its supplement: a result consistent with his measurements. The imposition of the con-

* For references, see J. W. L. Glaisher, Philosophical Magazine, London, 1873, fourth series, vol. xIVI, pp. 103-122. 
dition that the acute angle of the rhomb is equal to the acute angle of the trapezium ensures that the surface of the cell is a minimum, but Maraldi did not suspect this.

Some years later Réaumur took up the problem. Conjecturing that the shape of the rhombs might arise from the desire of the bees to economise wax, he asked Koenig, who was then staying in Paris, to determine the angles of the rhombs so that for a given capacity the surface of a cell of this form should be a minimum. The problem presents no difficulty*, and Koenig gave as the result $70^{\circ} 34^{\prime}$ and $109^{\circ} 26^{\prime}$, which are wrong by about $2^{\prime}$. $\mathrm{He}$ further stated, also inaccurately, the amount of wax saved by using this form of top instead of a plane one, making the natural assumption that the walls of the cells are of uniform thickness. Koenig used the calculus in his work; he said this was necessary for the purpose, and jokingly added, as stated above, that the bees had in fact solved a problem beyond the powers of the old geometricians. His answer was not worthy of his reputation; his numerical mistakes showed carelessness, his statement about the old geometry was untrue, and his joke was at least unlucky, for some writers, whose sense of humour was not highly developed, took it literally. The volume containing Réaumur's researches in which Koenig's conclusions are embodied was published in 1740. Réaumur's investigations on bee life are classical and were continued by F. Huber, J. H. Fabre, and M. Maeterlinck, but with this side of the subject I am not here concerned.

Maclaurin came across Koenig's statements, and in 1743 published a geometrical solution of the problem, from which he correctly deduced the values of the angles as $70^{\circ} 31^{\prime} 44^{\prime \prime}$ and its supplement. He pointed out Koenig's numerical blunders, and

* Let $a$ be the length of one of the hexagonal edges, which is also the base of one of the trapezium sides, $h$ the longest side of the trapezium, $A$ the area of the hexagonal base, and $\theta$ the inclination of one of the rhombs to the hexagonal base. Then the volume of the cell is equal to $h A$ and is independent of $\theta$. The surface of the cell is

$$
3 a(4 h-a \tan \theta+a \sqrt{ } 3 \sec \theta) / 2 .
$$

If this is a minimum, we have $\sin ^{2} \theta=1 / 3$. And the acute angle of the rhombus $=2 \theta=\arccos (1 / 3)$, or to the nearest second $70^{\circ} 31^{\prime} 44^{\prime \prime}$. 
stated that the saving of wax by using rhomboidal ends instead of a plane hexagonal top was almost one-quarter of the wax required for a plane hexagonal end: the actual fraction is $(\sqrt{ } 3-\sqrt{ } 2) / \sqrt{ } 3$.

Boscovich also had his attention called to the problem, and he gave two solutions, published in 1760 , one by pure geometry and the other by the calculus. In these he pointed out the numerical slip made by Koenig, the extreme difficulty of making accurate measurements of actual cells, and the closeness to the typical form given by Maraldi. In his calculation of the amount of wax saved there is a misprint, but the error is obvious to any careful reader. He was the first to point out that the angle between every two adjacent planes is $120^{\circ}$.

In 1781 Lhulier gave another geometrical solution of the problem, and treated the obvious extension of determining the best ratio of the depth of the cell to the width of the base. $\mathrm{He}$ also discussed the saving of wax obtained by using cells of different forms, and came to the conclusion, now generally accepted, that economy in using wax cannot have been the main reason for the form of cell adopted.

Most people are interested in bee life, but the economy conjecture about cell construction presents no mathematical difficulty, and probably the papers by Maclaurin and Boscovich would not have been written had not Koenig stated that the question was insoluble by classical geometry. Their papers with that of Lhulier completely solved the problem as presented to them. Unluckily, Lord Brougham in the nineteenth century described it afresh, and in doing so made certain inaccurate statements. These were pointed out by Leslie Ellis and Glaisher, but Brougham's account had a wide circulation, and his mistakes have been copied by some modern popular writers.

Brougham, however, brought out the fact, previously noted but perhaps not widely known, that the bees usually make the walls of the rhombs and adjoining parts thicker than the walls of the rest of the sides. This disposes finally of the conjecture that the form of cell adopted is that which is most economical of wax, for that hypothesis rests on the assumption that the walls of 
the cells are of uniform thickness. There are however other objections to the conjecture, since it deals only with cells of a very special shape, and neglects all consideration of their use for purposes other than the storage of honey. It is reasonable to hold that the form of cell adopted is on the whole that which best suits the bees, but saving of wax cannot be the chief reason for it.

Another vague but more plausible explanation of the form of the cells is that their shape and size depend largely on the manner in which the bees can best use the organs of their bodies and senses. One of the most striking features of the cells is the fact that the angle between every adjacent pair of planes is $120^{\circ}$, and perhaps this is an essential characteristic. Leslie Ellis* in an interesting essay suggested that the explanation of this may be found in the fact that bees, besides their composite eyes, "have three single eyes placed lower down, and "probably serving for the vision of near objects. Assume that "the axes of these eyes diverge so as to be respectively normal "to three ideal planes forming a solid angle, each dihedral angle "of which is of 120 degrees. Geometry shows that every solid "angle of the bee's cell is precisely similar to this type, so that "a bee looking at it with his three single eyes, might have "direct vision with each eye of one of the three planes of the "solid angle. This direct vision may correspond to a particular "sensation, so that a bee is not satisfied till it is attained." This is ingenious: it is too artificial to make me think it probable, but it is worth mentioning, for Leslie Ellis was an acute observer.

Another proffered explanation of the form of the cells rests on the assumption that, when forming the comb from a slab of wax, the bees work in as dense and regular a formation as is possible. It will be observed that the upper portion of a typical cell is half a rhombic dodecahedron: a fact noted by Leslie Ellis, who gave the following construction for making a model of a typical cell. Take two equal cubes. Divide one of them into six pyramids, the apex of each being at the

- R. Leslie Ellis, Mathematical...Writings, Cambridge, 1863, pp. 353-357. 
centre and its base a face of the cube. Place these pyramids each on one face of the other cube. The resulting solid is a dodecahedron with rhombic faces, 14 vertices, and 24, edges; space can be filled with such solids. If such a dodecahedron is bisected by a plane through the centre and perpendicular to a diagonal of the cube, either half is of the form of the typical bee's cell except that its depth is less than is usual in a honey comb.

Now the following argument* shows that rhombic dodecahedrons are cell shapes naturally formed under certain physical conditions. Consider a large space filled with as many equal spheres as is possiblet, which are crushed together symmetrically till all the space between them is occupied; such an initial arrangement is a natural one because it is of maximum density and maximum stability. Initially each sphere touches twelve adjacent spheres at the mid-points of the twelve edges of a cube, its intermediate portions bulging out through the six faces of the cube. When the spheres are crushed together, these twelve points of contact move inwards along the radii, and the six intermediate portions are squeezed into the over-arching spaces which lie between the points of contact of the surrounding spheres. Since there are four spheres round every face of the cube, these portions will be squeezed into four-sided pyramids, the faces of each being coterminous with those of the adjacent pyramid, and both being the ultimate position of the original

* S. Bryant, London Mathematical Society Proceedings, 1884-85, vol. х¥1, pp. $311-315$.

† A large box can be filled with a number of small equal spheres arranged in horizontal layers, one on top of the other, in various ways. It might be filled so that each sphere rests on the top of the sphere immediately below it in the next layer, touches each of 4 adjacent spheres in the same layer, and touches one sphere in the layer above it, thus each sphere is in contact with 6 others: such an arrangement gives the smallest number of spheres with which the box can be filled. We might also fill the box with spheres arranged so that each of them is in contact with 2 spheres in the next lower layer, with 4 in the same layer, and with 2 in the next higher layer. Or we might fill the box with spheres arranged so that each of them is in contact with 4 spheres in the next lower layer, with 4 in the same layer, and with 4 in the next higher layer. Such an arrangement occurs if the box be shaken steadily, and thus it is the most stable arrangement; it gives the greatest number of spheres with which the box can be filled. In this arrangement each sphere is in contact with 12 others. 
plane of contact. Each of these faces makes half a right angle with the face of the cube, since the sum of two of them is supplementary to the angle between two adjacent faces of the cube. Hence, in the final position, we have the twelve points of contact represented by the mid-points of the edges of a smaller cube, and the intermediate portions heaped up into six square pyramids on the faces of the cube, the faces of the pyramids making an angle of $45^{\circ}$ with those of the cube. Thus the form generated is a rhombic dodecahedron.

Reversing the process, it follows that if a homogeneous solid has equally efficient centres of excavation distributed uniformly through it, and excavation goes on till the walls of the cells produced are of uniform thickness, we shall finally arrive at a system of rhombic dodecahedrons filling the space excavated.

Applying this theory to the construction of the honey comb, Mrs Bryant states the following hypothesis as to why the bees construct their cells in the form they do. "We might," says she, "reasonably expect that the bees, who are the cell-excavators, "should by natural instinct distribute themselves as densely as "possible, and with a considerable degree of regularity, and that "their activities should be equal and symmetrical about the "working parts of their bodies. The facts confirm this reason"able expectation. The bees distribute themselves, with "apparent uniformity, at the two sides of a homogeneous cake "of wax which has been previously deposited. In it they "excavate cells, at doubtless uniform rates of work, and continue "excavating till their work is as complete as possible, and the "walls of the cells therefore of uniform thickness. Meanwhile, "the excavated wax is used to build up higher the open cell "walls. Hence, the cells ought to be elongated rhombic semi"dodecahedra; and this is just what they are, the axis of the "cell corresponding to a diagonal of the primary cube, and the "apex being one of the trihedral vertices of the dodecahedron. "Each face at the apex fits exactly against one face of a cell in "the opposite system. Each cell, therefore, is in contact with "three cells of the opposite system."

"It follows, from this last mentioned fact, that the bees must 
"distribute themselves with maximum density, not only on each "side separately, but on the two considered jointly. This, as a "case of instinct, is certainly remarkable, but the possibilities "of trial and error are sufficient to account for it. It is not "unreasonable to expect that the bees should learn how to "employ the largest possible number of themselves on a piece "of wax to be excavated, this being a thing which they would "naturally try to do; though it would be strange, in comparison, "if they tried to effect those other two ends, of maximum "economy in wax, and maximum strength of structure, which, "as a matter of fact, they do effect." 
CHAPTER IX.

UNICURSAL PROBLEMS.

I propose to consider in this chapter some problems which arise out of the theory of unicursal curves. I shall commence with Euler's Problem and Theorems, and shall apply the results briefly to the theories of Mazes and Geometrical Trees. The reciprocal unicursal problem of the Hamilton Game will be discussed in the latter half of the chapter.

Euler's Problem. Euler's problem has its origin in a memoir* presented by him in 1736 to the St Petersburg Academy, in which he solved a question then under discussion as to whether it was possible from any point in the town of Königsberg to take a walk in such a way as to cross every bridge in it once and only once and return to the starting point.

The town is built near the mouth of the river Pregel, which there takes the form indicated below and includes the island of Kneiphof. In the eighteenth century there were (and according to Baedeker there are still) seven bridges in the positions shown in the diagram, and it is easily seen that with such an arrangement the problem is insoluble. Euler however did not confine himself to the case of Königsberg, but discussed the general problem of any number of islands connected in any way by bridges. It is evident that the question

" 'Solutio problematis ad Geometriam situs pertinentis,' Commentarii Academiae Scientiarum Petropolitanae for 1736, Petrograd, 1741, vol. vin, pp. 128-140. This has been translated into French by M. Ch. Henry; see Lucas, vol. I, part 2, pp. 21-33. 
will not be affected if we suppose the islands to diminish to points and the bridges to lengthen out. In this way wo

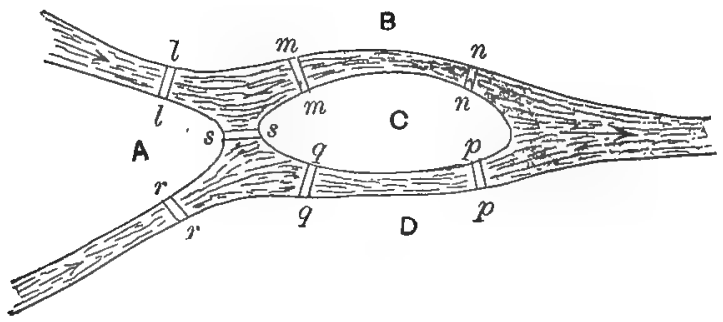

ultimately obtain a geometrical figure or network. In the Königsberg problem this figure is of the shape indicated below, the areas being represented by the points $A, B, C, D$, and the bridges being represented by the lines $l, m, n, p, q, r, s$.

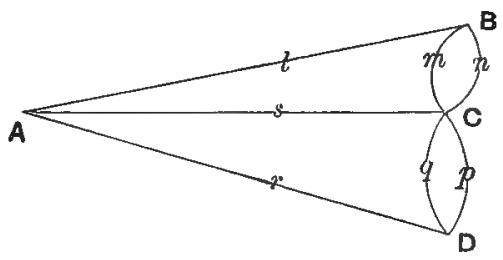

Euler's problem consists therefore in finding whether a given geometrical figure can be described by a point moving so as to traverse every line in it once and only once. A more general question is to determine how many strokes are necessary to describe such a figure so that no line is traversed twice: this is covered by the rules hereafter given. The figure may be either in three or in two dimensions, and it may be represented by lines, straight, curved, or tortuous, joining a number of given points, or a model may be constructed by taking a number of rods or pieces of string furnished at each end with a hook so as to allow of any number of them being connected together at one point.

The theory of such figures is included as a particular case 
in the propositions proved by Listing in his Topologie*. I shall, however, adopt here the methods of Euler, and I shall begin by giving some definitions, as it will enable me to put the argument in a more concise form.

A node (or isle) is a point to or from which lines are drawn. A branch (or bridge or path) is a line connecting two consecutive nodes. An end (or hook) is the point at each termination of a branch. The order of a node is the number of branches which meet at it. A node to which only one branch is drawn is a free node or a free end. A node at which an even number of branches meet is an even node: evidently the presence of a node of the second order is immaterial. A node at which an odd number of branches meet is an odd node. A figure is closed if it has no free end: such a figure is often called a closed network.

A route consists of a number of branches taken in consecutive order and so that no branch is traversed twice. A closed route terminates at a point from which it started. A figure is described unicursally when the whole of it is traversed in one route.

The following are Euler's results. (i) In a closed network the number of odd nodes is even. (ii) A figure which has no odd node can be described unicursally, in a re-entrant route, by a moving point which starts from any point on it. (iii) A figure which has two and only two odd nodes can be described unicursally by a moving point which starts from one of the odd nodes and finishes at the other. (iv) A figure which has more than two odd nodes cannot be described completely in one route; to which Listing added the corollary that a figure which has $2 n$ odd nodes, and no more, can be described completely in $n$ separate routes. I now proceed to prove these theorems.

* Die Studien, Göttingen, 1847, part x. STee also Tait on 'Listing's Topologie,' Philosophical Magazine, London, January, 1884, series 5, vol. xvII, pp. 30-46; and Collected Scientific Papers, Cambridge, vol. $\pi, 1900, \mathrm{pp}$. 85-98. The problem was discussed by J. O. Wilson in his Traversing of Geometrical Figures, Oxford, 1905. 
First. The number of odd nodes in a closed network is even.

Suppose the number of branches to be $b$. Therefore the number of hooks is $2 b$. Let $k_{n}$ be the number of nodes of the $n$th order. Since a node of the $n$th order is one at which $n$ branches meet, there are $n$ hooks there. Also since the figure is closed, $n$ cannot be less than 2 .

Hence

$$
\therefore 2 k_{8}+3 k_{8}+4 k_{4}+\ldots+n k_{n}+\ldots=2 b \text {. }
$$

\section{Henco}

$3 k_{8}+5 k_{b}+\ldots$ is even.

$$
\therefore k_{\mathrm{a}}+k_{\mathrm{s}}+\ldots \text { is even. }
$$

Second. A figure which has no odd node can be described unicursally in a re-entrant route.

Since the route is to be re-entrant it will make no difference where it commences. Suppose that we start from a node $A$. Every time our route takes us through a node we use up one hook in entering it and one in leaving it. There are no odd nodes, therefore the number of hooks at every node is even: hence, if we reach any node except $A$, we shall always find a hook which will take us into a branch previously untraversed. Hence the route will take us finally to the node $A$ from which we started. If there are more than two hooks at $A$, we can continue the route over one of the branches from $A$ previously untraversed, but in the same way as before we shall finally come back to $A$.

It remains to show that we can arrange our route so as to make it cover all the branches. Suppose each branch of the network to be represented by a string with a hook at each end, and that at each node all the hooks there are fastened .. together. The number of hooks at each node is even, and if they are unfastened they can be re-coupled together in pairs, the arrangement of the pairs being immaterial. The whole network will then form one or more closed curves, since now each node consists merely of two ends hooked together.

If this random coupling gives us one single curve then the proposition is proved; for starting at any point we shall go along every branch and come back to the initial point. But 
if this random coupling produces anywhere an isolated loop, $L$, then where it touches some other loop, $M$, say at the node $P$, unfasten the four hooks there (viz. two of the loop $L$ and two of the loop $M$ ) and re-couple them in any other order: then the loop $L$ will become a part of the loop $M$. In this way, by altering the couplings, we can transform gradually all the separate loops into parts of only one loop.

For example, take the case of three isles, $A, B, C$, each connected with both the others by two bridges. The most unfavourable way of re-coupling the ends at $A, B, C$ would be to make $A B A, A C A$, and $B C B$ separate loops. The loops $A B A$ and $A C A$ are separate and touch at $A$; hence we should re-couple the hooks at $A$ so as to combine $A B A$ and $A C A$ into

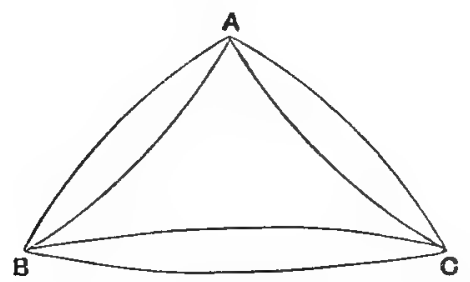

one loop $A B A C A$. Similarly, by re-arranging the couplings of the four hooks at $B$, we can combine the loop $B C B$ with $A B A C A$ and thus make only one loop.

I infer from Euler's language that he had attempted to solve the problem of giving a practical rule which would enable one to describe such a figure unicursally without knowledge of its form, but that in this he was unsuccessful. He however added that any geometrical figure can be described completely in a single route provided each part of it is described twice and only twice, for, if we suppose that every branch is duplicated, there will be no odd nodes and the figure is unicursal. In this case any figure can be described completely without knowing its form: rules to effect this are given below.

Third. A figure which has two and only two odd nodes can be described unicursally by a point which starts from one of the odd nodes and finishes at the other odd node. 
This at once reduces to the second theorem. Let $A$ and $Z$ be the two odd nodes. First, suppose that $Z$ is not a free end. We can, of course, take a route from $A$ to $Z$; if we imagine the branches in this route to be eliminated, it will remove one hook from $\boldsymbol{A}$ and make it even, will remove two hooks from every node intermediate between $A$ and $Z$ and therefore leave each of them even, and will remove one hook from $Z$ and therefore will make it even. All the remaining network is now even: hence, by Euler's second proposition, it can be described unicursally, and, if the route begins at $Z$, it will end at $Z$. Hence, if these two routes are taken in succession, the whole figure will be described unicursally, beginning at $A$ and ending at $Z$. Second, if $Z$ is a free end, then we must travel from $Z$ to some node, $Y$, at which more than two branches meet. Then a route from $A$ to $Y$ which covers the whole figure exclusive of the path from $Y$ to $Z$ can be determined as before and must be finished by travelling from $Y$ to $Z$.

Fourth. A figure having 2 odd nodes, and no more, can be described completely in $n$ separate routes, $n$ being a positive number.

If any route starts at an odd node, and if it is continued until it reaches a node where no fresh path is open to it, this latter node must be an odd one. For every time we enter an even node there is necessarily a way out of it; and similarly every time we go through an odd node we use up one hook in entering and one hook in leaving, but whenever we reach it as the end of our route we use only one hook. If this route is suppressed there will remain a figure with $2 n-2$ odd nodes. Hence $n$ such routes will leave one or more networks with only even nodes. But each of these must have some node common to one of the routes already taken and therefore can be described as a part of that route. Hence the complete passage will require $n$ and not more than $n$ routes. It follows, as stated by Euler, that, if there are more than two odd nodes, the figure cannot be traversed completely in one route. 
The Königsberg bridges lead to a network with four odd nodes; hence, by Euler's fourth proposition, it cannot be described unicursally in a single journey, though it can be traversed completely in two separate routes.

The first and second diagrams figured below contain only even nodes, and therefore each of them can be described unicursally. The first of these is a regular re-entrant pentagon; the second is the so-called sign-manual of Mohammed, said to have been originally traced in the sand by the point of his scimetar without taking it off the ground or retracing any part of the figure-which, as it contains only even nodes, is possible. The third diagram is taken from Tait's article: it contains only two odd nodes, and therefore can be described unicursally if we start from one of them, and finish at the other.
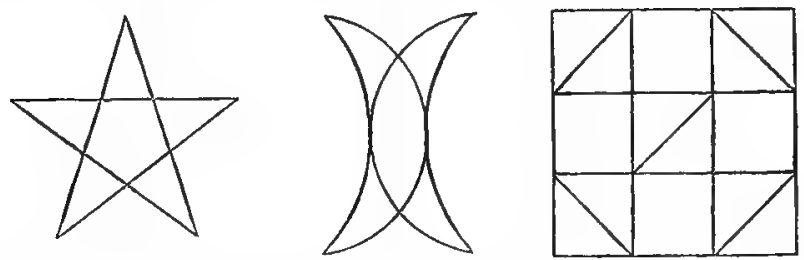

The re-entrant pentagon, figured above, has some interest from having been used by the Pythagoreans as a sign-known as the triple triangle or pentagram star-by which they could recognize one another. It was considered symbolical of health, and probably the angles were denoted by the letters of the word iviec $a$, the diphthong $\epsilon \iota$ being replaced by a $\theta$. Iamblichus, who is our authority for this, tells us that a certain Pythagorean, when travelling, fell ill at a roadside inn where he had put up for the night; he was poor and sick, but the landlord, who was a kind-hearted fellow, nursed him carefully and spared no trouble or expense to relieve his pains. However, in spite of all efforts, the student got worse. Feeling that he was dying and unable to make the landlord any pecuniary recompense, he asked for a board on which he inscribed the pentagram star; this he gave to his host, begging him to hang it up outside so that all passers by might see it, and assuring him that the result 
would recompense him for his charity. The scholar died and was honourably buried, and the board was duly exposed. After a considerable time had elapsed, a traveller one day riding by saw the sacred symbol; dismounting, he entered the inn, and after hearing the story, handsomely remunerated the landlord. Such is the anecdote, which if not true is at least well found.

As another example of a unicursal diagram I may mention the geometrical figure formed by taking a $(2 n+1)$ gon and joining every angular point to every other angular point. The edges of an octahedron also form a unicursal figure. On the other hand a chess-board, divided as usual by straight lines into 64 cells, has 28 odd nodes: hence it would require 14 separate pen-strokes to trace out all the boundaries without going over any more than once. Again, the diagram on page 117 has 20 odd nodes and therefore would require 10 separate pen-strokes to trace it out.

It is well known that a curve which has as many nodes as is consistent with its degree is unicursal.

I turn next to discuss in how many ways we can describe a unicursal figure, all of whose nodes are even *.

Let us consider first how the problem is affected by a path which starts from a node $A$ of order $2 n$ and returns to it, forming a closed loop $L$. If this loop were suppressed we should have a figure with all its nodes even, the node $\boldsymbol{A}$ being now of the order $2(n-1)$. Suppose the original figure can be described in $N$ ways, and the reduced figure in $N^{\prime}$ ways. Then each of these $N^{\prime}$ routes passes $(n-1)$ times through $A$, and in any of these passages we could describe the loop $L$ in either sense as a part of the path. Hence $N=2(n-1) N^{\prime}$.

Similarly if the node $\boldsymbol{A}$ on the original figure is of the order $2(n+l)$, and there are $l$ independent closed loops which start from and return to $A$, we shall have

$$
N=2^{l} n(n+1)(n+2) \ldots(n+l-1) N^{\prime},
$$

where $N^{\prime}$ is the number of routes by which the figure obtained by suppressing these $l$ loops can be described.

* See G. Tarry, Association Française pour l'Avancement des Sciences, 1885, pp. $49-53$.

B. $\mathrm{R}$. 
By the use of these results, we may reduce any unicursal figure to one in which there are no closed loops of the kind above described. Let us suppose that in this reduced figure there are $k$ nodes. We can suppress one of these nodes, say $A$, provided we replace the figure by two or more separate figures each of which has not more than $k-1$ nodes. For suppose that the node $A$ is of the order $2 n$. Then the $2 n$ paths which meet at $A$ may be coupled in $n$ pairs in $1.3 .5 \ldots(2 n-1)$ ways and each pair will constitute either a path through $A$, or (in the special case where both members of the pair abut on another node $B$ ) a loop from $A$. This path or loop will form a portion of the route through $A$ in which this pair of paths are concerned. Hence the number of ways of describing the original figure is equal to the sum of the number of ways of describing 1 . 3.5 ... $(2 n-1)$ separate simpler figures.

It will be seen that the process consists in successively suppressing node after node. Applying this process continually we finally reduce the figure to a number of figures without loops and in each of which there are only two nodes. If in one of these figures these nodes are each of the order $2 n$ it is easily seen that it can be described in $2 \times(2 n-1)$ ! ways.

We know that a figure with only two odd nodes, $A$ and $B$, is unicursal if we start at $A$ (or $B$ ) and finish at $B$ (or $A$ ). Hence the number of ways in which it can be described unicursally will be the same as the number required to describe the figure obtained from it by joining $A$ and $B$. For if we start at $A$ it is obvious that at the $B$ end of each of the routes which cover the figure we can proceed along $B A$ to the node $A$ whence we started.

This theory has been applied by Monsieur Tarry to determine the number of ways in which a set of dominoes, running up to even numbers, can be arranged. This example will serve to illustrate the general method.

A domino consists of a small rectangular slab, twice as long as it is broad, whose face is divided into two squares,

* See the second edition of the French Translation of this work, Paris, 1908, vol. II, pp. 253-263; see also Lucas, vol. Iv, pp. 145-150. 
which are either blank or marked with 1, 2, 3... dots. An ordinary set contains 28 dominoes marked $6-6,6-5,6-4,6-3$, $6-2,6-1,6-0,5-5,5-4,5-3,5-2,5-1,5-0,4-4,4-3,4-2,4-1$, $4-0,3-3,3-2,3-1,3-0,2-2,2-1,2-0,1-1,1-0$, and $0-0$. Dominoes are used in various games in most, if not all, of which the pieces are played so as to make a line such that consecutive squares of adjacent dominoes are marked alike. Thus if $6-3$ is on the table the only dominoes which can be placed next to the 6 end are 6-6, 6-5, 6-4, 6-2, 6-1, or 6-0. Similarly the dominoes $3-5,3-4,3-3,3-2,3-1$, or $3-0$, can be placed next to the 3 end. Assuming that the doubles are played in due course, it is easy to see that such a set of dominoes will form a closed circuit*. We want to determine the number of ways in which such a line or circuit can be formed.

Let us begin by considering the case of a set of 15 dominoes marked up to double-four. Of these 15 pieces, 5 are doubles. The remaining 10 dominoes may be represented by the sides and diagonals of a regular pentagon 01, 02, \&c. The intersections of the diagonals do not enter into the representation,

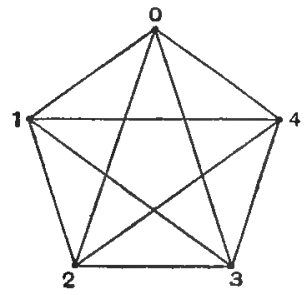

Figure 4 .

and accordingly are to be neglected. Omitting these from our consideration, the figure formed by the sides and diagonals of the pentagon has five even nodes, and therefore is unicursal. Any unicursal route (ex. gr. 0-1, 1-3, 3-0,0-2, 2-3, 3-4, 4-1, $1-2,2-4,4-0$ ) gives one way of arranging these 10 dominoes. Suppose there are $a$ such routes. In any such route we may put each of the five doubles in any one of two positions (ex. gr.

* Hence if we remove one domino, say 5-4, we know that the line formed by the rest of the dominoes must end on one side in $a 5$ and on the other in a 4. 
in the route given above the double-two can be put between 0-2 and 2-3 or between 1-2 and 2-4). Hence the total number of unicursal arrangements of the 15 dominoes is $2^{5} a$. If we arrange the dominoes in a straight line, then as we may begin with any of the 15 dominoes, the total number of arrangements is $15.2^{5} . a$.

We have next to find the number of unicursal routes of the pentagon delineated above in figure $A$. At the node 0 there are four paths which may be coupled in three pairs. If 01 and 02 are coupled, as also 03 and 04 , we get figure $B$. If 01 and 03 are coupled, as also 02 and 04 , we get figure $C$. If 01 and 04 are coupled, as also 02 and 03 , we get figure $D$.

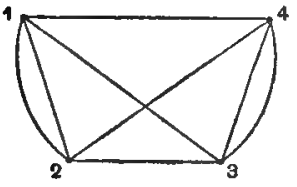

Figure $\boldsymbol{B}$.

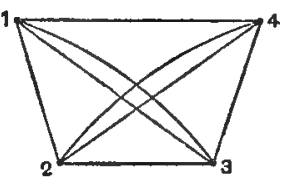

Figure $C$.

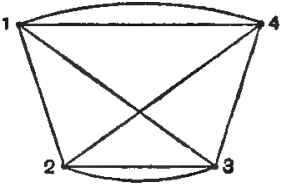

Figure $D$.

Let us denote the number of ways of describing figure $B$ by $b$, of describing figure $C$ by $c$, and so on. The effect of suppressing the node 0 in the pentagon $A$ is to give us three quadrilaterals, $B, C, D$. And, in the above notation, we have $a=b+c+d$.

Take any one of these quadrilaterals, for instance $D$. We can suppress the node 1 in it by coupling the four paths which meet there in pairs. If we couple 12 with the upper of the paths 14 , as also 13 with the lower of the paths 14 , we get

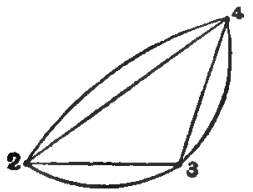

Figure $E$.

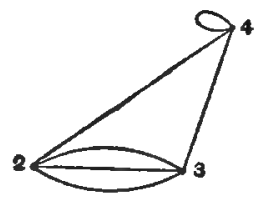

Figure $F$.

the figure $E$. If we couple 12 with the lower of the paths 14 , as also 13 with the upper of the paths 14 , we again get the figure $E$. If we couple 12 and 13 , as also the two paths 14 , we get the figure $F$. Then as above, $d=2 e+f$. Similarly $b=2 e+f$, and $c=2 e+f$. Hence $a=b+c+d=6 e+3 f$. 
We proceed to consider each of the reduced figures $E$ and $F$. First take $E$, and in it let us suppress the node 4 . For simplicity of description, denote the two paths 02 by $\beta$ and $\beta^{\prime}$, and the two paths 43 by $\gamma$ and $\gamma^{\prime}$. Then we can couple $\beta$ and $\gamma$, as also $\beta^{\prime}$ and $\gamma^{\prime}$, or we can couple $\beta$ and $\gamma^{\prime}$, as also $\beta^{\prime}$ and $\gamma$ : each of these couplings gives the figure $G$. Or we can couple $\beta$ and $\beta^{\prime}$, as also $\gamma$ and $\gamma^{\prime}$ : this gives the figure $H$. Thus $e=2 g+h$. Each of the figures $G$ and $H$ has only two nodes. Hence by the formulae given above, we have $g=2.3 \cdot 2=12$, and $h=2 \cdot 2 \cdot 2=8$. Therefore $e=2 g+h=32$. Next take the figure $F$. This has a loop at 4 . If we suppress this

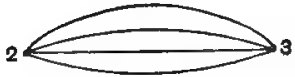

Figure $\boldsymbol{G}$.

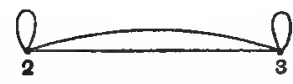

Figure $\boldsymbol{H}$.

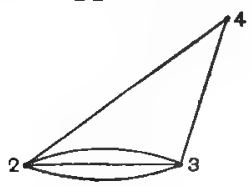

Figure J.

loop we get the figure $J$, and $f=2 j$. But the figure $J$, if we couple the two lines which meet at 4 , is equivalent to the figure $G$. Thus $f=2 j=2 g=24$. Introducing these results we have $a=6 e+3 f=192+72=264$. And therefore $N=15.2^{5}, a=126720$. This gives the number of possible arrangements in line of a set of 15 dominoes. In this solution we have treated an arrangement from right to left as distinct from one which goes from left to right: if these are treated as identical we must divide the result by 2 . The number of arrangements in a closed ring is $2^{5} a$, that is 8448 .

We have seen that this number of unicursal routes for a pentagon and its diagonals is 264. Similarly the number for a heptagon is $h=129976320$. Hence the number of possible arrangements in line of the usual set of 28 dominoes, marked up to double-six, is $28.3^{7}$. $h$, which is equal to 7959229931520 . The number of unicursal routes covering a polygon of nine sides is $n=2^{17} \cdot 3^{11} \cdot 5^{2} \cdot 711.40787$. Hence the number of possible arrangements in line of a set of 45 dominoes marked up to double-eight is $48.4^{9} . n^{*}$.

- These numerical conclusiong have also been obtained by algebraical analysis: see M. Reiss, Annali di Matematica, Milan, 1871, vol, 7, pp. 63-120. 
Mayss. Everyone has read of the labyrinth of Minos in Crete and of Rosamund's Bower. A few modern mazes exist here and there-notably one, a very poor specimen of its kind, at Hampton Court-and in one of these, or at any rate on a drawing of one, most people have at some time threaded their way to the interior. I proceed now to consider the manner in which any such construction may be completely traversed even by one who is ignorant of its plan.

The theory of the description of mazes is included in Euler's theorems given above. The paths in the maze are what previously we have termed branches, and the places where two or more paths meet are nodes. The entrance to the maze, the end of a blind alley, and the centre of the maze are free ends and therefore odd nodes.

If the only odd nodes are the entrance to the maze and the centre of it-which will necessitate the absence of all blind alleys-the maze can be described unicursally. This follows from Euler's third proposition. Again, no matter how many odd nodes there may be in a maze, we can always find a route which will take us from the entrance to the centre without retracing our steps, though such a route will take us through only a part of the maze. But in neither of the cases mentioned in this paragraph can the route be determined without a plan of the maze.

A plan is not necessary, however, if we make use of Euler's suggestion, and suppose that every path in the maze is duplicated. In this case we can give definite rules for the complete description of the whole of any maze, even if we are entirely ignorant of its plan. Of course to walk twice over every path in a labyrinth is not the shortest way of arriving at the centre, but, if it is performed correctly, the whole maze is traversed, the arrival at the centre at some point in the course of the route is certain, and it is impossible to lose one's way.

I need hardly explain why the complete description of such a duplicated maze is possible, for now every node is even, and hence, by Euler's second proposition, if we begin at the entrance we can traverse the whole maze; in so doing we 
shall at some point arrive at the centre, and finally shall emerge at the point from which we started. This description will require us to go over every path in the maze twice, and as a matter of fact the two passages along any path will be always made in opposite directions.

If a maze is traced on paper, the way to the centre is generally obvious, but in an actual labyrinth it is not so easy to find the correct route unless the plan is known. In order to make sure of describing a maze without knowing its plan it is necessary to have some means of marking the paths which we traverse and the direction in which we have traversed them -for example, by drawing an arrow at the entrance and end of every path traversed, or better perhaps by marking the wall on the right-hand side, in which case a path may not be entered when there is a mark on each side of it.

Of the various practical rules for threading a maze those enunciated by M. Trémaux seem to be the simplest*. These I proceed to explain. For brevity I shall describe a path or a node as old or new according as it has been traversed once before or not at all. Then the rules are (i) whenever you come to a new node, take any path you like; (ii) whenever you come by a new path to an old node or to the closed end of a blind alley, turn back along the path by which you have just come; (iii) whenever you come by an old path to an old node, take a new path, if there is one, but if not, an old path; (iv) of course a path traversed twice must not be entered. I should add that on emerging at any node then, of the various routes which are permitted by these rules, it will be convenient always to select that which lies next to one's right hand, or always that which lies next to one's left hand.

Few if any mazes of the type $I$ have been considering (namely, a series of interlacing paths through which some route can be obtained leading to a space or building at the centre of the maze) existed in classical or medieval times. One class of what the ancients called mazes or labyrinths seems to have comprised any complicated building with numerous

- Lucas, vol. 1, part iii, p. 47 et seq. 
vaults and passages". Such a building might be termed a labyrinth, but it is not what is now usually understood by the word. The above rules would enable anyone to traverse the whole of any structure of this kind. I do not know if there are any accounts or descriptions of Rosamund's Bower other than those by Drayton, Bromton, and Knyghton: in the opinion of some, these imply that the bower was merely a house, the passages in which were confusing and ill-arranged.

Another class of ancient mazes consisted of a tortuous path confined to a small area of ground and leading to a tree or shrine in the centref. This is a maze in which there is no chance of taking a wrong turning; but, as the whole area can be occupied by the windings of one path, the distance to be traversed from the entrance to the centre may be considerable, even though the piece of ground covered by the maze is but small.

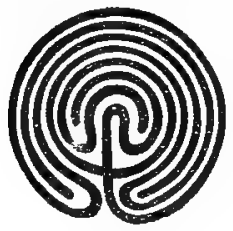

Figure i.

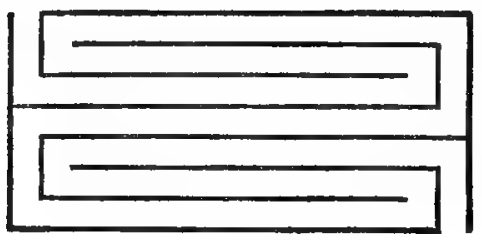

Figure ii.

The traditional form of the labyrinth constructed for the Minotaur is a specimen of this class. It was delineated on the reverses of the coins of Cnossus, specimens of which are not uncommon; one form of it is indicated in the accompanying diagram (figure i). The design really is the same as that

- For instance, see the descriptions of the labyrinth at Lake Moeris given by Herodotus, bk. ii, c. 148; Strabo, bk. xvii, u. 1, art. 37; Diodorus, bk. i, cc. 61,66 ; and Pliny, Hist. Nat., bk. xxzvi, c. 13, arts. 84-89. On these and other references see A. Wiedemann, Herodots zweites Buch, Leipzig, 1890, p. 522 et seq. See also Virgil, Aeneid, bk. v, 0. v, 588; Ovid, Met., bk. viii, c. 5, 159; Strabo, bk. viii, o. 6.

+ On ancient and medieval labyrintbs-particularly of this kind-see an article by Mr E. Trollope in The Archaeological Journal, 1858, vol. Xv, pp. 216235 , from which muoh of the historical information given above is derived. 
drawn in figure ii, as can be easily seen by bending round a circle the rectangular figure there given.

Mr Inwards has suggested* that this design on the coins of Cnossus may be a survival from that on a token given by the priests as a clue to the right path in the labyrinth there. Taking the circular form of the design shown above he supposed each circular wall to be replaced by two equidistant walls separated by a path, and thus obtained a maze to which the original design would serve as the key. The route thus indicated may be at once obtained by noticing that when a node is reached (i.e. a point where there is a choice of paths) the path to be taken is that which is next but one to that by which the node was approached. This maze may be also threaded by the simple rule of always following the wall on the right-hand side or always that on the left-hand side. The labyrinth may be somewhat improved by erecting a few additional barriers, without affecting the applicability of the above rules, but it cannot be made really difficult. This makes a pretty toy, but though the conjecture on which it is founded is ingenious it has no historical justification. Another suggestion is that the curved line on the reverse of the coins indicated the form of the rope held by those taking part in some rhythmic dance; while others consider that the form was gradually evolved from the widely prevalent svastika.

Copies of the maze of Cnossus were frequently engraved on Greek and Roman gems; similar but more elaborate designs are found in numerous Roman mosaic pavements $\dagger_{\text {. A copy }}$ of the Cretan labyrinth was embroidered on many of the state robes of the later Emperors, and, apparently thence, was copied on to the walls and floors of various churchest. At a later time in Italy and in France these mural and pavement decorations were developed into scrolls of great complexity, but consisting, as far as I know, always of a single line. Some of the best specimens now extant are on the walls of the

* Knowledge, London, October, 1892.

† See ex. gr. Breton's Pompeia, p. 303.

$\ddagger$ Ozanam, Graphia aureae urbis Romae, pp. 92, 178 . 
cathedrals at Lucca, Aix in Provence, and Poitiers; and on the floors of the churches of Santa Maria in Trastevere at Rome, San Vitale at Ravenna, Notre Dame at St Omer, and the cathedral at Chartres. It is possible that they were used to represent the journey through life as a kind of pilgrim's progress.

In England these mazes were usually, perhaps always, cut in the turf adjacent to some religious house or hermitage: and there are some slight reasons for thinking that, when traversed as a religious exercise, a pater or ave had to be repeated at every turning. After the Renaissance, such labyrinths were frequently termed Troy-Towns or Julian's Bowers. Some of the best specimens, which are still extant, or were so until recently, are those at Rockliff Marshes, Cumberland; Asenby, Yorkshire; Alkborough, Lincolnshire; Wing, Rutlandshire; Boughton-Green, Northamptonshire; Comberton, Cambridgeshire; Saffron Walden, Essex; and Chilcombe, near Winchester.

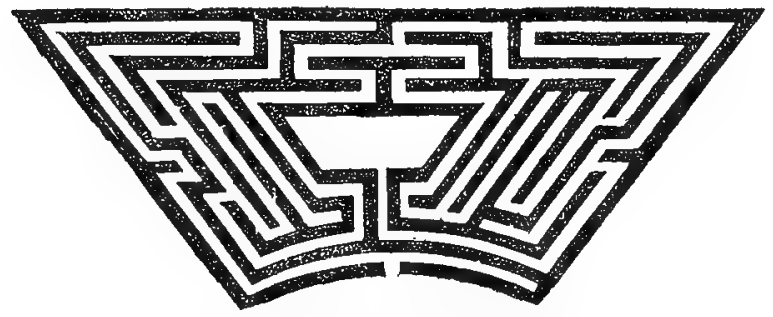

Mate at Hampton Court.

The modern maze seems to have been introduced-probably from Italy-during the Renaissance, and many of the palaces and large houses built in England during the Tudor and the Stuart periods had labyrintbs attached to them. Those adjoining the royal palaces at Southwark, Greenwich, and Hampton Court were well known from their vicinity to the capital. The last of these was designed by London and Wise in 1690, for William III, who had a fancy for such conceits: a plan of it is given in various guide-books. For the majority of the sight-seers who enter, it is sufficiently 
elaborate; but it is an indifferent construction, for it can be described completely by always following the hedge on one side (either the right hand or the left hand), and no node is of an order higher than three.

Unless at some point the route to the centre forks and subsequently the two forks reunite, forming a loop in which the centre of the maze is situated, the centre can be reached by the rule just given, namely, by following the wall on one side-either on the right hand or on the left hand. No labyrinth is worthy of the name of a puzzle which can be threaded in this way. Assuming that the path forks as described above, the more numerous the nodes and the higher their order the more difficult will be the maze, and the difficulty might be increased considerably by using bridges and tunnels so as to construct a labyrinth in three dimensions. In an ordinary garden and on a small piece of ground, often of an inconvenient shape, it is not easy to make a maze which fulfils these conditions. Here is a plan of one which I put up

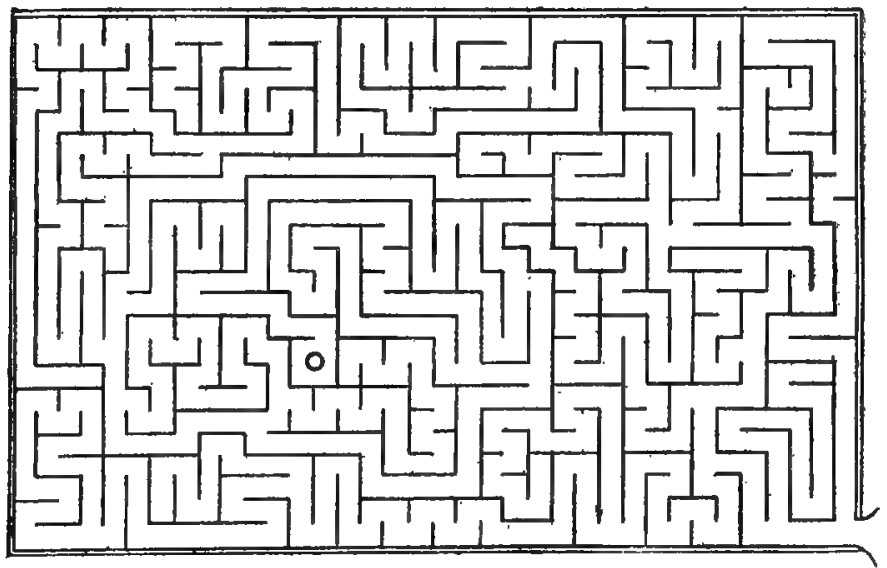

in my own garden on a plot of ground which would not allow of more than 36 by 23 paths, but it will be noticed that none of the nodes are of a high order. 
Geometrical Trees. Euler's original investigations were confined to a closed network. In the problem of the maze it was assumed that there might be any number of blind alleys in it, the ends of which formed free nodes. We may now progress one step further, and suppose that the network or closed part of the figure diminishes to a point. This last arrangement is known as a tree. The number of unicursal descriptions necessary to completely describe a tree is called the base of the ramification.

We can illustrate the possible form of these trees by rods, having a hook at each end. Starting with one such rod, we can attach at either end one or more similar rods. Again, on any free hook we can attach one or more similar rods, and so on. Every free hook, and also every point where two or more rods meet, are what hitherto we have called nodes. The rods are what hitherto we have termed branches or paths.

The theory of trees-which already plays a somewhat important part in certain branches of modern analysis, and possibly may contain the key to certain chemical and biological theories-originated in a mernoir by Cayley*, written in 1856. The discussion of the theory has been analytical rather than geometrical. I content myself with noting the following results.

The number of trees with $n$ given nodes is $n^{n-2}$. If $A_{n}$ is the number of trees with $n$ branches, and $B_{n}$ the number of trees with $n$ free branches which are bifurcations at least, then

$$
\begin{aligned}
& (1-x)^{-1}\left(1-x^{2}\right)^{-A_{1}}\left(1-x^{3}\right)^{-A_{3}} \ldots \ldots=1+A_{1} x+A_{2} x^{2}+A_{3} x^{3}+\ldots, \\
& (1-x)^{-1}\left(1-x^{3}\right)^{-B_{2}}\left(1-x^{3}\right)^{-B_{3}} \ldots \ldots=1+x+2 B_{2} x^{2}+2 B_{3} x^{3}+\ldots .
\end{aligned}
$$

- Philosophical Magazine, March, 1857, series 4, vol. xmr, pp. 172-176; or Collected Works, Cambridge, 1890, vol. III, no. 203, pp. 242-216: see also the paper on double partitions, Philosophical Magazine, November, 1860, series 4, vol. xx, pp. 337-341. On the number of trees with a given number of nodes, see the Quarterly Journal of Mathematics, London, 1889, vol. xxIII, pp. 376-378. The connection with chemistry was first pointed out in Cayley's paper on isomers, Philosophical Magazine, June, 1874, series 4, vol. xLvI, pp. 444-447, and was treated more fully in his report on trees to the British Association in 1875, Reports, pp. 257-305. 
Using these formulae we can find successively the values of $A_{1}, A_{2}, \ldots$, and $B_{1}, B_{3}, \ldots$. The values of $A_{n}$ when $n=2,3,4$, $5,6,7$, are $2,4,9,20,48,115$; and of $B_{n}$ are $1,2,5,12,33$, 90

I turn next to consider some problems where it is desired to find a route which will pass once and only once through each node of a given geometrical figure. This is the reciprocal of the problem treated in the first part of this chapter, and is a far more difficult question. I am not aware that the general theory has been considered by mathematicians, though two special cases-namely, the Hamiltonian (or Icosian) Game and the Knight's Path on a Chess-Board-have been treated in some detail.

The Hamiltonian Game. The Hamiltonian Game consists in the determination of a route along the edges of a regular dodecahedron which will pass once and only once through every angular point. Sir William Hamilton*, who invented this game-if game is the right term for it-denoted the twenty angular points on the solid by letters which stand for various towns. The thirty edges constitute the only possible paths. The inconvenience of using a solid is considerable, and the dodecahedron may be represented conveniently in
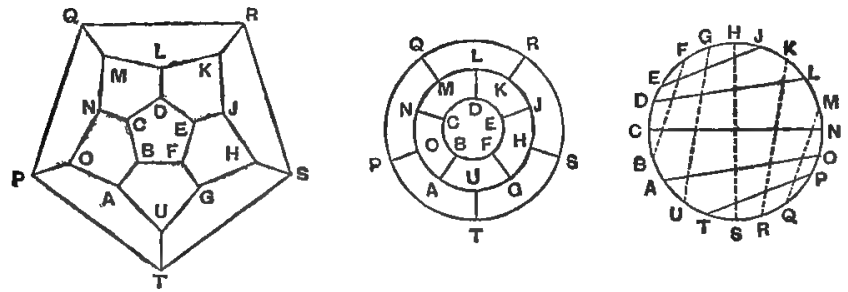

perspective by a flat board marked as shown in the first of the annexed diagrams. The second and third diagrams will answer our purpose equally well and are easier to draw.

* See Quarterly Journal of Mathematics, London, 1862, vol. v, p. 305; or Philosophical Magazine, January, 1884, serios 5, vol. zriI, p. 42; also Lucas, vol. Ir, part vii. 
The first problem is to go "all round the world," that is, starting from any town, to go to every other town once and only once and to return to the initial town; the order of the $n$ towns to be first visited being assigned, where $n$ is not greater than five.

Hamilton's rule for effecting this was given at the meeting in 1857 of the British Association at Dublin. At each angular point there are three and only three edges. Hence, if we approach a point by one edge, the only routes open to us are one to the right, denoted by $r$, and one to the left, denoted by $l$. It will be found that the operations indicated on opposite sides of the following equalities are equivalent,

$$
l r^{2} l=r l r, r l^{2} r=l r l, l r^{3} l=r^{2}, r l^{8} r=l^{2} .
$$

Also the operation $l^{5}$ or $r^{5}$ brings us back to the initial point: we may represent this by the equations

$$
l^{5}=1, r^{5}=1 \text {. }
$$

To solve the problem for a figure having twenty angular points we must deduce a relation involving twenty successive operations, the total effect of which is equal to unity. By repeated use of the relation $l^{2}=r l^{3} r$ we see that

$$
\begin{aligned}
1=l^{5}=l^{2} l^{3}=\left(r l^{3} r\right) l^{8} & =\left\{r l^{8}\right\}^{2}=\left\{r\left(r l^{3} r\right) l\right\}^{3} \\
& =\left\{r^{2} l^{8} r l\right\}^{2}=\left\{r^{2}\left(r l^{3} r\right) l r l\right\}^{2}=\left\{r^{3} l^{3} r l r l\right\}^{2} .
\end{aligned}
$$

Therefore

$$
\left\{r^{3} l^{3}(r l)^{2}\right\}^{2}=1
$$

and similarly

$$
\left\{l^{s} r^{3}(l r)^{2}\right\}^{2}=1
$$

Hence on a dodecahedron either of the operations

$$
\begin{aligned}
& r r r l l l r l r l r r r l l l r l r l \ldots \text { (i), } \\
& l l l r r r l r l r l l l r r r l r l r \ldots \text { (ii), }
\end{aligned}
$$

indicates a route which takes the traveller through every town. The arrangement is cyclical, and the route can be commenced at any point in the series of operations by transferring the proper number of letters from one end to the other. The point at which we begin is determined by the order of certain towns which is given initially. 
Thus, suppose that we are told that we start from $\not F$ and then successively go to $B, A, U$, and ' $T$, and we want to find a route from $T$ through all the remaining towns which will end at $F$. If we think of ourselves as coming into $F$ from $G$, the path $F B$ would be indicated by $l$, but if we think of ourselves as coming into $F$ from $E$, the path $F B$ would be indicated by $r$. The path from $B$ to $A$ is indicated by $l$, and so on. Hence our first paths are indicated either by $l l l r$ or by $r l l r$. The latter operation does not occur either in (i) or in (ii), and therefore does not fall within our solutions. The former operation may be regarded either as the 1st, 2nd, 3rd, and 4th steps of (ii), or as the 4th, 5th, 6th, and 7th steps of (i). Each of these leads to a route which satisfies the problem. These routes are

\section{FBA UTPONCDEJKLMQRSHG $F$,}

\section{and $F B A T T S R K L M Q P O N C D E J H G F$.}

It is convenient to make a mark or to put down a counter at each corner as soon as it is reached, and this will prevent our passing through the same town twice.

A similar game may be played with other solids provided that at each angular point three and only three edges meet. Of such solids a tetrahedron and a cube are the simplest instances, but the reader can make for himself any number of plane figures representing such solids similar to those drawn on page 189. Some of these were indicated by Hamilton. In all such cases we must obtain from the formulae analogous to those given above cyclical relations like (i) or (ii) there given. The solution will then follow the lines indicated above. This method may be used to form a rule for describing any maze in which no node is of an order higher than three.

For solids having angular points where more than three edges meet-such as the octahedron where at each angular point four edges meet, or the icosahedron where at each angular point five edges meet-we should at each point have more than two routes open to us; hence (unless we suppress some of the edges) the symbolical notation would have to be 
extended before it could be applied to these solids. I offer the suggestion to anyone who is desirous of inventing a new game.

Another and a very elegant solution of the Hamiltonian dodecahedron problem has been given by $\mathbf{M}$. Hermary. It consists in unfolding the dodecahedron into its twelve pentagons, each of which is attached to the preceding one by only one of its sides; but the solution is geometrical, and not directly applicable to more complicated solids.

Hamilton suggested as another problem to start from any town, to go to certain specified towns in an assigned order, then to go to every other town once and only once, and to end the journey at some given town. He also suggested the consideration of the way in which a certain number of towns should be blocked so that there was no passage through them, in order to produce certain effects. These problems have not, so far as I know, been subjected to mathematical analysis.

The problem of the knight's path on a chess-board is somewhat similar in character to the Hamiltonian game. This I have already discussed in chapter $\nabla \mathbf{~}$. 


\section{CHAPTER X.}

\section{KIRKMAN'S SCHOOL-GIRLS PROBLEM.}

The Fifteen School-Girls Problem-first enunciated by T. P. Kirkman, and commonly known as Kirkman's Problem - consists in arranging fifteen things in different sets of triplets. It is usually presented in the form that a schoolmistress was in the habit of taking her girls for a daily walk. The girls were fifteen in number, and were arranged in five rows of three each so that each girl might have two companions. The problem is to dispose them so that for seven consecutive days no girl will walk with any of her school-fellows in any triplet more than once.

In the general problem, here discussed, we require to arrange $n$ girls, where $n$ is an odd multiple of 3 , in triplets to walk out for $y$ days, where $y=(n-1) / 2$, so that no girl will walk with any of her school-fellows in any triplet more than once.

The theory of the formation of all such possible triplets in the case of nine girls is comparatively easy, but the general theory involves considerable difficulties. Before describing any methods of solution, I will give briefly the leading facts in the history of the problem. For this and much of the material of this chapter I am indebted to O. Eckenstein. Detailed references to the authorities mentioned are given in the bibliography mentioned in the footnote*.

* The problem was first published in the Lady's and Gentleman's Diary for 1850, p. 48 , and has been the subject of numerous memoirs. A bibliography of the problem by 0 . Eckenstein appeared in the Messenger of Mathematics, Cambridge, July, 1911, vol. zul, pp. 33-36.

B. $\mathbf{R}$. 
The question was propounded in 1850 , and in the same year solutions were given for the cases when $n=9,15$, and 27 ; but the methods used were largely empirical.

The first writer to subject it to mathematical analysis was R. R. Anstice who, in 1852 and 1853, described a method for solving all cases of the form $12 m+3$ when $6 m+1$ is prime. He gave solutions for the cases when $n=15,27,39$. Substantially, his process, in a somewhat simplified form, is covered by that given below under the heading Analytical Methods.

The next important advance in the theory was due to B. Peirce who, in 1860, gave cyclical methods for solving all cases of the form $12 m+3$ and $24 m+9$. But the processes used were complicated and partly empirical.

In 1871 A. H. Frost published a simple method applicable to the original problem when $n=15$ and to all cases when $n$ is of the form $2^{2 m}-1$. It has been applied to find solutions when $n=15$ and $n=63$.

In 1883 A. Bray (a name assumed by G. D. L. Harison) and E. Marsden gave three-step cyclical solutions for 21 girls. These were interesting because Kirkman had expressed the opinion that this case was insoluble.

Another solution when $n=21$, by T. $H$. Gill, was given in the fourth edition of this book in 1905. His method though empirical appears to be applicable to all cases, but for high values of $n$ it involves so much preliminary work by trial and error as to be of little value.

A question on the subject which I propounded in the Educational Times in 1906, attracted the attention of L. A. Legros, H. E. Dudeney and O. Eckenstein, and I received from them a series of interesting and novel solutions. As illustrations of the processes used, Dudeney published new solutions for $n=27,33,51,57,69,75,87,93,111$; and Eckenstein for $n=27,33,39,45,51,57,69,75,93,99,111,123,135$.

I now proceed to describe some of the methods applicable to the problem. We can use cycles and combinations of them. I confine my discussion to processes where the steps of the cycles do not exceed three symbols at a time. It will be convenient to begin with the easier methods, where however a 
certain amount of arrangement has to be made empirically, and then to go on to the consideration of the more general method.

One-Step Cycles. As illustrating solutions by one-step cyclical permutations I will first describe Legros's method. Solutions obtained by it can be represented by diagrams, and their use facilitates the necessary arrangements. It is always applicable when $n$ is of the form $24 m+3$, and seems to be also applicable when $n$ is of the form $24 m+9$. Somewhat similar methods were used by Dudeney, save that he made no use of geometrical constructions.

We have $n=2 y+1=24 m+3$ or $n=2 y+1=24 m+9$. We may denote one girl by $k$, and the others by the numbers $1,2,3, \ldots 2 y$. Place $k$ at the centre of a circle, and the numbers $1,2,3, \ldots 2 y$ at equidistant intervals on the circumference. Thus the centre of the circle and each point on its circumference will indicate a particular girl. A solution in which the centre of the circle is used to denote one girl is termed a central solution.

The companions of $k$ are to be different on each day. If we suppose that on the first day they are 1 and $y+1$, on the second 2 and $y+2$, and so on, then the diameters through $k$ will give for each day a triplet in which $k$ appears. On each day we have to find $2(y-1) / 3$ other triplets satisfying the conditions of the problem. Every triplet formed from the remaining $2 y-2$ girls will be represented by an inscribed triangle joining the points representing these girls. The sides of the triangles are the chords joining these $2 y-2$ points. These chords may be represented symbolically by [1], [2], $[3], \ldots[y-1]$; these numbers being proportional to the smaller arcs subtended. I will denote the sides of a triangle so represented by the letters $p, q, r$, and I will use the term triad or grouping to denote any group of $p, g, r$ which determines the dimensions of an inscribed triangle. I shall place the numbers of a triad in square brackets. If $p, q, r$ are proportional to the smaller arcs subtended, it is clear that if $p+q$ is less than $y$, we have $p+q=r$; and if $p+q$ is greater than $y$ we have $p+q+r=2 y$. If we like to use arcs larger than the 
semi-circumference we may confine ourselves to the relation $p+q=r$. In the geometrical methods described below, we usually first determine the dimensions of the triangles to be used in the solution, and then find how they are to be arranged in the circle.

If $(y-1) / 3$ scalene triangles, whose sides are $p, q, r$, can be inscribed in the circle so that to each triangle corresponds an equal complementary triangle having its equal sides parallel to those of the first and with its vertices at free points, then the system of $2(y-1) / 3$ triangles with the corresponding diameter will give an arrangement for one day. If the system be permuted cyclically $y-1$ times we get arrangements for the other y-1 days. No two girls will walk together twice, for each chord occupies a different position after each permutation, and as all the chords forming the $(y-1) / 3$ triangles are unequal the same combination cannot occur twice. Since the triangles are placed in complementary pairs, one being $y$ points in front of the other, it follows that after $y-1$ permutations we shall come to a position like the initial one, and the cycle will be completed. If the circle be drawn and the triangles cut out to scale, the arrangement of the triangles is facilitated. The method will be better understood if $I$ apply it to one or two of the simpler cases.

The first case is that of three girls, $a, b, c$, walking out for one day, that is, $n=3, m=0, y=1$. This involves no discussion, the solution being ( $a . b . c)$.

The next case is that of nine girls walking out for four days, that is, $n=9, m=0, y=4$. The first triplet on the first day is (1.k.5). There are six other girls represented by the points $2,3,4,6,7,8$. These points can be joined so as to form triangles, and each triangle will represent a triplet. We want to fiud one such triangle, with unequal sides, with its vertices at three of these points, and such that the triangle formed by the other three points will have its sides equal and parallel to the sides of the first triangle.

The sides of a triangle are $p, q, r$. The only possible values are $1,2,3$, and they satisfy the condition $p+q=r$. If a 
triangle of this shape is placed with its vertices at the points $3,4,6$, we can construct a complementary equal triangle, four points further on, having $7,8,2$ for its vertices. All the points in the figure are now joined, and form the three triplets for the first day, namely (k. 1.5), (3. 4. 6), (7. 8. 2). It is only necessary to rotate the figure one step at a time in order to obtain the triplets for the remaining three days. Another similar solution is obtained from the diameter (1.k.5), and the triangles (2.3.8), (6. 7. 4). It is the reflection of the former solution.

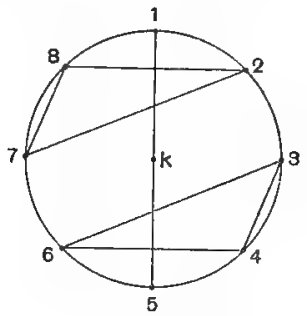

Figure i.

The next case to which the method is applicable is when $n=27, n=1, y=13$. Proceeding as before, the 27 girls must be arranged with one of them, $k$, at the centre and the other 26 on the circumference of a circle. The diameter (1. $k .14$ ) gives the first triplet on the first day. To obtain the other triplets we have to find four dissimilar triangles which satisfy the conditions mentioned above. The chords used as sides of these triangles may be of the lengths represented symbolically by [1], [2], ... [12]. We have to group these lengths so that $p+q=r$ or $p+q+r=2 y$; if the first condition can be satisfied it is the easier to use, as the numbers are smaller. In this instance the triads $[3,8,11],[5,7,12],[2,4,6],[1,9,10]$ will be readily found. Now if four triangles with their sides of these lengths can be arranged in a system so that all the vertices fall on the ends of different diameters (exclusive of the ends of the diameter $1, k, 14$ ), it follows that the opposite ends of those diameters can be joined by chords giving a series of equal triangles, symmetrically placed, each having its sides parallel to those of a triangle of the first system. The following arrangement 
of triangles satisfies the conditions: (4. 11. 25), (5. 8. 23), (6. 7. 16), (9. 13. 15). The complementary system is (17.24.12), (18. 21. 10), (19. 20.3), (22. 26. 2). These triplets with (k. 1. 14) give an arrangement for the first day; and, by rotating the system cyclically, the arrangements for the remaining 12 days can be found immediately.

I proceed to give one solution of this type for every remaining case where $n$ is less than 100. From the result the triads or groupings used can be obtained. It is sufficient in each case to give an arrangement on the first day, since the arrangements on the following days are at once obtainable by oyclical permutations.

I take first the three cases, $33,57,81$, where $n$ is of the form $24 m+9$. In these cases the arrangements on the other days are obtained by one-step oyclical permutations.

For 33 girls, a solution is given by the system of triplets $(2.11 .16)$, (4. 6. 10), (5.13.30), (7. 8. 19), (9.28. 31), and the complementary gystem (18. 27. 32), (20. 22. 26), (21. 29. 14), (23. 24. 3), (25. 12. 15). These 10 triplets, together with that represented by $(k .1 .17)$, will give an arrangement for the first day.

For 57 girls, a possible arrangement of triplets is (18. 13. 50), (20. 11. 28), (21. 52. 3), (8.10. 51), (4. 25. 26), (2.6.12), (7. 19. 33), (27. 43. 16), (37.14. 17). These, with the 9 complementary triplets, and the diameter triplat (1. k. 29), give an arrangement for the first day.

For 81 girls an arrangement for the first day consists of the diameter triplet (1. k. 41), the 13 triplets $(3.35 .42),(4.10 .29),(5.28 .56),(6.26 .39),(7.15 .17)$, (8. 11. 32), (13. 27 49), (14. 19. 30), (20. 37. 38), (21. 25. 52), (24. 36. 62), (18. 33. 63), (31. 40. 74), and the 13 complementary triplets.

I take next the three cases, $51,75,99$, where $n$ is of the form $24 m+3$. In these cases the arrangements on the other days are obtained either by one-step or by two-step cyclical permutations.

For 51 girls, an arrangement for the first day consists of the diameter triplet (k. 1. 26), the 8 triplets (2. 9. 36), (4. 7. 25), (6. 10. 19), (8.14.22), (12.17. 45), (13. 24.48), (15. 16.46), (18.28.30), and the 8 complementary triplets.

For 75 girls, an arrangement for the first day consists of the diameter triplet (k. 1. 38), the 12 triplets (2. 44. 55), (4. 11. 19), (5. 50. 66), (6. 52. 57), (8. 46. 58), (10.59.65), (12.60.64), (14. 24. 68), (16. 25. 72), (17. 3. 74), (33. 34. 73), (30.32.63), and the 12 complementary triplets.

For 99 girls an arrangement for the first day consists of the diameter triplet (1. k. 50), the 16 triplets (2.17. 47), (3.9.68), (4. 44. 82), (5. 12. 75), (6. 32. 42), (7. 23. 97), (8. 21. 30), (15. 20. 76), (16. 35. 85), (18. 45. 62), (22. 40. 63), (25. 37. 92), (28. 29. 80), (34. 38. 59), (39. 41. 73), (46. 49. 60), and the 16 complementary triplets.

It is also possible to obtain, for numbers of the form $24 m+3$, solutions which are uniquely two-step, but in these the complementary triangles are not 
placed aymmetrically to each other. I give 27 girls as an instance, using the same triads as in the solution of this case given above. The triplets for the first day are (k. 1. 14), (2. 12. 3), (21. 5. 22), (20. 24. 26), (11. 15. 17), (8. 16. 19), (25. 7. 10), (6. 18. 13), and (23. 9. 4). From this the arrangements on the other days can be obtained by a two-step (but not by a one-step) oyelical permutation.

It is unnecessary to give more examples, or to enter on the question of how from one solution others can be deduced, or how many solutions of each case can be obtained in this way. The types of the possible triangles are found analytically, but their geometrical arrangement is empirical. The defect of this method is that it may not be possible to arrange a given grouping. Thus when $n=27$, we easily obtain 24 different groupings, but two of them cannot be arranged geometrically to give solutions; and whether any particular grouping will give a solution can, in many cases, be determined only by long and troublesome empirical work. The same objection applies to the two-step and three-step methods which are described below.

Two-Step Cycles. The method used by Legros was extended by Eckenstein to cases where $n$ is of the form $12 m+3$. When $n$ is of this form and $m$ is odd we cannot get sets of complementary triangles as is required in Legros's method; hence, to apply a similar method, we have to find $2(y-1) / 3$ different dissimilar inscribed triangles having no vertex in common and satisfying the condition $p+q=r$ or $p+q+r=2 y$. These solutions are also central. Since there are $2 y$ points on the circumference of the circle the permutations, if they are to be cyclical, must go in steps of two numbers at a time. In Legros's method we represented one triplet by a diameter. But obviously it will answer our purpose equally well to represent it by a triangle with $k$ as vertex and two radii as sides, one drawn to an even number and the other to an odd number: in fact this will include the diameter as a particular case.

I begin by considering the case where we use the diameter (1. k. y) to represent one triplet on the first day. Here the chords used for sides of the triangles representing the other triplets must be of lengths $[1],[2], \ldots[y-1]$. Also each given 
length must appear twice, and the two equal lines so represented must start one from an even number and the other from an odd number, so as to avoid the same combination of points occurring again when the system is rotated cyclically. Of course a vertex cannot be at the point 1 or $y$, as these points will be required for the diameter triplet (1.k. $y$ ).

These remarks will be clearer if we apply them to a definite example. I take as an instance the case of 15 girls. As before we represent 14 of them by equidistant points numbered 1, 2, $3, \ldots 14$ on the circumference of a circle, and one by a point $k$ at its centre. Take as one triplet the diameter (1.k.8). Then the sides $p, q, r$ may have any of the values [1], [2], [3], [4], [5], [6], and each value must be used twice. On examination it will be found that there are only two possible groupings, namely $[1,1,2],[2,4,6],[3,3,6],[5,5,4]$, and $[1,2,3],[1,4,5]$, $[3,5,6],[2,4,6]$. One of the solutions to which the first set of groupings leads is defined by the diameter (1. k.8) and the four triplets (9.10.11), (4. 6. 14), (2.5.13), (3.7.12); see figure ii, below: of the four triangles used three are isosceles. The

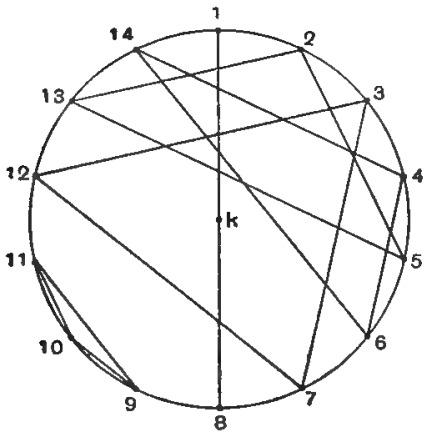

Figure ii.

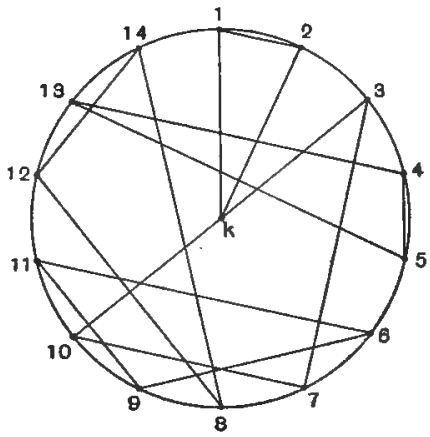

Figure iii.

second set leads to solutions defined by the triplets (k. 1. 8), (4. 5.7), (13.14.9), (3.6.11), (10.12.2), or by the triplets (k.1. 8), (6.7.9), (3. 4.13), (5.11.14), (10.12.2): in these solutions all the triangles used are scalene. If any one of these three sets of triplets is rotated cyclically two steps at 
a time, we get a solution of the problem for the seven days required. Each of these solutions by reflection and inversion gives rise to three others.

Next, if we take (k. 1. 2) for one triplet on the first day we shall have the points $3,4, \ldots 14$ for the vertices of the four triangles denoting the other triplets on that day. The sides must be of the lengths [1], [2],... [7], of which [2],... [6] must be used not more than twice and [1], [7] must be used only once. The [1] used must start from an even number, for otherwise the chord denoted by it would, when the system was rotated, occupy the position joining the points $\mathbf{1}$ and $\mathbf{2}$, which has been already used. The only possible groupings are $[2,4,6],[2,3,5],[3,4,7],[1,5,6]$; or $[2,4,6],[2,5,7],[1,4,5]$, $[3,3,6]$; or $[2,4,6],[2,5,7],[1,3,4],[3,5,6]$. Each of these groupings gives rise to various solutions. For instance the first grouping gives a set of triplets (k. 1. 2), (3. 7. 10), (4. 5. 13), (6. 9.11), (8.12.14). From this by a cyclical two-step permutation we get a solution. This solution is represented in figure iii. If we take (k. 1.4) or $(k .1 .6)$ as one triplet on the first day, we get other sets of solutions.

Solutions involving the triplets $(k .1 .2),(k .1 .4),(k .1 .6)$, (lc. 1. 8), and other analogous solutions, can be obtained from the solutions illustrated in the above diagrams by re-arranging the symbols denoting the girls. For instance, if in figure iii, where all the triangles used are scalene, we replace the numbers $2,8,3,13,4,6,5,11,7,9,10,14$ by $8,2,13,3,6,4,11,5,9$, $7,14,10$, we get the solution (k.1.8), (4.5.7), etc., given above. Again, if in figure iii we replace the symbols $1,2,3,4,5,6$, $7,8,9,10,11,12,13,14, k$ by $12,2,10,6,1,14,5,13,9$, $15,4,3,11,8,7$, we obtain a solution equivalent to that given by T. H. Gill and printed in the fourth edition of this book. In this the arrangement on the first day is (1. 6. 11), (2. 7. 12), (3. 8. 13), (4.9.14), (5.10.15). The arrangements on the other days are obtained as before by rotating the system so delineated round 7 as a centre two steps at a time. Gill's arrangement is thus presented in its canonical form as a central two-step cyclical solution. 
I proceed to give one solution of this type for every remaining case where $n$ is less than 100. In each case I give an arrangement on the first day; the arrangements for the other days can be got from it by a two-step cyclical permutation of the numbers.

In the ease of 27 girls, one arrangement on the first day is (k. 1. 14), (19. 21. 20), (3. 9. 6), (13. 23. 18), (5. 17. 24), (7. 25. 16), (11. 15. 26), (10.4. 2), (12. 22. 8).

In the case of 39 girls, one arrangement for the first day is $(k, 1.20)$, (35. 37. 36), (7. 13. 10), (19. 29. 24), (11. 25. 18), (3. 21. 12), (17. 33. 6), (15. 27. 2), (23. 31. 8), (5. 9. 26), (4. 16.32), (14.34.38), (22. 28. 30).

In the case of 51 girls, one arrangement for the first day is (k. 1. 26), (21. 23. 22), (11. 17. 14), (35. 45. 40), (25. 39. 32), (15. 33. 24), (13. 41. 2), (5. 31. 18), (19. 49. 34), (27. 43. 10), (9. 47. 28), (29. 37. 8), (3. 7. 30), (4. 20.46), (6. 36. 42), (12.16.44), (38. 48. 50).

In the case of 63 girls, one arrangement for the first day is (k. 1. 32), (57. 59. 58), (23. 29. 26), (37. 47. 42), (5. 53. 60), (17.61. 8), (3. 43. 54), (7. 33. 20), (9. 39. 24), (27. 55. 10), (21. 45. 2), (11. 31. 52), (35. 51. 12), (13. 25. 50), (41. 49. 14), (15. 19. 48), (16. 18. 36), (6. 34. 38), (46. 56. 62), (22. 30. 44), (4. 28. 40).

In the case of 75 girls, one arrangement for the first day is (k. 1. 38), (23. 25. 24), (3. 9. 6), (29. 39. 34), (7. 21. 14), (51. 69.60), (33. 55. 44), (11. 59. 72), (35. 65. 50), (37. 71. 54), (27. 63. 8), (15.57. 36), (13. 41. 64), (43. 67. 18), (19. 73. 46), (31. 47. 2), (5. 17. 48), (53. 61. 20), (45. 49. 10), (30. 32. 52), (22. 26.66), (56.62.70), (12.28. 74), (16. 40.58), (4. 42.68).

In the case of 87 girls, one arrangement for the first day is (k. 1. 44), (61. 63. 62), (73. 79. 76), (35. 45. 40), (11. 83. 4), (25. 43. 34), (59. 81. 70), (7. 33. 20), (41. 71. 56), (23. 75.6), (27.65. 46), (13. 57. 78), (5. 51. 28), (3. 39. 64), (15. 47. 74), (9. 67. 38), (31. 55. 86), (19. 85. 52), (21. 37. 72), (17. 29. 66), (69. 77. 30), (49. 53. 8), (10. 12. 24), (22. 26. 84), (18. 54. 60), (2. 50. 80), (32. 42. 58), (14. 36. 82), (16. 48. 68).

In the case of 99 girls, one arrangement for the first day is (k. 1. 50), (47. 49. 48), (53. 59. 56), (55.65.60), (57.71.64), (23. 41. 32), (17. 39. 28), (63. 89. 76), (5. 35. 20), (3. 67. 84), (9. 69. 88), (29. 85. 8), (27. 79. 4), (25. 73. 98), (33. 77. 6), (21. 61. 90), (45. 81. 14), (51. 83. 18), (15. 43. 78), (13. 37. 74), (11. 31. 70), (75. 91. 34), (7. 19.62), (87. 95. 42), (93. 97. 46), (58. 94. 96), (40.44.66), (10. 16. 26), (30.38. 82), (68.80.2), (22. 36. 92), (24. 54. 72), (12. 52. 86).

This method may be also represented as a one-step cycle. For if we denote the girls by a point $k$ at the centre of the circle, and points $a_{1}, b_{1}, a_{2}, b_{2}, a_{3}, b_{3}, \ldots$ placed in that order on the circumference, we can re-write the solutions in the suffix notation, and then the cyclical permutation of the numbers denoting the suffixes is by one step at a time.

The one-step and two-step methods described above cover 
all cases except those where $n$ is of the form $24 m+21$. These I have failed to bring under analogous rules, but we can solve them by recourse to the three-step cycles next described.

Three-Step Cycles. The fact that certain cases are soluble by one-step cycles, and others by two-step cycles, suggests the use of three-step cycles, and the fact that $n$ is a multiple of 3 points to the same conclusion. On the other hand, if we denote the $n$ girls by $1,2,3, \ldots n$, and make a cyclical permutation of three steps at a time (or if we denote the girls by $a_{1}, b_{1}, c_{1}, a_{2}, b_{2}, c_{2}, \ldots$, and make a cyclical permutation of the suffixes one step at a time), we cannot get arrangements for more than $n / 3$ days. Hence there will remain $(n-1) / 2-n / 3$ days, that is, $(n-3) / 6$ days, for which we have to find other arrangements. In fact, however, we can arrange the work so that in addition to the cyclical arrangements for $n / 3$ days we can find $(n-3) / 6$ single triplets from each of which by a cyclical permutation of the numbers or suffixes an arrangement for one of these remaining days can be obtained; other methods are also sometimes available.

For instance take the case of 21 girls. An arrangement for the first day is (1. 4. 10), (2.5.11), (3.6.12), (7. 14. 18), (8. 15. 16), (9. 13. 17), (19.20.21). From this by cyclical permutations of the numbers three steps at a time, we can get arrangements for 7 days in all. The arrangement for the 8th day can be got from the triplet (1.6.11) by a three-step cyclical permutation of the numbers in it. Similarly the arrangement for the 9 th day can be got from the triplet (2. 4. 12), and that for the 10th day from the triplet (3.5. 10), by three-step cyclical permutations.

This method was first used by A. Bray in 1883, and was subsequently developed by Dudeney and Eckenstein. It gives a solution for every value of $n$ except 15 , but it is not so easy to use as the methods already described, partly because the solution is in two parts, and partly because the treatment varies according as $n$ is of the form $18 m+3$, or $18 m+9$, or $18 m+15$. Most of the difficulties in using it arise in the case when $n$ is of the form $18 m+15$. 
The geometrical representation is sufficiently obvious. In the methods used by Legros and Eckenstein, previously described, the girls were represented by $2 y$ equidistant points on the circumference of a circle and a point at its centre. It is evident that we may with equal propriety represent all the girls by symbols placed at equidistant intervals round the circumference of a circle: such solutions are termed noncentral. The symbols may be $1,2,3, \ldots n$, or letters $a_{1}, b_{1}, c_{1}$, $a_{2}, b_{2}, c_{2}, \ldots$ Any triplet will be represented by a triangle whose sides are chords of the circle. The arrangement on any day is to include all the girls, and therefore the triangles representing the triplets on that day are $n / 3$ in number, and as each girl appears in only one triplet no two triangles can have a common vertex.

The complete three-step solution will require the determination of a system of $(n-1) / 2$ inscribed triangles. In the first part of the solution $n / 3$ of these triangles must be selected to form an arrangement for the first day, so that by rotating this arrangement three steps at a time we obtain triplets for $n / 3$ days in all. In the second part of the solution we must assure ourselves that the remaining $(n-3) / 6$ triangles are such that from each of them, by a cyclical permutation of three steps at a time, an arrangement for one of the remaining $(n-3) / 6$ days is obtainable.

As before we begin by tabulating the possible differences $[1],[2],[3], \ldots[(n-1) / 2]$, whose values denote the lengths of the sides $p, q, r$ of the possible triangles, also, we have either $p+q=r$ or $p+q+r=n$. From these values of $p, q, r$ are formed triads, and in these triads each difference must be used three times and only three times. Triangles of these types must be then formed and placed in the circle so that the side denoting any assigned difference $p$ must start once from a number of the form $3 m$, once from a number of the form $3 m+1$, and once from a number of the form $3 m+2$. Also an isosceles triangle, one of whose sides is a multiple of three, cannot be used: thus in any particular triad a $3,6,9, \ldots$ cannot appear more than once. Save in some exceptional cases of high values of $n$, every triangle, one of whose 
sides is a multiple of 3 , must be used in the first part of the solution. In the whole arrangement every possible difference will occur $n$ times, and, since any two assigned numbers can occur together only once, each difference when added to a number must start each time from a different number. I will not go into further details as to how these triangles are determined, but $I$ think the above rules will be clear if I apply them to one or two easy examples.

For 9 girls, the possible differences are [1], [2], [3], [4], each of which must be used three times in the construction of four triangles the lengths of whose sides $p, q, r$ are such that $p+q=r$ or $p+q+r=9$. One possible set of triads formed from these numbers is $[1,2,3],[1,2,3],[2,3,4]$, and $[1,4,4]$. Every triangle with a side of the length [3] must appear in the first part of the solution; thus the triplets used in the first part of the solution must be obtained from the first three of these triads. Hence we obtain as an arrangement for the first day the triplets (1. 3. 9), (2.4.7), (5.6.8). From this, three-step cyclical permutations give arrangements for other two days. The remaining triad $[1,4,4]$ leads to a triplet (1. 2.6) which, by a three-step cyclical permutation, gives an arrangement for the remaining day.

If we use the suffix notation, an arrangement for the first day is $a_{1} b_{2} a_{8}, b_{1} c_{2} b_{3}, c_{1} a_{2} c_{3}$. From this, by simple cyclical permutations of the suffixes, we get arrangements for the second and third days. Lastly, the triplet $a_{1} b_{1} c_{1}$ gives, by cyclical permutation of the suffixes, the arrangement for the fourth day, namely, $a_{1} b_{1} c_{1}, a_{2} b_{2} c_{2}, a_{3} b_{3} c_{8}$.

For 15 girls, the three-step process is inapplicable. The explanation of this is that two triads are required in the second part of the solution, and in neither of them may a 3 appear. The triads are to be formed from the differences $[1],[2], \ldots$ [7], each of which is to be used three times, and the condition that in any particular triad only one 3 or one 6 may appear necessitates that six of the triads shall involve a 3 or a 6 . Hence only one triad will be available for the second part of the solution. 
I proceed to give one solution of this type for every remaining case where $n$ is less than 100. I give some in the numerical, others in the suffix notation. The results will supply an indication of the process used.

First, I consider those cases where $n$ is of the form $18 m+3$. In these cases it is always possible to find $2 m$ triads each repeated thrice, and one equilateral triad, and to use the equilateral and $m$ triads in the first part of the solution, the 3 triplets representing any one of these $m$ triads being placed in the circle at equal intervals from each other in the first day's arrangement. From this, three-step or one-step cyclical permutations give arrangements for $6 m+1$ days in all. In the second part of the solution each of the $m$ remaining triads is used thrice; it suffices to give the first triplet on each day, since from it the other triplets on that day are obtained by a three-step cyclical permutation.

For 21 girls an arrangement for the first day, for the first part of the solution, is $(1.4 .10),(8.11 .17),(15.18 .3),(2.6 .7),(9.13 .14),(16.20 .21),(5.12 .19)$. From this, three-step or one-step cyclical permutations give arrangements for 7 days in all. The firgt triplets used in the second part of the solution are (1. 3. 11), (2. 4. 12), (3. 5. 13). Each of these three triplets gives by a threestep cyclical permutation an arrangement for one of the remaining 3 days.

For 39 girls, arrangements for 13 days can be obtained from the following arrangement of triplets for the first day: (1. 4. 13), (14. 17. 26), (27. 30. 39), (2. 8. 23), (15. 21. 36), (28. 34. 10), (7.11. 12), (20.24. 25), (33. 37. 38), (3. 5. 19), (16. 18. 32), (29.31.6), (9.22. 35). From each of the 6 triplets (1.8.18), (2. 9. 19), (3. 10. 20), (1.9.20), (2. 10.21), (3. 11. 22), an arrangement for one of the remaining 6 days is obtainable.

For 57 girls, arrangements for 19 days can be obtained from the following arrangement of triplets for the first day: (1.4.25), (20. 23.44), (39.42.6), (2. 8. 17), (21. 27. 36), (40. 46. 55), (3. 15. 33), (22. 34. 52), (41.53. 14), (18. 19. 26), (37. 38. 45), (56. 57. 7), (13. 30. 35), (32. 49. 54), (51. 11. 16), (9. 29. 43), (28.48.5), (47. 10. 24), (12. 31. 50). From each of the 9 triplets (1. 3. 14), (2. 4. 15), (3. 5. 16), (1. 5. 30), (2. 6. 31), (3. 7. 32), (1. 11. 27), (2. 12. 28), (3. 13. 29), an arrangement for one of the remaining 9 days is obtainable.

For 75 girls, arrangements for 25 days can be obtained from the following arrangement of triplets for the first day: (1.4. 10), (26. 29. 35), (51. 54.60), (3. 15. 36), (28. 40.61), (53.65. 11), (2. 17. 41), (27. 42.66), (52. 67. 16), (13. 31. 58), (38. 56. 8), (63.6.33), (14. 21. 22), (39. 46. 47), (64. 71. 72), (7. 18. 20), (32. 43. 45), (57. 68. 70), (5. 9. 37), (30. 34. 62), (55. 59. 12), (19. 24. 50), (44. 49.75), (69.74. 25), (23. 48. 73). From each of the 12 triplets (1. 11. 30), (2. 12. 31), (3. 13. 32), (1. 15. 35), (2. 16. 36), (3. 17. 37), (1. 17. 39), (2. 18. 40), (3. 19.41), (1. 18. 41), (2. 19.42), (3. 20.43), an arrangement for one of the remaining 12 days is obtainable.

For 93 girls, arrangements for 31 days can be obtained from the following arrangement of triplets for the frst day: (1. 76. 79), (32.14. 17), (63. 45. 48), (13. 25. 55), (44. 56. 86), (75. 87. 24), (29. 50. 74), (60. 81. 12), (91. 19. 43), (20. 26. 59), (51. 57. 90), (82. 88. 28), (3. 30. 39), (34.61.70), (65.92. 8), (27. 64. 71), (58. 2. 9), (89. 33. 40), (35.36. 52), (66.67. 83), (4. 5. 21), (11. 15. 37), (42. 46. 68), (73. 77. 6), (16. 18. 41), (47. 49. 72), (78. 80. 10), 
(23. 31. 69), (54.62. 7), (85.93. 38), (22. 53. 84). From each of the 15 triplets (1. 14. 42), (2. 15. 43), (3. 16.44), (1.33.44), (2. 34.45), (3.35.46), (1. 11.30), (2. 12.31), (3.13. 32), (1. 6. 41), (2. 7.42), (3. 8. 43), (1. 15.35), (2. 16. 36), (3. 17. 37), an arrangement for one of the remaining 15 days is obtainable by a three-step cyclical permutation.

Before leaving the subject of numbers of this type I give two other solutions of the case when $n=21$, one to illustrate the ase of the suffix notation, and the other a cyclical solution which is uniquely three-step.

If we employ the suffix notation, the suffixes, with the type here used, are somewhat trying to read. Accordingly hereafter $I$ shall write $a 1, a 2, \ldots$, instead of $a_{1}, a_{2}, \ldots$. In the case of 21 girls, an arrangement for the first day is (a1. a2. a4), (b1.b2.b4), (c1.c2. c4), (a3.b6. c5), (b3.c6.a5), (c3. a6. b5), (a7. b7. c7). From this, by one-step cyclical permutations of the suffizes, we get arrangements for the $2 \mathrm{nd}, 3 \mathrm{rd}, 4 \mathrm{th}, 5 \mathrm{th}, 6 \mathrm{th}$ and 7 th days. The arrange. ment for the 8th day can be obtained from the triplet (a1.b2.c4) by permuting the suffixes cyclically one step at a time. Similarly the arrangement for the 9th day can be obtained from the triplet (b1. c2, a4) and that for the 10th day from the triplet (c1. a2,b4). Thus with seven suffixes we keep 7 for eqch symabol in one triplet, and every other triplet depends on one or other of only two arrangements, namely, (1. 2. 4), or (3.6.5). If the solution be written out at length the principle of the method used will be clear.

Cyclical solutions which are uniquely three-step can also be obtained for numbers of the form $18 m+3$; in them the same triads can be used as before, but they are not placed at equal intervals in the circle. I give 21 girls as instance. The arrangements on the first 7 days can be obtained from the arrangement (1. 4. 10), (2. 20. 14), (15. 18.3), (16. 17. 21), (8. 9. 13), (6. 7. 11), (5. 12. 19) by a three-step (but not by a one-step) oyclical permutation. From each of the triplets (1.3.11), (2.4.12), (3. 5. 13), an arrangement for one of the remaining 3 days is obtainable.

Next, I consider those cases where $n$ is of the form $18 m+9$. Here, regular solutions in the suffix notation can be obtained in all cases except in that of 27 girls, but if the same solutious are expressed in the numerical notation, the triads are irregular. Accordingly, except when $n=27$, it is better to use the suffix notation. I will deal with the case when $n=27$ after considering the cases when $n=45,63,81,99$.

For 45 girls, an arrangemest for the first day consists of the 5 triplets (a1. a12. a13), (a2.a9. a11), (a5. a10.b15), (a4.b3. c7), (a8. b6. c14), and the 10 analogous triplets, namely, (b1. 812.b13), (c1. c12.c13), (b2. 69.b11), (c2.c9. c11), (b5. b10.c15), (c5.c10.a15), (b4.c3.a7), (c4. a3. b7), (b8. c6. a14), (c8. a6. b14). From these, by one-step cyclical permutations of the suffixes, the arrangements for 15 days can be got. Each of the 2 triplets (c4. b3. a7), (c8.b6.a14), the 4 analogous triplets, namely, (a4.c3.b7), (b4.a3.c7), (a8.c6.b14), (b8. a6. c14), and the triplet (a1. b1. c1), gives, by a one-step cyclical permutation of the suffixes, an arrangement for one of the remaining 7 days.

For 63 girls, an arrangement for the first day consists of the 7 triplets (a1. a10. a9), (a5. a8. $a 3),(a 4 . a 19 . a 15),(a 7 . a 14 . b 21),(a 20 . b 11 . c 12)$, (a16. b13. c18), (a17. b2. c6), and the 14 anslogous triplets, From these, by 
one-step cyclical permutations of the suffixes, the arrangements for 21 days can be got. Each of the 10 triplets, consisting of the 3 triplets $(c 20 . b 11$. a12), (c16. b13, $a 18),(c 17 . b 2 . a 6)$, the 6 analogous triplets, and the triplet (a1. b1. $c 1)$, gives, by a one-step cyclieal permutation of the suffixes, an arrangement for one of the remaining 10 days.

For 81 girls, an arrangement for the first day consists of the 9 triplets (a5. a7. a8), (a3. a10. a14), (a11. a21. a26), (a4. a12. a25), (a9. a18. b27), (a22. b20.c19), (a24.b17.c13), (a16.b6.c1), (a23. b15.c2), and the 18 analogous triplets. From these, by cyclical permutations of the suffixes, the arrangements for 27 days can be got. Each of the 13 triplets consisting of the 4 triplets (c22.b20. a19), (c24.b17. a13), (c16.b6. a1), (c23. b15. a2), the 8 analogous triplets, and the triplet (a1. $b 1$. $c 1$ ), gives, by a cyclical permutation of the suffixes, an arrangement for one of the remaining 13 days.

For 99 girls, an arrangement for the first day consists of the 11 triplets (a1. a3. $a 10),(a 2 . a 6 . a 20)$, (a4. a12. $a 7)$, (a8. a24. a14), (a16. a15. a28), (a11. a22. b33), (a32. b30. c23), (a31. b27.c13), (a29.b21. c26), (a25. b9. c19), (a17. b18. $c 5$ ), and the 22 analogous triplets. From these, by cyclical permutations of the suffixes, the arrangements for 33 days can be got. Each of the 16 triplets consisting of the 5 triplets (c32. b30.a23), (c31.b27. a13), (c29.b21. a26), (c25. b9. a19), (c17. b18. a5), the 10 analogous triplets, and the triplet (a1. b1. c1), gives, by a cyclical permutation of the suffixes, an arrangement for one of the remaining 16 days.

For 27 girls, an arrangement of triplets for the first day is (1.7.10), (14.17.8), (27.6. 9), (13. 20. 25), (11.23. 18), (12.16.24), (21. 2.4), (15. 22. 26), (19.3.5). From this, three-step cyclical permutations give arrangements for 9 days in all. The first triplets on the remaining 4 days are (1. 2. 3), (1.6. 20), (1.11. 15), (1. 17. 27), from each of which, by a three-step cyclical permutation, an arrangement for one of those days is obtainable.

Regular solutions in the numerical notation can also be obtained for all values of $n$, except 9 , where $n$ is of the form $18 m+9$. I give 27 girls as an instance. The first day's arrangement is (1.2.4), (10.11. 13), (19. 20.22), (8. 15. 23), (17. 24. 5), (26. 6. 14), (3. 25. 9), (12. 7. 18), (21. 16. 27); from this, arrangements for 9 days in all are obtained by one-step cyclical permutations. Each of the three triplets (1. 5.15), (2.6.16), (3. 7. 17) gives an arrangement for one day by a three-step cyclical permutation. Finally the triplet (1. 10. 19), represented by an equilatexal triangle, gives the arrangement on the last day by a one-step cyclical permutation.

Lastly, I consider those cases where $n$ is of the form $18 m+15$. As before, the solution is divided into two parts. In the first part, we obtain an arrangement of triplets for the first day, from which arrangements for $6 m+5$ days are obtained by three-step cyclical permutations. In the second part, we obtain the first triplet on each of the remaining $3 m+2$ days, from which the other triplets on that day are obtained by three-step eyclical permutations.

For 33 girls, an arrangement in the first part is (1. 13. 19), (23.11. 5), (3. 15. 21), (2. 4. 7), (12.14.17), (28. 30. 33), (6.16.25), (10.20.29), (8. 18.27), (24. 31. 32), (9. 22. 26). The triplets in the second part are (25. 32. 33), (26. 33. 1), (10. 23. 27), (11. 24. 28), (1. 12. 23). 
For 51 girls, an arrangement in the first part is (17. 34. 51), (49.16.10), (11. 44. 50), (15. 33. 27), (19.4.46), (2.38.29), (21.36.45), (22. 25. 24), (5.8.7), $(39.42 .41),(43.13 .20),(26.47 .3),(9.30 .37),(1.14 .6),(35.48 .40),(18.31 .23)$, (28. 32. 12). The triplets in the second part are (29. 33. 13), (30.34. 14), (1. 26. 12), (2. 27. 13), (3. 28.14), (1. 11. 30), (2. 12. 31), (3. 13. 32).

For 69 girls, an arrangement in the first part is (23. 46. 69), (31. 34. 43), (11. 8. 68), (54. 57. 66), (58. 52. 28), (35. 29. 5), (45. 51. 6), (16. 1. 49), (62. 47. 26), (39. 24. 3), (4. 55. 42), (50. 32. 19), (27. 9. 65), (40.67. 18), (17. 44. 64), (63. 21. 41), (10.14. 15), (33. 37. 38), (56.60.61), (25.53. 36), (2. 30. 13), (48. 7. 59), (22. 20. 12). The triplets in the second part are (23. 21. 13), (24.22. 14), (1.8.33), (2.9.34), (3.10.35), (1. 17. 36), (2.18.37), (3. 19. 38), (1. 41. 15), (2. 42. 16), (3. 43. 17).

For 87 girls, an arrangement in the first part is (29.58.87), (76. 82. 73), (47. 53. 44), (15. 9. 18), (70. 1. 13), (41. 59. 71), (12. 30.42), (4. 67. 31), (2. 26. 62), (60.84. 33), (40.79. 25), (11.50.83), (69. 21. 54), (43.64. 63), (14. 35. 34), (72. 6. 5), (61. 16. 77), (32. 74. 48), (3. 45. 19), (28. 68. 66), (37. 86. 39), (10. 8. 57), (46. 80. 36), (22. 65. 75), (7. 17. 51), (85. 23. 78), (52. 20. 27), (49. 56. 81), (55. 38. 24). The triplets in the second part are (56. 39. 25), (57. 40.26), (1.5.42), (2.6.43), (3. 7.44), (1. 29.6), (2.30.7), (3. 31. 8), (1. 9. 20), (2. 10.21), (3. 11.22), (1. 14. 36), (2. 15. 37), (3.16. 38).

Before proceeding to the consideration of other methods I should add that it is also possible to obtain irregular solutions of cases where $n$ is of any of these three forms. As an instance I give a three-step solution of 33 girls. A possible arrangement in the first part is (1. 4. 10), (14. 23. 26), (9. 15. 30), (3. 28. 33), (2. 6. 8), (11. 18. 27), (13. 24. 25), (5. 16. 20), (7. 17. 22), (21. 29. 31), (12. 19. 32). The triplets in the second part are (1. 2. 21), (1. 8. 30), (1. 3. 26), (1. 15. 20), (1. 17. 18). I describe this solution as irregular, since all, save one, of the triads used are different.

The Focal Method. Another method of attacking the problem, comparatively easy to use in practice, is applicable when $n$ is of the form $24 m+3 p$, where $p=6 q+3$. It is due to Eckenstein. Here it is convenient to use a geometrical representation by denoting $24 m+2 p$ girls by equidistant numbered points on the circumference of a circle, and the remaining $p$ girls by lettered points placed inside the circle; these $p$ points are termed foci. The solution is in two parts. In the first part, we obtain an order from which the arrangements for $12 m+p$ days are deducible by a two-step cycle of the numbers: in none of these triplets does more than one focus appear. In the second part, we find the arrangements for the remaining $3 q+1$ days; here the foci and the numbered points are treated separately, the former being 
arranged by any of the methods used for solving the case of $6 q+3$ girls, while of the latter a typical triplet is used on each of those days, from which the remaining triplets on that day are obtained by cyclical permutations.

This method covers all cases except when $n=15,21,39$; and solutions by it for all values of $n$ less than 200 have been written out. Sets of all the triplets required can be definitely determined. One way of doing this is by finding the primitive roots of the prime factors of $4 m+2 q+1$, though in the simpler cases the triplets can be written down empirically without much trouble. An advantage of this method is that solutions of several cases are obtained by the same work. Suppose that we have arranged suitable triangles in a circle, having on its circumference $12 m+p$ or $3 c$ equidistant points, and let $y$ be the greatest integer satisfying the indeterminate equation $2 x+4 y+1=c$, where $x=0$ or $x=1$, and $a$ the highest multiple of 6 included in $x+y$, then solutions of not less than $y+1-\alpha$ cases can be deduced. Thus from a 27 circle arrangement where $c=9, y=2, x=0, \alpha=0$, we can by this method deduce three solutions, namely when $n=57,69,81$; from a 39 circle arrangement where $c=13, y=3, x=0, \alpha=0$, we can deduce four solutions, namely when $n=81,93,105,117$.

I have no space to describe the method fully, but $I$ will give solutions for two cases, namely for 33 girls $(n=33, m=1, p=3)$ where there are 3 foci, and for 51 girls $(n=51, m=1, p=9)$ where there are 9 foci.

For 33 girls we have 3 foci which we may denote by $a, b, c$, and 30 points which we may denote by the numbers 1 to 30 placed at equidistant intervals on the circumference of a circle. Then if the arrangement on the first day is (a. 5.10), (b. 20.25), (c. 15.30), (1. 2. 14), (16.17.29), (4. 23. 26), (19.8.11), (9.7.3), (24.22.18), (6.27.13), (21.12.28), a two-step cyclical permutation of the numbers gives arrangements for 15 days; that on the second day being (a. 7. 12), (b. 22. 27), \&c. The arrangement on the 16th day is $(a . b . c),(1.11 .21),(2.12 .22)$, (3. 13. 23), ... (10. 20. 30).

For 51 girls we have 9 foci which we may denote by $a, b, c$, 
$d, e, f, g, h, j$, and 42 points denoted by $1,2, \ldots 42$, placed at equidistant intervals on the circumference of a circle. Then if the arrangement on the first day is $(a .5 .6),(b .26,27),(c .3 .10)$, (d. 24. 31), (e.19.34), (f. 40.13), (g. 39.16), (h. 18. 37), (j. 21.42), (9. 11. 22), (30.32.1), (35.41. 2), (14. 20.23), (17.29.12), (38. 8. 33), (7. 15. 25), (28. 36. 4), a two-step cyclical permutation of the numbers gives arrangements for 21 days. Next, arrange the 9 foci in triplets by any of the methods already given so as to obtain arrangements for 4 days. From the numbers 1 to 42 we can obtain four typical triplets not already used, namely (1. 5. 21), (2.6. 22), (3. 7. 23), (14. 28. 42). From each of these triplets we can, by a threestep cyclical permutation, obtain an arrangement of the 42 girls for one day, thus getting arrangements for 4 days in all. Combining these results of letters and numbers we obtain arrangements for the 4 days. Thus an arrangement for the first day would be (a.c.j), (b. d.g), (e.f. h), (1. 5. 21), (4. 8. 24), (7. 11. 27), (10. 14.30), (13.17.33), (16.20.36), (19.23. 39), (22. 26. 42), (25. 29. 3), (28. 32.6), (31. 35.9), (34. 38. 12), (37. 41. 15), (40.2.18). For the second day the corresponding arrangement would be $(d . f . c),(e . g . a),(h . j . b),(2.6 .22)$, (5. 9. 25), \&c.

Analytical Methods. The methods described above, under the headings One-Step, Two-Step and Three-Step Cycles, involve some empirical work. It is true that with a little practice it is not difficult to obtain solutions by them when $n$ is a low number, but the higher the value of $n$ the more troublesome is the process and the more uncertain its success. A general arithmetical process has, however, been given by which it is claimed that some solutions for any value of $n$ can be always obtained. Most of the solutions given earlier in this chapter can be obtained in this way.

The essential feature of the method is the arrangement of the numbers by which the girls are represented in an order such that definite rules can be laid down for grouping them in pairs and triplets so that the differences of the numbers in each pair or triplet either are all different or are repeated as often as 
may be required. The process depends on finding primitive roots of the prime factors of whatever number is taken as the base of the solution. When $(n-1) / 2$ is prime, of the form $6 u+1$, and is taken as base, the order is obtainable at once, and the rules for grouping the numbers are easy of application; owing to considerations of space I here confine myself to such instances, but similar though somewhat longer methods are applicable to all cases.

I use the geometrical representation already explained. We have $n=2 y+1$, and $y$ is a prime of the form $6 u+1$. In forming the triplets we either proceed directly by arranging all the points in threes, or we arrange some of them in pairs and make the selection of the third point dependent on those of the two first chosen, leaving only a few triplets to be obtained otherwise. In the former case we have to arrange the numbers in triplets so that each difference will appear twice, and so that no two differences will appear together more than once. In the latter case we have to arrange the numbers so that the differences between the numbers in each pair comprise consecutive integers from 1 upwards and are all different. In both cases, we commence by finding a primitive root of $y$, say $x$. The residues to the modulus $y$ of the $6 u$ successive powers of $x$ form a series of numbers, $e 1, e 2, e 3, \ldots$, comprising all the integers from 1 to $6 u$, and when taken in the order of the successive powers, they can be arranged in the manner required by definite rules.

I will apply the method to the case of 27 girls from which the general theory, in the restricted case where $y$ is a prime of the form $6 u+1$, will be sufficiently clear. In this case we have $n=27, y=13, u=2$, and $x=2$. I take 13 as the base of the analysis. I will begin by pairing the points, and this being so, it is convenient to represent the girls by a point $k$ at the centre of a circle and points $a 1, b 1, a 2, b 2, \ldots$ at equidistant intervals on the circumference. We reserve $k, a 13$, 613 for one triplet, and we have to arrange the other 24 points so as to form 8 triangles of certain types. The residues are in the order $2,4,8,3,6,12,11,9,5,10,7,1$, and these may be taken as the suffixes of the remaining ' $a$ 's and ' $b$ 's. 
First arrange these residues in pairs so that every difference between the numbers in a pair occurs once. One rule, by which this can be effected, is to divide the residues into two equal sections and pair the numbers in the two sections. This gives (2. 11), (4. 9), (8.5), (3. 10), (6.7), (12.1) as possible pairs. Another such rule is to divide the residue into six equal sections, and pair the numbers in the frst and second sections, those in the third and fourth sections, and those in the fifth and sixth sections. This gives (2.8), (4. 3), (6. 11), (12.9), (5. 7), (10.1) as possible pairs. Either arrangement can be used, but the first set of pairs leads only to scalene triangles. In none of the pairs of the latter set does the sum of the numbers in a pair add up to 13, and since this may allow the formation of isosceles as well as of scalene triangles, and thus increase the variety of the resulting solutions, I will use the latter set of pairs. We use these basic pairs as suffixes of the ' $a$ 's, and each pair thus determines two points of one of the triangles required. We have now used up all the ' $a$ 's. The third point associated with each of these six pairs of points must be $a$ ' $b$, and the remaining six ' $b$ 's must be such that they can be arranged in suitable triplets.

Next, then, we must arrange the $6 u$ residues $e 1, e 2, e 3, \ldots$ in possible triplets. To do this arrange them cyclically in triplets, for instance, as shown in the first column of the left half of the annexed table. We write in the second column the differences between the first and second numbers in each triplet, in the third column the differences between the second and third numbers in each triplet, and in the fourth column the differences between the third and first numbers in each triplet. If any of these differences $d$ is greater than $3 u$ we may replace it by the complementary number $y-d$ : that this is permissible is obvious from the geometrical representation. By shifting cyclically the symbols in any vertical line in the first column we change these differences. We can, however, in this way always displace the second and third vertical lines in the first column so that the numbers in the second, third, and fourth columns include the numbers 1 to $3 u$ twice over. This can be effected 
thus. If any term in the residue series is greater than $3 u$ replace it by its complementary number $y-e$. In this way, from the residue series, we get a derivative series $d 1, d 2, d 3, \ldots$ such that any $3 u$ consecutive terms comprise all the integers from 1 to $3 u$. The first half of this series may be divided into three equal divisions thus: (1) $d 1, d 4, d 7, \ldots$; (2) $d 2, d 5, d 8, \ldots$; (3) $d 3, d 6, d 9, \ldots$ If the displacement is such that the first numbers in the second, third, and fourth columns are contained in different divisions, each difference must occur twice, and it will give a possible solution. Other possible regular arrangements give other solutions. Applying this to our case we have the residue series, $2,4,8,3,6,12,11,9,5,10,7,1$. The derivative series is $2,4,5,3,6,1,2,4,5,3,6,1$. The three divisions are (i) 2,3 ; (ii) 4,6 ; (iii) 5,1 . The cyclical arrangement we started with and the consequent differences are shown in the left half of the accompanying table. A cyclical change

\begin{tabular}{|rr|r|r|r|rr|r|r|r|}
\hline 2.4. & 8 & 2 & 4 & 6 & 2.6. & 5 & 4 & 1 & 3 \\
3.6. & 12 & 3 & 6 & 4 & 3.9. & 1 & 6 & 5 & 2 \\
11.9. & 5 & 2 & 4 & 6 & 11.7. & 8 & 4 & 1 & 3 \\
10.7. & 1 & 3 & 6 & 4 & 10.4 .12 & 6 & 5 & 2 \\
\hline
\end{tabular}

as described above of the vertical lines of the symbols in the first column gives the arrangement in the right half of the table. Here each difference occurs twice and accordingly this gives a possible arrangement of the triplets, namely, 2, 6, 5; $3,9,1 ; 11,7,8 ; 10,4,12$. These are the suffixes of the ' $b$ 's. We have now to use six of these ' $b$ 's in connection with the basic ' $a$ 's already determined, keeping the other six ' $b$ 's for the remaining two triangles.

For instance we may obtain a scalene solution by taking as a suffix of the ' $b$ ' associated with any pair of ' $a$ 's, a number equal to the sum of the suffixes of the ' $a$ 's. We thus get as a solution ( $a 2 . a 8 . b 10),(a 4 . a 3 . b 7),(a 6 . a 11 . b 4),(a 12 . a 9 . b 8)$, (a5. a7. b12), (a10. a1. b11), (b2. b6. b5), (b3.b9.b1), (l.a13.b13). Or we might take as a suffix of the ' $b$ ' associated with any pair of ' $a$ 's, the number midway between the suffixes 
of the ' $a$ ' $s$ on the 13 circle. This gives the following solution, in which the first six triangles are isosceles: $(a 2 . a 8 . b 5)$, (a4. a3. b10), (a6. a11. b2), (a12. a9. b4), (a5. a7. b6), (a10. a1. b12), (b3. b9.b1), (b11. b7. b8), (k. a13.b13).

In the case of 27 girls, we may equally well represent the points by $k$ at the centre of the circle and 26 equidistant points $1,2, \ldots 26$ on the circumference. The points previously denoted by $a$ and $b$ with the suffix $h$ are now denoted by the numbers $2 h-1$ and $2 h$. Hence the basic pairs (2. 8), (4. 3), ... become (3. 15), (7. 5), (11. 21), (23. 17), (9.13), (19.1), and the corresponding scalene arrangement for the first day is (3.15.20), (7. 5. 14), ... (k. 25. 26). From this by a two-step cyclical permutation of the numbers, an arrangement for 13 days can be got.

The case of 27 girls can also be treated by the direct formation of triplets. The triplets must be such that each difference is represented twice, but so that the groups of differences are different. There are analytical rules for forming such triplets somewhat analogous to those $I$ have given for forming basic pairs, but their exposition would be lengthy, and $I$ will not discuss them here. One set which will answer our purpose is (1.12.5), (2.3.10), (4.6. 9), (8.11.7), giving respectively the differences $[2,6,4],[1,6,5],[2,3,5],[3,4,1]$. Now every difference $d$ in a 13 circle will correspond to $d$ or $13-d$ in a 26 circle, and every residue $e$ in a 13 circle will correspond to $e$ or $13+e$ in a 26 circle. Further, a triplet in the 26 circle must either have three even differences, or one even and two odd differences. Hence from the above sets we can get the following arrangement for the first day, (1.25.5) and (14.12.18) with differences $[2,6,4],(15.3 .10)$ and (2.16.23) with differences $[12,7,5],(17.6 .9)$ and $(4.19 .22)$ with differences $[11,3,8],(21.11 .20)$ and (8. 24. 7) with differences $[10,9,1]$, and (k.13.26). From this by either a one-step or a two-step cyclical permutation of the numbers, an arrangement for 13 days can be got. I will not go into further details about the deduction of other similar solutions. A similar method is always applicable when $n$ is of the form $24 m+3$. 
The process by pairing when $y$ is a prime of the form $6 u+1$ is extremely rapid. For instance, in the case of 15 girls we have $n=15, y=7, u=1, x=5$. The order of the residues is $5,4,6,2,3,1$. By our rule we can at once arrange basic pairs (5. 4), (6. 2), (3. 1). From these pairs we can obtain numerous solutions. Thus using scalene triangles as above explained, we get as an arrangement for the first day $(a 5, a 4, b 2),(a 6 . a 2.61)$, (a3. a1.b4), (b3.b5.b6), (k.a7.b7), from which by a one-step cyclical permutation of the numbers, arrangements for the seven days can be obtained. Using the basic pairs as bases of isosceles triangles, we get as an arrangement for the first day (a5. a4.b1), (a6.a2.b4), (a3.a1.b2), (b3.b5.b6), (k.a7.b7).

Again, take the case of 39 girls. Here we have $n=39$, $y=19, u=3, x=3$. The order of the residues is $3,9,8 ; 5,15,7$; $2,6,18 ; 16,10,11 ; 14,4,12 ; 17,13,1$. The basic pairs are (3. 5), (9.15), (8. 7), (2. 16), \&c. These are the suffixes of the ' $a$ 's. The possible triplets which determine what ' $b$ 's are to be associated with these, and what ' $b$ 's are to be left for the remaining three triangles, can be determined as follows: From the residue series we obtain the derivative series $3,9,8,5,4,7,2,6,1$, \&c. The divisions are (i) $3,5,2$; (ii) $9,4,6$; (iii) $8,7,1$. A cyclical arrangement like that given above leads to the result in the left half of the annexed table which does not satisfy our condition. A cyclical displacement of the symbols in the vertical lines in the first column leads to the arrangement given in the right half of the table, and shows

\begin{tabular}{|c|c|c|c|c|c|c|c|}
\hline 3. 9.8 & 6 & 2 & 5 & 3. 15.18 & 7 & 3 & 4 \\
\hline 5. 15.7 & 9 & 8 & 2 & 5. 6.11 & 1 & 5 & 6 \\
\hline 2. 6. 18 & 4 & 7 & $\mathbf{3}$ & 2. 10.12 & 8 & 2 & 9 \\
\hline 16. 10. 11 & 6 & 1 & 5 & 16. 4. 1 & 7 & 3 & 4 \\
\hline 14. 4. 12 & 9 & 8 & 2 & 14. 13. 8 & 1 & 5 & 6 \\
\hline 17. 13. 1 & 6 & 7 & 3 & 17. 9.7 & 8 & 2 & 9 \\
\hline
\end{tabular}

that (3.15.18), (5.6.11), \&c. are possible triplets. From these results numerous solutions can be deduced in the same way as above. For instance one solution is (a3. a 5. b4), 
(a9. $a 15 . b 12),(a 8 . a 7 . b 17),(a 2 . a 16 . b 9),(a 6 . a 10 . b 8)$, (a18. a11.b5), (a14.a17.b6), (a4.a13.b18), (a12.a1.b16), (b3.b10.b1), (b2.b13.b7), (b14.b15.b11), (k.a19.b19).

In the case of 39 girls we may also extend the method used above by which for 27 girls we obtained the solution (1. 25. 5), (14. 12. 18),... We thus get a solution for 39 girls as follows: (1.25. 18), (14.38.31), (27. 12.5); (15. 16. 10), (28. 29. 23), (2. 3. 36); (17. 19. 35), (30. 32. 9), (4.6. 22); (21. 24. 33), (34. 37. 7), (8. 11. 20); (13. 26. 39). From this the arrangements for the first 13 days are obtained either by a one-step or a three-step cyclical permutation of the numbers. The single triplets from each of which an arrangement for one of the other six days is obtainable are (1. 5. 15), (2. 6. 16), (3. 7. 17); (1. 9. 20), (2. 10. 21), (3. 11. 22). From each of these the arrangement for one day is obtainable by a three-step cyclical permutation of the numbers.

These examples of the nse of the Focal and Analytical Methods are given only by way of illustration, but they will serve to suggest the applications to other cases. When the number taken as base is composite, the formations of the series used in the Analytical Method may be troublesome, but the principle of the method is not affected, though want of space forbids my going into further details. Eckenstein, to whom the development of this method is mainly due, can, with the aid of a table of primitive roots and sets of numbers written on cards, within half an hour obtain a solution for any case in which $n$ is less than 500 , and can within one hour obtain a solution for any case in which $n$ lies between 500 and 900 .

Number of Solutions. The problem of 9 girls has been subjected to an exhaustive examination. The number of solutions is 840 , if an arrangement on Monday, Tuesday, Wednesday and Thursday, and the same arrangement on (say) Monday, Tuesday, Thursday and Wednesday are regarded as identical. [If they are regarded as different the number of possible independent solutions is 20,160.] Any of these 840 solutions can however be deduced from any other of them by interchanges, and thus there is only one fundamental solution. The 
total number of possible arrangements of the girls in triplets for four days is $(280)^{4} /(4 !)$; hence the probability of obtaining a solution by a chance arrangement is about 1 in 300,000 .

In the case of 15 girls the number of solutions is said to be $65 \times(13 !)$, but I do not vouch for the correctness of this result. The number of fundamental solutions has not yet been definitely ascertained, but it has been shown that it is not less than seven and not more than eleven. The total number of ways in which the girls can walk out for a week in triplets is $(455)^{7}$; so the probability that any chance way satisfies the condition of the problem is very small.

Harison's Theorem. If we know solutions for Kirkman's Problem for $3 l$ girls and for $3 m$ girls we can find a solution for $3 l m$ girls. The particular case of this when $l=1$ was established by Walecki and given in the earlier editions of this work. Harison's proof, given in 1916, of the more general theorem is as follows:-

If the school-girls be denoted by the consecutive numbers from 1 to $3 l m$ and the numbers be divided into $3 l$ sets, each of $m$ consecutive numbers, each of these sets can, by the method for the $3 l$ problem, be divided in $(3 l-1) / 2$ collections of groups of three sets, so that every set shall be included once in the same group with every other set.

In one of these collections, each group of three sets (involving $3 \mathrm{~m}$ numbers) can, by the method for the $3 \mathrm{~m}$ problem, be arranged in triplets for $(3 m-1) / 2$ days, so as to have each number in each of the sets composing the group included once in the same triplet with every other number in the set to which it belongs and with every number in the other two sets in the group. This will give arrangements of all the numbers for that number of days.

In the remaining $(3 l-3) / 2$ collections, each group can be arranged in triplets for $m$ days, so as to have each number in each of the sets composing the group included once in the same triplet with each number of the other two sets. In the first arrangement in each collection, the first triplet in each group is composed of the first number of each set. In the second arrangement, the first triplet is composed of the first 
number of the first set, the second number of the second set, and the last number of the third set. In every other arrangement the first triplet is formed from the first triplet in the next proceeding arrangement, by adding unity to the number of the second set, subtracting unity from the number of the third set, and leaving the number of the first set unchanged. In every arrangement the second and all subsequent triplets are formed cyclically from the next preceding triplet.

The arrangements thus made of the numbers in all the groups of all the collections will give arrangements of all the numbers for $(3 l m-1) / 2$ days, and will provide a solution of the problem.

Harison's Theorem provides solutions, alternative to those given above, for the cases when $n=27,45,63,75,81,99$. Also we can, by it, from the solutions already given for all values of $n$ less than 100 , at once deduce solutions for the cases when $n=105,117,135,147,153,165,171,189,195$, \&c.

Extension to $n^{2}$ Girls. Peirce suggested the corresponding problem of arranging $n^{2}$ girls in $n$ groups, each group containing $n$ girls, on $n+1$ days so that no two girls will be together in a group on more than one day. We may conveniently represent the girls by a point $k$ at the centre of a circle and $n^{2}-1$ equidistant points, numbered $1,2,3, \ldots$, on the circumference.

When $n=2$, we may arrange initially the 4 points in two pairs, one pair consisting of $k$ and one of the points, say, 3 , and the other pair of the remaining points (1. 2). These two pairs give the arrangement for the first day. From them, the solution for the other days is obtained by one-step cyclical permutations.

When $n=3$, we may arrange initially the 9 points in three triplets, namely, $k$ and the ends of a diameter ( $k$. 4. 8); a triangle (1. 2.7); and the similar triangle (5.6.3) obtained by a four-step cyclical permutation. These three triplets give an arrangement for the first day. From them the solutions for the other days are obtained by one-step cyclical permutations.

When $n=4$, we may arrange initially the 16 points in four quartets, namely, $k$ and three equidistant points, $(k .5 .10 .15)$; 
a quadrilateral (1.2.4.8); and the two similar quadrilaterals (6. 7. 9. 13) and (11. 12. 14. 3) obtained by five-step cyclical permutations. These four quartets give an arrangement for the first day. From them the solutions for the other days are obtained by one-step cyclical permutations.

When $n=5$, we may arrange initially the 25 points in five quintets, namely, $k$ and four equidistant points, $(k .6 .12 .18 .24)$; a pentagon (2. 3. 5. 13. 22); and the three similar pentagons (8. 9. 11. 19. 4), (14. 15. 17. 1. 10), (20. 21. 23. 7. 16) obtained by six-step cyclical permutations. These five quintets give an arrangement for the first day. From them the solutions for the other days are obtained by one-step cyclical permutations. There is a second solution (k. 6. 12.18.24), (1.2.15.17.22), \&c.

Hitherto the case when $n=6$ has baffled all attempts to find a solution.

When $n=7$, we may initially arrange the 49 points in seven groups, namely ( $k$. 8. 16. 24. 32. 40. 48), a group (1. 2. 5. 11. 31. 36. 38) and five similar groups obtained by successive additions of 8 to these numbers. There are three other solutions: in these the second group is either (2. 3. 17. 28. 38. 45. 47), or (3. 4. 6. 18. 23. 41. 45), or (3. 4. 14. 17. 26. 45. 47).

When $n=8$ there are three solutions. If the first group is (k. 9. 18. 27. 36. 45. 54,63), the second group is either (1. 2. 4. 8. 16. 21. 32. 42), or (2. 3. 16. 22. 24. 50.55.62), or (3. 4. 7. 19. 24. 26. 32. 56): the other groups in each solution being obtained by successive addition of 9 to the numbers in the second group.

When $n=9$, one solution is as follows. Divide the girls into nine classes, each containing nine girls, denoted by $a 1, a 2, \ldots a 9$; $b 1, b 2, \ldots b 9$; and similarly for the letters $c, d, e, f, g, h, l$. The arrangement on the first day is made up of the a group, the $b$ group, etc. The arrangement on the second day is of nine groups as follows: first group, $k 1, a 5, b 3, c 8, d 7, e 9, f 4, g 6, h 2$; second group, $k 2, a 3, b 6, c 4, d 1, e 8, f 9, g 5, h 7$; third group, $k 3, a 8, b 4, c 7, d 5, e 2, f 1, g 9, h 6$; fourth group, $l k 4, a 7, b 1, c 5, d 8$, $e 6, f 3, g 2, h 9$; fifth group, $k 5, a 9, b 8, c 2, d 6, e 1, f 7, g 4, h 3$; 
sixth group, $k 6, a 4, b 9, c 1, d 3, e 7, f 2, g 8, h 5$; seventh group, $k 7, a 6, b 5, c 9, d 2, e 4, f 8, g 3, h 1$; eighth group, $k 8, a 2, b 7, c 6$, $d 9, e 3, f 5, g 1, h 4$; ninth group, $k 9, a 1, b 2, c 3, d 4, e 5, f 6, g 7, h 8$. The arrangements for the other eight days are got by shifting the numbers attached to the letters $a, b, \ldots h$ (but not $k$ ) in each group cyclically. Thus the first group on the third day will be, $k 1, a 3, b 8, c 7, d 9, e 4, f 6, g 2, h 5$.

When $n$ is composite no general method of attacking the problem has been discovered, though solutions for various particular cases have been given. But when $n$ is prime, we can proceed thus. Denote the $n^{2}$ girls by the suffixed letters shown

\begin{tabular}{|c|c|}
\hline 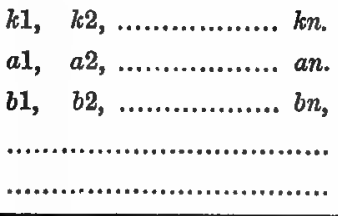 & 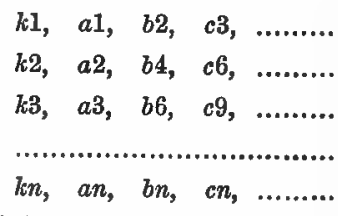 \\
\hline Arrangement on First Day & Arrangement on Second Day \\
\hline
\end{tabular}

in the left half of the above table. Take this as giving the arrangement on the first day. Then on the second day we may take as an arrangement that shown in the right half of the table. From the arrangement on the second day, the arrangements for the other days are obtained by one-step cyclical permutations of the suffixes of $a, b$, \&c. ; the suffixes of $k$ being unaltered.

Kirkman's Problem in Quartets. The problem of arranging $4 m$ girls, where $m$ is of the form $3 n+1$, in quartets to walk out for $(4 m-1) / 3$ days, so that no girl will walk with any of her school-fellows in any quartet more than once has been attacked. Methods similar to those given above are applicable, and solutions for all cases where $m$ does not exceed 49 have been written out. One example will suffice: for 16 girls (i.e. when $m=4$, $n=1$ ), arrangements in quartets for five days can be obtained from the following arrangement for the first day, (k. 5. 10. 15), (8. 2. 1. 4), (13. 7. 6. 9), (3. 12. 11. 14); from which three-step cyclical permutations of the numbers give arrangements for the 
other days. I conjecture that similar methods are applicable to corresponding problems about quintets, sextets, \&c.

Bridge Problem. Another analogous question is wherewe deal with arrangements in pairs instead of triplets. One problem of this kind is to arrange $4 m$ members of a bridge club for $4 m-1$ rubbers so that (i) no two members shall play together as partners more than once, and (ii) each member shall meet every other member as opponent twice. The general theory has been discussed by E. H. Moore, O. Eckenstein and G. A. Miller. A typical method for obtaining cyclic solutions is as follows. Denote the members by a point $k$ at the centre of a circle and by $4 m-1$ equidistant points, numbered $1,2,3, \ldots$, on the circumference. We can join the points $2,3,4, \ldots$ by chords, and these chords with (k. 1) give possible partners at the $m$ tables in the first rubber. A one-step cyclical permutation of the numbers will give the arrangements for the other rubbers if, in the initial arrangement, (i) the lengths of the chords representing every pair of partners are unequal and thus appear only once, and (ii) the lengths of the chords representing every pair of opponents appear only twice. Since the chords representing pairs of partners are unequal, their lengths are uniquely determined, but the selection of the chords is partly empirical. Solutions for many values of $m$ have been given. In the following examples for $m=2,3,4$, I give an arrangement of the card tables for the first rubber: the arrangements for the subsequent rubbers being thence obtained by one-step cyclical permutations of the numbers. If $m=2$, such an initial arrangement is ( $k .1$ against 5. 6) and (2. 4 against 3. 7). If $m=3$, one such initial arrangement is (k. 1 against 5.6), (2.11 against 3.9) and (4.8 against 7. 10). If $m=4$, one such initial arrangement is ( $k .1$ against 6. 11), (2. 3 against 5. 9), (4. 12 against 13.15), and (7. 10 against 8. 14). There are also solutions by other methods.

Sylvester's Corollary. To the original theorem J. J. Sylvester added the corollary that the school of 15 girls could walk out in triplets on 91 days until every possible triplet had walked abreast once, and he published a solution in 1861.

The generalized problem of finding the number of ways in 
which $x$ girls walking in rows of $a$ abreast can be arranged so that every possible combination of $b$ of them may walk abreast once and only once is as yet unsolved. Suppose that this number of ways is $y$. It is obvious that, if all the $x$ girls are to walk out each day in rows of $a$ abreast, then $x$ must be an exact multiple of $a$ and the number of rows formed each day is $x / a$. If such an arrangement can be made for $z$ days, then we have a solution of the problem to arrange $x$ girls to walk out in rows of $a$ abreast for $z$ days so that they all go out each day and so that every possible combination of $b$ girls may walk together once and only once. For instance, if $x=2 n, a=2, b=2$, we have $y=n(2 n-1) / 2, z=2 n-1$. If $x=15, a=3, b=2$, we have $y=35$; and these 35 rows can be divided into 7 sets, each of which contains all the symbols; hence $z=7$. 


\section{CHAPTER XI.}

\section{MISCELLANEOUS PROBLEMS.}

I propose to discuss in this chapter the mathematical theory of a few common mathematical amusements and games. I might have dealt with them in the first four chapters, but, since most of them involve mixed geometry and algebra, it is rather more convenient to deal with them apart from the problems and puzzles which have been described already; the arrangement is, however, based on convenience rather than on any logical distinction.

The majority of the questions here enumerated have no connection one with another, and I jot them down almost at random.

I shall discuss in succession the Fifteen Puzzle, the Tower of Hanö̈, Chinese Rings, and some miscellaneous Problems connected with a Pack of Cards.

The Fifteen Puzzle*. Some years ago the so-called Fifteen Puzzle was on sale in all toy-shops. It consists of a shallow wooden box-one side being marked as the top-in the form of a square, and contains fifteen square blocks or counters numbered $1,2,3, \ldots$ up to 15 . The box will hold just sixteen such counters, and, as it contains only fifteen, they can be moved about in the box relatively to one another. Initially they are put in the box in any order, but leaving the sixteenth

* There are two articles on the subject in the American Journal of Mathematics, 1879, vol. Ir, by Professors Woolsey Johnson and Storey; but the whole theory is deducible inmediately from the proposition I give in the text. 
cell or small square empty; the puzzle is to move them so that finally they occupy the position shown in the first of the annexed figures.
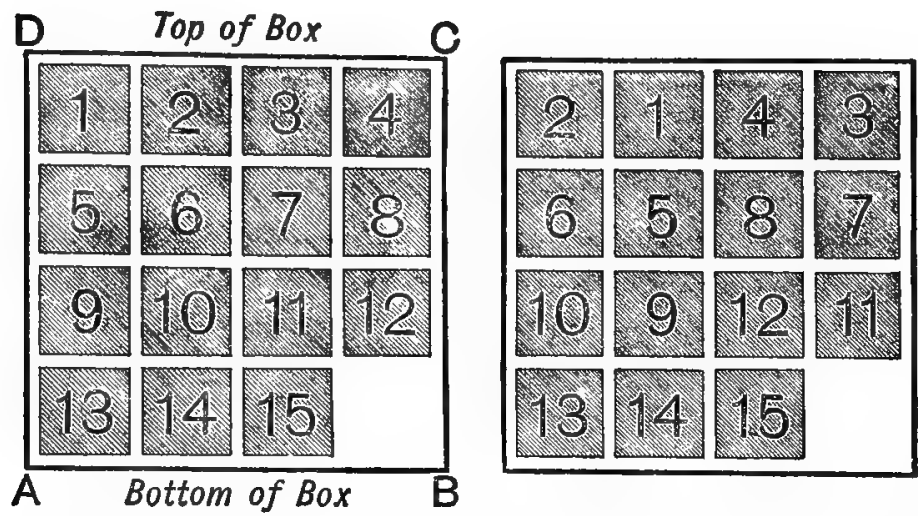

We may represent the various stages in the game by supposing that the blank space, occupying the sixteenth cell, is moved over the board, ending finally where it started.

The route pursued by the blank space may consist partly of tracks followed and again retraced, which have no effect on the arrangement, and partly of closed paths travelled round, which necessarily are cyclical permutations of an odd number of counters. No other motion is possible.

Now a cyclical permutation of $n$ letters is equivalent to $n-1$ simple interchanges; accordingly an odd cyclical permutation is equivalent to an even number of simple interchanges. Hence, if we move the counters so as to bring the blank space back into the sixteenth cell, the new order must differ from the initial order by an even number of simple interchanges. If therefore the order we want to get can be obtained from this initial order only by an odd number of interchanges, the problem is incapable of solution; if it can be obtained by an even number, the problem is possible.

Thus the order in the second of the diagrams given above is deducible from that in the first diagram by six interchanges; namely, by interchanging the counters 1 and 2,

B. $\mathbf{R}$ 
3 and 4,5 and 6, 7 and 8,9 and 10,11 and 12. Hence the one can be deduced from the other by moving the counters about in the box.

If however in the second diagram the order of the last three counters had been $13,15,14$, then it would have required seven interchanges of counters to bring them into the order given in the first diagram. Hence in this case the problem would be insoluble.

The easiest way of finding the number of simple interchanges necessary in order to obtain one given arrangement from another is to make the transformation by a series of cycles. For example, suppose that we take the counters in the box in any definite order, such as taking the successive rows from left to right, and suppose the original order and the final order to be respectively

$$
1,13,2,3,5,7,12,8,15,6,9,4,11,10,14,
$$

and $11,2,3,4,5,6,7,1,9,10,13,12,8,14,15$.

We can deduce the second order from the first by 12 simple interchanges. The simplest way of seeing this is to arrange the process in three separate cycles as follows :-

$$
\begin{array}{r|rr}
1,11,8 ; & 13,2,3,4,12,7,6,10,14,15,9 ; & 5 . \\
11,8,1 ; & 2,3,4,12,7,6,10,14,15,9,13 ; & 5 .
\end{array}
$$

Thus, if in the first row of figures 11 is substituted for 1 , then 8 for 11, then 1 for 8 , we have made a cyclical interchange of 3 numbers, which is equivalent to 2 simple interchanges (namely, interchanging 1 and 11, and then 1 and 8 ). Thus the whole process is equivalent to one cyclical interchange of 3 numbers, another of 11 numbers, and another of 1 number. Hence it is equivalent to $(2+10+0)$ simple interchanges. This is an even number, and thus one of these orders can be deduced from the other by moving the counters about in the box.

It is obvious that, if the initial order is the same as the required order except that the last three counters are in the order $15,14,13$, it would require one interchange to put them in the order 13,14, 15; hence the problem is insoluble.

If however the box is turned through a right angle, so as 
to make $A D$ the top, this rotation will be equivalent to 13 simple interchanges. For, if we keep the sixteenth square always blank, then such a rotation would change any order such as

$$
1,2,3,4,5,6,7,8,9,10,11,12,13,14,15,
$$

to $13,9,5,1,14,10,6,2,15,11,7,3,12,8,4$,

which is equivalent to $\mathbf{1 3}$ simple interchanges. Hence it will change the arrangement from one where a solution is impossible to one where it is possible, and vice versa.

Again, even if the initial order is one which makes a solution impossible, yet if the first cell and not the last is left blank it will be possible to arrange the fifteen counters in their natural order. For, if we represent the blank cell by $b$, this will be equivalent to changing the order

$$
1,2,3,4,5,6,7,8,9,10,11,12,13,14,15, b \text {, }
$$

to $b, 1,2,3,4,5,6,7,8,9,10,11,12,13,14,15$ :

this is a cyclical interchange of 16 things and therefore is equivalent to $\mathbf{1 5}$ simple interchanges. Hence it will change the arrangement from one where a solution is impossible to one where it is possible, and vice versa.

So, too, if it were permissible to turn the 6 and the 9 upside down, thus changing them to 9 and 6 respectively, this would be equivalent to one simple interchange, and therefore would change an arrangement where a solution is impossible to one where it is possible.

It is evident that the above principles are applicable equally to a rectangular box containing $m n$ cells or spaces and $m n-1$ counters which are numbered. Of course $m$ may be equal to $n$. If such a box is turned through a right angle, and $m$ and $n$ are both even, it will be equivalent to $m n-3$ simple interchangesand thus will change an impossible position to a possible one, and vice versa-but unless both $m$ and $n$ are even the rotation is equivalent to only an even number of interchanges. Similarly, if either $m$ or $n$ is even, and it is impossible to solve the problem when the last cell is left blank, then it will be possible to solve it by leaving the first cell blank. 
The problem may be made more difficult by limiting the possible movements by fixing bars inside the box which will prevent the movement of a counter transverse to their directions. We can conceive also of a similar cubical puzzle, but we could not work it practically except by sections.

THE TOWER of HaNoÏ. I may mention next the ingenious puzzle known as the Tower of Hanoü. It was brought out in 1883 by M. Claus (Lucas).

It consists of three pegs fastened to a stand, and of eight circular discs of wood or cardboard each of which has a hole in the middle through which a peg can be passed. These dises are of different radii, and initially they are placed all on one peg, so that the biggest is at the bottom, and the radii of the successive discs decrease as we ascend: thus the smallest disc is at the top. This arrangement is called the Tower. The problem is to shift the discs from one peg to another in such a way that a disc shall never rest on one smaller than itself, and finally to transfer the tower (i.e. all the discs in their proper order) from the peg on which they initially rested to one of the other pegs.

The method of effecting this is as follows. (i) If initially there are $n$ discs on the peg $A$, the first operation is to transfer gradually the top $n-1$ dises from the peg $A$ to the peg $B$, leaving the peg $C$ vacant: suppose that this requires $x$ separate transfers. (ii) Next, move the bottom disc to the peg $C$. (iii) Then, reversing the first process, transfer gradually the $n-1$ discs from $B$ to $C$, which will necessitate $x$ transfers. Hence; if it requires $x$ transfers of simple discs to move a tower of $n-1$ discs, then it will require $2 x+1$ separate transfers of single dises to move a tower of $n$ dises. Now with 2 dises it requires 3 transfers, i.e. $2^{2}-1$ transfers; hence with 3 discs the number of transfers required will be $2\left(2^{2}-1\right)+1$, that is, $2^{3}-1$. Proceeding in this way we see that with a tower of $n$ discs it will require $2^{n}-1$ transfers of single discs to effect the complete transfer. Thus the eight discs of the puzzle will require 255 single transfers. It will be noticed that every alternate move 
consists of a transfer of the smallest disc from one peg to another, the pegs being taken in cyclical order: further if the dises be numbered consecutively $1,2,3, \ldots$ beginning with the smallest, all those with odd numbers rotate in one direction, and all those with even numbers in the other direction.

Obviously, the discs may be replaced by cards numbered $1,2,3, \ldots n$; and if $n$ is not greater than 10 playing cards may be conveniently used.

De Parville gave an account of the origin of the toy which is a sufficiently pretty conceit to deserve repetition*. In the great temple at Benares, says he, beneath the dome which marks the centre of the world, rests a brass plate in which are fixed three diamond needles, each a cubit high and as thick as the body of a bee. On one of these needles, at the creation, God placed sixty-four discs of pure gold, the largest disc resting on the brass plate, and the others getting smaller and smaller up to the top one. This is the Tower of Bramah. Day and night unceasingly the priests transfer the discs from one diamond needle to another according to the fixed and immutable laws of Bramah, which require that the priest on duty must not move more than one disc at a time and that he must place this disc on a needle so that there is no smaller disc below it. When the sixty-four discs shall have been thus transferred from the needle on which at the creation God placed them to one of the other needles, tower, temple, and Brahmins alike will crumble into dust, and with a thunderclap the world will vanish.

The number of separate transfers of single discs which the Brahmins must make to effect the transfer of the tower is $2^{\text {st }}-1$, that is, is $18,446744,073709,551615$ : a number which, even if the priests never made a mistake, would require many thousands of millions of years to carry out.

Chinese Rinast. A somewhat more elaborate toy, known as Chinese Rings, which is on sale in most English toy-shops,

- La Nature, Paris, 1884, part I, pp. 285-286.

+ It was desoribed by Cardan in 1550 in his De Subtilitate, bk, $x \nabla$, paragraph 2, ed. Sponius, vol. Ir, p. 587; by Wallis in his Algebra, Latin edition, 1693, Opera, vol. II, oh\&p. cxi, pp. 472-478; and allusion is made to it also in Ozanam's Récréations, 1723 edition, vol. IV, p. 439. 
is represented in the accompanying figure. It consists of a number of rings hung upon a bar in such a manner that the ring at one end (say $A$ ) can be taken off or put on the bar at pleasure; but any other ring can be taken off or put on only when the one next to it towards $A$ is on, and all the rest towards $A$ are off the bar. The order of the rings cannot be changed.

Only one ring can be taken off or put on at a time. [In the toy, as usually sold, the first two rings form an exception to the rule. Both these can be taken off or put on together.

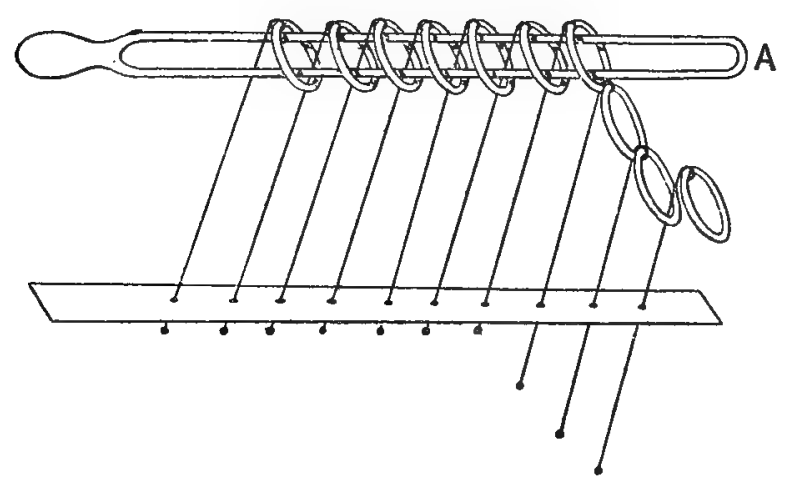

To simplify the discussion I shall assume at first that only one ring is taken off or put on at a time.] I proceed to show that, if there are $n$ rings, then in order to disconnect them from the bar, it will be necessary to take a ring off or to put a ring on either $\frac{1}{3}\left(2^{n+1}-1\right)$ times or $\frac{1}{3}\left(2^{n+1}-2\right)$ times according as $n$ is odd or even.

Let the taking a ring off the bar or putting a ring on the bar be called a step. It is usual to number the rings from the free end $A$. Let us suppose that we commence with the first $m$ rings off the bar and all the rest on the bar; and suppose that then it requires $x-1$ steps to take off the next ring, that is, it requires $x-1$ additional steps to arrange the rings so that the first $m+1$ of them are off the bar and all the rest are on it. Before taking these steps we can take off 
the $(m+2)$ th ring and thus it will require $x$ steps from our initial position to remove the $(m+1)$ th and $(m+2)$ th rings. Suppose that these $x$ steps have been made and that thus the first $m+2$ rings are off the bar and the rest on it, and let us find how many additional steps are now necessary to take off the $(m+3)$ th and $(m+4)$ th rings. To take these off we begin by taking off the $(m+4)$ th ring: this requires 1 step. Before we can take off the $(m+3)$ th ring we must arrange the rings so that the $(m+2)$ th ring is on and the first $m+1$ rings are off: to effect this, (i) we must get the $(m+1)$ th ring on and the first $m$ rings off, which requires $x-1$ steps, (ii) then we must put on the $(m+2)$ th ring, which requires 1 step, (iii) and lastly we must take the $(m+1)$ th ring off, which requires $x-1$ steps: these movements require in all $\{2(x-1)+1\}$ steps. Next we can take the $(m+3)$ th ring off, which requires 1 step; this leaves us with the first $m+1$ rings off, the $(m+2)$ th on, the $(m+3)$ th off and all the rest on. Finally to take off the $(m+2)$ th ring, (i) we get the $(m+1)$ th ring on and the first $m$ rings off, which requires $x-1$ steps, (ii) we take off the $(m+2)$ th ring, which requires 1 step, (iii) we take the $(m+1)$ th ring off, which requires $x-1$ steps: these movements require $\{2(x-1)+1\}$ steps.

Therefore, if when the first $m$ rings are off it requires $x$ steps to take off the $(m+1)$ th and $(m+2)$ th rings, then the number of additional steps required to take off the $(m+3)$ th and $(m+4)$ th rings is $1+\{2(x-1)+1\}+1+\{2(x-1)+1\}$, that is, is $4 x$.

To find the whole number of steps necessary to take off an odd number of rings we proceed as follows.

To take off the first ring requires 1 step;

$\therefore$ to take off the first 3 rings requires 4 additional steps;

$\therefore \quad$ " $\quad$ " ", $4^{2}$ "

In this way we see that the number of steps required to take off the first $2 n+1$ rings is $1+4+4^{2}+\ldots+4^{n}$, which is equal to $\frac{1}{3}\left(2^{2 n+2}-1\right)$.

To find the number of steps necessary to take off an even number of rings we proceed in a similar manner. 
To take off the first 2 rings requires 2 steps;

$\therefore$ to take off the first 4 , rings requires $2 \times 4$ additional steps;

$\therefore$ " " 6 " $\quad 2 \times 44^{2}$,

In this way we see that the number of steps required to take off the first $2 n$ rings is $2+(2 \times 4)+\left(2 \times 4^{2}\right)+\ldots+\left(2 \times 4^{n-1}\right)$, which is equal to $\frac{1}{3}\left(2^{2 n+1}-2\right)$.

If we take off or put on the first two rings in one step instead of two separate steps, these results become respectively $2^{2 n}$ and $2^{2 n-1}-1$.

I give the above analysis because it is the direct solution of a problem attacked unsuccessfully by Cardan in 1550 and by Wallis in 1693, and which at one time attracted some attention.

I proceed next to give another solution, more elegant though rather artificial. This, which is due to Monsieur Gros*, depends on a convention by which any position of the rings is denoted by a certain number expressed in the binary scale of notation in such a way that a step is indicated by the addition or subtraction of unity.

Let the rings be indicated by circles : if a ring is on the bar, it is represented by a circle drawn above the bar; if the ring is off the bar, it is represented by a circle below the bar. Thus figure $i$ below represents a set of seven rings of which the first two are off the bar, the next three are on it, the sixth is off it, and the seventh is on it.

Denote the rings which are on the bar by the digits 1 or 0 alternately, reckoning from left to right, and denote a ring which is off the bar by the digit assigned to that ring on the bar which is nearest to it on the left of it, or by a 0 if there is no ring to the left of it.

Thus the three positions indicated below are denoted respectively by the numbers written below them. The position represented in figure ii is obtained from that in figure $i$ by putting the first ring on to the bar, while the position represented in figure iii is obtained from that in figure $\mathrm{i}$ by taking the fourth ring off the bar.

* Théorie du Baguenodier, by L. Gros, Lyous, 1872. I take the account of this from Lucas, vol. i, part 7. 
It follows that every position of the rings is denoted by a number expressed in the binary scale: moreover, since in going from left to right every ring on the bar gives a variation (that is, 1 to 0 or 0 to 1 ) and every ring off the bar gives a continuation, the effect of a step by which a ring is taken off or put on the bar is either to subtract unity from this number or to add unity to it. For example, the number denoting the position of the rings in figure ii is obtained from the number denoting that un figure $i$ by adding unity to it. Similarly the number denoting the position of the rings in figure iii is obtained from the number denoting that in figure $\mathrm{i}$ by subtracting unity from it.

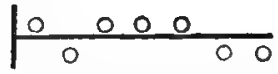

1101000

Figure i.

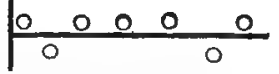

1101001

Figure ii.

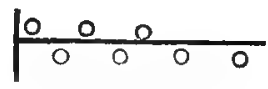

1100111

Figure iii.

The position when all the seven rings are off the bar is denoted by the number 0000000 : when all of them are on the bar by the number 1010101. Hence to change from one position to the other requires a number of steps equal to the difference between these two numbers in the binary scale. The first of these numbers is 0 : the second is equal to $2^{6}+2^{4}+2^{2}+1$, that is, to 85 . Therefore 85 steps are required. In a similar way we may show that to put on a set of $2 n+1$ rings requires $\left(1+2^{2}+\ldots+2^{2 n}\right)$ steps, that is, $\frac{1}{3}\left(2^{2 n+8}-1\right)$ steps ; and to put on a set of $2 n$ rings requires $\left(2+2^{3}+\ldots+2^{2 n-1}\right)$ steps, that is, $\frac{1}{8}\left(2^{2 n+1}-2\right)$ steps.

I append a table indicating the steps necessary to take off the first four rings from a set of five rings. The diagrams in the middle column show the successive position of the rings after each step. The number following each diagram indicates that position, each number being obtained from the one above it by the addition of unity. The steps which are bracketed together can be made in one movement, and, if thus effected, the whole process is completed in 7 movements instead of 10 steps: this is in accordance with the formula given above. 
Gros asserted that it is possible to take from 64 to 80 steps a minute, which in my experience is a rather high estimate. If we accept the lower of these numbers, it would be possible to take off 10 rings in less than 8 minutes; to take off 25 rings would require more than 582 days, each of ten hours work; and to take off 60 rings would necessitate no less than $768614,336404,564650$ steps, and would require nearly 55000,000000 years work-assuming of course that no mistakes were made.

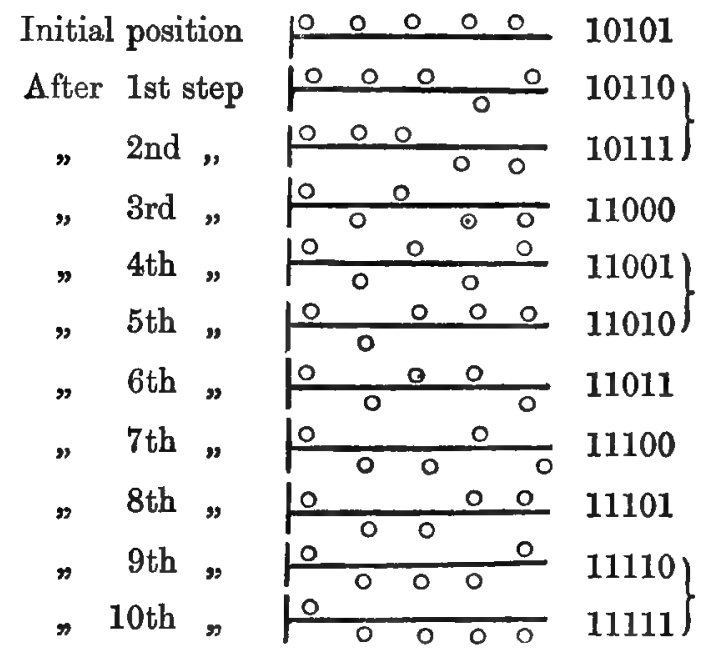

Problems connected with a Pack of Cards. An ordinary pack of playing cards can be used to illustrate many questions depending on simple properties of numbers, or involving the relative position of the cards. In problems of this kind, the principle of solution generally consists in re-arranging the pack in a particular manner so as to bring the card into some definite position. Any such re-arrangement is a species of shuffling.

I shall treat in succession of problems connected with Shuffing a Pack, Arrangements by Rows and Columns, the Determination of a Pair out of $\frac{1}{2} n(n+1)$ Pairs, Gergonne's Pile Problem, and the game known as the Mouse Trap. 
Shuffuting a PACK. Any system of shuffing a pack of cards, if carried out consistently, leads to an arrangement which can be calculated; but tricks that depend on it generally require considerable technical skill.

Suppose for instance that a pack of $n$ cards is shuffled, as is not unusual, by placing the second card on the first, the third below these, the fourth above them, and so on. The theory of this system of shuffling is due to Monge*. The following are some of the results and are not difficult to prove directly.

One shuffle of a pack of $2 p$ cards will move the card which was in the $x_{0}$ th place to the $x_{1}$ th place, where $x_{1}=\frac{1}{2}\left(2 p+x_{0}+1\right)$ if $x_{0}$ is odd, and $x_{1}=\frac{1}{2}\left(2 p-x_{0}+2\right)$ if $x_{0}$ is even. For instance, if a complete pack of 52 cards is shuffled as described above, the 18th card will remain the 18 th card. If an écarté pack of 32 cards is so shuffled, the 7 th and the 20 th cards will change places.

By repeated applications of the above formulae we can show that the effect of $m$ such shuffles is to move the card which was initially in the $x_{0}$ th place to the $x_{m}$ th place, where

$$
2^{m+1} x_{m}=(4 p+1)\left(2^{m-1} \pm 2^{m-2} \pm \ldots \pm 2 \pm 1\right) \pm 2 x_{0}+2^{m} \pm 1,
$$

the sign \pm representing an ambiguity of sign.

Again, in any pack of $n$ cards after a certain number of shufflings, not greater than $n$, the cards will return to their primitive order. This will always be the case as soon as the original top card occupies that position again. To determine the number of shuffles required for a pack of $2 p$ cards, it is sufficient to put $x_{m}=x_{0}$ and find the smallest value of $m$ which satisfies the resulting equation for all values of $x_{0}$ from 1 to $2 p$.

The result can however be obtained more easily if the cards

* Monge's investigations are printed in the Ménoires de l'Académie des Sciences, Paris, 1773, pp. 390-412. Among those who have studied the subject afresh I may in particular mention V. Bouniakowski, Bulletin physico-mathematique de St Pétersbourg, 1857, vol. xv, pp. 202-205, summarised in the Nouvelles annales de mathématiques, 1858, Bulletin, pp. 66-67; T. de St Laurent, Mémoires de l'Académie de Gard, 1865; L. Tanner, Educational Times Reprints, 1880, vol. xxxIII, pp. 73-75; M. J. Bourget, Liouville's Journal, 1882, pp. 413-431; H. F. Baker, Transactions of the British Association for 1910, pp. 526-528; and P. H. Cowell, The Field, 2 April, 1921, p. 444. 
are numbered from the bottom of the original pack. Doing this, we can show that if after $s$ shuffles a card is in the $r$ th place from the bottom, its original number from the bottom was the difference between $2^{s} \times r$ and the nearest multiple of $4 p+1$. Hence, if $m$ shuffles are required to restore the original order, $m$ is the least number for which $2^{m}+1$ or $2^{m}-1$ is divisible by $4 p+1$. The number for a pack of $2 p+1$ cards is the same as that for a pack of $2 p$ cards. With an écarté pack of 32 cards, six shuffles are sufficient; with a pack of $2^{n}$ cards, $n+1$ shuffles are sufficient; with a full pack of 52 cards, twelve shuffles are sufficient; with a pack of 13 cards ten shuffles are sufficient; while with a pack of 50 cards fifty shuffles are required; and so on.

W. H. H. Hudson* has also shown that, whatever is the law of shuffling, yet if it is repeated again and again on a pack of $n$ cards, the cards will ultimately fall into their initial positions after a number of shufflings not greater than the greatest possible L. C.M. of all numbers whose sum is $n$.

For suppose that any particular position is occupied after the 1st, 2 nd, ..., pth shuffles by the cards $A_{1}, A_{2}, \ldots, A_{p}$ respectively, and that initially the position is occupied by the card $A_{0}$. Suppose further that after the $p$ th shuffle $A_{0}$ returns to its initial position, therefore $A_{0}=A_{p}$. Then at the second shuffling $A_{2}$ succeeds $A_{1}$ by the same law by which $A_{1}$ succeeded $A_{0}$ at the first; hence it follows that previous to the second shuffing $A_{2}$ must have been in the place occupied by $A_{1}$ previous to the first. Thus the cards which after the successive shuffles take the place initially occupied by $A_{1}$ are $A_{2}, A_{3}, \ldots$, $A_{p}, A_{1}$; that is, after the $p$ th shuffle $A_{1}$ has returned to the place initially occupied by it: and so for all the other cards $A_{2}, A_{\mathrm{s}}, \ldots, A_{p-1}$.

Hence the cards $A_{1}, A_{2}, \ldots, A_{p}$ form a cycle of $p$ cards, one or other of which is always in one or other of $p$ positions in the pack, and which go through all their changes in $p$ shuffings. Let the number $n$ of the pack be divided into $p, q, r, \ldots$ such cycles, whose sum is $n$; then the L.C.M. of $p, q, r, \ldots$ is the

- Educational Times Reprints, London, 1865, vol. u, p. 105. 
utmost number of shufflings necessary before all the cards will be brought back to their original places. In the case of a pack of 52 cards, the greatest L.C.M. of numbers whose sum is 52 is 180180 .

Arrangements by Rows and Columns. A not uncommon trick, which rests on a species of shuffling, depends on the obvious fact that if $n^{3}$ cards are arranged in the form of a square of $n$ rows, each containing $n$ cards, then any card will be defined if the row and the column in which it lies are mentioned.

This information is generally elicited by first asking in which row the selected card lies, and noting the extreme lefthand card of that row. The cards in each column are then taken up, face upwards, one at a time beginning with the lowest card of each column and taking the columns in their order from right to left-each card taken up being placed on the top of those previously taken up. The cards are then dealt out again in rows, from left to right, beginning with the top left-hand corner, and a question is put as to which row contains the card. The selected card will be that card in the row mentioned which is in the same vertical column as the card which was originally noted.

The trick is improved by allowing the pack to be cut as often as is liked before the cards are re-dealt, and then giving one cut at the end so as to make the top card in the pack one of those originally in the top row. For instance, take the

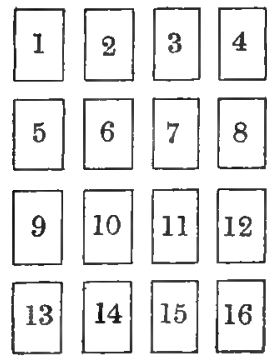

Figure i.

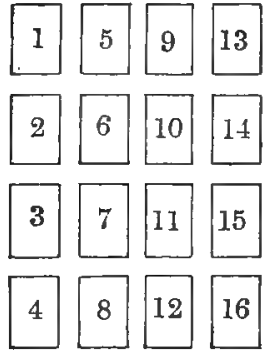

Figure ii. 
case of 16 cards. The first and second arrangements may be represented by figures $i$ and ii. Suppose we are told that in figure $i$ the card is in the third row, it must be either 9,10 , 11, 12: hence, if we know in which row of figure ii it lies, it is determined. If we allow the pack to be cut between the deals, we must secure somehow that the top card is either $1,2,3$, or 4, since that will leave the cards in each row of figure ii unaltered though the positions of the rows will be changed.

Determination of a Selected pair of CaRDs out of $\frac{1}{2} n(n+1)$ GIVEN PAIRS*. Another common trick is to throw twenty cards on to a table in ten couples, and ask someone to select one couple. The cards are then taken up, and dealt out in a certain manner into four rows each containing five cards. If the rows which contain the given cards are indicated, the cards selected are known at once.

This depends on the fact that the number of homogeneous products of two dimensions which can be formed out of four things is 10. Hence the homogeneous products of two dimensions formed out of four things can be used to define ten things

Suppose that ten pairs of cards are placed on a table and someone selects one couple. Take up the cards in their

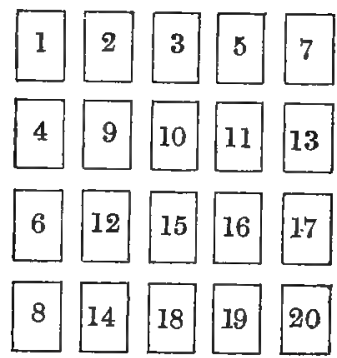

couples. Then the first two cards form the first couple, the next two the second couple, and so on. Deal them out in four rows each containing five cards according to the scheme shown above.

* Bachet, problem xvII, avertissement, p. 146 et seq. 
The first couple ( 1 and 2 ) are in the first row. Of the next couple (3 and 4), put one in the first row and one in the second. Of the next couple ( 5 and 6 ), put one in the first row and one in the third, and so on, as indicated in the diagram. After filling up the first row proceed similarly with the second row, and so on.

Enquire in which rows the two selected cards appear. If only one line, the $m \mathrm{th}$, is mentioned as containing the cards, then the required pair of cards are the $m$ th and $(m+1)$ th cards in that line. These occupy the clue squares of that line. Next, if two lines are mentioned, then proceed as follows. Let the two lines be the $p$ th and the $q$ th and suppose $q>p$. Then that one of the required cards which is in the qth line will be the $(q-p)$ th card which is below the first of the clue squares in the $p$ th line. The other of the required cards is in the $p$ th line and is the $(q-p)$ th card to the right of the second of the clue squares.

Bachet's rule, in the form in which I have given it, is applicable to a pack of $n(n+1)$ cards divided into couples, and dealt in $n$ rows each containing $n+1$ cards; for there are $\frac{1}{2} n(n+1)$ such couples, also there are $\frac{1}{2} n(n+1)$ homogeneous products of two dimensions which can be formed out of $n$ things. Bachet gave the diagrams for the cases of 20,30 , and 42 cards : these the reader will have no difficulty in constructing for himself, and I have enunciated the rule for 20 cards in a form which covers all the cases.

I have seen the same trick performed by means of a sentence and not by numbers. If we take the case of ten couples, then after collecting the pairs the cards must be dealt in four rows each containing five cards, in the order indicated by the sentence Matas dedit nomen Cocis. This sentence must be imagined as written on the table, each word forming one line. The first card is dealt on the $M$. The next card (which is the pair of the first) is placed on the second $m$ in the sentence, that is, third in the third row. The third card is placed on the a. The fourth card (which is the pair of the third) is placed on the second $a$, that is, fourth in the first row. Each 
of the next two cards is placed on a $t$, and so on. Enquire in which rows the two selected cards appear. If two rows are mentioned, the two cards are on the letters common to the words that make these rows. If only one row is mentioned, the cards are on the two letters common to that row.

The reason is obvious: let us denote each of the first pair by an $a$, and similarly each of any of the other pairs by an $e, i, o, c, d, m, n, s$, or $t$ respectively. Now the sentence Matas dedit nomen Cocis contains four words each of five letters; ten letters are used, and each letter is repeated only twice. Hence, if two of the words are mentioned, they will have one letter in common, or, if one word is mentioned, it will have two like letters.

To perform the same trick with any other number of cards we should require a different sentence.

The number of homogeneous products of three dimensions which can be formed out of four things is 20 , and of these the number consisting of products in which three things are alike and those in which three things are different is 8 . This leads to a trick with 8 trios of things, which is similar to that last given-the cards being arranged in the order indicated by the sentence Lanata levete livini novoto.

I believe that these arrangements by sentences are wellknown, but I am not aware who invented them.

Gergonne's Pile Problem. Before discussing Gergonne's theorem I will describe the familiar three pile problem, the theory of which is included in his results.

The Three Pile Problem*. This trick is usually performed as follows. Take 27 cards and deal them into three piles, face upwards. By "dealing" is to be understood that the top card is placed as the bottom card of the first pile, the second card in the pack as the bottom card of the second pile, the third card as the bottom card of the third pile, the fourth card on the top of the first one, and so on: moreover I assume that throughout

* The trick is mentioned by Bachet, problem xviI, p. 143, but his analysis of it is insufficient. 
the problem the cards are held in the hand face upwards. The result can be modified to cover any other way of dealing.

Request a spectator to note a card, and remember in which pile it is. After finishing the deal, ask in which pile the card is. Take up the three piles, placing that pile between the other two. Deal again as before, and repeat the question as to which pile contains the given card. Take up the three piles again, placing the pile which now contains the selected card between the other two. Deal again as before, but in dealing note the middle card of each pile. Ask again for the third time in which pile the card lies, and you will know that the card was the one which you noted as being the middle card of that pile. The trick can be finished then in any way that you like. The usual method-but a very clumsy one--is to take up the three piles once more, placing the named pile between the other two as before, when the selected card will be the middle one in the pack, that is, if 27 cards are used it will be the 14th card.

The trick is often performed with 15 cards or with 21 cards, in either of which cases the same rule holds.

Gergonne's Generalization. The general theory for a pack of $m^{m}$ cards was given by M. Gergonne*. Suppose the pack is arranged in $m$ piles, each containing $m^{m-1}$ cards, and that, after the first deal, the pile indicated as containing the selected card is taken up ath; after the second deal, is taken up bth; and so on, and finally after the $m$ th deal, the pile containing the card is taken up $k$ th. Then when the cards are collected after the $m$ th deal the selected card will be $n$th from the top where

if $m$ is even, $\quad n=k m^{m-1}-j m^{m-9}+\ldots+b m-a+1$,

if $m$ is odd, $\quad n=k m^{m-1}-j m^{m-2}+\ldots-b m+a$.

For example, if a pack of 256 cards (i.e. $m=4$ ) was given, and anyone selected a card out of it, the card could be determined by making four successive deals into four piles of 64 cards each, and after each deal asking in which pile the

* Gergonne's Annales de Mathématiques, Nismes, 1813-4, vol. I7, pp. 276283.

B. $\mathbb{R}$ 
selected card lay. The reason is that after the first deal you know it is one of sixty-four cards. In the next deal these sixty-four cards are distributed equally over the four piles, and therefore, if you know in which pile it is, you will know that it is one of sixteen cards. After the third deal you know it is one of four cards. After the fourth deal you know which card it is.

Moreover, if the pack of 256 cards is used, it is immaterial in what order the pile containing the selected card is taken up after a deal. For, if after the first deal it is taken up ath, after the second $b$ th, after the third $c$ th, and after the fourth $d$ th, the card will be the $(64 d-16 c+4 b-a+1)$ th from the top of the pack, and thus will be known. We need not take up the cards after the fourth deal, for the same argument will show that it is the $(64-16 c+4 b-a+1)$ th in the pile then indicated as containing it. Thus if $a=3, b=4, c=1, d=2$, it will be the 62 nd card in the pile indicated after the fourth deal as containing it and will be the 126 th card in the pack as then collected.

In exactly the same way a pack of twenty-seven cards may be used, and three successive deals, each into three piles of nine cards, will suffice to determine the card. If after the deals the pile indicated as containing the given card is taken up ath, $b$ th, and cth respectively, then the card will be the $(9 c-3 b+a)$ th in the pack or will be the $(9-3 b+a)$ th card in the pile indicated after the third deal as containing it.

The method of proof will be illustrated sufficiently by considering the usual case of a pack of twenty-seven cards, for which $m=3$, which are dealt into three piles each of nine cards.

Suppose that, after the first deal, the pile containing the selected card is taken up ath: then (i) at the top of the pack there are $a-1$ piles each containing nine cards; (ii) next there are 9 cards, of which one is the selected card; and (iii) lastly there are the remaining cards of the pack. The cards are dealt out now for the second time: in each pile the bottom $3(a-1)$ cards will be taken from (i), the next 3 cards from (ii), and the remaining $9-3 a$ cards from (iii). 
Suppose that the pile now indicated as containing the selected card is taken up bth: then (i) at the top of the pack are $9(b-1)$ cards; (ii) next are $9-3 a$ cards; (iii) next are 3 cards, of which one is the selected card; and (iv) lastly are the remaining cards of the pack. The cards are dealt out now for the third time : in each pile the bottom $3(b-1)$ cards will be taken from (i), the next $3-a$ cards will be taken from (ii), the next card will be one of the three cards in (iii), and the remaining $8-3 b+a$ cards are from (iv).

Hence, after this deal, as soon as the pile is indicated, it is known that the card is the $(9-3 b+a)$ th from the top of that pile. If the process is continued by taking up this pile as $c$ th, then the selected card will come out in the place $9(c-1)+(8-3 b+a)+1$ from the top, that is, will come out as the $(9 c-3 b+a)$ th card.

Since, after the third deal, the position of the card in the pile then indicated is known, it is easy to notice the card, in which case the trick can be finished in some way more effective than dealing again.

If we put the pile indicated always in the middle of the pack we have $a=2, b=2, c=2$, hence $n=9 c-3 b+a=14$, which is the form in which the trick is usually presented, as was explained above on page 241.

I have shown that if $a, b, c$ are known, then $n$ is determined. We may modify the rule so as to make the selected card come out in any assigned position, say the $n$ th. In this case we have to find values of $a, b, c$ which will satisfy the equation $n=9 c-3 b+a$, where $a, b, c$ can have only the values 1,2 , or 3 .

Hence, if we divide $n$ by 3 and the remainder is 1 or 2 , this remainder will be $a$; but, if the remainder is 0 , we must decrease the quotient by unity so that the remainder is 3 , and this remainder will be $a$. In other words $a$ is the smallest positive number (exclusive of zero) which must be subtracted from $n$ to make the difference a multiple of 3 .

Next let $p$ be this multiple, i.e. $p$ is the next lowest integer to $n / 3$ : then $3 p=9 c-3 b$, therefore $p=3 c-b$. Hence $b$ is 
the smallest positive number (exclusive of zero) which must be added to $p$ to make the sum a multiple of 3 , and $c$ is that multiple.

A couple of illustrations will make this clear. Suppose we wish the card to come out 22 nd from the top, therefore $22=9 c-3 b+a$. The smallest number which must be subtracted from 22 to leave a multiple of 3 is 1 , therefore $a=1$. Hence $22=9 c-3 b+1$, therefore $7=3 c-b$. The smallest number which must be added to 7 to make a multiple of 3 is 2 , therefore $b=2$. Hence $7=3 c-2$, therefore $c=3$. Thus $a=1$, $b=2, c=3$.

Again, suppose the card is to come out 21st. Hence $21=9 c-3 b+a$. Therefore $a$ is the smallest number which subtracted from 21 makes a multiple of 3 , therefore $a=3$. Hence $6=3 c-b$. Therefore $b$ is the smallest number which added to 6 makes a multiple of 3 , therefore $b=3$. Hence $9=3 c$, therefore $c=3$. Thus $a=3, b=3, c=3$.

If any difficulty is experienced in this work, we can proceed thus. Let $a=x+1, b=3-y, c=z+1$; then $x, y, z$ may have only the values 0,1 , or 2 . In this case Gergonne's equation takes the form $9 z+3 y+x=n-1$. Hence, if $n-1$ is expressed in the ternary scale of notation, $x, y, z$ will be determined, and therefore $a, b, c$ will be known.

The rule in the case of a pack of $m^{m}$ cards is exactly similar. We want to make the card come out in a given place. Hence, in Gergonne's formula, we are given $n$ and we have to find $a, b, \ldots, k$. We can effect this by dividing $n$ continually by $m$, with the convention that the remainders are to be alternately positive and negative and that their numerical values are to be not greater than $m$ or less than unity.

An analogous theorem with a pack of $l m$ cards can be constructed. C. T. Hudson and L. E. Dickson* have discussed the general case where such a pack is dealt $n$ times, each time into $l$ piles of $m$ cards; and they have shown how the piles must be

- Educational Times Reprints, 1868, vol. Ix, pp. 89-91; and Bulletin of the Amerioan Mathematical Society, New York, April, 1895, vol. I, pp. 184188. 
taken up in order that after the $n$th deal the selected card may be $r$ th from the top.

The principle will be sufficiently illustrated by one example treated in a manner analogous to the cases already discussed. For instance, suppose that an écarté pack of 32 cards is dealt into four piles each of 8 cards, and that the pile which contains some selected card is picked up ath. Suppose that on dealing again into four piles, one pile is indicated as containing the selected card, the selected card cannot be one of the bottom $2(a-1)$ cards, or of the top $8-2 a$ cards, but must be one of the intermediate 2 cards, and the trick can be finished in any way, as for instance by the common conjuring ambiguity of asking someone to choose one of them, leaving it doubtful whether the one he takes is to be rejected or retained.

The Mouse Trap. Treize. I will conclude this chapter with the bare mention of another game of cards, known as the Mouse Trap, the discussion of which involves some rather difficult algebraic analysis.

It is played as follows. A set of cards, marked with the numbers $1,2,3, \ldots, n$, is dealt in any order, face upwards, in the form of a circle. The player begins at any card and counts round the circle always in the same direction. If the kth card has the number $k$ on it-which event is called a hit-the player takes up the card and begins counting afresh. According to Cayley, the player wins if he thus takes up all the cards, and the cards win if at any time the player counts up to $n$ without being able to take up a card.

For example, if a pack of only four cards is used and these cards come in the order 3214, then the player would obtain the second card 2 as a hit, next he would obtain 1 as a hit, but if he went on for ever he would not obtain another hit. On the other hand, if the cards in the pack were initially in the order 1423, the player would obtain successively all four cards in the order $1,2,3,4$.

The problem may be stated as the determination of what hits and how many hits can be made with a given number of 
cards; and what permutations will give a certain number of hits in a certain order.

Cayley* showed that there are 9 arrangements of a pack of four cards in which no hit will be made, 7 arrangements in which only one hit will be made, 3 arrangements in which only two hits will be made, and 5 arrangements in which four hits will be made.

Prof. Steen $\uparrow$ has investigated the general theory for a pack of $n$ cards. He has shown how to determine the number of arrangements in which $x$ is the first hit [Arts. 3-5]; the number of arrangements in which 1 is the first hit and $x$ is the second hit [Art. 6]; and the number of arrangements in which - 2 is the first hit and $x$ the second hit [Arts. 7-8]; but beyond this point the theory has not been carried. It is obvious that, if there are $n-1$ hits, the $n$th hit will necessarily follow.

The French game of treize is very similar. It is played with a full pack of fifty-two cards (knave, queen, and king counting as 11, 12, and 13 respectively). The dealer calls out $1,2,3, \ldots, 13$, as he deals the 1 st, 2 nd, 3rd, ..., 13th cards respectively. At the beginning of a deal the dealer offers to lay or take certain odds that he will make a hit in the thirteen cards next dealt.

* Quarterly Journal of Mathematics, 1878, vol. xv, pp. 8-10.

+ Ibid., vol. xv, pp. 230-241. 


\section{CHAPTER XII.}

\section{MISCELLANEOUS PROBLEMS (continued).}

This book was stereotyped some years ago, and I have explained above that subsequent additions have had to be fitted in where I could best find a place for them. In this chapter I put together a few additional problems for which I have been unable to find room in the chapters in which they would naturally have been included. Preferably the contents of this and the last chapters should have been inserted in the first four chapters, but to avoid such extensive rearrangement of the matter, I print these problems here.

I begin with Arithmetical Recreations, somewhat different in character to the selected typical examples given in chapter I, taking in succession Digit Questions, Restorations, and Calendar Problems.

Digit QUestions. Certain questions about digits of numbers are, by custom, presented under the title Arithmetical Recreations. Legendre gave a few examples of the kind.

Here are two time-honoured and easy instances, interesting because of their ancient lineage. The first of them is as follows: If $n$ digits are required in the pagination of a book, how many pages are contained therein; for instance, $n=3001$ ?

The analysis is obvious. The first 999 pages require the use of $(9+180+2700)$ digits. But 112 additional digits are employed, and these suffice to identify 28 more pages. Therefore the total number of pages is $999+28$, that is, 1027 .

Here is another example on elementary arithmetic. The numbers from 1 upwards are written consecutively. What is the $n$th digit: for instance, $n=500,000$ ?

The numbers from 1 to 99,999 inclusive require 488,889 digits. Hence we want the 11,111th digit in the series of six-digit 
numbers starting with 100,000 . We have $11,111=6 \times 1851+5$. Hence we want the 5 th digit in 101,851 , and this is 5 .

Arithmetical Restorations. I take next a class of problems dealing with the reconstruction of arithmetical sums from which various digits have been erased. Some of these questions are easy, some difficult. This kind of exercise has attracted a good deal of attention in recent years. I give examples of three kinds of restoration.

Class $A$. The solutions of one group of these restoration questions depend on the well-known propositions that every number

$$
a+10 b+10^{2} c+10^{3} d+\ldots
$$

is equal to any of certain expressions such as,

$$
\begin{aligned}
& M(9)+a+b+c+d+\ldots \\
& M(11)+a-b+c-d+\ldots \\
& M(33)+(a+10 b)+(c+10 d)+(e+10 f)+\ldots \\
& M(101)+(a+10 b)-(c+10 d)+(e+10 f)-\ldots \\
& M(n)+\left(a+10 b+10^{2} c\right)+\left(d+10 e+10^{2} f\right)+\ldots \\
& M(n)+\left(a+10 b+10^{2} c\right)-\left(d+10 e+10^{2} f\right)+\ldots
\end{aligned}
$$

where, in the penultimate line, $m=27$, or 37 , or 111 , and in the last line, $n=7$, or 13 , or 77 , or 91 , or 143 .

Examples, depending on such propositions, are not uncommon. Here are four easy instances of this class of questions.

(i) The product of 417 and $.1 \ldots$ is $9 \ldots 057$. Find the missing digits, each of which is represented by a dot. If the digits in the undetermined multiplier are denoted in order by $a, b, c, d$, and we take the steps of the multiplication in their reverse order, we obtain successively $d=1, c=2, b=9$. Also the product has 7 digits, therefore $a=2$. Hence the product is $9,1 \$ 1,057$.

(ii) The seven-digit number 70..34. is exactly divisible by 792. Find the missing digits, each of which is represented by a dot. Since 792 is $8 \times 9 \times 11$ we can easily show that the number is $7,054,344$.

(iii) The five-digit number 4.18 , is divisible by 101. Find the missing digits*.

* P. Delens, Problèmes d'Arithmétique Amusante, Paris, 1914, p. 55. 
Denote the two missing digits, from right to left, by $x$ and $y$. Applying the theorem for 101, noting that each of the unknowns cannot exceed 9 , and for convenience putting $y=10-z$, this equation gives $z=1, x=7, y=9$. Hence the number is 49,187 .

(iv) The four-digit number $.8 \ldots$ is divisible by 1287 . Find the missing digits*.

Denote these digits, from right to left, by $x, y, z$. We have $1287=9 \times 11 \times 13$. Applying the suitable propositions, and noting that each of the unknowns cannot exceed 9 , we get $x=1$, $y=6, z=3$. Hence the number is 3861 .

(v) As a slightly harder example of this type suppose we know that $6.80 .8 \ldots 51$ is exactly divisible by 73 and 137 . Find the missing digits $\dagger$. The data suffice to determine the number, which is $6,780,187,951$.

Class $B$. Another and more difficult class of restoration problems is illustrated by the following examples. Their solutions involve analytical skill which cannot be reduced to rules.

(i) I begin with an easy instance, said to be of Hindoo origin, in which the problem is to restore the missing digits in the annexed division sum where a certain six-digit number when divided by a three-digit number gives a three-digit resultt.

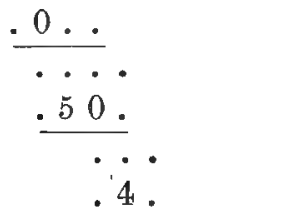

The solution involves no difficulty. The answer is that the divisor is 215 , and the quotient 573 ; the solution is unique.

(ii) As a more difficult specimen I give the following problem, proposed in 1921 by Prof. Schub of Delft. A certain sevendigit integer when divided by a certain six-digit integer gives a result whose integral part is a two-digit number and whose fractional part is a ten-digit expression of which the last nine

* P. Delens, Problèmes d'Arithmétique Amusante, Paris, 1914, p. 57.

$\dagger$ Ibid. p. 60.

$\ddagger$ American Mathematical Monthly, 1921, vol. zxviii, p. 37. 
digits form a repeating decimal, as indicated in the following work, where a bar has been put above the repeating digits. It is required to restore the working*. This problem is remarkable from the fact that not a single digit is given explicitly.

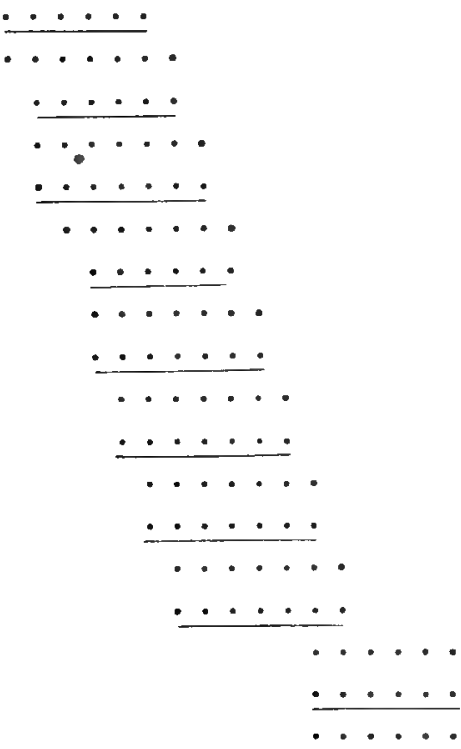

The answer is that the divisor is 667334 and the dividend is 7752341.

Here are three additional examples of arithmetical restorations $\dagger$. The solutions are lengthy and involve much empirical work.

(iii) The first of these Berwick questions is as follows. In the following division sum all the digits, except the seven "7's" shown, have been erased: each missing digit may be $1,2,3,4$, $5,6,7,8,9$, or (except in the first digit of a line) 0 . Observe

* American Mathematical Monthly, 1921, vol. xxviii, p. 61.

+ All are due to W. E. H. Berwick. The "7" problem appeared in the School World, July and October 1906, vol. viii, pp. 280, 320; the " 4 " problem appeared in the Mathematical Gazette, 1920, vol. x, pp. 43, 359-360; the " 5 " problem in the same paper, vol. $x$, p. 361, vol. $x i$, p. 8 . 
that every step in the working consists of two lines each of which contains an equal number of digits. The problem is to restore the whole working of the sum. The solution is unique and gives a divisor of 125473 and a quotient of 58781 .

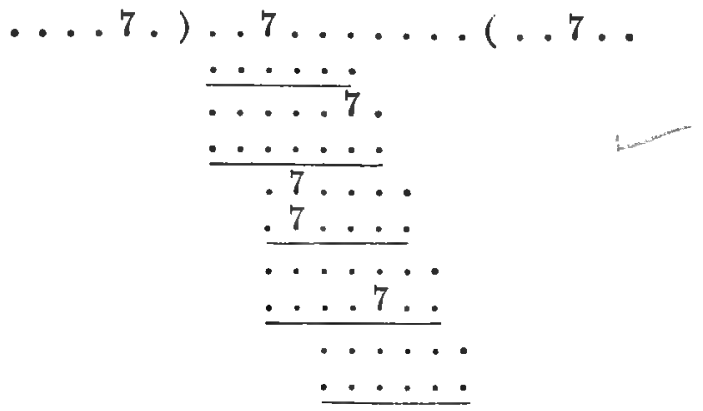

(iv) The second problem is similar and requires the restoration of the digits in the following division sum, where the position of four " 4 's" is given,

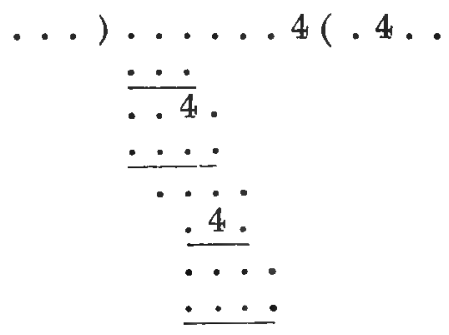

To this problem there are four solutions, the divisors being 846 , $848,943,949$; and the respective quotients $1419,1418,1418$, 1416.

If we propound the problem (using five "4's") thus:

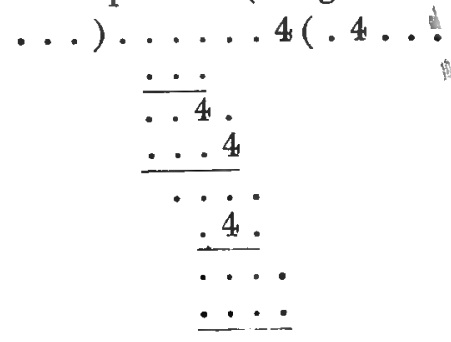


there is only one solution, and some will think this is a better form in which to enunciate it.

(v) In the last of these Berwick examples, it is required to restore the working of the following division sum where all the digits, except five "5's," have been erased.

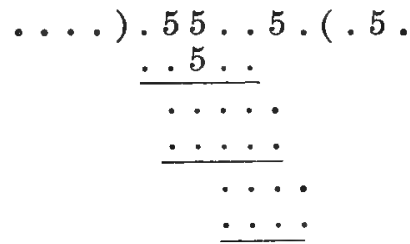

To this problem there is only one solution, the divisor being 3926 and the quotient 652 .

Class C. A third class of digit problems depends on finding the values of certain symbols which represent specified numbers. Two examples will suffice.

(i) Here is a very simple illustrative specimen. The result of multiplying $b c$ by $b c$ is $a b c$, where the letters stand for certain numbers. What are the numbers? A brief examination shows that $b c$ stands for 25, and therefore $a$ stands for 6 .

(ii) Here is another example. The object is to find the digits represented by letters in the following sum*:

$$
\begin{gathered}
a b) c d e e b(b f b \\
\frac{c e b}{g g e} \\
\frac{g c h}{c e b} \\
c e b
\end{gathered}
$$

A solution may be obtained thus: Since the product of $b$ by $b$ is a number which ends in $b, b$ must be 1,5 , or 6 . Since the product of $a b$ by $b$ is a number of three digits, $b$ cannot be 1 . The result of the subtraction of $h$ from $e$ is $e$, hence $h=0$, and therefore if $b=5$ we have $f$ even, and if $b=6$ we have $f=5$. Also the result of the subtraction of $c$ from $g$ is $c$, hence $g=2 c$, and therefore $c$ cannot be greater than 4 : from which it follows 
that $b$ cannot be 6 . A few trials now show that the question arose from the division of 19,775 by 35 .

It is possible to frame digit restoration examples of a mixed character involving the difficulties of all the examples given above, and to increase the difficulty by expressing them in a non-denary scale of notation. But such elaborations do not add to the interest of the questions.

Calendar Problems. The formulae given by Gauss and Zeller, which I quoted in former editions of this work, serve to solve all questions likely to occur about dates, days of the week, Easter, etc. Here are two easy but elegant questions on the Gregorian Calendar of a somewhat different nature.

The first is as follows: it is due I believe to E. Fourrey. In the century and a half between 1725 and 1875 the French fought and won a certain battle on 22 April of one year, and 4382 days later, also on 22 April, they gained another victory. The sum of the digits of the years is $4 \Theta$. Find the dates of the battles.

To solve it we notice that $4382=12 \times 365+2$. Hence the date of the second battle was 12 years after that of the first battle; but only two leap years had intervened, and therefore the year 1800 must be within the limiting dates. Thus 1788 and 1800,1789 and $1801, \ldots \ldots 1800$ and 1812, are the only possible years. Of the years thus suggested 1796 and 1808 alone give 40 as the sum of their digits. Hence the battles were fought on the 22 April, 1796 (Mondovi under Napoleon) and 22 April, 1808 (Eckmuhl under Davoust).

The other of these questions is to show that the first or last day of every alternate century must be a Monday. This follows from knowing any one assigned date, and the fact that the Gregorian cycle is completed in 400 years.

I proceed next to mention as additional Geometrical Recreations, the Five Disc Problem, Tesselation with Super-Dominoes, Geometrical Dissections, the Window-Reader Problem, and Compass Problems.

The Five Disc Problem. The problem of completely covering a fixed red circular space by placing over it, one at a 
time, five smaller equal circular tin discs is familiar to frequenters of English fairs. Its effectiveness depends on making the tin dises as small as possible, and therefore leads to the interesting geometrical question of finding the size of the smallest tin discs which can be used for the purpose.

The problem is soluble if the radius of each tin dise is just greater than three-fifths of the radius of the red circle. Of course in a show, the visitor is not allowed to move the discs when once he has put them down, and it is only rarely, very rarely, that he succeeds empirically in placing them correctly.

The rule works out thus. If $O$ is the centre of the red circle, $a$ its radius, and $A O B$ a diameter of it, we take on $O A$ a point $P$ such that approximately $O P=a / 40$. We then place the first tin dise with its centre on $O B$, and so that $P$ is on its edge: suppose that its edge meets the edge of the red circle in $C$ and $C^{\prime}$, points on opposite sides of $A B$. Place the next two dises so that in each case $A P$ is a chord of the disc. Suppose that these discs meet the edge of the red circle in $D$ and $D^{\prime}$ respectively, $C$ and $D$ being on the same side of $A B$. Then if we place the two remaining discs so that $C D$ and $C^{\prime} D^{\prime}$ are chords of them, the problem will be solved. The minimum discs are not obtained, as one might at first guess, by the overlapping of five dises placed at the vertices of an inscribed pentagon.

In practice and for simplicity it is desirable to make the tin discs a trifle larger than the theory requires, and to treat $P$ as coincident with $O$.

The mathematical discussion on this problem is too technical and long to insert here. Probably a bare statement of the results such as is given above, is all that most readers will want. Should closer approximations be desired, here they are*. If the radius of the red circle be taken as one foot, the problem is impossible if the radius of each disc is less than 609180 foot, and is soluble if it exceeds 609185 foot. Also $O P=\cdot 028545$ foot, hence $O$ lies very near but not quite on the circumference of the first disc

* See Solutions of Numerical Functional Equations, by E. H. Neville, Proceedings of the London Mathematical Society, 1915, second series, vol. xiv, pp. 308-326. 
which is put down. If three of the dises are placed so that their edges pass through $O$, the radius of each of them must exceed 6099578 foot, a length practically indistinguishable from that of the radius of the minimum disc. If the discs are put with their centres at the vertices of an inscribed pentagon and their edges passing through $O$, the radius of each of them must exceed - 6180340 foot. It follows that unless the discs are cut with extreme accuracy the problem may be solved by making the circumference of each disc pass through $O$; the possibility of using this inaccurate rule is a serious defect in the problem when used as the foundation of a puzzle.

I believe that the discs used in fairs are generally large enough to allow of the employment of the inaccurate rule, though even then it is safer to use the correct method. In an example made for myself I put a minute faint mark near the centre of the red circle but just far enough away to ensure failure for those who make that point lie on the edge of each disc. Notwithstanding their neglect or ignorance of the correct rule showmen seem to find that the game is profitable, and obviously this is an excellent test of its merits from their point of view.

Tesselation with Super-Dominoes. A novel form of tesselation problem was suggested in 1921 by Major MacMahon*. The object is to fill, according to certain rules, a prescribed area with wood or cardboard super-dominoes. The shape of an ordinary domino is a rectangle, the breadth of which is immaterial, with two ends or faces on which numbers or pips can be inscribed, and if we like each number may be taken to indicate a particular colour; by using $n$ numbers we can get $n(n+1) / 2$ different linear dominoes. If our dominoes are triangular in shape, and from the centres of the triangles we draw lines to the angular points, we get dominoes with three faces on each of which numbers or pips can be inscribed, and as before each number may be taken to indicate a particular colour: by using $n$ colours we can get $n\left(n^{2}+2\right) / 3$ different triangular dominoes. Similarly, by using $n$ colours we can get $n(n+1)\left(n^{2}-n+2\right) / 4$ different square

- P. A. MacMahon, New Mathematical Pastimes, Cambridge, 1921. 
dominoes, $n\left(n^{4}+1\right) / 5$ different pentagonal dominoes, and so on. With each of these sets we can make up puzzles.

A particular case will illustrate the kind of questions treated. Take the case of equilateral triangular dominoes. If four colours are used we get a set of twenty-four different dominoes, and these can be put together (preferably in a shallow box cut to the right size) to make a regular hexagon. Innumerable conditions may be imposed. For example, we may suppose that it is required to fit the dominoes into the box so that the colours of adjoining faces of the dominoes shall be the same, as also those of all the exterior faces. Here is the solution of this problem. A consideration of what conditions may be imposed

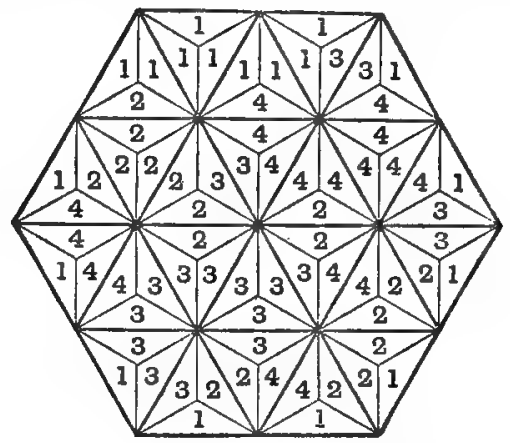

naturally arises, and leads to various other problems. If our dominoes are right-angled triangles we get a hexagon arrangement of a different shape. Further we can play with sets selected from a particular full set and arranged in other geometrical figures. Similar problems arise from the use of square dominoes, hexagonal dominoes, etc.

Geometrical Dissections. Problems requiring the division by straight lines of a given plane rectilinear figure into pieces which can be put together in some other assigned form are well known. A class of Geometrical Recreations is concerned with such constructions.

Pythagorean Extension. A familiar instance is found in many text-books in a dissection proof of the Pythagorean property of 
a right-angled triangle. In fact, however, if we are given any two squares we can always by three cuts divide them into five pieces which can be put together to make a square. There are various solutions. Here are two which answer the purpose*.

The first of these is as follows: Place the larger and smaller squares $A G$ and $C E$ side by side as in figure $i$ below, and take $A B$ equal to $C D$. Then a cut $B H$ and a cut $B E$ (i.e. a cut $B J$ on the larger square and another cut $J E$ on the smaller square) will divide the squares into five pieces which can be put together to make one square of which $B H$ and $B E$ are sides.

A more symmetrical, though less simple, five-part solution (made up of the smaller square together with a four-part division

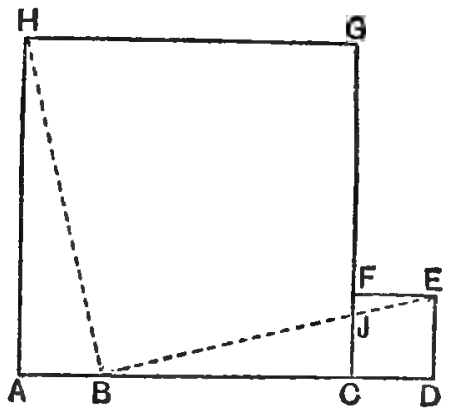

Figure i.

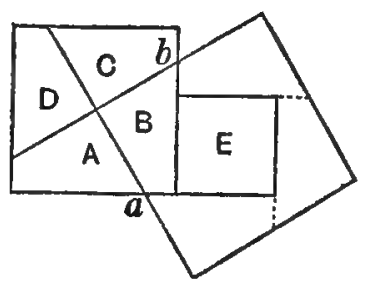

Figure ii.

of the larger square) can be effected as follows. Place the larger square (denoted by the letters $A, B, C, D$ ) next the smaller square (denoted by $E$ ), as in figure ii, with their bases in the same line. Bisect this line in one point $a$ and on their common side take another point $b$ whose distance above their bases is half the sum of their sides. Through these points draw lines perpendicular to each other, crossing in the centre of the larger square, and terminating in its sides. This divides the larger square into four equal parts $A, B, C, D$. Produce the two lines drawn through $a$ and $b$ for half their length beyond the common base and side of the squares, and through their extremities draw two other lines perpendicular to them. These four lines will form another

* H. Perigal, Messenger of Mathematics, 1873, vol. ii, N.S. pp. 103-106; H. E. Dudeney, Amusements, London, 1917, p. 32.

B. $R$. 
square inclosing the small square within four segments equal and similar to the four quarters of the larger of the given squares, and consequently equal in area to the two together.

Montucla's Dissection. Demonstrations of a few similar propositions had long been current, but towards the close of the eighteenth century attention was recalled to this kind of solution by Monucla who proposed and solved the problem of dividing a rectangle so that the parts could be put together in the form of a square; he also solved the converse problem. Later, other solutions of this problem were given by $\mathrm{P}$. Busschop and de Coatpont who made respectively eight-part and seven-part dissections. The former also had constructions for making a square from a five-part division of a regular hexagon, and from a seven-part division of a regular pentagon*.

Polygonal Dissections. The more general problem of the dissection of a given polygon of any number of sides, and the rearrangement of the parts in the form of another polygon of equal area, had been raised by Bolyai, and a method of solution indicated by Gerwien. The question continued to attract occasional attention. In particular a solution for the case of a polygon and a triangle was given by Euzet in 1854, and the wider problem of two polygons was discussed by E. Guitel in 1895, and by $\mathrm{E}$. Holst in 1896 , the results 'being obtained by triangular reductions $\uparrow$.

Minimum Dissections. The above mentioned writers aimed only at finding a solution, and did not in general trouble themselves with considering the smallest number of pieces required. In 1905, the special cases of a four-part dissection of a pair of triangles, a four-part dissection of a triangle and parallelogram, and dissections of a pair of parallelograms were given by $\mathbf{H}$. M.

* G. G. F. Montucla, Ozanan's Récréations Mathematiques, 1803, English edition, vol. i, pp. 292-298, 1840 edition, pp. 127-129; Paul Busschop, Nouvelle Correspondance Mathématique, Brussels, 1875, vol. ii, p. 83; de Coatpont, Ibid. 1876, vol. iii, p. 116.

† P. Gerwien, Crelle's Journal, 1833, p. 228; M. Euzet, Nonvelles Annales de Mathématiques, 1854, vol. siii, pp. 114-115; E. Guitel, Association Française pour l'avancement des sciences, 1895, pp. 264-267; E. Holst, L'Intermédiaire des Mathématiciens, 1896, rol. iii, pp. 91-92. 
Taylor*, and he recognised the desirability of finding the smallest necessary number of cuts.

Henceforth the explicit determination of the number of pieces in which a figure has to be cut to give a solution was regarded as an essential feature of the problem, and it is accepted that that solution is to be preferred in which the number of pieces is smallest. I have no doubt that Taylor's solutions do in fact give the minimum number of pieces required in those cases.

Puzzle Dissections. Of late years H. E. Dudeney has propounded various ingenious puzzles of this kind, in all cases the number of parts being specified t. His reputation has served to attract attention to this class of problems. As illustrating his results and as geometrical recreations of this type, I pick out his problems, $(a)$ to divide, by two straight lines, a Greek cross, that is, one made up of five equal squares, into four pieces all of the same shape and size which can be put together to make a Greek cross; (b) to divide an isosceles right-angled triangle into four pieces which can be put together to make a Greek cross: (c) to divide a regular pentagon into six pieces which can be put together to make a square; (d) to divide an equilateral triangle into four pieces which can be put together to make a square. The reader interested in the subject will like to compare Dudeney's solution of the last question with that given by Taylor already mentioned, and that by Macaulay referred to below.

Dudeney has also dealt with a few problems (to me, personally, less interesting) in which more than two figures are concerned, such as to divide a square into four pieces that will make two equal Greek crosses; and to divide a Greek cross into five pieces which will make two equal Greek crosses.

Macaulay's Four-part Dissections. Recently the theory of four-part dissections of pairs of rectilinear figures of equal area has been discussed by W. H. Macaulaył. He has treated four-

* Messenger of Mathematics, vol. xxxv, pp. 81-101.

+ For instance, see his Amusements in Mathematics, London, 1917, p. 27 et seq.

$\doteqdot$ Mathematical Gazette, 1914, vol. vii, p. 381; vol. viii, 1915, pp. 72, 109; Messenger of Mathematics, vol. xlviii, 1919, p. 159; vol. xlix, 1919, p. 111. 
part dissections of pairs of triangles, of a triangle and a parallelogram, of pairs of quadrilaterals, of pairs of pentagons each with two sides equal and parallel, and of pairs of certain related hexagons. His results are projective and all of them are deducible from his hexagon dissections. This is an interesting generalisation.

The solutions of these problems are lengthy, and almost necessarily involve several diagrams. I content myself with the above notes and references.

The Window Reader. Some years ago a set of eight numbered and perforated cards was brought out which enabled an operator to state a number chosen by a spectator. Of the eight cards each of the first seven was pierced with windowlike openings, each of the last seven contained some of the numbers less than 100 headed by the word $Y e s$, each of the last three had also certain digits on its back, and the first of the cards was headed with the word Top. Each card if turned upside down bore on what was then its top the word $N o$.

The cards were employed to determine any number less than 100 chosen by some one, say, $A$. They were used by $B$ thus. $B$ first laid on the table the card numbered 1 with the side marked Top uppermost. $B$ then took the second card, and asked $A$ if the chosen number was on it; if $A$ said yes, $B$ placed the card 2 on the top of the card 1 with the $Y e s$ uppermost; and if $A$ said no, $B$ turned the card round and placed it with the No uppermost. $B$ then asked if the chosen number was on the third card, and placed it on the top of 2 with the appropriate end uppermost; and so on with the rest of the cards 4 to 8 . Finally on turning the whole pile over, the chosen number was seen through the windows.

The puzzle must have been widely circulated. It was sold in Italy and in London, and friends tell me that they also saw it in Germany. The method used is fairly obvious, and I will leave to any reader, sufficiently interested, the task of constructing cards suitable for the purpose.

Evidently, howeyer, any number not exceeding 128 can be determined by only seven cards, each bearing 64 selected 
numbers. For the first card serves to divide the numbers into two sets of 64 numbers, numbers on the second card can be chosen so as to divide each of these into two sets of 32 cards, the third divides each of these into two sets of 16 cards, and so on. The numbers must be written on the cards and the windows cut so that after arranging the cards in their proper order, and turning the pack over, the chosen number appears on the back of the seventh card as seen through the windows cut in the first six cards. To arrange the numbers in this way presents no difficulty, but the geometrical problem of cutting the windows is less easy. I give one solution.

If we work with seven cards one way of preparing them is as follows. We write under the word Yes on the first card, the 64 numbers 1 to 32,65 to 96 ; on the second card, the numbers 1 to 16,33 to 48,65 to 80,97 to 112 ; on the third card, the numbers in four arithmetical progressions starting from $1,2,5,6$, each of 16 terms, with 8 as difference; on the fourth card, the numbers in eight arithmetical progressions starting from 1, 2, $3,4,5,6,7,8$, each of 8 terms, with 16 as difference; on the fifth card, the odd numbers from 1 to 127; on the sixth card, the numbers in four arithmetical progressions starting from $1,2,3,4$, each of 16 terms, with 8 as difference; and on the seventh card the consecutive numbers from 1 to 64 , on this card however the No must be written on the left hand edge of the card and not on its lowest edge. On the back of the last card we must now write the numbers from 1 to 128 in their natural order, 1 to 32 occupying the first quarter, 33 to 64 the fourth quarter, 65 to 96 the second quarter rotated through a right angle, and 97 to 128 the third quarter also rotated through a right angle. The spacing of the figures requires care, but is not difficult if the principle of construction is grasped, and squared paper used.

The windows should be cut as follows. We will suppose that we use squared paper dividing each card into four equal quadrants with margins. In the first card, we form the window by cutting out the whole of the second quadrant. In the second card, we get two windows by cutting out the top half of the second 
quadrant and the top half of the third quadrant. In the third card, we get two windows by cutting out the right half of the second quadrant and the right half of the third quadrant. In the fourth card, we divide the second and third quadrants into four equal horizontal strips and from each of these quadrants cut out the first and third strips. In the fifth card, we divide the second and third quadrants into four equal vertical strips, and from each of these quadrants cut out the second and fourth of these strips. In the sixth card we divide the second and third quadrants into eight horizontal strips and from each of these quadrants cut out the first, third, fifth, and seventh strips.

It will be noticed that no windows are cut in the first or fourth quarter of any card; hence they are free for insertion of the 64 numbers written on the face of each card. The construction here given is due to my friend R. A. L. Cole.

Possibly the puzzle is better presented by omitting all numbers exceeding 100, for the introduction of 128 at once suggests the method of construction. With that restriction I think the use of only seven cards is better and more elegant than the form in which I have seen it on sale.

Compass Problems. It is well known that Euclid in his Elements confined his constructions to those which could be made with ungraduated rulers and compasses. The use of a ruler is however unnecessary in many cases, and Mascheroni* established a number of propositions by constructions made with compasses alone. He presented his propositions as a connected series, and of course the logical sequence is very different from that with which we are fumiliar. It is remarkable how much he effected without being able to join points by straight lines or make use of properties of parallels.

As an instance which will illustrate the subject, I select the problem to find a point midway between two given points $A$ and $B$. Of this fundamental proposition Mascheroni gave five solutions (prop. 66). Here are two of them: they rest on the assump-

* His work was first published at Bergamo, 1782. French translations were issued at Paris in 1798 and 1828, and from these my knowledge of the book is derived. 
tion that we can draw a semicircle whose centre and one extremity are given, a result he had previously established. In each case the demonstration is straightforward. Other solutions of this proposition have been given, some of which evade the use of a semicircle.

One of his constructions is as follows. With $B$ as centre, and a radius $B A$, describe a semicircle of which $A$ and $C$ are the extremities. With centres $A$ and $C$, and radii $A B$ and $C A$, describe circles which cut in $P$ and $Q$. With centres $P$ and $Q$, and a radius equal to $A B$, describe circles. These will cut in a point midway between $A$ and $B$.

Here is another of his solutions, which for some purposes he preferred. With $B$ as centre, and radius $B A$, describe a semicircle of which $A$ and $C$ are the extremities. With $A$ and $C$ as centres, and a radius equal to $A B$, describe circles which cut the semicircle in $H$ and $K$ respectively. With $A$ and $C$ as centres, and a radius equal to $A C$, describe circles which cut the last mentioned circles (above $A C$ ) in $Q$ and $P$ respectively. With centres $P$ and $C$, and radii $P A$ and $P Q$, describe circles. These will cut in a point midway between $A$ and $B$.

Numerous geometrical recreations of this kind can be made by any one, for all that is necessary is to select at random one of Mascheroni's propositions, and see how it can be established by using only circles. As instances, I select the construction on a given line of a triangle similar to a given triangle (prop. 125); and the construction of a regular pentagon of given dimensions (prop. 137). Whatever be the solution obtained, it is always interesting to turn to Mascheroni's book, and see how the question is tackled there. 


\section{CHAPTER XIII.}

\section{CALCUlating PRODigies.}

At rare intervals there have appeared lads who possess extraordinary powers of mental calculation*. In a few seconds they gave the answers to questions connected with the multiplication of numbers and the extraction of roots of numbers, which an expert mathematician could obtain only in a longer time and with the aid of pen and paper. Nor were their powers always limited to such simple problems. More difficult questions, dealing for instance with factors, compound interest, annuities, the civil and ecclesiastical calendars, and the solution of equations, were solved by some of them with facility as soon as the meaning of what was wanted had been grasped. In most cases these lads were illiterate, and usually their rules of working were of their own invention.

The performances were so remarkable that some observers held that these prodigies possessed powers differing in kind from those of their contemporaries. For such a view there is no foundation. Any lad with an excellent memory and a natural turn for arithmetic can, if he continuously gives his undivided attention to the consideration of numbers, and indulges in constant practice, attain great proficiency in mental arithmetic, and of course the performances of those that are specially gifted are exceptionally astonishing.

* Most of the facts about calculating prodigies have been collected by E. W. Scripture, American Journal of Psychology, 1891, vol. Iv, pp. 1-59; by F. D. Mitchell, Ibid., 1907, vol. xvirI, pp. 61-143; and G. W. Müller, Zur Analyse der Gedächtnistätigkeit und des Vorstellungsverlaufes, Leipzig, 1911. I have used these papers freely, and in some cases where authorities are quoted of which I have no first-hand information have relied exclusively on them. These articles should be consulted for bibliographical notes on the numerous original authorities. 
In this chapter I propose to describe briefly the doings of the more famous calculating prodigies. It will be seen that their performances were of much the same general character, though carried to different extents, hence in the later cases it will be enough to indicate briefly peculiarities of the particular calculators.

I confine myself to self-taught calculators, and thus exclude the consideration of a few public performers who by practice, arithmetical devices, and the tricks of the showman have simulated like powers. I also concern myself only with those who showed the power in youth. As far as I know the only self-taught mathematician of advanced years whom $I$ thus exclude is John Wallis, 1616-1703, the Savilian Professor at Oxford, who in middle-life developed, for his own amusement, his powers in mental arithmetic. As an illustration of his achievements, I note that on 22 December 1669 he, when in bed, occupied himself in finding (mentally) the integral part of the square root of $3 \times 10^{40}$; and several hours afterwards wrote down the result from memory. This fact having attracted notice, two months later he was challenged to extract the square root of a number of fifty-three digits; this he performed mentally, and a month later he dictated the answer which he had not meantime committed to writing. Such efforts of calculation and memory are typical of calculating prodigies.

One of the earliest of these prodigies, of whom we have records, was Jedediah Buxton, who was born in or about 1707 at Elmton, Derbyshire. Although a son of the village schoolmaster, his education was neglected, and he never learnt to write or cipher. With the exception of his power of dealing with large numbers, his mental faculties were of a low order: he had no ambition, and remained throughout his life a farmlabourer, nor did his exceptional skill with figures bring him any material advantage other than that of occasionally receiving small sums of money from those who induced him to exhibit his peculiar gift. He does not seem to have given public exhibitions. He died in 1772 .

$\mathrm{He}$ had no recollection as to when or how he was first 
attracted by mental calculations, and of his performances in early life we have no reliable details. Mere numbers however seem always to have had a strange fascination for him. If the size of an object was stated, he began at once to compute how many inches or hair-breadths it contained; if a period of time was mentioned, he calculated the number of minutes in it; if he heard a sermon, he thought only of the number of words or syllables in it. No doubt his powers in these matters increased by incessant practice, but his ideas were childish, and do not seem to have gone beyond pride in being able to state accurately the results of such calculations. He was slow witted, and took far longer to answer arithmetical questions than most of these prodigies. The only practical accomplishment to which his powers led him was the ability to estimate by inspection the acreage of a field of irregular shape.

His fame gradually spread through Derbyshire, Among many questions put to him by local visitors were the following, which fairly indicate his powers when a young man:How many acres are there in a rectangular field 351 yards long and 261 wide; answered in 11 minutes. How many cubic yards of earth must be removed in order to make a pond 426 feet long, 263 feet wide, and $2 \frac{1}{2}$ feet deep; answered in 15 minutes. If sound travels 114,2 feet in one second, how long will it take to travel 5 miles; answered in 15 minutes. Such questions involve no difficulties of principle.

Here are a few of the harder problems solved by Buxton when his powers were fully developed. He calculated to what sum a farthing would amount if doubled 140 times: the answer is a number of pounds sterling which requires thirty-nine digits to represent it with $2 s$. $8 d$. over. He was then asked to multiply this number of thirty-nine digits by itself : to this he gave the answer two and a half months later, and he said he had carried on the calculation at intervals during that period. In 1751 he calculated how many cubic inches there are in a right-angled block of stone 23,145,789 yards long, 5,642,732 yards wide, and 54,965 yards thick; how many grains of corn would be required to fill a cube whose volume is $202,680,000,360$ cubic miles; and 
how many hairs one inch long would be required to fill the same space-the dimensions of a grain and a hair being given. These problems involve high numbers, but are not intrinsically difficult, though they could not be solved mentally unless the calculator had a phenomenally good memory. In each case he gave the correct answer, though only after considerable effort. In 1753 he was asked to give the dimensions of a cubical cornbin, which holds exactly one quarter of malt. He recognized that to answer this required a process equivalent to the extraction of a cube root, which was a novel idea to him, but in an hour he said that the edge of the cube would be between $25 \frac{1}{2}$ and 26 inches, which is correct: it has been suggested that he got this answer by trying various numbers.

Accounts of his performances were published, and his reputation reached London, which he visited in 1754. During his stay there he was examined by various members of the Royal Society, who were satisfied as to the genuineness of his performances. Some of his acquaintances took him to Drury Lane Theatre to see Garrick, being curious to see how a play would impress his imagination. He was entirely unaffected by the scene, but on coming out informed his hosts of the exact number of words uttered by the various actors, and of the number of steps taken by others in their dances.

It was only in rare cases that he was able to explain his methods of work, but enough is known of them to enable us to say that they were clumsy. He described the process by which he arrived at the product of 456 and 378 : shortly it was as follows:-If we denote the former of these numbers by $a$, he proceeded first to find $5 a=$ (say) $b$; then to find $20 b=$ (say) $c$; and then to find $3 c=$ (say) $d$. He next formed $15 b=$ (say) $e$, which he added to $d$. Lastly he formed $3 a$ which, added to the sum last obtained, gave the result. This is equivalent to saying that he used the multiplier 378 in the form $(5 \times 20 \times 3)+(5 \times 15)+3$. Mitchell suggests that this may mean that Buxton counted by multiples of 60 and of 15 , and thus reduced the multiplication to addition. It may be so, for it is difficult to suppose that he did not realize that successive 
multiplications by 5 and 20 are equivalent to a multiplication by 100 , of which the result can be at once obtained. Of billions, trillions, \&c., he had never heard, and in order to represent the high numbers required in some of the questions proposed to him he invented a notation of his own, calling $10^{18}$ a tribe and $10^{36} \mathrm{a}$ cramp.

As in the case of all these calculators, his memory was exceptionally good, and in time he got to know a large number of facts (such as the products of certain constantly recurring numbers, the number of minutes in a year, and the number of hair-breadths in a mile) which greatly facilitated his calculations. A curious and perhaps unique feature in his case was that he could stop in the middle of a piece of mental calculation, take up other subjects, and after an interval, sometimes of weeks, could resume the consideration of the problem. He could answer simple questions when two or more were proposed simultaneously.

Another eighteenth-century prodigy was Thomas Fuller, a negro, born in 1710 in Africa. He was captured there in 1724, and exported as a slave to Virginia, U.S.A., where he lived till his death in 1790. Like Buxton, Fuller never learnt to read or write, and his abilities were confined to mental arithmetic. He could multiply together two numbers, if each contained not more than nine digits, could state the number of seconds in a given period of time, the number of grains of corn in a given mass, and so on-in short, answer the stock problems commonly proposed to these prodigies, as long as they involved only multiplications and the solutions of problems by rule of three. Although more rapid than Buxton, he was a slow worker as compared with some of those whose doings are described below.

I mention next the case of two mathematicians of note who showed similar aptitude in early years. The first of these was André Marie Ampère, 1775-1836, who, when a child some four years old, was accustomed to perform long mental calculations, which he effected by means of rules learnt from playing with arrangements of pebbles. But though always expert at mental arithmetic, and endowed with a phenomenal memory for figures, 
he did not specially cultivate this arithmetical power. It is more difficult to say whether Carl Friedrich Gauss, 1777-1855, should be reckoned among these calculating prodigies. He had, when three years old, taught himself some arithmetical processes, and astonished his father by correcting him in his calculations of certain payments for overtime; perhaps, however, this is only evidence of the early age at which his consummate abilities began to develop. Another remarkable case is that of Richard Whately, 1787-1863, afterwards Archbishop of Dublin. When he was between five or six years old he showed considerable skill in mental arithmetic: it disappeared in about three years. I soon, said he, "got to do the most difficult sums, always in my head, for I knew nothing of figures beyond numeration, nor had I any names for the different processes I employed. But I believe my sums were chiefly in multiplication, division, and the rule of three...I did these sums much quicker than any one could upon paper, and I never remember committing the smallest error. I was engaged either in calculating or in castle-building...morning, noon, and night... When I went to school, at which time the passion was worn off, I was a perfect dunce at ciphering, and so have continued ever since." The archbishop's arithmetical powers were, however, greater in after-life than he here allows.

The performances of Zerah Colburn in London, in 1812, were more remarkable. Colburn*, born in 1804, at Cabut, Vermont, U.S.A., was the son of a small farmer. While still less than six years old he showed extraordinary powers of mental calculation, which were displayed in a tour in America. Two years later he was brought to England, where he was repeatedly examined by competent observers. He could instantly give the product of two numbers each of four digits, but hesitated if both numbers exceeded 10,000. Among questions asked him at this time were to raise 8 to the 16 th power; in a few seconds he gave the answer $281,474,976,710,656$, which is correct. He was next asked to raise the numbers $2,3, \ldots 9$ to

* To the authorities mentioned by E. W. Scripture and F. D. Mitchell should be added The Annual Register, London, 1812, p. 507 et seq. 
the 10th power: and he gave the answers so rapidly that the gentleman who was taking them down was obliged to ask him to repeat them more slowly; but he worked less quickly when asked to raise numbers of two digits like 37 or 59 to high powers. He gave instantaneously the square roots and cube roots (when they were integers) of high numbers, e.g., the square root of 106,929 and the cube root of $268,336,125$, such integral roots can, however, be obtained easily by various methods. More remarkable are his answers to questions on the factors of numbers. Asked for the factors of 247,483 he replied 941 and 263 ; asked for the factors of 171,395 he gave $5,7,59$, and 83 ; asked for the factors of 36,083 he said there were none. He, however, found it difficult to answer questions about the factors of numbers higher than $1,000,000$. His power of factorizing high numbers was exceptional and depended largely on the method of two-digit terminals described below. Like all these public performers he had to face buffoons who tried to make fun of him, but he was generally equal to them. Asked on one such occasion how many black beans were required to make three white ones, he is said to have at once replied "three, if you skin them"this, however, has much the appearance of a pre-arranged show.

It was clear to observers that the child operated by certain rules, and during his calculations his lips moved as if he was expressing the process in words. Of his honesty there seems to have been no doubt. In a few cases he was able to explain the method of operation. Asked for the square of 4,395 he hesitated, but on the question being repeated he gave the correct answer, namely 19,395,025. Questioned as to the cause of his hesitation, he said he did not like to multiply four figures by four figures, but said be, "I found out another way; I multiplied 293 by 293 and then multiplied this product twice by the number 15." On another occasion when asked for the product of 21,734 by 543 he immediately replied 11,801,562; and on being questioned explained that he had arrived at this by multiplying 65,202 by 181 . These remarks suggest that whenever convenient he factorized the numbers with which he was dealing. 
In 1814 he was taken to Paris, but amid the political turmoil of the time his exhibitions fell flat. His English and American friends however raised money for his education, and he was sent in succession to the Lycée Napoleon in Paris and Westminster School in London. With education his calculating powers fell off, and he lost the frankness which when a boy had charmed observers. His subsequent career was diversified and not altogether successful. He commenced with the stage, then tried schoolmastering, then became an itinerant preacher in America, and finally a "professor" of languages. He wrote his own biography which contains an account of the methods he used. He died in 1840 .

Contemporary with Colburn we find another instance of a self-taught boy, George Parker Bidder, who possessed quite exceptional powers of this kind. $H e$ is perhaps the most interesting of these prodigies because he subsequently received a liberal education, retained his calculating powers, and in later life analyzed and explained the methods he had invented and used.

Bidder was born in 1806 at Moreton Hampstead, Devonshire, where his father was a stone-mason. At the age of six he was taught to count up to 100 , but though sent to the village school learnt little there, and at the beginning of his career was ignorant of the meaning of arithmetical terms and of numerical symbols. Equipped solely with this knowledge of counting he taught himself the results of addition, subtraction, and multiplication of numbers (less than 100) by arranging and rearranging marbles, buttons, and shot in patterns. In after-life he attached great importance to such concrete representations, and believed that his arithmetical powers were strengthened by the fact that at that time he knew nothing about the symbols for numbers. When seven years old he heard a dispute between two of his neighbours about the price of something which was being sold by the pound, and to their astonishment remarked that they were both wrong, mentioning the correct price. After this exhibition the villagers delighted in trying to pose him with arithmetical problems. 
His reputation increased and, before he was nine years old, his father found it profitable to take him about the country to exhibit his powers. A couple of distinguished Cambridge graduates (Thomas Jephson, then tutor of St John's, and John Herschel) saw him in 1817, and were so impressed by his general intelligence that they raised a fund for his education, and induced his father to give up the rôle of showman; but after a few months Bidder senior repented of his abandonment of money so easily earned, insisted on his son's return, and began again to make an exhibition of the boy's powers. In 1818, in the course of a tour young Bidder was pitted against Colburn and on the whole proved the abler calculator. Finally the father and son came to Edinburgh, where some members of that University intervened and persuaded his father to leave the lad in their care to be educated. Bidder remained with them, and in due course graduated at Edinburgh, shortly afterwards entering the profession of civil engineering in which he rose to high distinction. He died in 1878.

With practice Bidder's powers steadily developed. His earlier performances seem to have been of the same type as those of Buxton and Colburn which have been already described. In addition to answering questions on products of numbers and the number of specified units in given quantities, he was, after 1819, ready in finding square roots, cube roots, \&c. of high numbers, it being assumed that the root is an integer, and later explained his method which is easy of application: this method is the same as that used by Colburn. By this time he was able also to give immediate solutions of easy problems on compound interest and annuities which seemed to his contemporaries the most astonishing of all his feats. In factorizing numbers he was less successful than Colburn and was generally unable to deal at sight with numbers higher than 10,000. As in the case of Colburn, attempts to be witty at his expense were often made, but he could hold his own. Asked at one of his performances in London in 1818, how many bulls' tails were wanted to reach to the moon, he immediately answered one, if it is long enough. 
Here are some typical questions put to and answered by him in his exhibitions during the years 1815 to 1819 - they are taken from authenticated lists which comprise some hundreds of such problems: few, if any, are inherently difficult. His rapidity of work was remarkable, but the time limits given were taken by unskilled observers and can be regarded as only approximately correct. Of course all the calculations were mental without the aid of books, pencil, or paper. In 1815, being then nine years old, he was asked:-If the moon be distant from the earth 123,256 miles, and sound travels at the rate of 4 miles a minute, how long would it be before the inhabitants of the moon could hear of the battle of Waterloo: answer, 21 days, 9 hours, 34 minutes, given in less than one minute. In 1816, being then ten years old, just learning to write, but unable to form figures, he answered questions such as the following:-What is the interest on $£ 11,111$ for 11,111 days at

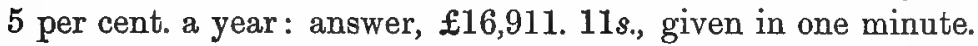
How many hogsheads of cider can be made from a million of apples, if 30 apples make one quart: answer, 132 hogsheads, 17 gallons, 1 quart, and 10 apples over, given in 35 seconds. If a coach-wheel is 5 feet 10 inches in circumference, how many times will it revolve in running 800,000,000 miles: answer, $724,114,285,704$ times and 20 inches remaining, given in 50 seconds. What is the square root of $119,550,669,121$ : answer 345,761, given in 30 seconds. In 1817, being then eleven years old, he was asked:- - How long would it take to fill a reservoir whose volume is one cubic mile if there flowed into it from a river 120 gallons of water a minute: answered in 2 minutes. Assuming that light travels from the sun to the earth in 8 minutes, and that the sun is $98,000,000$ miles off, if light takes 6 years 4 months travelling from the nearest fixed star to the earth, what is the distance of that star, reckoning 365 days 6 hours to each year and 28 days to each monthasked by Sir William Herschel: answer, 40,633,740,000,000 miles. In 1818, at one of his performances, he was asked:-If the pendulum of a clock vibrates the distance of $9 \frac{3}{4}$ inches in a second of time, how many inches will it vibrate in 7 years 14 days

B. $\mathrm{R}$. 
2 hours 1 minute 56 seconds, each vear containing 365 days 5 hours 48 minutes 55 seconds : answer, 2,165,625,744, $\frac{8}{4}$ inches, given in less than a minute. If I have 42 watches for sale and I sell the first for a farthing, and double the price for every succeeding watch I sell, what will be the price of the last watch:

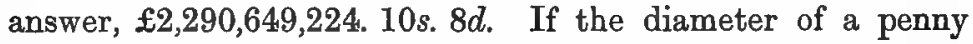
piece is $1 \frac{3}{8}$ inches, and if the world is girdled with a ring of pence put side by side, what is their value sterling, supposing the distance to be 360 degrees, and a degree to contain 69.5 miles: answer, $\mathfrak{1 4}, 803,340$, given in one minute. Find two numbers, whose difference is 12 , and whose product, multiplied by their sum, is equal to 14,560 : answer, 14 and 26. In 1819, when fourteen years old, he was asked:-Find a number whose cube less 19 multiplied by its cube shall be equal to the cube of 6 : answer, 3 , given instantly. What will it cost to make a road for 21 miles 5 furlongs 37 poles 4 yards, at the rate of $£ 123.14 s .6 d$. a mile: answer, $£ 2688$. 13s. $9 \frac{3}{4} d$., given in 2 minutes. If you are now 14 years old and you live 50 years longer and spend half-a-crown a day, how many farthings will you spend in your life: answer, 2,805,120, given in 15 seconds. Mr Moor contracted to illuminate the city of London with 22,965,321 lamps, the expense of trimming and lighting was 7 farthings a lamp, the oil consumed was $\frac{z}{9}$ ths of a pint for every three lamps, and the oil cost $3 s$. $7 \frac{1}{2} d$. a gallon; he gained

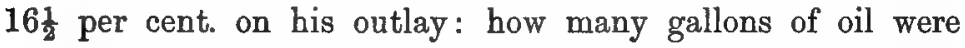
consumed, what was the cost to him, and what was the amount of the contract : answer, he used 212,641 gallons of oil, the cost was $£ 205,996.16 s .1 \frac{s}{4} d$, and the amount of the contract was £239,986. 13s. $2 d$. If the distance of the earth from the moon be $29,531,531 \frac{1}{4}$ yards, what is the weight of a thread which will extend that distance, supposing $7 \frac{11}{16}$ yards of it weigh $\frac{1}{16}$ th part of a drachm: answer, $8 \mathrm{cwt} .1 \mathrm{qr} .13 \mathrm{lbs} .9 \mathrm{oz} .1 \mathrm{dr}$. and $\frac{13}{16}$ ths of a drachm.

It should be noted that Bidder did not visualize a number like 984 in symbols, but thought of it in a concrete way as so many units which could be arranged in 24 groups of 41 each. It should also be observed that he, like Inaudi whom I mention 
later, relied largely on the auditory sense to enable him to recollect numbers. "For my own part," he wrote, in later life, "though much accustomed to see sums and quantities expressed by the usual symbols, yet if $I$ endeavour to get any number of figures that are represented on paper fixed in my memory, it takes me a much longer time and a very great deal more exertion than when they are expressed or enumerated verbally." For instance suppose a question put to find the product of two numbers each of nine digits, if they were "read to me, I should not require this to be done more than once; but if they were represented in the usual way, and put into my hands, it would probably take me four times to peruse them before it would be in my power to repeat them, and after all they would not be impressed so vividly on my imagination."

Bidder retained his power of rapid mental calculation to the end of his life, and as a constant parliamentary witness in matters connected with engineering it proved a valuable accomplishment. Just before his death an illustration of his powers was given to a friend who talking of then recent discoveries remarked that if 36,918 waves of red light which only occupy one inch are required to give the impression of red, and if light travels at 190,000 miles a second, how immense must be the number of waves which must strike the eye in one second to give the impression of red. "You need not work it out," said Bidder, "the number will be $444,433,651,200,000$."

Other members of the Bidder family have also shown exceptional powers of a similar kind as well as extraordinary memories. Of Bidder's elder brothers, one became an actuary, and on his books being burnt in a fire he rewrote them in six months from memory but, it is said, died of consequent brain fever; another was a Plymouth Brother and knew the whole Bible by heart, being sable to give chapter and verse for any text quoted. Bidder's eldest son, a lawyer of eminence, was able to multiply together two numbers each of fifteen digits. Neither in accuracy nor rapidity was he equal to his father, but then he never steadily and continuously devoted himself to developing his abilities in this direction. He remarked 
that in his mental arithmetic he worked with pictures of the figures, and said "If I perform a sum mentally it always proceeds in a visible form in my mind; indeed I can conceive no other way possible of doing mental arithmetic": this it will be noticed is opposed to his father's method. Two of his children, one son and one daughter representing a third generation, have inherited analogous powers.

I mention next the names of Henri Mondeux, and Vito Mangiamele. Both were born in 1826 in humble circumstances, were sheep-herds, and became when children, noticeable for feats in calculation which deservedly procured for them local fame. In 1839 and 1840 respectively they were brought to Paris where their powers were displayed in public, and tested by Arago, Cauchy, and others. Mondeux's performances were the more striking. One question put to him was to solve the equation $x^{3}+84=37 x$ : to this he at once gave the answer 3 and 4 , but did not detect the third root, namely, -7 . Another question asked was to find solutions of the indeterminate equation $x^{2}-y^{2}=133$ : to this he replied immediately 66 and 67 ; asked for a simpler solution he said after an instant 6 and 13. I do not however propose to discuss their feats in detail, for there was at least a suspicion that these lads were not frank, and that those who were exploiting them had taught them rules which enabled them to simulate powers they did not really possess. Finally both returned to farm work, and ceased to interest the scientific world. If Mondeux was self-taught we must credit him with a discovery of some algebraic theorems which would entitle him to rank as a mathematical genius, but in that case it is inconceivable that he never did anything more, and that his powers appeared to be limited to the particular problens solved by him.

Johann Martin Zacharias Dase, whom I next mention, is a far more interesting example of these calculating prodigies. Dase was born in 1824 at Hamburg. He had a fair education, and was afforded every opportunity to develop his powers, but save in matters connected with reckoning and numbers he made little progress and struck all observers as dull. Of 
geometry and any language but German he remained ignorant to the end of his days. He was trustworthy and filled various small official posts in Germany. He gave exhibitions of his calculating powers in Germany, Austria, and England. He died in 1861.

When exhibiting in Vienna in 1840, he made the acquaintance of Strasznicky who urged him to apply his powers to scientific purposes. This Dase gladly agreed to do, and so became acquainted with Gauss, Schumacher, Petersen, and Encke. To his contributions to science I allude later. In mental arithmetic the only problems to which I find allusions are straightforward examples like the following:-Multiply $79,532,853$ by $93,758,479$ : asked by Schumacher, answered in 54 seconds. In answer to a similar request to find the product of two numbers each of twenty digits he took 6 minutes; to find the product of two numbers each of forty digits he took 40 minutes; to find the product of two numbers each of a hundred digits he took 8 hours 45 minutes. Gauss thought that perhaps on paper the last of these problems could be solved in half this time by a skilled computator. Dase once extracted the square root of a number of a hundred digits in 52 minutes. These feats far surpass all other records of the kind, the only calculations comparable to them being Buxton's squaring of a number of thirty-nine digits, and Wallis' extraction of the square root of a number of fifty-three digits. Dase's mental work however was not always accurate, and once (in 1845) he gave incorrect answers to every question put to him, but on that occasion he had a headache, and there is nothing astonishing in his failure.

Like all these calculating prodigies he had a wonderful memory, and an hour or two after a performance could repeat all the numbers mentioned in it. He had also the peculiar gift of being able after a single glance to state the number (up to about 30) of sheep in a flock, of books in a case, and so on; and of visualizing and recollecting a large number of objects. For instance, after a second's look at some dominoes he gave the sum (117) of their points; asked how many letters were in 
a certain line of print chosen at random in a quarto page he instantly gave the correct number (63); shown twelve digits he had in half a second memorized them and their positions so as to be able to name instantly the particular digit occupying any assigned place. It is to be regretted that we do not know more of these performances. Those who are acquainted with the delightful autobiography of Robert-Houdin will recollect how he cultivated a similar power, and how valuable he found it in the exercise of his art.

Dase's calculations, when also allowed the use of paper and pencil, were almost incredibly rapid, and invariably accurate. When he was sixteen years old Straszmicky taught him the use of the familiar formula $\pi / 4=\tan ^{-1}\left(\frac{1}{2}\right)+\tan ^{-1}\left(\frac{1}{5}\right)+\tan ^{-1}\left(\frac{1}{8}\right)$, and asked him thence to calculate $\pi$. In two months he carried the approximation to 205 places of decimals, of which 200 are correct*. Dase's next achievement was to calculate the natural logarithms of the first $1,005,000$ numbers to 7 places of decimals; he did this in his off-time from 1844 to 1847, when occupied by the Prussian survey. During the next two years he compiled in his spare time a hyperbolic table which was published by the Austrian Government in 1857. Later he offered to make tables of the factors of all numbers from 7,000000 to $10,000,000$ and, on the recommendation of Gauss, the Hamburg Academy of Sciences agreed to assist him so that he might have leisure for the purpose, but he lived only long enough to finish about half the work.

Truman Henry Safford, born in $\mathbf{1 8 3 6}$ at Royalton, Vermont, U.S.A., was another calculating prodigy. He was of a somewhat different type for he received a good education, graduated in due course at Harvard, and ultimately took up astronomy in which subject he held a professional post. I gather that though always a rapid calculator, he gradually lost the exceptional powers shown in his youth. He died in 1901.

Safford never exhibited his calculating powers in public, and I know of them only through the accounts quoted by Scripture

* The result was published in Crelle's Journal, 1844, vol. xxvir, p. 198: on closer approximations and easier formulae, see below chapter xiii. 
and Mitchell, but they seem to have been typical of these calculators. In 1842, he amused and astonished his family by mental calculations. In 1846, when ten years old, he was examined, and here are some of the questions then put to him:-Extract the cube root of a certain number of seven digits; answered instantly. What number is that which being divided by the product of its digits, the quotient is three, and if 18 be added the digits will be inverted: answer 24 , given in about a minute. What is the surface of a regular pyramid whose slant height is 17 feet, and the base a pentagon of which each side is 33.5 feet: answer 3354:5558 square feet, given in two minutes. Asked to square a number of eighteen digits he gave the answer in a minute or less, but the question was made the more easy as the number consisted of the digits 365 repeated six times. Iike Colburn he factorized high numbers with ease. In such examples his processes were empirical, be selected (he could not tell how) likely factors and tested the matter in a few seconds by actual division.

There are to-day four calculators of some note. These are Ugo Zamebone, an Italian, born in 1867 ; Pericles Diamandi, a Greek, born in 1868; Carl Rückle, a German; and Jacques Inaudi, born in 1867. The three first mentioned are of the normal type and I do not propose to describe their performances, but Inaudi's performances merit a fuller treatment.

Jacques Inaudi* was born in 1867 at Onorato in Italy. He was employed in early years as a sheep-herd, and spent the long idle hours in which he had no active duties in pondering on numbers, but used for them no concrete representations such as pebbles. His calculating powers first attracted notice about 1873. Shortly afterwards his elder brother sought his fortune as an organ grinder in Provence, and young Inaudi, accompanying him, came into a wider world, and earned a few coppers for himself by street exhibitions of his powers. His ability was exploited by showmen, and thus in 1880 he visited Paris

* See Charcot and Darboux, Mémoires de l'Institut, Comptes Rendus, 1892, vol. cxrv, pp. 275, 528, 578; and Binet, Rêvue des deux Mondes, 1892, vol. oxr, pp. 905-924. 
where he gave exhibitions: in these he impressed all observers as being modest, frank, and straightforward. He was then ignorant of reading and writing: these arts he subsequently acquired.

His earlier performances were not specially remarkable as compared with those of similar calculating prodigies, but with continual practice he improved. Thus at Lyons in 1873 he could multiply together almost instantaneously two numbers of three digits. In 1874 he was able to multiply a number of six digits by another number of six digits. Nine years later he could work rapidly with numbers of nine or ten digits. Still later, in Paris, asked by Darboux to cube 27, he gave the answer in 10 seconds. In 13 seconds he calculated how many seconds are contained in 18 years 7 months 21 days 3 hours: and he gave immediately the square root of one-sixth of the difference between the square of 4801 and unity. He also calculated with ease the amount of wheat due according to the traditional story to Sessa who, for inventing chess, was to receive 1 grain on the first cell of a chess-board, 2 on the second, 4 on the third, and so on in geometrical progression.

$\mathrm{He}$ can find the integral roots of equations and integral solutions of problems, but proceeds only by trial and error. His most remarkable feat is the expression of numbers less than $10^{5}$ in the form of a sum of four squares, which he can usually do in a minute or two; this power is peculiar to him. Such problems have been repeatedly solved at private performances, but the mental strain caused by them is considerable.

A performance before the general public rarely lasts more than 12 minutes, and is a much simpler affair. A normal programme includes the subtraction of one number of twentyone digits from another number of twenty-one digits: the addition of five numbers each of six digits: the multiplying of a number of four digits by another number of four digits: the extraction of the cube root of a number of nine digits, and of the fifth root of a number of twelve digits: the determination of the number of seconds in a period of time, and the day of the week on which a given date falls. Of course the questions are put by members of the audience. To a pro. 
fessional calculator these problems are not particularly difficult. As each number is announced, Inaudi repeats it slowly to his assistant, who writes it on a blackboard, and then slowly reads it aloud to make sure that it is right. Inaudi then repeats the number once more. By this time he has generally solved the problem, but if he wants longer time he makes a few remarks of a general character, which he is able to do without interfering with his mental calculations. Throughout the exhibition he faces the audience: the fact that he never even glances at the blackboard adds to the effect.

It is probable that the majority of calculating prodigies rely on the speech muscles as well as on the eye and the ear to help them to recollect the figures with which they are dealing. It was formerly believed that they all visualized the numbers proposed to them, and certainly some have done so. Inaudi however trusts mainly to the ear and to articulation. Bidder also relied partly on the ear, and when he visualized a number it was not as a collection of digits but as a concrete collection of units divisible, if the number was composite, into definite groups. Rückle relies mainly on visualizing the numbers. So it would seem that there are different types of the memories of calculators. Inaudi can reproduce mentally the sound of the repetition of the digits of the number in his own voice, and is confused, rather than helped, if the numbers are shown to him in writing. The articulation of the digits of the number also seems necessary to enable him fully to exhibit his powers, and he is accustomed to repeat the numbers aloud before beginning to work on them-the sequence of sounds being important. A number of twenty-four digits being read to him, in 59 seconds he memorized the sound of it, so that he could give the sequence of digits forwards or backwards from selected points-a feat which Mondeux had taken 5 minutes to perform. Numbers of about a hundred digits were similarly memorized by Inaudi in 12 minutes, by Diamandi in 25 minutes, and by Ruickle in under 5 minutes. This power is confined to numbers, and calculators cannot usually recollect a long sequence of letters. Numbers are ever before Inaudi : he thinks of little 
else, he dreams of them, and sometimes even solves problems in his sleep. His memory is excellent for numbers, but normal or subnormal for other things. At the end of a séance he can repeat the questions which have been put to him and his answers, involving hundreds of digits in all. Nor is his memory in such matters limited to a few hours. Once eight days after he had been given a question on a number of twenty-two digits, he was unexpectedly asked about it, and at once repeated the number. He has been repeatedly examined, and we know more of his work than of any of his predecessors, with the possible exception of Bidder.

Most of these calculating prodigies find it difficult or impossible to explain their methods. But we have a few analyses by competent observers of the processes used: notably one by Bidder on his own work; another by Colburn on his work; and others by Muiller and Darboux on the work of Riuckle and Inaudi respectively. That by Bidder is the most complete, and the others are on much the same general lines.

Bidder's account of the processes he had discovered and used is contained in a lecture* given by him in 1856 to the Institution of Civil Engineers. Before describing these processes there are two remarks of a general character which should, I think, be borne in mind when reading his statement. In the first place he gives his methods in their perfected form, and not necessarily in that which he used in boyhood: moreover it is probable that in practice he employed devices to shorten the work which he did not set out in his lecture. In the second place it is certain, in spite of his belief to the contrary, that he, like most of these prodigies, had an exceptionally good memory, which was strengthened by incessant practice. One example will suffice. In 1816, at a performance, a number was read to him backwards: he at once gave it in its normal form. An hour later he was asked if he remembered

* Institution of Civil Engineers, Proceedings, London, 1856, vol. xv, pp. 251-280. An early draft of the leoture is extant in MS.; the variations made in it are interesting, as showing the history of his mental development, but are not sufficiently important to need detailed notice here. 
it: he immediately repeated it correctly. The number was:$2,563,721,987,653,461,598,746,231,905,607,541,128,975,231$.

Of the four fundamental processes, addition and subtraction present no difficulty and are of little interest. The only point to which it seems worth calling attention is that Bidder, in adding three or more numbers together, always added them one at a time, as is illustrated in the examples given below. Rapid mental arithmetic depended, in his opinion, on the arrangement of the work whenever possible, in such a way that only one fact had to be dealt with at a time. This is also noticeable in Inaudi's work.

The multiplication of one number by another was, naturally enough, the earliest problem Bidder came across, and by the time he was six years old he had taught himself the multiplication table up to 10 times 10. He soon had practice in harder sums, for, being a favourite of the village blacksmith, and constantly in the smithy, it became customary for the men sitting round the forge-fire to ask him multiplication sums. From products of numbers of two digits, which he would give without any appreciable pause for thought, he rose to numbers of three and then of four digits. Halfpence rewarded his efforts, and by the time he was eight years old, he could multiply together two numbers each of six digits. In one case he even multiplied together two numbers each of twelve digits, but, he says, "it required much time," and "was a great and distressing effort."

The method that he used is, in principle, the same as that explained in the usual text-books, except that he added his results as he went on. Thus to multiply 397 by 173 he proceeded as follows:-

We have

to this must be added

\begin{tabular}{|c|c|c|c|c|}
\hline 9 & $M$ & , & $\Rightarrow$ & 7 \\
\hline "9 & $\Rightarrow$ & ") & " & 37 \\
\hline " & " & $"$ & $"$ & ענ \\
\hline "y & " & " & ") & " \\
\hline$y$ & נו & ד & " & 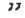 \\
\hline
\end{tabular}

$$
100 \times 397=39700
$$

$$
70 \times 300=21000 \text { making } 60,700 \text {, }
$$$$
70 \times 90=6300 \quad, 67,000 \text {, }
$$$$
70 \times 7=490 \quad, \quad 67,490 \text {, }
$$$$
3 \times 300=900 \quad " 68,390 \text {, }
$$$$
3 \times 90=270 \quad, 68,660 \text {, }
$$$$
3 \times \quad 7=21 \quad \text { " } 68,681 .
$$ 
We shall underrate his rapidity if we allow as much as a second for each of these steps, but even if we take this low standard of his speed of working, he would have given the answer in 7 seconds. By this method he never had at one time more than two numbers to add together, and the factors are arranged so that each of them has only one significant digit: this is the common practice of mental calculators. It will also be observed that here, as always, Bidder worked from left to right: this, though not usually taught in our schools, is the natural and most convenient way. In effect he formed the product of $(100+70+3)$ and $(300+90+7)$, or $(a+b+c)$ and $(d+e+f)$ in the form $a d+a e \ldots+e f$.

The result of a multiplication like that given above was attained so rapidly as to seem instantaneous, and practically gave him the use of a multiplication table up to 1000 by 1000 . On this basis, when dealing with much larger numbers, for instance, when multiplying $965,446,371$ by $843,409,133$, he worked by numbers forming groups of 3 digits, proceeding as if 965,446 , \&c., were digits in a scale whose radix was 1000 : in middle life he would solve a problem like this in about 6 minutes. Such difficulty as he experienced in these multiplications seems to have been rather in recalling the result of the previous step than in making the actual multiplications.

Inaudi also multiplies in this way, but he is content if one of the factors has only one significant digit: he also sometimes makes use of negative quantities : for instance he thinks of $27 \times 729$ as $27(730-1)$; so, too, he thinks of $25 \times 841$ in the form $84100 / 4$ : and in squaring numbers he is accustomed to think of the number in the form $a+b$, choosing $a$ and $b$ of convenient forms, and then to calculate the result in the form $a^{2}+2 a b+b^{2}$.

In multiplying concrete data by a number Bidder worked on similar lines to those explained above in the multiplication of two numbers. Thus to multiply $£ 14$. 15s. $6 \frac{8}{4} d$. by 787 he proceeded thus :

We have

$\mathfrak{E}(787)(14)=£ 11018.0$ s. $0 d$. to which we add (787) (15) shillings $=£ 590.5 s$. 0d. making £11608. 5s. 0d. to which we add (787) (27) farthings =£22. 28. 8t $d$. making £11630.78.8 $d$. 
Division was performed by Bidder much as taught in schoolbooks, except that his power of multiplying large numbers at sight enabled him to guess intelligently and so save unnecessary work. This also is Inaudi's method. A division sum with a remainder presents more difficulty. Bidder was better skilled in dealing with such questions than most of these prodigies, but even in his prime he never solved such problems with the same rapidity as those with no remainder. In public performances difficult questions on division are generally precluded by the rules of the game.

If, in a division sum, Bidder knew that there was no remainder he often proceeded by a system of two-digit terminals. Thus, for example, in dividing (say) 25,696 by 176 , he first argued that the answer must be a number of three digits, and obviously the left-hand digit must be 1 . Next he had noticed that there are only 4 numbers of two digits (namely, 21, 46, 71,96) which when multiplied by 76 give a number which ends in 96 . Hence the answer must be 121 , or 146 , or 171 , or 196 ; and experience enabled him to say without calculation that 121 was too small and 171 too large. Hence the answer must be 146 . If he felt any hesitation he mentally multiplied 146 by 176 (which he said he could do "instantaneously") and thus checked the result. It is noticeable that when Bidder, Colburn, and some other calculating prodigies knew the last two digits of a product of two numbers they also knew, perhaps subconsciously, that the last two digits of the separate numbers were necessarily of certain forms. The theory of these two-digit arrangements has been discussed by Mitchell.

Frequently also in division, Bidder used what I will call a digital process, which a priori would seem far more laborious than the normal method, though in his hands the method was extraordinarily rapid: this method was, I think, peculiar to him. I define the digital of a number as the digit obtained by finding the sum of the digits of the original number, the sum of the digits of this number, and so on, until the sum is less than 10. The digital of a number is the same as the digital of 
the product of the digitals of its factors. Let us apply this in Bidder's way to see if 71 is an exact divisor of 23,141 . The digital of 23,141 is 2. The digital of 71 is 8 . Hence if 71 is a factor the digital of the other factor must be 7 , since 7 times 8 is the only multiple of 8 whose digital is 2 . Now the only number which multiplied by 71 will give 41 as terminal digits is 71. And since the other factor must be one of three digits and its digital must be 7 , this factor (if any) must be 871 . But a cursory glance shows that 871 is too large. Hence 71 is not a factor of 23,141. Bidder found this process far more rapid than testing the matter by dividing by 71 . As another example let us see if 73 is a factor of 23,141. The digital of 23,141 is 2 ; the digital of 73 is 1 ; hence the digital of the other factor (if any) must be 2 . But since the last two digits of the number are 41, the last two digits of this factor (if any) must be 17. And since this factor is a number of three digits and its digital is 2, such a factor, if it exists, must be 317 . This on testing (by multiplying it by 73) is found to be a factor.

When he began to exhibit his powers in public, questions concerning weights and measures were, of course, constantly proposed to him. In solving these he knew by heart many facts which frequently entered into such problems, such as the number of seconds in a year, the number of ounces in a ton, the number of square inches in an acre, the number of pence in a hundred pounds, the elementary rules about the civil and ecclesiastical calendars, and so on. A collection of such data is part of the equipment of all calculating prodigies.

In his exhibitions Bidder was often asked questions concerning square roots and cube roots, and at a later period higher roots. That he could at once give the answer excited unqualified astonishment in an uncritical audience; if, however, the answer is integral, this is a mere sleight of art which anyone can acquire. Without setting out the rules at length, a few examples will illustrate his method.

He was asked to find the square root of 337,561 . It is obvious that the root is a number of three digits. Since the 
given number lies between $500^{2}$ or 250,000 and $600^{2}$ or 360,000 , the left-hand digit of the root must be a 5 . Reflection had shown him that the only numbers of two digits, whose squares end in 61 are $19,31,69,81$, and he was familiar with this fact. Hence the answer was 519 , or 531 , or 569 , or 581 . But he argued that as 581 was nearly in the same ratio to 500 and 600 as 337,561 was to 250,000 and 360,000 , the answer must be 581, a result which he verified by direct multiplication in a couple of seconds. Similarly in extracting the square root of 442,225 , he saw at once that the left-hand digit of the answer was 6 , and since the number ended in 225 the last two digits of the answer were 15 or 35 , or 65 or 85 . The position of 442,225 between $(600)^{2}$ and $(700)^{2}$ indicates that 65 should be taken. Thus the answer is 665 , which he verified, before announcing it. Other calculators have worked out similar rules for the extraction of roots.

For exact cube roots the process is more rapid. For example, asked to extract the cube root of $188,132,517$, Bidder saw at once that the answer was a number of three digits, and since $5^{3}=125$ and $6^{3}=216$, the left-hand digit was 5 . The only number of two digits whose cube ends in 17 is 73 . Hence the answer is 573. Similarly the cube root of $180,362,125$ must be a number of three digits, of which the left-hand digit is a 5 , and the two right-hand digits were either 65 or 85 . To see which of these was required he mentally cubed 560 , and seeing it was near the given number, assumed that 565 was the required answer, which he verified by cubing it. In general a cube root that ends in a 5 is a trifle more difficult to detect at sight by this method than one that ends in some other digit, but since $5^{8}$ must be a factor of such numbers we can divide by that and apply the process to the resulting number. Thus the above number $180,362,125$ is equal to $5^{3} \times 1,442,897$ of which the cube root is at once found to be 5 (113), that is, 565 .

For still higher exact roots the process is even simpler, and for fifth roots it is almost absurdly easy, since the last digit of the number is always the same as the last digit of the root. Thus if the number proposed is less than $10^{10}$ the answer 
consists of a number of two digits. Knowing the fifth powers to $10,20, \ldots 90$ we have, in order to know the first digit of the answer, only to see between which of these powers the number proposed lies, and the last digit being obvious we can give the answer instantly. If the number is higher, but less than $10^{15}$, the answer is a number of three digits, of which the middle digit can be found almost as rapidly as the others. This is rather a trick than a matter of mental calculation.

In his later exhibitions, Bidder was sometimes asked to extract roots, correct to the nearest integer, the exact root involving a fraction. If he suspected this he tested it by "casting out the nines," and if satisfied that the answer was not an integer proceeded tentatively as best he could. Such a question, if the answer is a number of three or more digits, is a severe tax on the powers of a mental calculator, and is usually disallowed in public exhibitions.

Colburn's remarkable feats in factorizing numbers led to similar questions being put to Bidder, and gradually he evolved some rules, but in this branch of mental arithmetic I do not think he ever became proficient. Of course a factor which is a power of 2 or of 5 can be obtained at once, and powers of 3 can be obtained almost as rapidly. For factors near the square root of a number he always tried the usual method of expressing the number in the form $a^{2}-b^{2}$, in which case the factors are obvious. For other factors he tried the digital method already described.

Bidder was successful in giving almost instantaneously the answers to questions about compound interest and annuities : this was peculiar to him, but his method is quite simple, and may be illustrated by his determination of the compound interest on $£ 100$ at 5 per cent. for 14 years. He argued that the simple interest amounted to $f(14)(5)$, i.e. to $£ 70$. At the end of the first year the capital was increased by $\mathfrak{f 5}$, the annual interest on this was $5 s$. or one crown, and this ran for 13 years, at the end of the second year another $\mathfrak{f 5}$ was due, and the $5 s$. interest on this ran for $\mathbf{1 2}$ years. Continuing this argument he had to add to the $£ 70$ a sum of $(13+12+\ldots+1)$ crowns, i.e. (13/2) (14) (5) shillings, i.e. £22. 15s. 0ds, which, added to the 
$£ 70$ before mentioned, made $£ 92.15 s .0 d$. Next the $5 s$, due at the end of the second year (as interest on the $£ 5$ due at the end of the first year) produced in the same way an annual interest of $3 d$. All these three-pences amount to $(12 / 3)(13 / 2)(14)(3)$ pence, i.e. $£ 4.11 s$. $0 d$. which, added to the previous sum of £92. 15s. Od., made £97. 6s. 0d. To this we have similarly to add $(11 / 4)(12 / 3)(13 / 2)(14)(3 / 20)$ pence, i.e. $12 s .6 d$., which made a total of $£ 97.18 \mathrm{~s} .6 \mathrm{~d}$. To this again we have to add $(1.0 / 5)(11 / 4)(12 / 3)(13 / 2)(14)(3 / 400)$ pence, i.e. 1s. 3d., which made a total of $£ 97$. 19s. $9 d$. To this again we have to add $(9 / 6)(10 / 5)(11 / 4)(12 / 3)(13 / 2)(14)(3 / 8000)$ pence, i.e. 1d., which made a total of $\mathfrak{x} 97.19 \mathrm{~s}$. $10 \mathrm{~d}$. The remaining sum to be added cannot amount to a farthing, so he at once gave the

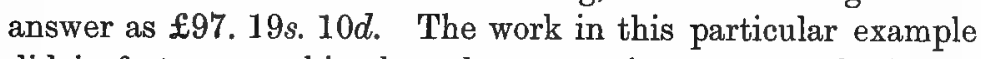
did in fact occupy him less than one minute-a much shorter time than most mathematicians would take to work it by aid of a table of logarithms. It will be noticed that in the course of his analysis he summed various series.

In the ordinary notation, the sum at compound interest amounts to $\mathfrak{f}(1 \cdot 05)^{14} \times 100$. If we denote $£ 100$ by $P$ and $\cdot 05$ by $r$, this is equal to $P(1+r)^{14}$ or $P\left(1+14 r+91 r^{2}+\ldots\right)$, which, as $r$ is small, is rapidly convergent. Bidder in effect arrived by reasoning at the successive terms of the series, and rejected the later terms as soon as they were sufficiently small.

In the course of this lecture Bidder remarked that if his ability to recollect results had been equal to his other intellectual powers he could easily have calculated logarithms. A few weeks later he attacked this problem, and devised a mental method of obtaining the values of logarithms to seven or eight places of decimals. He asked a friend to test his accuracy, and in answer to questions gave successively the logarithms of $71,97,659,877,1297,8963,9973,115249$, $175349,290011,350107,229847,369353$, to eight places of decimals; taking from thirty seconds to four minutes to make the various calculations. All these numbers are primes. The greater part of the answers were correct, but in a few cases there was an error, though generally of only one digit: such

B. $\mathbf{R}$. 
mistakes were at once corrected on his being told that his result was wrong. This remarkable performance took place when Bidder was over 50 .

His method of calculating logarithms is set out in a paper* by W. Pole. It was, of course, only necessary for him to deal with prime numbers, and Bidder began by memorizing the logarithms of all primes less than 100. For a prime higher than this he took a composite number as near it as he could, and calculated the approximate addition which would have to be added to the logarithm: his rules for effecting this addition are set out by Pole, and, ingenious though they are, need not detain us here. They rest on the theorems that, to the number of places of decimals quoted, if $\log n$ is $p$, then $\log \left(n+n / 10^{2}\right)$ is $p+\log 1 \cdot 01$, i.e. is $p+0.0043214, \log \left(n+n / 10^{3}\right)$ is $p+0.00043407, \log \left(n+n / 10^{4}\right)$ is $p+0.0000434, \log \left(n+n / 10^{5}\right)$ is $p+0.0000043$, and so on.

The last two methods, dealing with compound interest and logarithms, are peculiar to Bidder, and show real mathematical skill. For the other problems mentioned his methods are much the same in principle as those used by other calculators, though details vary. Bidder, however, has set them out so clearly that I need not discuss further the methods generally used.

A curious question has been raised as to whether a law for the rapidity of the mental work of these prodigies can be found. Personally I do not think we have sufficient data to enable us to draw any conclusion, but I mention briefly the opinions of others. We shall do well to confine ourselves to the simplest case, that of the multiplication of a number of $n$ digits by another number of $n$ digits. Bidder stated that in solving such a problem he believed that the strain on his mind (which he assumed to be proportional to the time taken in answering the question) varied as $n^{4}$, but in fact it seems in his case according to this time test to have varied approximately as $n^{5}$. In 1855 he worked at least half as quickly again as in 1819, but the law of rapidity for different values of $n$ is said to have been

* Institution of Civil Engineers, Proceedings, London, 1890-1891, vol. arr, p. 250 . 
about the same. In Dase's case, if the time occupied is proportional to $n^{x}$, we must have $x$ less than 3. From this, some have inferred that probably Dase's methods were different in character to those used by Bidder, and it is suggested that the results tend to imply that Dase visualized recorded numerals, working in much the same way as with pencil and paper, while Bidder made no use of symbols, and recorded successive results verbally in a sort of cinematograph way; but it would seem that we shall need more detailed observations before we can frame a theory on this subject.

The cases of calculating prodigies here mentioned, and as far as I know the few others of which records exist, do not differ in kind. In most of them the calculators were uneducated and self-taught. Blessed with excellent memories for numbers, self-confident, stimulated by the astonishment their performances excited, the odd coppers thus put in their pockets and the praise of their neighbours, they pondered incessantly on numbers and their properties; discovered (or in a few cases were taught) the fundamental arithmetical processes, applied them to problems of ever increasing difficulty, and soon acquired a stock of information which shortened their work. Probably constant practice and undivided devotion to mental calculation are essential to the maintenance of the power, and this may explain why a general education has so often proved destructive to it. The performances of these calculators are remarkable, but, in the light of Bidder's analysis, are not more than might be expected occasionally from lads of exceptional abilities. 


\section{CHAPTER XIV.}

\section{CRYPTOGRAPHS AND CIPHERS.}

The art of constructing cryptographs or ciphers-intelligible to those who know the key and unintelligible to others-has been studied for centuries. Their usefulness on certain occasions, especially in time of war, is obvious, while their right interpretation may be a matter of great importance to those from whom the key is concealed. But the romance connected with the subject, the not uncommon desire to discover a secret, and the implied challenge to the ingenuity of all from whom it is hidden, have attracted to the subject the attention of many to whom its utility is a matter of indifference.

Among the best known of the older authorities on the subject are J. Tritheim of Spanheim, G. Porta of Naples, G. Cardan, J. F. Niceron, J. Wilkins, and E. A. Poe. More modern writers are J. E. Bailey in the Encyclopaedia Britannica, E. B. von Wostrowitz of Vienna, 1881, F. Delastelle of Paris, 1902, and J. L. Kluiber of Tübingen, 1809. My knowledge, however, is largely the result of casual reading, and I prefer to discuss the subject as it has presented itself to me, with no attempt to make it historically complete.

Most writers use the words cryptograph and cipher as synonymous. I employ them, however, with different meanings, which I proceed to define.

A cryptograph may be defined as a manner of writing in which the letters or symbols employed are used in their normal sense, but are so arranged that the communication is intelligible 
only to those possessing the key: the word is also sometimes used to denote the communication made. A simple example is a communication in which every word is spelt backwards. Thus :

\section{ymene deveileb ot eb gniriter troper noitisop no ssorc daor.}

A cipher may be defined as a manner of writing by characters arbitrarily invented or by an arbitrary use of letters, words, or characters in other than their ordinary sense, intelligible only to those possessing the key: the word is also sometimes used to denote the communication made. A simple example is when each letter is replaced by the one that immediately follows it in the natural order of the alphabet, $a$ being replaced by $b, b$ by $c$, and so on, and finally $z$ by $a$. In this cipher the above message would read:

\section{fofnz cfnijfwfe up of sfujsjoh sfqpsu qptjujpo po dsptt spbe.}

In both cryptographs and ciphers the essential feature is that the communication may be freely given to all the world though it is unintelligible save to those who possess the key. The key must not be accessible to anyone, and if possible it should be known only to those using the cryptograph or cipher. The art of constructing a cryptograph lies in the concealment of the proper order of the essential letters or words: the art of constructing a cipher lies in concealing what letters or words are represented by the symbols used.

In an actual communication cipher symbols may be arranged cryptographically, and thus further hinder a reading of the message. Thus the message given above might be put in a cryptographic cipher as

znfof efwfjmfc pu fc hojsjufs uspqfs opjujtpq op ttpsd ebps.

If the message were written in a foreign language it would further diminish the chance of it being read by a stranger through whose hands it passed. But I may confine myself to messages in English, and for the present to simple cryptographs and ciphers.

A communication in cryptograph or cipher must be in 
writing or in some permanent form. Thus to make small muscular movements-such, ex. gr., as talking on the fingers, or breathing long and short in the Morse dot and dash system, or making use of pre-arranged signs by a fan or stick, or flashing signals by light-do not here concern us.

The mere fact that the message is concealed or secretly conveyed does not make it a cryptograph or cipher. The majority of stories dealing with secret communications are concerned with the artfulness with which the message is concealed or conveyed and have nothing to do with cryptographs or ciphers. Many of the ancient instances of secret communication are of this type. Illustrations are to be found in messages conveyed by pigeons, or wrapped round arrows shot over the head of a foe, or written on the paper wrapping of a cigarette, or by the use of ink which becomes visible only when the recipient treats the paper on which it is written by some chemical or physical process.

Again, a communication in a foreign language or in any recognized notation like shorthand is not an instance of a cipher. A letter in Chinese or Polish or Russian might be often used for conveying a secret message from one part of England to another, but it fails to fulfil our test that if published to all the world it would be concealed, unless submitted to some special investigation. On the other hand, in practice, foreign languages or systems of shorthand which are but little known may serve to conceal a communication better than an easy cipher, for in the last case the key may be found with but little trouble, while in the other cases, though the key may be accessible, it is probable that there are only a few who know where to look for it.

Cryptographs. I proceed to enumerate some of the better known types of cryptographs. There are at least three distinct types. The types are not exclusive, and any particular cryptograph may comprise the distinctive feature of two or all the types.

First Type of Cryptographs (Transposition Type). A cryptograph of the first type is one in which the successive letters 
or words of the message are re-arranged in some pre-determined manner.

One of the most obvious cryptographs of this type is to write each word or the message itself backwards. Here is an instance in which the whole message is written backwards:

tsop yb tnes tnemeerga fo seniltuo smret ruo tpecca yeht.

It is unnecessary to indicate the division into words by leaving spaces between them, and we might introduce capitals or make a pretence of other words, as thus:

Ts opybtne stne meer gafos eniltu Osmret ruot peccaye ht.

A recipient who was thus mis-led would be very careless. Preferably, according to modern practice, we should write the message in groups of five letters each: the advantage of such a division being that the number of such groups can be also communicated, and the casual omission of letters thus detected.

Systems of this kind which depend on altering the places of letters or lines in some pre-arranged manner have always been common. One example is where the letters which make up the communication are written vertically up or down. 'Thus the message: The pestilence continues to increase, might be eiotnlit written in 8 columns as shown in the margin, sntioeth and then sent in five letter groups as eiotn acsncnse litsn, etc. If before reading off the message ereuecep. the 8 columns were interchanged according to some prearranged scheme the cryptograph would be greatly improved, and this is said to be a method used in the German Army. This cryptograph might be further obscured by writing the 32 letters according to the Route Method described below.

Another method is to write successively the 1st, 18th, 35th letters of the original message, and then the $2 \mathrm{nd}, 19 \mathrm{th}, 36 \mathrm{th}$ letters, and so on. If, however, we know the clue number, say c, it is easy enough to read the communication. For if it divides into the number of letters $n$ times with a remainder $r$ it suffices to re-write the message in lines putting $n+1$ letters in each of the first $r$ lines, and $n$ letters in each of the last $c-r$ lines, 
and then the communication can be read by reading the columns downwards. For instance, if the following communication, containing 270 letters, were received: Ahtze ipqhg esoae ouazs esewa eqtmu sfdtb enzce sjteo ttqiz yczht zjioa rhqet tjrfe sftnz mroom ohyea rziaq neorn breot lennk aerwi zesju asjod ezwjz zszjb rritt jnfjl weuzr oqyfo htqay eizsl eopji dihal oalhp eplcrh eanaz srvli imosi adygt pekij scerq vvjqj qajqn yjint kaehs bhsnb goaot qetqe uuesa yqurn tpebq stzam ztqrj, and the clue number were 17 we should put 16 letters in each of the first 15 lines and 15 letters in each of the last 2 lines. The communication could then be discovered by reading the columns downwards : the letters $j, q$ and $z$ marking the ends of words.

A better cryptograph of this kind may be made by arranging the letters cyclically, and agreeing that the communication is to be made by selected letters, as, for instance, every seventh, second, seventh, second, and so on. Thus if the communication were Ammunition too low to allow of a sortie, which consists of 32 letters, the successive significant letters would come in the order $7,9,16,18,25,27,2,4,13,15,24,28,5,8,20,22,1,6$, $21,26,11,14,32,10,31,12,17,23,3,29,30,19$-the numbers being selected as in the decimation problem given above at the end of chapter I, and being struck out from the 32 cycle as soon as they are determined. The above communication would then read Ttrio oalmo laoon msueo awotn lioti fw. This is a good method, but it is troublesome to use, and for that reason is not to be recommended.

In another cryptograph of this type, known as the Route Method, the words are left unaltered, but are re-arranged in a

\begin{tabular}{|c|c|c|c|c|c|c|}
\hline 11 & 8 & 13 & 2 & 15 & 4 & $w$ \\
\hline$x$ & 1 & 10 & 17 & 6 & $y$ & $z$ \\
\hline 9 & 12 & 7 & 14 & 3 & 16 & 5 \\
\hline
\end{tabular}

pre-determined manner. Thus, to take a very simple example, the words might be written in tabular form in the order shown 
in the diagram, certain spaces being filled with dummy words $x, y, z, \ldots$, and the message being sent in the order $11,8,13,2$, $15,4, w, x, 1, \ldots$ This method was used successfully by the Federals in the American Civil War, 1861-1865, equivalents for proper names being used. It is easy to work, but the key would soon be discovered by modern experts.

A double cryptograph is said to have been used by the Nihilists in Russia from 1890-1900. Such double transpositions are always awkward, and mistakes, which would make the message unintelligible, may be easily introduced, but if time is of little importance, and the message is unlikely to fall into the hands of any but ordinary officials, the concealment is fairly effective, though a trained specialist who had several messages in it could work out the key.

Second Type of Cryptographs. A cryptograph of the second type is one in which the message is expressed in ordinary writing, but in it are introduced a number of dummies or nonsignificant letters or digits thus concealing which of the letters are relevant.

One way of picking out those letters which are relevant is by the use of a perforated card of the shape of (say) a sheet of note-paper, which when put over such a sheet permits only such letters as are on certain portions of it to be visible. Such a card is known as a grille. An example of a grille with four openings is figured below. A communication made in this

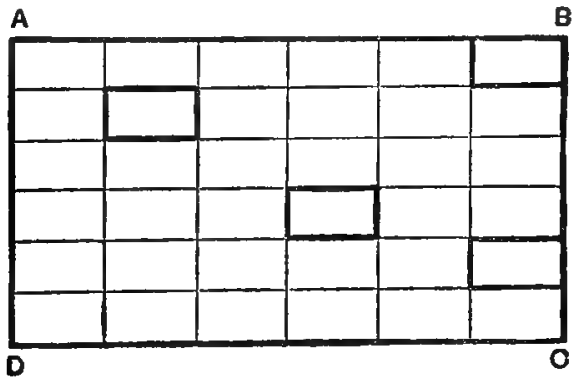

way may be easily concealed from anyone who does not possess a card of the same pattern. If the recipient possesses such 
a card he has only to apply it in order to read the message. This method was used by Richelieu.

The use of the grille may be rendered less easy to detect if it be used successively in different positions, for instance, with the edges $A B$ and $C D$ successively put along the top of the paper containing the message. Below, for instance, is a message which, with the aid of the grille figured above, is at once intelligible. On applying the grille to it with the line $A B$ along the top $H K$ we get the first half of the communication, namely, 1000 rifles se. On applying the grille with the

\begin{tabular}{|c|c|c|c|c|c|}
\hline 981 & 264 & 070 & 523 & 479 & 100 \\
\hline$N T T$ & $O R I$ & SON & SON & $A H Y$ & $D T C$ \\
\hline$B F S$ & $P U M$ & $O L T$ & $K K F E$ & $L J O$ & $E G X$ \\
\hline$\triangle E U$ & $Q J T$ & $E G O$ & $F L E$ & $H V E$ & $W L A$ \\
\hline$F M L$ & $A E S$ & $R E M$ & $R E M$ & $O D A$ & $S S E$ \\
\hline$Y Z Z$ & $E P D$ & $Q J C$ & $E K S$ & TIM & $O E F$ \\
\hline
\end{tabular}

line $C D$ along the top $H K$ we get the rest of the message, namely, nt to $L$ to-day. The other spaces in the paper are filled with non-significant letters or numerals in any way we please. Of course any one using such a grille would not divide the sheet of paper on which the communication was written into cells, but in the figure $I$ have done so in order to render the illustration clearer.

We can aroid the awkward expedient of having to use a perforated card, which may fall into undesired hands, by introducing a certain pre-arranged number of dummies or non-significant letters or symbols between those which make up the message. For instance, we might arrange that (say) only every alternate second and third letter shall be relevant. Thus the first, third, sixth, eighth, eleventh, \&c., letters are those that make up the message. Such a communication would be two and a half times as long as the message, and this might 
be a great disadvantage if time in sending the message was of importance.

Another method, essentially the same as the grille method, is to arrange that every $n$th word shall give the message, the other words being non-significant, though of course inserted as far as possible so as to make the complete communication run as a whole. But the difficulty of composing a document of this kind and its great length render it unsuitable for any purpose except an occasional communication composed at leisure and sent in writing. This method is said to have been used by the Earl of Argyle when plotting against James II.

Third Type of Cryptographs. A kind of secret writing which may perhaps be considered to constitute a third type of cryptograph is a communication on paper which is legible only when the paper is folded in a particular way. An example is a message written across the edges of a strip of paper wrapped spiral-wise round a stick called a scytale. When the paper is unwound and taken off the stick the letters appear broken, and may seem to consist of arbitrary signs, but by wrapping the paper round a similar stick the message can be again read. This system is said to have been used by the Lacedemonians. The concealment can never have been effectual against an intelligent reader who got possession of the paper. As another illustration take the appended communication which

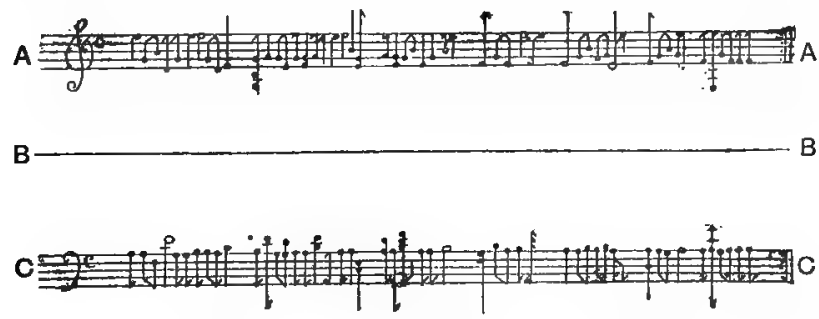

is said to have been given to the Young Pretender during his wanderings after Culloden. If it be creased along the lines $B B$ and $C C$ ( $C C$ being along the second line of the second score), and then folded over, with $B$ inside, so that the crease $C$ lies 
over the line $A$ (which is the second line of the first score) thus leaving only the top and bottom of the piece of paper visible, it will be found to read Conceal yourself, your foes look for you. I have seen what purports to be the original, but of the truth of the anecdote I know nothing, and the desirability of concealing himself must have been so patent that it was hardly necessary to communicate it by a cryptograph.

Ciphers. I proceed next to some of the more common types of ciphers. It is immaterial whether we employ special characters to denote the various letters; or whether we use the letters in a non-natural sense, such as the letter $z$ for $a$, the letter $y$ for $b$, and so on. In the former case it is desirable to use symbols, for instance, musical notes, which are not likely to attract special notice. Geometrical figures have also been used for the same purpose. It is not even necessary to employ written signs. Natural objects have often been used, as in a necklace of beads, or a bouquet of flowers, where the different shaped or coloured beads or different flowers stand for different letters or words. An even more subtle form of disguising the cipher is to make the different distances between consecutive knots or beads indicate the different letters. Of all such systems we may say that a careful scrutiny shows that different symbols are being used, and as soon as the various symbols are distinguished one from the other no additional complication is introduced, while for practical purposes they give more trouble to the sender and the recipient than those written in symbols in current use. Accordingly I confine myself to ciphers written by the use of the current letters and numerals. There are four types of ciphers.

First Type of Ciphers. Simple Substitution Alphabets. A cipher of the first type is one in which the same letter or word is always represented by the same symbol, and this symbol always represents the same letter or word.

Perhaps the simplest illustration of a cipher of this type is to employ one language, written as far as practical in the alphabet of another language. It is said that during the Indian Mutiny messages in English, but written in Greek 
characters, were used freely, and successfully baffled the ingenuity of the enemy, into whose hands they fell.

A common cipher of this type is made by using the actual letters of the alphabet, but in a non-natural sense as indicating other letters. Thus we may use each letter to represent the one immediately following it in the natural order of the alphabetthe letters being supposed to be cyclically arranged- $a$ standing for $b$ wherever it occurs, $b$ standing for $c$, and so on, and finally $z$ standing for $a$ : this scheme is said to have been used by the Carthaginians and Romans.

More generally we may write the letters of the alphabet in a line, and under them re-write the letters in any order we like. For instance

$a b c d e f g h i j k l m n$ opqrstuvwxyz olkmazs qxeufyrthcwbvnidgjp

In such a scheme, we must in our communication replace $a$ by $o, b$ by $l$, etc. The recipient will prepare a key by rearranging the letters in the second line in their natural order and placing under them the corresponding letter in the first line. Then whenever $a$ comes in the message he receives he will replace it by an $e$; similarly he will replace $b$ by $s$, and so on.

A cipher of this kind is not uncommonly used in military signalling, the order of the letters being given by the use of a key word. Ciphers of this class were employed by the British forces in the Sudan and South African campaigns. If, for instance, Pretoria is chosen as the key word, we write the letters in this order, striking out any which occur more than once, and continue with the unused letters of the alphabet in their natural order, writing the whole in two lines thus:

$$
\begin{aligned}
& p r e t o i a b c d f g h \\
& z y x w v u s q n m l k j
\end{aligned}
$$

Then in using the cipher $p$ is replaced by $z$ and vice versa, $r$ by $y$, and so on. A long message in such a cipher would be easily discoverable, but it is rapidly composed by the sender and read by the receiver, and for some purposes may be useful, 
especially if the discovery of the purport of the message is, after a few hours, immaterial.

The key to ciphers of this type may usually be found by using tables of the normal frequency with which letters may be expected to occur. Such tables, and other characteristic features of the English, French, German, Italian, Dutch, Latin, and Greek languages, were given by D. A. Conrad in 1742*. His results have since been revised, and extended to Russian, Spanish, and other tongues. In English the percentage scale of frequency of the letters is approximately as follows:- $e, 12 \cdot 0 ; t, 9 \cdot 4 ; a, 7 \cdot 8$; $0,7 \cdot 5 ; i, 7 \cdot 4 ; n, 7 \cdot 3 ; s, 6.8 ; r, 5.9 ; h, 5 \cdot 7 ; d, 3.9 ; l, 3.6 ; u, 3.0$; $c, 2 \cdot 8 ; m, 2 \cdot 7 ; f, 2 \cdot 5 ; p, 1.9 ; g, 1 \cdot 8 ; y, 1 \cdot 8 ; b, 1 \cdot 7 ; w, 1 \cdot 7 ; v, 1 \cdot 1$; $k, 06 ; j, 0.3 ; q, 0.3 ; x, 0.3 ; z, 0.2$. The order of frequency for combinations of two letters is th, he, in, an, on, re, ti, er, it, nt, $e s, t o, s t$; of three letters is the, ion, \&c., \&c.; of four letters is tion, that, \&c., \&c.; and of double letters is $t t, s s$, \&c., \&c. Other peculiarities, such as that $h, l, m, n, v$, and $y$, when at the beginning of a word, must be followed by a vowel, that $q$ must be followed by $u$ and another vowel, have been classified and are important. I need not go here into further details. Unless, however, the message runs to 400 words or more, we cannot reasonably expect to find the scale of frequency the same as in Conrad's Table.

In ciphers of this class it is especially important to avoid showing the division into words, for a long word may easily betray the secret. For instance, if the decipherer has reason to suspect that the message related to something connected with Birmingham, and he found that a particular word of ten letters had its second and fifth letters alike, as also its fourth and tenth letters, he would naturally see how the key would work if the word represented Birmingham, and on this hypothesis would at once know the letters represented by eight symbols. With reasonable luck this should suffice to enable him to tell if the hypothesis was tenable. To avoid this risk it

* Gentleman's Magazine, 1742, vol. xIr, pp. 133-135, 185-186, 241-242, 473-475. See also the Collected Works of 2. A. Poe in 4 volumes, vol. I, p. 30 et seq. 
is usual to send the cipher in groups of five letters, and, before putting it into cipher, to separate the words in the message by letters like $j, q, x, z$.

Ciphers of this type suggest themselves naturally to those approaching the subject for the first time, and are commonly made by merely shifting the letters a certain number of places forward. If this is done we may decrease the risk of detection by altering the amount of shifting at short (and preferably irregular) intervals. Thus it may be agreed that if initially we shift every letter one place forward then whenever we come to the letter (say) $n$ we shall shift every letter one more place forward. In this way the cipher changes continually, and is essentially changed to one of the third class; but even with this improvement it is probable that an expert would decode a fairly long message without much difficulty.

We can have ciphers for numerals as well as for letters: such ciphers are common in many shops. Any word or sentence containing ten different letters will answer the purpose. Thus, an old tradesman of my acquaintance used the excellent precept Be just O Man-the first letter representing 1, the second 2, and so on. In this cipher the price 10/6 would be marked $b n / t$. This is an instance of a cipher of the first type.

Second Type of Ciphers. A cipher of the second type is one in which the same letter or word is, in some or all cases, represented by more than one symbol, and this symbol always represents the same letter or word. Such ciphers were uncommon before the Renaissance, but the fact that to those who held the key they were not more difficult to write or read than ciphers of the first type, while the key was not so easily discovered, led to their common adoption in the seventeenth century.

A simple instance of such a cipher is given by the use of numerals to denote the letters of the alphabet. Thus $a$ may be represented by 11 or by 37 or by $63, b$ by 12 or by 38 or by 64 , and so on, and finally $z$ by 36 or by 62 or by 88 , while we can use 89 or 90 to signify the end of a word and the numbers 91 to 99 to denote words or sentences which constantly occur. Of course in practice no one would employ the 
numbers in an order like this, which suggests their meaning, but it will serve to illustrate the principle.

The cipher can be improved by introducing after every (say) eleventh digit a non-significant digit. If this is done the recipient of the message must erase every twelfth digit before he begins to read the message. With this addition the difficulty of discovering the key is considerably increased.

The same principle is sometimes applied with letters instead of numbers. For instance, if we take a word (say) of $n$ letters, preferably all different, and construct a table as shown below of $n^{2}$ cells, each cell is defined by two letters of the key word. Thus, if we choose the word smoking-cap we shall have 100

\begin{tabular}{|c|c|c|c|c|c|c|c|c|c|c|}
\hline & $\boldsymbol{S}$ & $M$ & 0 & $\boldsymbol{K}$ & $I$ & $N$ & $G$ & $C$ & $\boldsymbol{A}$ & $P$ \\
\hline $\boldsymbol{S}$ & B & b & 0 & $\mathbf{d}$ & e & f & g & h & $\mathbf{i}$ & $\mathbf{j}$ \\
\hline$M$ & $\mathbf{k}$ & 1 & $\mathbf{m}$ & $\mathbf{n}$ & 0 & $\mathbf{p}$ & $\mathbf{q}$ & $\boldsymbol{r}$ & 8 & $\mathbf{t}$ \\
\hline 0 & $\mathbf{u}$ & $\nabla$ & $\mathbf{T}$ & $\mathbf{x}$ & $\mathbf{y}$ & $\mathbf{z}$ & $\mathbf{a}$ & b & 0 & $d$ \\
\hline $\bar{K}$ & e & 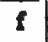 & B & $\bar{b}$ & i & $\mathbf{j}$ & $\mathbf{k}$ & 1 & $\mathbf{m}$ & $\mathbf{n}$ \\
\hline$I$ & 0 & $\mathbf{p}$ & $\mathbf{q}$ & $\mathbf{r}$ & $\mathbf{B}$ & $t$ & $\mathbf{u}$ & $\mathbf{v}$ & $w$ & $x$ \\
\hline$N$ & $y$ & $\mathrm{z}$ & 8 & b & 0 & $\mathrm{~d}$ & 0 & $f$ & $\mathbf{g}$ & $\mathbf{b}$ \\
\hline$G$ & $\mathbf{i}$ & $\mathrm{j}$ & $\mathrm{k}$ & 1 & $\mathrm{~m}$ & $\mathbf{n}$ & 0 & $\mathbf{p}$ & $q$ & $\mathbf{r}$ \\
\hline$c$ & 8 & $t$ & $\mathbf{u}$ & $\nabla$ & $\mathbf{W}$ & $\mathbf{x}$ & 8 & z & & \\
\hline$A$ & & & & & & & & & & \\
\hline $\boldsymbol{P}$ & & & & & & & & & & \\
\hline
\end{tabular}

cells, and each cell is determined uniquely by the two letters denoting its row and column. If we fill these cells in order with the letters of the alphabet we shall have a system similar to that explained above, where $a$ will be denoted by ss or og or no, and so for the other letters. The last 22 cells may be used to denote the first 22 letters of the alphabet, or better, three or four of them may be used after the end of a word to show that it is ended, and the rest may be used to denote words or sentences which are likely to occur frequently. The statement in cipher however is twice as long as when it is in clear.

Like the similar cipher with numbers this can be improved 
by introducing after every $m$ th letter any single letter which it is agreed shall be non-significant. To decipher a communication so written it is necessary to know the clue word and the clue number.

Here for instance is a communication written in the above cipher with the clue word smoking-cap, and with 7 as the clue number: ngmks igrio icpss amcks cakqi gnass nxmig poasu iamno cmpam inscn ogcpn cisyi leskam sssgn nncae kknoo mkhsc pcmsc bgpng siaws sgigg ndiic a. In this sentence the letters denoting the 79th, $80 \mathrm{th}, 81 \mathrm{st}$, and $82 \mathrm{nd}$ cells have been used to denote the end of a word, and no use has been made of the last 18 cells.

Another cipher of this type is made as follows. The sender and recipient of the message furnish themselves with identical copies of some book. In the cipher only numerals are used, and these numerals indicate the locality of the letters in the book. For example, the first letter in the communication might be indicated by 79-8-5, meaning that it is the 5 th letter in the 8th line of the 79th page. But though secrecy might be secured, it would be very tedious to prepare or decode a message, and the method is not as safe as some of those described below.

Another cipher of this type is obtained by the sender and receiver agreeing on some common book of reference and further on a number which, if desired, may be communicated as part of the message. To employ this method the page of the book indicated by the given number must be used. The first letter in it is taken to signify $a$, the next $b$, and so onany letter which occurs a second time or more frequently being neglected. It may be also arranged that after $n$ letters of the message have been ciphered, the next $n$ letters shall be written in a similar cipher taken from the $p$ th following page of the book, and so on. Thus the possession of the code-book would be of little use to anyone who did not also know the numbers employed. It is so easy to conceal the clue number that with ordinary prudence it would be almost impossible for an unauthorized person to discover a message sent in this cipher. 
The clue number may be communicated indirectly in many ways. For instance, it may be arranged that the number to be used shall be the number sent, plus (say) $q$, or that the number to be used shall be an agreed multiple of the number actually sent.

Third Type of Ciphers. Complex Shifting Alphabets. A cipher of the third type is one in which the same symbol represents sometimes one letter or word and sometimes another.

A simple example, known as Gronfeld's Method, is the employment of pre-arranged numbers in shifting forward the letters that make the communication. For instance, if we agree on the key number 6814 , then the first letter in the communication is replaced by the sixth letter which follows it in the natural order of the alphabet: for instance, if it were an $a$ it would be replaced by $g$. The next letter is replaced by the eighth letter which follows it in the natural order of the alphabet: for instance, if it were an $a$ it would be replaced by $i$. The next letter is replaced by the first after it; the next by the fourth after it; the next by the sixth; and so on to the end of the message. Of course to read the message the recipient would reverse the process. If the letters of the alphabet are written at uniform intervals along a ruler, and another ruler similarly marked with the digits is made to slide along it, the letter corresponding to the shifting of any given number of places can be read at once. Here is such a message :-Cisvg vumya vijnp vgzsi ybpjp woiy. Such ciphers are easy to make and read by those who have the key. But in recent years their construction has been subjected to critical analysis, and experts now can generally obtain the key number if the message contains 80 or 100 words; an example of the way by which this is done is given below. It would be undesirable to allow the division into words to appear in the message, and either the words must be run on continuously, or preferably the less common letters $j, q, z$ may be used to mark the division of words and the message then written in five letter groups.

It is most important to conceal the number of digits in the 
key number. The difficulty of discovering the key number is increased if after every (say) $m$ th letter (or word) a non-significant letter is inserted. I suggest this as an improvement in the cipher.

Here for instance is a communication written in this cipher with the clue numbers 4276 and 7: atpzn hvaxu shiep xafwg hzniy prpsi kbdkz yygkq prgez uytlk obldi febzm xlpog quyit cmgxk ckuex vsqka ziagg sigay tnvvs styvu aslyw gjuzm csfct qbpwj vaepf xhibw pxiul txlav vtqzo xwkvt uvvfh cqbxn pvism phamq tuwxj ykeev ltif. The recipient would begin by striking out every eighth letter. He would then shift, back every letter $4,2,7,6,4,2$, \&c., places respectively, and in reading it, would leave out the letters $j, q$, and $z$ as only marking the ends of words.

With these modifications, this is an excellent cipher, and it has the additional merit of not materially lengthening the message. It can be rendered still more difficult by arranging that either or both the clue numbers shall be changed according to some definite scheme, and it may be further agreed that they shall change automatically every day or week.

A similar system, now known as the St Cyr Method, was proposed by Wilkins*. He took a key word, such as prudentia, and constructed as many alphabets as there were letters in it, each alphabet being arranged cyclically and beginning respectively with the letters $p, r, u, d, e, n, t, i$, and $a$. He thus got a table like the following, giving nine possible letters which might stand for any letter of the alphabet. Using this we may vary the cipher in successive words or letters of the communication. Thus the message The prisoners have mutinied and seized the railway station would, according as the cipher changes in successive words or letters, read as Hwt fhziedvhi bupy pxwmqmhy erh ervmrq max zirteig station or as Hyy svvlwnthm lehx uuhzgmiq tvd gvcciq mqe frcoanr atpkcrr.

The name by which the method is known is derived from the fact that it was taught at St Cyr under Napoleon. This system is said to have been widely employed by both armies in the Franco-German war in 1870-1871. The construction of military ciphers must be so simple that messages can be rapidly

- Mercury, by J. Wilkins, London, 1641, pp. 59, 60. 
enciphered and decoded by non-experts: the St Cyr code fulfils this requirement.

\begin{tabular}{|c|c|c|c|c|c|c|c|c|c|c|c|c|c|c|c|c|c|c|c|c|c|c|c|c|}
\hline$a$ & $b$ & c & $d$ & $\mathrm{e}$ & $f$ & $\mathrm{~g}$ & h & i & $\mathrm{k}$ & 1 & $\mathbf{m}$ & $\mathbf{n}$ & 0 & $\mathbf{p}$ & $q$ & $\mathbf{r}$ & $\mathbf{8}$ & $t$ & $\mathbf{v}$ & $v$ & $\mathbf{w}$ & $\mathbf{I}$ & $y$ & $z$ \\
\hline$p$ & $\mathbf{q}$ & $\mathbf{r}$ & $\mathrm{s}$ & $t$ & $\mathbf{u}$ & $\mathbf{v}$ & $\mathbf{w}$ & $\mathbf{x}$ & $y$ & $\mathbf{z}$ & a & $\mathrm{b}$ & c & $d$ & $\theta$ & $i$ & g & h & 1 & k & 1 & $\mathrm{~m}$ & $\mathbf{n}$ & 0 \\
\hline$r$ & s & $\mathbf{t}$ & $\mathbf{u}$ & $v$ & $w$ & $x$ & $\mathbf{y}$ & 2 & a & b & c & $\mathbf{d}$ & e & $?$ & $\mathrm{~g}$ & $\mathbf{h}$ & i & $\mathbf{k}$ & 1 & $\mathrm{~m}$ & $\mathbf{n}$ & 0 & $\mathbf{p}$ & $q$ \\
\hline$u$ & $v$ & $\mathrm{w}$ & $x$ & $y$ & $\mathbf{z}$ & $\mathbf{a}$ & b & 0 & d & e & f & $\mathrm{g}$ & $\mathbf{h}$ & i & $\mathrm{k}$ & 1 & $\mathrm{~m}$ & $\mathbf{n}$ & 0 & $\mathbf{p}$ & $\mathbf{q}$ & $\mathbf{r}$ & 8 & $t$ \\
\hline$d$ & e & $\mathbf{f}$ & $\mathbf{g}$ & $\mathrm{h}$ & $\mathbf{i}$ & $\mathbf{k}$ & 1 & $\mathrm{~m}$ & $\mathrm{n}$ & 0 & $n$ & $q$ & $r$ & 8 & $t$ & $\mathbf{u}$ & $\mathbf{v}$ & $\mathbf{w}$ & $x$ & $\mathbf{y}$ & $\mathbf{z}$ & $-a$ & b & $c$ \\
\hline$c$ & $\mathrm{f}$ & $g^{\prime}$ & $\mathbf{h}$ & i & $\mathbf{k}$ & 1 & $\mathrm{~m}$ & $\mathrm{n}$ & 0 & $\mathbf{p}$ & $q$ & $\mathbf{r}$ & $s$ & $t$ & $\mathbf{n}$ & $\mathbf{v}$ & $w$ & $z$ & $\mathbf{y}$ & $\mathrm{z}$ & a & $\mathrm{b}$ & c & d \\
\hline$n$ & 0 & $\mathbf{p}$ & $q$ & $\mathbf{r}$ & $\mathbf{8}$ & $t$ & $\mathbf{u}$ & $\nabla$ & $\mathbf{w}$ & $\mathbf{x}$ & $\mathbf{y}$ & $\mathbf{z}$ & $a$ & $b$ & $c$ & $\mathrm{~d}$ & 6 & $\mathbf{f}$ & $g$ & $\mathbf{h}$ & i & $\mathbf{k}$ & 1 & $\mathbf{m}$ \\
\hline$t$ & $\mathrm{u}$ & $\checkmark$ & $w$ & $\mathbf{x}$ & $y$ & $\mathbf{z}$ & a & b & c. & d & e & $\mathrm{f}$ & $\mathrm{g}$ & $\mathbf{h}$ & i & $\mathbf{k}$ & 1 & $\mathbf{m}$ & $\mathbf{n}$ & 0 & $\mathbf{p}$ & $\mathbf{q}$ & $\mathbf{r}$ & 8 \\
\hline$i$ & $\mathrm{k}$ & 1 & $\mathbf{m}$ & $\mathbf{n}$ & 0 & $\mathbf{p}$ & $q$ & $r$ & $\mathrm{~s}$ & $t$ & $\mathrm{u}$ & $\nabla$ & $w$ & $x$ & $\mathrm{y}$ & $\mathrm{z}$ & $a$ & $\mathbf{b}$ & 0 & d & $\mathrm{e}$ & $\mathbf{f}$ & 8 & $\mathbf{b}$ \\
\hline$a$ & b & c & d & e & $\mathbf{f}$ & g & A & $\mathbf{i}$ & $\mathbf{k}$ & 1 & $\mathrm{~m}$ & $\mathbf{n}$ & 0 & 2 & $q$ & $\mathbf{r}$ & 8 & $t$ & $\mathbf{u}$ & $\nabla$ & $\mathbf{T}$ & $\mathbf{x}$ & $\mathbf{y}$ & 2 \\
\hline
\end{tabular}

The St Cyr scheme is essentially the same as Gronfeld's. For instance, in the St Cyr system the key word gibe leads to the same result as the key number 6814 by Gronfeld's method. One advantage of the St Cyr system over that advocated by Gronfeld is that key words are more easily recollected than key numbers. Another advantage comes from the fact that the employment of words is equivalent to using 26 digits instead of 10: thus the key word kings is equivalent to a number whose digits, from left to right, are 11, 9, 14, 7, 19. Messages in this cipher of any considerable length can be read by the same rules as are used to discover the key in Gronfeld's code. To hamper a decipherer I recommend the introduction, as in Gronfeld's method, of a non-significant letter after every $m$ th letter.

The Beaufort Cipher, introduced in the British Navy by Admiral Beaufort in 1857, is of the St Cyr type. Its inventor thought it insoluble, but French writers have shown that no special difficulties occur in the discovery of the key word in the solution, though the analysis is tedious.

A better system of this kind is the Playfair Cipher. In this 25 cells arranged in the form of a square are filled by the letters of a key word such as Manchester (striking out any 
letter which occurs more than once) and the remaining letters of the alphabet, thus :-

\begin{tabular}{|c|c|c|c|c|}
\hline$m$ & $a$ & $n$ & $c$ & $h$ \\
\hline$e$ & $\cdot$ & $t$ & $r$ & $b$ \\
\hline$d$ & $f$ & $g$ & $i$ & $j$ \\
\hline$k$ & $l$ & 0 & $p$ & $u$ \\
\hline$v$ & $w$ & $x$ & $y$ & $z$ \\
\hline
\end{tabular}

Only 25 cells are available, so $k$ has been used for both $k$ and $q$. The message is then divided into pairs of consecutive letters, but to prevent any pair consisting of the same two letters, a dummy letter like $z$ is, when necessary, introduced. If both letters of a pair appear in the same vertical (or horizontal) line of the square, each of them is replaced by the letter in the square immediately to its right (or below it)-the letters in every line being treated as in cyclical order. If the letters in a pair do not appear in the same line in the square they must necessarily be at opposite angles of some rectangle, and they are replaced by those at the other angles of the rectangle, each by that which is in the same horizontal line. Thus the message will meet you at noon would first be written wi $l z l m$ ez et yo $u a$ tn oz on; then be put in cipher as yf uw ka bv sr xp lh gt ux $p c$; and finally be sent as yfuwk abvsr xplhg tuxpc. It is curious that this cipher is not used more extensively, for the discovery of the key is difficult, even to specialists.

Fourth Type of Ciphers. A cipher of the fourth type is one in which each letter is always represented by the same symbol, but more than one letter may be represented by the same symbol. Such ciphers were not uncommon at the beginning of the nineteenth century, and were usually framed by means of a key sentence containing about as many letters as there are letters in the alphabet. 
Thus if the key phrase is The fox jumped over the garden gate, we write under it the letters of the alphabet in their usual sequence as shown below:

The fox jumped over the garden gate. $a b c d e f g h i j k l$ mnop qrs tuvwxy $z a b c$. Then we write the message replacing $a$ by $t$ or $a, b$ by $h$ or $t, c$ by $e, d$ by $f$, and so on. Here is such a message. $M$ foemho nea ge eoo jmdhohg avf teg ev ume afrmeo. But it will be observed that in the cipher $a$ may represent $a$ or $u, d$ may represent $l$ or $w, e$ may represent $c$ or $k$ or $o$ or $s$ or $x, g$ may represent $t$ or $z, h$ may represent $b$ or $r, o$ may represent $e$ or $m, r$ may represent $p$ or $v$, and $t$ may represent $a$ or $b$ or $q$. And the recipient, in deciphering it, must judge as best he can what is the right meaning to be assigned to these letters when they appear.

An instance of a cipher of the fourth type is afforded by a note sent by the Duchesse de Berri to her adherents in Paris, in which she employed the key phrase

$$
\begin{aligned}
& l e g o u v e r n e m e n t \text { provisoire. } \\
& a b \text { cdefghijklmn opqrstuvxy. }
\end{aligned}
$$

Hence in putting her message into cipher she replaced $a$ by $l$, $b$ by $e, c$ by $g$, and so on. She forgot however to supply the key to the recipients of the message, but her friend Berryer had little difficulty in reading it by the aid of the rules I have indicated, and thence deduced the key phrase she had employed.

Desiderata in Cryptographs and Ciphers. Having mentioned various classes of cryptographs and ciphers, I may add that the shorter a message in cryptograph, the more easily it is read. On the other hand, the longer a message in cipher, the easier it is to get the key. In choosing a cipher for practical purposes, which will usually imply that it can be telegraphed or telephoned, we should seek for one in which only current letters, symbols, or words are employed; such that its use does not unduly lengthen the message; such that the key to it can be reproduced at will and need not be kept in a form which 
might betray the secret to an unauthorized person; such that the key to it changes or can be changed at short intervals; and such that it is not ambiguous. Many ciphers of the second and third types fulfil these conditions; in particular the Gronfeld or St Cyr Method, or the Playfair Cipher, may be noted. A cipher written cryptographically, or a cryptograph written in cipher, or a cipher again enciphered by another process, is almost insoluble even by experts, unless accidents reveal something in the construction, but it is troublesome to make, and such elaborate processes are suited only for the study, where the time spent in making them up and deciphering them is not of much consequence.

Cipher Machines. The use of instruments giving a cipher, which is or can be varied constantly and automatically, has been often recommended*. The possession of the key of the instrument as well as a knowledge of the clue word is necessary to enable anyone to read a message, but the risk of some instrument, when set, falling into unauthorized hands must be taken into account. Since equally good ciphers can be constructed without the use of mechanical devices I do not think their employment can be recommended.

On the Solution of Cryptographs and Ciphers. Much ingenuity has been shown in devising means for reading messages written in cryptograph or cipher. It is a fascinating pursuit, but I can find space for only a few remarks about it.

In such problems we must begin by deciding whether the message is a cryptograph or a cipher. If it is a combination of both, the problem is one of extreme difficulty, and is likely to baffle anyone but a specialist, but such combinations are unusual, and most secret messages belong to one class or the other.

If the scale of frequency of the letters agrees generally with Conrad's Table, presumably the message is in cryptograph,

- See, for instance, the descriptions of those devised by Sir Charles Wheatstone, given in his Scientific Papers, London, 1879, pp. 342-347; and by Capt. Bazeries in Comptes Rendus, Association Français pour l'Avancement des Sciences, vol. $\mathrm{xx}$ (Marseilles), 1891, p. 160 et seq. 
though we must allow for the possibility that dummy letters, like $j, k, x$, and $z$, have been introduced either to separate words or deliberately to confuse those not in the secret. A short sentence of this kind may be read by an amateur, but only an expert is likely to discover the key to a long and well constructed cryptographic message.

If the message is long enough, say about 80 words, and the scale of frequency of particular letters differs markedly from Conrad's scale, there is a presumption that the message is in cipher. If the numbers of the two scales agree generally, probably a simple substitution alphabet has been used, i.e. it is a cipher of the first type, and generally the discovery of the key is easy. If it is not a cipher of this type, we must next try to find whether it is of any of the other recognised types. The majority of other ciphers are included in Gronfeld's number (or the St Cyr word) system, and here I will confine myself to a discussion of how such ciphers may be read.

The discovery of a key to a cipher of this kind is best illustrated by a particular case. I will apply the method to the message cisvg vumya vijnp vgzsi ybpjp woiy. This is an example of a Gronfeld's cipher with no additional complications introduced, but the message is short, and it so happens that the letters used are not in the normal scale of frequency; yet it can be read with ease and certainty.

The first thing is to try to find the number of digits in the key number. Now we notice that the pair of letters $v g$ occurs twice, with an interval of 12 . If in each case these represent the same pair of letters in the original message, the number of digits in the key number must be 12 or a divisor of 12 . Again the pair of letters $i y$ occurs twice, with an interval of 8 , and this suggests that the number of digits in the key number is 8 or a divisor of 8. Accordingly we conjecture that the key number is one of either 2 or 4 digits: this conclusion is strengthened by noting the intervals between the recurrences of the same letters throughout the message. We may put 2 on one side till after we have tried 4, for anyone using Gronfeld's method would be unlikely to employ a key number less 
than 100. Accordingly we first try 4, and if that fails try 2. Had no clue of this kind been obtained from the recurrence of a pair of letters, we should have had to try successively making the key numbers comprise $2,3,4,5, \ldots$ digits, but here (and in most messages) a cursory examination suggests the number of digits in the key number. We commence then by assuming provisionally that the key number has 4 digits. Accordingly we must now re-write our message in columns, each of 4 letters, giving altogether 4 lines, thus:

$$
\begin{aligned}
& \text { c } g y j g y p y \\
& i v a n z b w \\
& s u v p s p o \\
& v m i v i j i
\end{aligned}
$$

If the Gronfeld method was used, the letters in each of these lines were obtained from the corresponding letters in the original message by a simple substitution alphabet. Had the message been long we could probably obtain this alphabet at once by Conrad's Table. Here, however, the message is so short that the Table is not likely to help us decisively, and we must expect to be obliged to try several shifts of the alphabet in each line.

In the first line $y$ occurs three times, and $g$ twice. According to Conrad's Table, the most common letters in English are $e, t, a, 0, i, n, s, r, h$. Probably $y$ stands for one of these and $g$ for another. If $y$ is made to stand successively for each of these, it is equivalent to putting every letter $\theta$ places backward, where $\theta$ is successively $20,5,24,10,16,11,6,7,17$. Similarly, making $g$ stand successively for $e, t, a, 0, i, n, s, r, h$, we have $\theta$ equal to $2,13,6,18,24,19,14,15,25$. Altogether this gives us 16 systems for the representation of the first line. We might write these out on 16 slips, and provisionally reject any slip in which many unusual letters appear, but obviously, the most probable hypothesis is that where $y$ stands for $s$, and $g$ for $a$, both of which changes give $\theta=6$, or that where $y$ stands for $a$, and $g$ for $i$, both of which changes give $\theta=24$ : these give for the first line either $w, a, s, d, a, s, j, s$, or $e, i, a, l$, $i, a, r, a$. 
In the second line no letter occurs more than once, so we get no clue from Conrad's Table. This could not happen if the message were of any considerable length.

In the third line $p$ occurs twice, and $s$ twice. Hence, as before, we must make $p$ and $s$ successively stand for the letters $e, t, a, 0, i, n, s, r, h$. These give respectively $\theta=11,22,15,1$, $7,2,23,24,8$, and $\theta=14,25,18,4,10,5,0,1,11$. Altogether this gives us 16 systems for the representation of this line. Obviously the most probable hypothesis is that where $\theta=11$, $p=e, s=h$, or that where $\theta=1, p=o$, and $s=r$ : these give for the third line either $h, j, k, e, h, e, d$, or $r, t, u, o, r, o, n$.

In the fourth line $i$ occurs three times. As before, make $i$ stand successively for $e, t, a, o, i, n, s, r, h$. Of these the first, where $\theta=4$, is the most probable. The slip corresponding to this is $r, i, e, r, e, f, e$.

Now try combinations of these slips each in its proper line until, when we read the message in columns, we get the beginning of a word; if words appear in more than one column it is almost certain that we are right. We begin by taking the five slips which are indicated as being specially probable. The slip in the first line derived from $\theta=6$, the slip in the third line derived from $\theta=1$, and the slip derived from $\theta=4$ in the fourth line give w.rra.tis . ued . ora.res . ofj . nes, and of course the solution is obvious. The key number was 6814 , and the message is deciphered by using 6814 backwards. The corresponding St Cyr key word is gibe. The message was Warrant issued for arrest of Jones.

If the combination of the slips is troublesome we can sometimes get assistance by choosing those combinations which make the recurring pairs of letters (here $v g$ and $i j$ ) represent pairs which occur in Conrad's Table. Also the occurrence of double letters in the cipher will often settle what combinations of slips are possible.

It may be said that this is a tedious operation. Of course it is. Deciphering is bound to be troublesome, but a great deal of the work can be done by unskilled clerks working under the direction of experts. The longer the message, the fewer the 
slips we have to try, and had the above message been three times as long, we could have solved the problem with half the trouble. The above example was not complicated by employing dummy letters or artificial alphabets: their use increases the difficulty of the decipherer, but if the message is a long one, the difficulties are not insuperable. Specialists, especially if working in combination, are said to select the right methods with almost uncanny quickness.

This chapter has already run to such a length that I cannot find space to describe more than one or two ciphers that appear in history.

It is said that Julius Caesar in making secret memoranda was accustomed to move every letter four places forward, writing $d$ for $a, e$ for $b, \& c$. This would be a very easy instance of a cipher of the first type, but it may have been effective at that time. His nephew Augustus sometimes used a similar cipher, in which each letter was moved forward one place*.

Bacon proposed a cipher in which each letter was denoted by a group of five letters consisting of $A$ and $B$ only. Since there are 32 such groups, he had 6 symbols to spare, which he could use to separate words or to which he could assign special meanings. A message in this cipher would be five times as long as the original message. This may be compared with the far superior system of the five (or four) digit codebook system in use at the present time.

In the Morse code employed in telegraphy, as in the Baconian system, only two signs are used, commonly a dot or a short mark or a motion to the left, and a dash or a long mark or a motion to the right. The Morse Alphabet is as follows: $a(\cdot-), b(-\cdots), c(-\cdot-\cdot), d(-\cdot), e(\cdot)$, $f(\cdot-\cdot), g(--\cdot), h(\cdot \cdots), i(\cdot), j(\cdot---), k(-\cdot-)$, $l(\cdot-\cdot \cdot), m(-\longrightarrow), n(-\cdot), o(--\longrightarrow), p(\cdot-\cdots), q(-\cdots \cdot-)$, $r(\cdot-\cdot), s(\cdots), t(-), u(\cdot-), v(\cdots-), w(\cdot--), x(-\cdots-)$,

* Of some of Caesar's correspondence, Suetonius says (cap. 56) si quis investigare et persequi velit, quartam elementorum literam, id est, d pro a, et perinde reliquas commutet. And of Augustus he says (cap. 88) quoties autem per notas scribit, $b$ pro $a, c$ pro $b$, ac deinceps eadem ratione, sequentes literas ponit; pro $x$ autem duplexi $a$. 
$y(-\cdot--), z(--\cdot)$. Since there are 30 possible permutations of two signs taken not more than four together, this leaves four signals unemployed, (- - - ), (- - - ), $(\cdot--),(\cdot-\cdot-)$, which might have been utilized for special signals. In telegraphy there are also recognized signs or combinations for numerals, for the ends of words and messages, and for various calls between the sender and the recipient of a message.

Charles I used ciphers freely in important correspondence -the majority being of the second type. He was foolish enough to take a cabinet, containing many confidential notes in cipher, with him to the field of Naseby, where they fell into the hands of Fairfax*. In these papers each letter was represented by a number. Clues were provided by the King who had written over the number the letter which it represented. Thus in two letters written in 1643, $a$ is represented by 17 or $18, b$ by $13, c$ by 11 or $12, d$ by $5, e$ by 7 or 8 or 9 or 10 , $f$ by 15 or $16, g$ by $21, h$ by 31 or $32, i$ by 27 or $28, k$ by 25 , $l$ by 23 or $24, m$ by 42 or $44, n$ by 39 or 40 or $41, o$ by 35 or 36 or 37 or $38, p$ by 33 or $34, r$ by 50 or 51 or $52, s$ by 47 or 48 , $t$ by 45 or $46, u$ by 62 or $63, w$ by 58 , and $y$ by 74 or 77 . Numbers of three digits were used to represent particular people or places. Thus 148 stood for France, 189 for the King, 260 for the Queen, 354 for Prince Rupert, and so on. Further, there were a few special symbols, thus $k 1$ stood for of, $n 1$ for to, and $f 1$ for $i s$. The numbers 2 to 4 and 65 to 72 were nonsignificant, and were to be struck out or neglected by the recipient of the message. Each symbol is separated from that which follows it by a full-stop.

A similar, though less elaborate, system was used by the French in the Peninsular War. An excellent illustration of the inherent defects of this method is to be found in the writings of the late Sir Charles Wheatstone. A paper in cipher, every page of which was initialed by Charles I, and countersigned by Lord Digby, was purchased some years

* First Report of the Royal Commission on Historical Manuscripts, 1870, pp. $2,4$. 
ago by the British Museum. It was believed to be a state paper of importance. It consists of a series of numbers without any clue to their meaning, or any indication of a division between words. The task of reading it was rendered the more difficult by the supposition, which proved incorrect, that the document was in English; but notwithstanding this, Sir Charles Wheatstone discovered the key*. In this cipher $a$ was represented by any of the numbers $12,13,14,15,16$, or $17, b$ by 18 , 19 , and so on, while some 65 special words were represented by particular numbers: in all about 150 different symbols were used.

The famous diary of Samuel Pepys is commonly said to have been written in cipher, but in reality it is written in shorthand according to a system invented by J. Sheltont. It is however somewhat difficult to read, for the vowels are usually omitted, and Pepys used some arbitrary signs for terminations, particles, and certain words-so far turning it into a cipher. Further, in certain places, where the matter is such that it can hardly be expressed with decency, he changed from English to a foreign language, or inserted non-significant letters. Shelton's system had been forgotten when attention was first attracted to the diary. Accordingly we may say that, to those who first tried to read it, it was written in cipher, but Pepy's contemporaries would have properly described it as being written in shorthand, though with a few modifications of his own invention.

A system of shorthand specially invented for the purpose is a true cipher. Such a system in which the letters were represented by four strokes varying in length and position was employed by Charles I. Another such system in which each letter is represented either by a dot or by a line of constant length was used by the Earl of Glamorgan, better known by his subsequent title of Marquess of Worcester, in 1645; each of these

* The document, its translation, and the key used, are given in Wheatstone's Scientific Papers, London, 1879, pp. 321-341.

+ Tachy-graphy, by J. Shelton, frst edision 1690, sixth edition used by Pepys 1641. A somewhat similar system by W. Cartwright was issued by J. Rich under the title Semographie, London, 1644. 
was a cipher of the first type and had the defects inherent in almost every cipher of this kind: in fact Glamorgan's letter was deciphered, and the system was discovered by H. Dircks*. Obsolete systems of shorthand $\nmid$ may be thus used as ciphers.

It is always difficult to read a very short message in cipher, since necessarily the clues are few in number. When the Chevalier de Rohan was sent to the Bastille, on suspicion of treason, there was no evidence against him except what might be extracted from Monsieur Latruaumont. The latter died without making any admission. De Rohan's friends had arranged with him to communicate the result of Latruaumont's examination, and accordingly in sending him some fresh body linen they wrote on one of the shirts $M g$ dulhxcclgu ghj yxuj, lm ct ulgc alj. For twenty-four hours de Rohan pored over the message, but, failing to read it, he admitted his guilt, and was executed November 27, 1674. The cipher is a simple one of the first type, but the communication is so short that unless the key were known it would not be easy to read it. Had de Rohan suspected that the second word was prisonnier, it would have given him 7 out of the 12 letters used, and as the first and third words suggest the symbols used for $l$ and $t$, he could hardly have failed to read the message.

Marie Antoinette used what was in effect a St Cyr cipher, consisting of 11 substitution alphabets employed in succession. The first alphabet was $n, o, p, \ldots . . z, a, b, \ldots . . l, m$; the next, $o, p, q, \ldots \ldots m, n$; the next $p, q, r, \ldots \ldots n, o$; and so on. An expert would easily read a message in this cipher.

One of the systems in use to-day is the five digit codebook cipher, to which I have already alluded. In this, a code dictionary is prepared in which every word likely to be used is printed, and the words are numbered consecutively 00000, $00001, \ldots$ up, if necessary, to 99999. Thus each word is

- Life of the Marquis of Worcester by H. Dircks, London, 1865. Worcester's system of shorthand was described by him in his Century of Inventions, London, 1663 , sections $3,4,5$.

+ Various systems, inoluding those used in olsssical and medieval times, are described in the History of Shorthand by T. Anderson, London, 1882. 
represented by a number of five digits, and there are $10^{\circ}$ such numbers available. The message is first written down in words. Below that it is written in numbers, each word being replaced by the number corresponding to it. To each of these numbers is added some definite pre-arranged clue number-the words in the dictionary being assumed to be arranged cyclically, so that if the resulting number exceeds $10^{5}$ it is denoted only by the excess above $10^{5}$. The resulting numbers are sent as a message. On receipt of the message it is divided into consecutive groups of five numbers, each group representing a word. From each number is subtracted the pre-arranged clue number, and then the message can be read off by the code dictionary. If and when such a message is published, the construction of the sentences is usually altered before publication, so that the key may not be discoverable by anyone in possession of the codebook or who has seen the cipher message. This is a rule applicable to all cryptographs and ciphers.

This is a cipher with $10^{5}$ symbols, and as each symbol consists of five digits, a message of $n$ words is denoted by $5 n$ digits, and probably is not longer than the message when written in the ordinary way. Since however the number of words required is less than $10^{5}$, the spare numbers may be used to represent collocations of words which constantly occur, and if so the cipher message may be slightly shortened.

If the clue number is the same all through the message it would be possible by not more than $10^{5}$ trials to discover the message. This is not a serious risk, but, slight though it is, it can be avoided if the clue number is varied; the clue number might, for instance, be 781 for the next three words, 791 for the next five words, 801 for the next seven words, and so on. Further it may be arranged that the clue numbers shall be changed every day; thus on the seventh day of the month they might be 781, 791, \&c., and on the eighth day 881,891 , \&c., and so on.

This cipher can however be further improved by inserting at some step, say after each $m$ th digit, an unmeaning digit. For example, if, in the original message written in numbers, we 
insert a 9 after every seven digits we shall get a collection of words (each represented by five digits), most of which would have no connection with the original message, and probably the number of digits used in the message itself would no longer be a multiple of 5 . Of course the receiver has only to reverse the process in order to read the message.

It is however unnecessary to use five symbols for each word. For if we make a similar code with the twenty-six letters of the alphabet instead of the ten digits, four letters for each word or phrase would give us $26^{4}$, that is, 456976 possible variations. Thus the message would be shorter and the power of the code increased. Further, if we like to use the ten digits and the twenty-six letters of the alphabet-all of which are easily telegraphed-we could, by only using three symbols, obtain $36^{3}$, that is, 46656 possible words, which would be sufficient for all practical purposes.

This code, at any rate with these modifications, is undecipherable by strangers, but it has the disadvantages that those who use it must always have the code dictionary available, and that it takes a considerable time to code or decode a communication. For practical purposes its use would be confined to communications which could be deciphered at leisure in an office.

NOTE. Mr C. H. Harrison has pointed out that an objection to the Playfair system described above on pages 308,309 is that if a particular word is repeated, its central letters can appear in the cipher in only two forms. If this word is a long one and it is guessed correctly the key-word can generally be found.

Mr Harrison has devised a slide-rule eipher of the complex shifting substitution type but such that the recurrence of the shifts is non-cyclioal. I have not space to describe it here, but it seems safer than most of those mentioned in this chapter, and its use, in an office with intelligent clerks, would present no difficulty. I am not however convinced that it would be easier or safer to use than a simple cryptographio cipher. (See p. 293.) 


\section{CHAPTER XV.}

\section{STRING FIGURES.}

In the five editions of this work issued between 1911 and 1920 I devoted a chapter of some length to describing the production of string figures. In $1920 \mathrm{I}$ gave an account of the subject in a lecture in London, and this, with the addition of a good many examples, has been issued as a small booklet. That being now available*, I propose in this edition to cut down the space devoted to the subject and merely explain the construction of a few typical string forms which may serve as an introduction to the amusement and will, I would fain hope, induce my readers to go further into it.

The formation of these designs is a world-wide amusement of primitive man and has an interesting history. Such figures, when shown to a few spectators in a room, always prove, as far as my experience goes, interesting alike to young and old; but their attractiveness, their fascination I might almost say, is not permanent unless people can be induced to construct them for themselves, and, bold though I may seem, I venture to assert that those who learn the moves will assuredly find pleasure in the game. In most cases a design takes less than five minutes to learn if a practical lesson be given, and not more than a quarter of an hour if it has to be puzzled out and memorized from a complete description. Much of the charm of the art lies in the ease and rapidity with which the figures are produced; to make any of these here described rarely occupies more than eight or ten seconds when once the construction has been mastered.

A string figure is usually made by taking a piece of good

* String Figures, An Amusement for Everybody, by W. W. Rouse Ball, Cambridge, Second Edition, 1921. 
flexible string, such as macrami thread, about six-and-a-half feet long, knotting the ends so as to make it into a closed loop, and then weaving or twisting this loop on the fingers in some defined way. Normally at the end of each movement the hands should be drawn apart so as to tighten the string, and be held upright with the fingers pointing upwards and the palms approximately facing one another.

Here are some examples chosen purposely from widely separated countries. The figures are drawn as seen by the operator.

A Mosquito or Fly. This design, made in slightly different ways in different countries, represents the insect with its body midway between the hands, and its wings spread out. The following construction is taken from South American Indians whose habitat is on the banks of the Amazon.

This figure is there made by the following five successive movements: (1) Put the thumbs, held upright, into the loop of string, and separate the hands. (2) Move the left hand to a horizontal position; then turn it counter-clockwise under the strings and up towards you into its normal position, thus giving two dorsal strings and no palmar string. (3) Pass the right hand between you and the left hand, then put the right little-

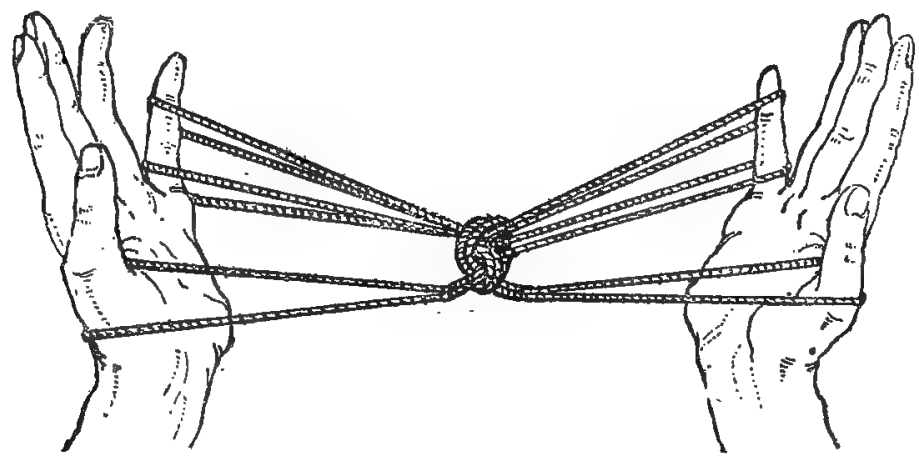

Figure i. The Fly.

finger from above under the dorsal strings, pick them up, and return the finger to its former position. [Unless the contrary 
is stated, such a return is always made when a string has been picked up.] (4) Pass the left hand between you and the right hand, then put the left little-finger on the right palm, and pass it towards you under the two strings on the right thumb, pick them up, and return. (5) Lastly, lift the dorsal strings on the left hand over the five digits, and separate the hands. This is the Mosquito.

With a mosquito between your hands you naturally try to squash it by clapping them together sharply. Do this, then draw the hands apart quickly, at the same time secretly release the little-fingers, and you will find that the insect has escaped, a result that usually happens in real life.

Like figures are made in various countries, and the tale is told differently in different places. Thus in Lifu in a similar figure, the twist in the string midway between the hands is taken to represent a coco-nut, and the last movement is used to illustrate efforts to crush the shell. The unskilful person, when clapping his hands and not releasing his little-fingers, fails, for on separating his hands the nut remains visible. But when the skilled native tries, then on clapping his hands and simultaneously releasing his little-fingers he succeeds, for on separating his hands the nut is broken and gone.

Such a use of string figures to illustrate local stories is not infrequent, and I think adds to their interest, but the amusement of malsing them is independent of their employment for this purpose.

A Man Climbing a Tree. This, derived from the Blacks in Queensland, is an example illustrative of action. The result shows two upright strings which represent the trunk of a tree, and loops round it which represent the arms and feet of a man climbing up it.

This figure may be made thus: (1) Put the tips of the left thumb and little-finger together, then put them from below into the loop of string and separate them: do the same with the right hand. Pick up on the back of the right index-finger the string resting on the left palm, and return; similarly pick up the right palmar string on the back of the left index-finger. 
[These movements are a common beginning, and are known as Opening A. Opening B is obtained in the same way, save that, in

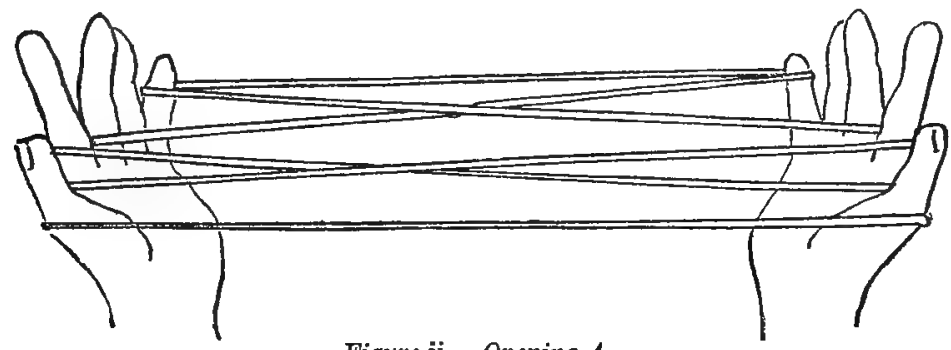

Figure ii. Opening A.

the second part of the Opening, the right palmar string is taken up by the left index before the left palmar string is taken up by the right index. In most of the figures described below it is immaterial whether we begin with the Opening A or Opening B.] (2) Bend each little-finger down towards you over four strings, with its back pick up the next string, and return. (3) Lift up

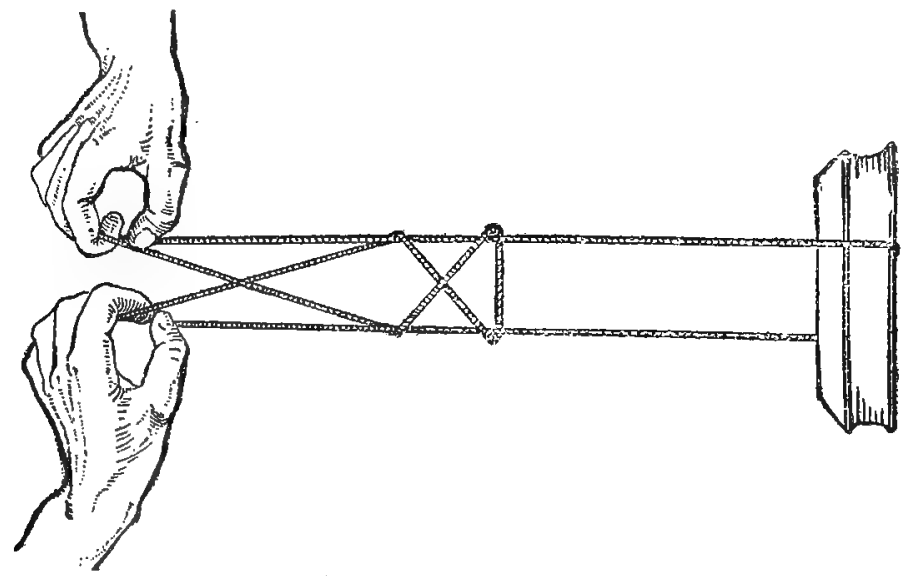

Figure iii. A Man Climbing a Tree.

the lower of the two loops on the left little-finger, pull it over the upper loop, then over the tip of the finger, and then drop it on the palm of the hand: do the same with the right hand. [This is known as Navahoing the loops.] (4) Bend each indexfinger over the palmar string and between the two strings of 
the loop on that finger and press its tip on the palm. (5) Holding the strings loosely, slip the loops off the thumbs; then still keeping the tips of the index-fingers pressed on the palms, separate the hands, thus causing the loops near the bases of those fingers to slip over the knuckles and so off the fingers. (6) Lastly, put the far little-finger string under one foot, or under a heavy book, release the little-fingers, and pull gently with the index-fingers, after hooking their tips into the string they hold. This makes the man climb the tree.

A Tent Flap. This figure is due to the Apache Red Indians who once occupied a large tract of land in the Eastern States of America. The tribe is now almost extinct, but the figure is familiar to various natives who say that originally they got it from Apaches living on the Reservation Lands maintained by the United States Government. The result shows a pretty piece of net-work which looks like a hurdle, but is said to have been intended to represent the flap or perhaps the lacing covering the opening to a tent.

This figure may be made thus: (1) Opening A. (2) Lift the loops off the index-fingers, pass them over their corresponding hands on to the wrists, thus making them dorsal strings. (3) Bend each thumb away from you over one string, and with its back pick up from below the next string, and return. Bend each little-finger towards you, and with its back pick up the next string. (5) Grasp with the left hand all the strings in

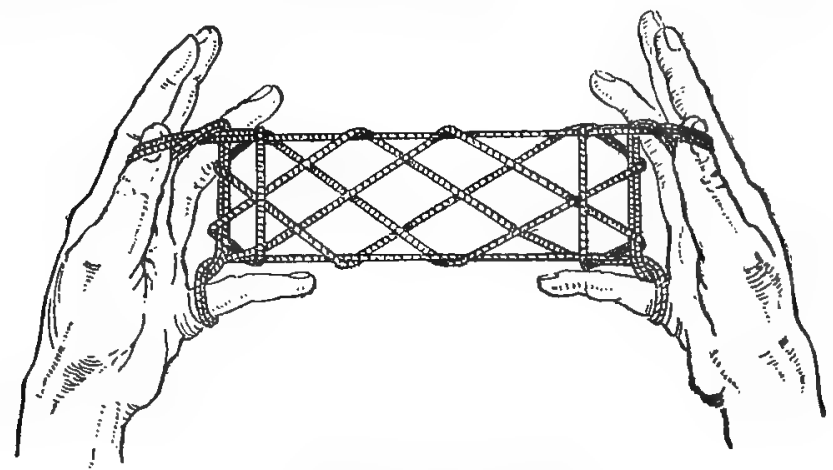

Figure iv. A Tent Flap. 
the centre of the figure where they cross, pass this bunch of strings between the right thumb and index-finger so that the bunch lies along the arm, with the left thumb and index-finger take hold of the two loops on the right thumb, draw them over the tip of the right thumb to the palmar side, and then replace the two loops on the right thumb; make a similar movement with the other hand. (6) Lastly, lift the wrist loops over the hands, letting them fall on the front or palmar sides of the hands, rub the hands together, separate them sharply, and the figure will appear.

Lightning. This figure was obtained from the Navaho Indians who live in the far West, on the Mexican border of Arizona, where the customs of the Red Man have not yet been destroyed by civilization and law. The final presentation of the zig-zag lightning should come quite suddenly, and to a stranger unexpectedly.

The figure may be made thus: (1) Put the string in the form of a figure of eight, one oval (preferably small) lying away from you, and the other towards you, and the strings crossing in the middle of the figure; then put the index-fingers down into the far oval, and the thumbs down into the near oval; next separate the hands and then turn them up into their normal position with the thumbs and fingers well spread out, thus causing the strings of the loops on the thumbs and index-fingers to cross one another. (2) Bend each thumb away from you over two strings and with its back pick up from below the next string, i.e. the far index string, and return. (3) Bend each mid-finger towards you over one.string, and with its back pick up from below the next string. (4) Bend each ring-finger towards you over one string, and with its back pick up from below the next string. (5) Bend each little-finger towards you over one string, and with its back pick up from_below the next string. (6) Move the thumbs away from you, and place their tips in the spaces by the little-fingers, their fronts resting on the near little-finger string; this releases the thumb loops: the figure in this form may be taken to represent thunder clouds. (7) Lastly, throw the loops thus released over the other strings, and at the same 
time with the thumbs press down the near little-finger string sharply and as far as possible, and the figure representing zig-

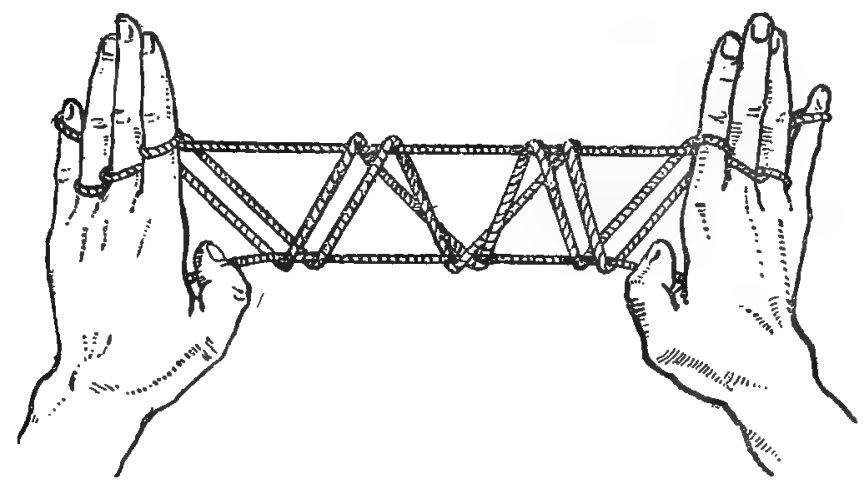

Figure v. Lightning.

zag lightning flashes out: the final movement should be made as rapidly as possible, it is less easy than most of the moves here described. As is the case in many of these figures the resulting pattern is shown stretched or hung on parallel horizontal strings, and to present it effectively, it is desirable to keep these strings widely separated.

A Salmon Net. This figure was obtained from the once German Caroline Isles, in the Pacific. The result is a design of three diamond-shaped lozenges strung in line between two horizontal strings: this is taken to be a net in which an object representing a salmon gets entangled.

This figure may be made thus: (1) Opening A. (2) Take the right hand out of the string, and put the tips of the right thumb and little-finger together from the right side into the left index loop, extend, and release the left index. (3) With the back of the right index-finger pick up the string on the palm of the left hand. (4) Bend each thumb from you over one string, and with its back pick up the next string, keeping it on the tip of the thumb. (5) Bend each index-finger towards you, and with the extreme tip of its back pick up the next string. Navaho the thumb loops. (7) Lastly, release the little-fingers, rotating the hands so as to face away from you, and extend. 
An onlooker is now invited to put his hand, representing a salmon, in the middle mesh. It escapes if the left hand is re-

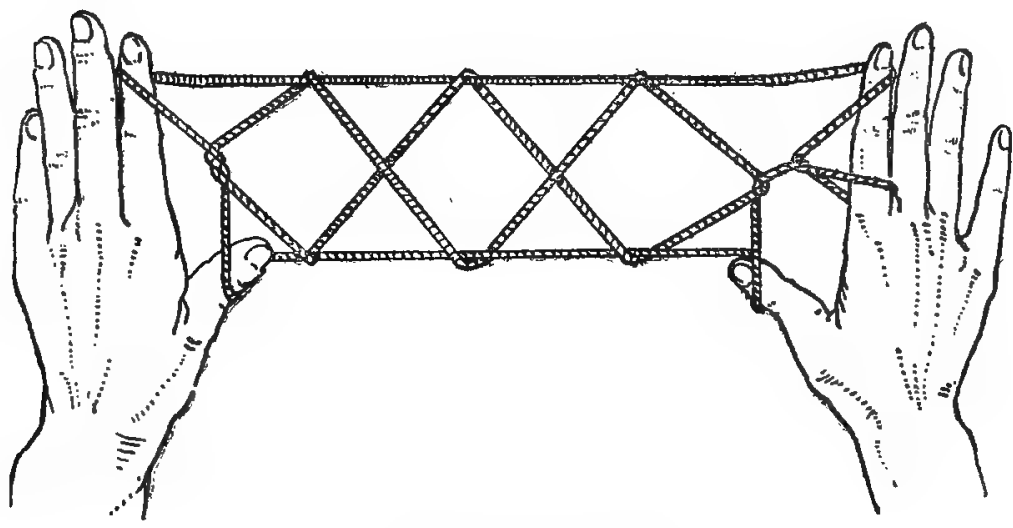

Figure vi. A Salmon Net.

leased and the right hand moved away, but is caught if the right hand is released and the left hand moved away.

The Yam Thief. This figure has been found in Northern Europe, Africa, America, Oceania and Northern Asia. Everywhere it is used to illustrate a story.

The figure may be made thus: (1) Hold the left hand open with the palm facing you, and the thumb upright and the fingers pointing to the right and slightly upwards. With the right hand, loop the string over the left thumb, crossing the strings if you like, and let one string hang down over the palm and the other over the back of the hand-we may call these the palmar and dorsal strings. (2) Pass the right index-finger from below under the palmar string, and then between the left thumb and index-finger, and with its front tip hook up a loop of the dorsal string; pull this loop between the left thumb and index-finger back on to the left palm; then with the right index-finger give the loop one twist clockwise, and put it over the palmar string on to the left index-finger; pull the two pendant strings so as to tighten the loops on the thumb and index-finger. (3) In the same way pass the right index-finger from below under the 
pendant palmar string, and then between the left index and middle fingers, and with its front tip hook up another piece of

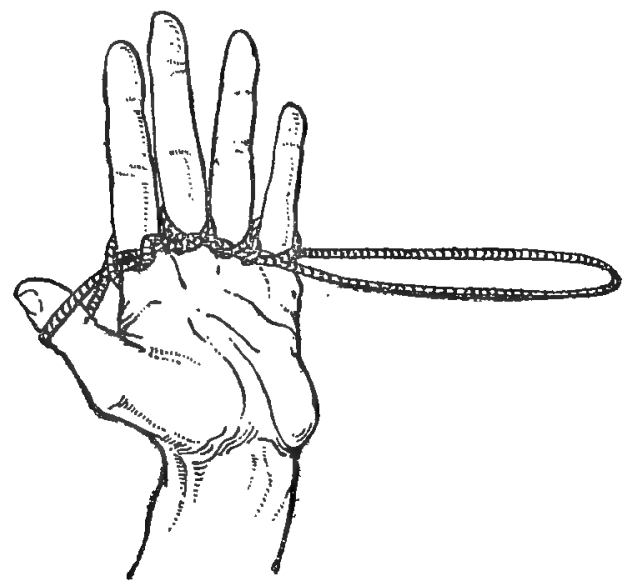

Figure vii. The Yam Thief.

the pendant dorsal string; pull this loop back on to the left palm, and with the right index-finger give the loop one twist clockwise, and put it over the palmar string on the left midfinger. (4) In the same way, working between the middle and ring fingers, hook up another loop of the pendant dorsal string, and put it on the left ring-finger. (5) In the same way, working between the ring and little fingers, pick up another loop of the pendant dorsal string, and put it on the left little-finger. (6) Take off the left thumb loop, and hold it between the left thumb and index-finger : and, for the sake of effect, to show that the loops are still on the fingers, pull the pendant dorsal string. (7) Lastly, pull the pendant palmar string, and the figure will come off the hand.

You can make up the story much as you like. In one version, told by the negroes on the Gold Coast, the thumb loop represents the owner of a yam patch. He is supposed to be asleep. The loops successively taken up from the dorsal string and put on the fingers represent the yams dug up by a thief, and tied up in bags ready for carrying off. The loop coming off the thumb represents the owner waking and going to see what is the matter. 
He looks down the back of the hand, sees the yams collected for removal, notices that the dorsal string holds them tight, and looks about for the thief. The thief, who may be represented by a loop on the pendant palmar string, coming back for his booty, sees the owner, whereupon (pulling that string) be disappears with all the yams.

Other places, other tales. Among some tribes the result is said to represent the flight of a row of birds sitting on a rail and suddenly disturbed; among others it is used to illustrate a story about a cat and a mouse. In Lifu, the strings on the hand are said to represent the root of an alou tree and the palmar string a branch of it. Someone, representing a strong stupid man, takes hold of the branch, and though he pulls for all he is worth and is encouraged by the shouts of the onlookers, he cannot move the root: then someone else, representing the traditional cunning village clown, takes hold of the branch and (the thumb loop being released) the root comes up easily, to the ostensible astonishment of the spectators.

The Caterpillar. This design has been found in North Australia, and various places in Southern Oceania. The result shows the outline of a caterpillar which can be made to loop itself up and so crawl forward.

This figure may be made thus: (1) Put the tips of the left thumb and little finger together, then put them from below into the loop of string, and separate them; do the same with the right hand. (2) Put a loop round the left thumb. (3) With the back of the right index pick up the left thumb loop; pass the left index through the right index loop and, with its back, pick up the right palmar string; pass the right index through the left index loop, and with its back pick up the left palmar string. (4) Release the left hand; hold the right hand horizontal and palm downwards, and put the loop which is nearer the tip of the right index over the loop which is nearer the base of the finger. (5) Put the left little-finger and thumb towards you between the two loops on the right index and resting on the joint of the finger; and, with the back of the left little-finger, pick up the adjacent loop now nearer the base of the right index, 
and with the back of the left thumb, pick up the adjacent loop now nearer the tip of the right index; extend, thus pulling both

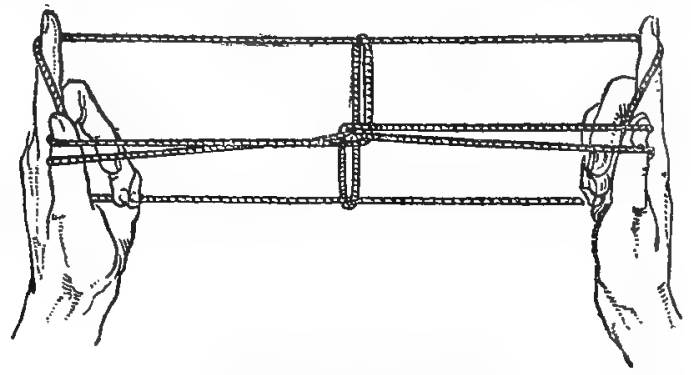

Figure viii. The Caterpillar.

loops off the right index. (6) Transfer each thumb loop to the corresponding index-finger, and then transfer it back again by putting the thumb from outside into the loop: the effect of this is to turn the thumb loops over. (7) Bend each thumb from you over one string, and, with its back, pick up the next string. (8) Put each index-finger over the palmar string under the far thumb string, and, with its back, pick up on the far tip of the finger the latter string, hold it against the index-finger by the mid-finger, erect the index-fingers, thus bringing the string connecting their tips to the top of the figure and rotate the wrists away from you. (9) Lastly, leeping the thumbs and inderfingers close together, bend down the little and ring fingers, and, with their tips, catch and stretch the far string, thus bringing it to the bottom of the figure. Extend flat on the knee, and the caterpillar appears.

If the wrists are now turned so as to move the palms of the hands upwards, the caterpillar will contract. Then turn them back to their former position, and he elongates. Repeat the action, and he walks down the leg.

Cat's Cradles. So far I have dealt with figures made by one man. I ought to mention that there is also a class of string figures, familiar in our nurseries, in which two players are concerned, each of them in turn taking the figure off the hands of the other. This form originated in Korea, and is not uncommon in the north of Europe. As usually presented the working is 
rather a dull affair, involving no skill, leading in succession to four designs known here as the cradle, a snuffer-tray, cat's-eye, and fish-in-a-dish, and in Korea as a hearse, a chess-board, a cow's-eye, and a rice-pestle. There are three or four standard moves, and by using them in various orders other figures can be deduced. I content myself with a bare allusion to this form.

String Tricks. Far more interesting than the Cat's Cradle of our nurseries are String Tricks which are a perennial source of pleasure to primitive men, lads, and those wise enough to retain interest in such things.

Here are a dozen examples, with references, for any reader who may like to try them: (1) The Lizard Twist, see below or C. F. Jayne, String Figures, New York, 1906, p. 337. (2) The Chippewa Release, Jayne, p. 346. (3) Cheating the Halter, see below or Jayne, p. 339. (4) The Threading a Closed Loop, see below or Jayne, p. 354. (5) The Fly on the Nose, see below or Jayne, p. 348. (6) The Joining the Ends of a Cut String, see almost any book on easy conjuring or parlour tricks, for instance, Hoffmann's Modern Magic, p. 317. (7) The Interlaced Handcuffs, see below or Hoffmann's Puzzles, p. 349. (8) The ButtonHole Trick (otherwise worked with a key or ring threaded on a closed loop), see below or Tom Tit's Scientific Amusements, London, 1919, p. 404. (9) The Knife in the Tree, see Tom Tit, p. 399. (10) The Reversed Twist, see Tom Tit, p. 400. (11) The Waistcoat Trick, see below. And (12) The Elusive Loop, see my String Figures, p. 50. Some of these are also described in K. Haddon's Cat's Cradles, London, 1911.

I add brief notes on the moves involved in some of these tricks. The presentation of the tricks may be left to the ingenuity of the operator: it is hardly necessary to add that for effectiveness the working should be quick and not be immediately repeated.

The Lizard Twist is worked thus: (1) Hold the string by the left hand, held rather high, the string hanging down on the right and left sides in a loop. (2) Put the right hand away from you through the loop. Bend the right hand down, and then turn it round the right pendant string clockwise; this will be done 
by pointing the fingers to the right, then towards you, and then upwards. (3) Keeping the fingers pointing upwards, move the right hand to the left between your body and the pendant strings, then clockwise beyond the left pendant string, then away from you, then to the right, and finally towards you through the loop. (4) Lastly, draw the hand down and to the right, and it will come free from the noose round the wrist.

The Chippewa Release is effected thus: (1) Put the loop on a vertical peg or on the upright index-finger of another person. (2) With the left-hand, take hold of the two strings of the loop (which are uncrossed, horizontal, and parallel) some foot or so away from the peg. (3) Put your right mid-finger from above to the left of the left-hand string, with the side of the finger catch that string, lift it up and carry it over the other string, and then put the finger down. (4) Move your right index-finger forward, and put it down near the peg, between the strings coming from the peg and as yet uncrossed. (5) Raise your mid-finger with the original left-hand string hanging on its tip, and place its tip on the peg. (6) Lastly, remove the index-finger, and at the same time pull with the left hand, whereon the string comes free.

The Cheating the Halter is worked thus: (1) Put your head through a loop of string, and let the rest of the loop hang down in front of you. (2) Pass the right string round the neck from the left side, draw the loop tight, and let it hang down in front of you. (3) Put the hanging loop on the hands, and form Opening A. (4) Pass the index loops over the head, and remove the hands and fingers from the other loops; a loop now hangs down in front of you. (5) Lastly, pull this loop, or either string of it, and all the strings will come off the neck.

We can vary the presentation by twisting the left string round the neck from the right side. In this case we must use Opening $\mathbf{B}$.

The Threading a Closed Loop, which is said to represent the eye of a needle, is effected thus: (1) Take hold of a piece of string, $A B C$, some three or four feet long, at a point $B$ about 8 inches from one end $A$. Hold this piece $A B$ in the left palm and hold the left hand so that the thumb points to the right. 
(2) Holding the other end, $C$, of the string with the right hand, wind the rest of the string $B C$, beginning with the $B$ end, round the left thumb, above the thumb when moving towards the body and under the thumb when moving away from the body. Leave about 6 inches at the end $C$ unwound. (3) Out of this 6 inches, make a small loop by carrying the end of $C$ held by the right hand to the far side of the rest of the loop. With the tips of the left thumb and index-finger hold this loop where the strings cross so that it stands erect. (4) Pick up with the right thumb and index-finger the end $A$ of the piece of string $A B$ which is in the left palm and open the left palm. Make this piece of string $A B$ taut, and carry the end of it $A$ to the right close under and between the left thumb and index. If this is properly done the piece $A B$ will pass near $B$ between the far and near portions of the cross of the loops, and then will be caught by the left thumb and index: thus between the thumb and index there are now three pieces of string. (5) Make passes with the end $A$ as if you were trying to thread it through the erect loop held by the left hand. (6) Lastly, pass the right hand sharply to the left over and beyond the left hand. This will carry the piece $A B$ beyond the two strings of the loop. Hence the loop which is still held up by the left thumb and index appears to have been threaded by the right hand string, but in reality the part of the string which hangs from the right hand is drawn between the left thumb and index up into the loop.

The Fly on the Nose is worked thus: (1) Hold the string at some point with the thumb and index-finger of one hand; and take hold of the string at a place, some 9 or 10 inches off, with the thumb and index of the other hand. (2) Make a small ring hanging down by passing the right hand to the left and on the near side of the string held by the left hand. There is now a long loop and a small ring, both hanging down, the right string of the ring being the left string of the loop. (3) Hold the place where the strings cross between the teeth, the string originally held by the right hand being on the lower side. Hold the strings or one string of the long loop with the left hand. (4) Place the right index from the far side through the ring, taking the ring 
string up to the root of the fingers. Close the right fist, and carry the fist (holding the ring) round in a circle, first back to the far side, then to the right, and so on round the right string of the loop, to its original position. (5) Open the index, keeping the rest of the fist shut, and put the tip of the index on the tip of the nose. (6) Finally, let go with the mouth and pull the left string of the loop. The whole string will then come away.

Of course the same result can be obtained if the ring is made by passing the right hand to the left on the far side of the string held by the left hand, so that when the cross is held in the mouth the string originally held by the right hand is uppermost. But in this case the ring should be taken by the left index, which is carried round the left string of the loop.

The Handcuff Trick is worked thus: Two players, $P$ and $Q$, are connected thus. One end of a piece of string is tied round $P$ 's right wrist, the other is tied round his left wrist. Another piece of string is passed through the space bounded by the string tied to $P^{\prime}$ 's wrist, his body, and his arms. The ends of this piece are then tied one on Q's right wrist, and the other on Q's left wrist. The players desire to free themselves from the entanglement.

Either player can free himself thus: (1) $P$ takes up a small loop $L$ near the middle of the string tied to his wrists, and pulling the loop to one of $Q$ 's wrists on the palmar side of it, passes it, from the elbow to the finger side, between $Q$ 's wrist and the loop on that wrist. (2) Next, $P$ draws this loop $L$ sufficiently far through until he is able to pass it, first over Q's hand, and then under the wrist loop on the outer side of $Q$ 's wrist, passing this time from the finger to the elbow side of $Q$. When in this position $P$ can pull his own string clear of $Q$ on the outer side of $Q$ 's arm.

The Button-Hole Trick is worked thus: (1) Pass a loop of string through a button-hole or key-ring. (2) Hold the thumbs upright, and insert them from below in the two loops one on each side of the button-hole. Move the hands a little out from the body and towards each other. (3) Hook the right littlefinger into the right-hand string of the left thumb loop, and pull it across to the right hand. (4) Pass the left hand above the right little-finger loop, hook the left little-finger into the left 
hand string of the right thumb loop and pull it across to the left hand. (5) Drop the right thumb loop and put the right thumb into the right little-finger loop. (6) Finally, withdraw both little-fingers, and separate the hands; the string will then come off the button-hole. In fact the effect of the movements described is to get both thumbs in one loop.

The Waistcoat Puzzle. The object is to enable a man wearing a coat and waistcoat of the usual patterns to remove the latter (which may be unbuttoned) without pulling it over his head or taking off his coat. Though here described in this way, it can be presented and is of the nature of a string trick.

The removal can be effected thus: (1) Take the left corner (or lappel) of the coat and push it through the left armhole of the waistcoat, from outside to inside. (2) Put the left hand and arm through the same armhole. The effect of this is to leave the left armhole of the waistcoat at the back of the neck. (3) Take the right lappel of the coat and put it through the left armhole of the waistcoat. (4) Put the right hand and arm through the same armhole. (5) Finally, pass the waistcoat down the right sleeve of the coat. The operation tends to injure the set of a good garment, and I suggest that a dressing or flannel jacket may be advantageously substituted for the coat. 


\section{CHAPTER XVI.}

THREE OLASSTCAL GEOMETRICAL PROBLEMS.

Among the more interesting geometrical problems of antiquity are three questions which attracted the special attention of the early Greek mathematicians. Our knowledge of geometry is derived from Greek sources, and thus these questions have attained a classical position in the history of the subject. The three questions to which I refer are (i) the duplication of a cube, that is, the determination of the side of a cube whose volume is double that of a given cube; (ii) the trisection of an angle; and (iii) the squaring of a circle, that is, the determination of a square whose area is equal to that of a given circle-each problem to be solved by a geometrical construction involving the use of straight lines and circles only, that is, by Euclidean geometry.

This limitation to the use of straight lines and circles implies that the only instruments available in Euclidean geometry are compasses and rulers. But the compasses must be capable of opening as wide as is desired, and the ruler must be of unlimited length. Further the ruler must not be graduated, for if there were two fixed marks on it we can obtain construstions equivalent to those obtained by the use of the conic sections.

With the Euclidean restriction all three problems are insoluble*. To duplicate a cube the length of whose side is $a$,

- See F. C. Klein, Vorträge über ausgeroühlte Fragen der Elementargeometrie, Leipzig, 1895; and F. G. Texeira, Sur les Problèmes célèbres de la Geométrie Élementaire non resolubles avec la Règle et le Compas, Coimbra, 1915. It is said that the earliest rigorous proof that the problems were insoluble by Euclidean geometry was giren by P. L. Wantzell in 1837 .

B. $\mathrm{R}$. 
we have to find a line of length $x$, such that $x^{s}=2 a^{s}$. Again, to trisect a given angle, we may proceed to find the sine of the angle, say $a$, then, if $x$ is the sine of an angle equal to one-third of the given angle, we have $4 x^{3}=3 x-a$. Thus the first and second problems, when considered analytically, require the solution of a cubic equation; and since a cohstruction by means of circles (whose equations are of the form $x^{2}+y^{2}+a x+b y+c=0$ ) and straight lines (whose equations are of the form $\alpha x+\beta y+\gamma=0$ ) cannot be equivalent to the solution of a cubic equation, it is inferred that the problems are insoluble if in our constructions we are restricted to the use of circles and straight lines. If the use of the conic sections is permitted, both of these questions can be solved in many ways. The third problem is different in character, but under the same restrictions it also is insoluble.

I propose to give some of the constructions which have been proposed for solving the first two of these problems. To save space I shall not draw the necessary diagrams, and in most cases I shall not add the proofs: the latter present but little difficulty. I shall conclude with some historical notes on approximate solutions of the quadrature of the circle.

\section{The Duplication of the Cube*.}

The problem of the duplication of the cube was known in ancient times as the Delian problem, in consequence of a legend that the Delians had consulted Plato on the subject. In one form of the story, which is related by Philoponust, it is asserted that the Athenians in 430 B.C., when suffering from the plague of eruptive typhoid fever, consulted the oracle at Delos as to how they could stop it. Apollo replied that they must double the size of his altar which was in the form of a cube. To the unlearned suppliants nothing seemed more easy, and a new altar was constructed either having each of its edges

* See Historia Problematis de Cubi Duplicatione by N. T. Reimer, Göttingen, 1798; and Historia Problematis Cubi Duplicandi by C. H. Biering, Copenhagen; 1844: also Das Delische Problem, by A. Sturm, Linz, 1895-7. Some notes on the subject are given in my History of Mathematics.

+ Philoponus ad Aristotelis Analytica Posteriora, bk. I, ohap. vii. 
double that of the old one (from which it followed that the volume was increased eight-fold) or by placing a similar cube altar next to the old one. Whereupon, according to the legend, the indignant god made the pestilence worse than before, and informed a fresh deputation that it was useless to trifle with him, as his new altar must be a cube and have a volume exactly double that of his old one. Suspecting a mystery the Athenians applied to Plato, who referred them to the geometricians. The insertion of Plato's name is an obvious anachronism. Eratosthenes* relates a somewhat similar story, but with Minos as the propounder of the problem.

In an Arab work, the Greek legend was distorted into the following extraordinarily impossible piece of history, which I cite as a curiosity of its kind. "Now in the days of Plato," says the writer, "a plague broke out among the children of Israel. Then came a voice from heaven to one of their prophets, saying, 'Let the size of the cubic altar be doubled, and the plague will cease'; so the people made another altar like unto the former, and laid the same by its side. Nevertheless the pestilence continued to increase. And again the voice spake unto the prophet, saying, "They have made a second altar like unto the former, and laid it by its side, but that does not produce the duplication of the cube.' Then applied they to Plato, the Grecian sage, who spake to them, saying, "Ye have been neglectful of the science of geometry, and therefore hath God chastised you, since geometry is the most sublime of all the sciences.' Now, the duplication of a cube depends on a rare problem in geometry, namely...." And then follows the solution of Apollonius, which is given later.

If $a$ is the length of the side of the given cube and $x$ that of the required cube, we have $x^{3}=2 a^{8}$, that is, $x: a=\sqrt[8]{ } 2: 1$. It is probable that the Greeks were aware that the latter ratio is incommensurable, in other words, that no two integers can be found whose ratio is the same as that of $\sqrt[3]{2}: 1$, but it did not therefore follow that they could not find the ratio by

- Archimedis Opera cum Eutocii Comnentariis, ed. Torelli, Oxford, 1792, p. 144 ; ed. Heiberg, Leipzig, 1880-1, vol, III, pp. 104-107. 
geometry: in fact, the side and diagonal of a square are instances of lines whose numerical measures are incommensurable.

I proceed now to give some of the geometrical constructions which have been proposed for the duplication of the cube* With one exception, I confine myself to those which can be effected by the aid of the conic sections.

Hippocrates $\nmid$ (circ. 420 B.c.) was perhaps the earliest mathematician who made any progress towards solving the problem. He did not give a geometrical construction, but he reduced the question to that of finding two means between one straight line $(a)$, and another twice as long (2a). If these means are $x$ and $y$, we have $a: x=x: y=y: 2 a$, from which it follows that $x^{3}=2 a^{3}$. It is in this form that the problem is always presented now. Formerly any process of solution by finding these means was called a mesolabum.

One of the first solutions of the problem was that given by Archytass in or about the year 400 B.c. His construction is equivalent to the following. On the diameter $O A$ of the base of a right circular cylinder describe a semicircle whose plane is perpendicular to the base of the cylinder. Let the plane containing this semicircle rotate round the generator through 0 , then the surface traced out by the semicircle will cut the cylinder in a tortuous curve. This curve will itself be cut by a right cone, whose axis is $O A$ and semi-vertical angle is (say) $60^{\circ}$, in a point $P$, such that the projection of $O P$ on the base of the cylinder will be to the radius of the cylinder in the ratio of the side of the required cube to that of the given cube. Of course the proof given by Archytas is geometrical; and it is interesting to note that in it he shows himself familiar with the results of the propositions Euc. III, 18, III, 35, and XI, 19. To

* On the application to this problem of the traditional Greek methods of analysis by Hero and Philo (leading to the solution by the use of Apollonius's circle), by Nicomedis (leading to the solution by the use of the conchoid), and by Pappus (leading to the solution by the use of the cissoid), see Geometrical Analysis by J. Leslie, Edinburgh, second edition, 1811, pp. 247-250, 453.

$\dagger$ Proclus, ed. Friedlein, pp. 212-213.

$\ddagger$ Archimedis Opera, ed. Torelli, p. 143; ed. Heiberg, rol. II1, pp. 98-103. 
show analyticilly that the construction is correct, take $O A$ as the axis of $x$, and the generator of the cylinder drawn through 0 as axis of $z$, then with the usual notation, in polar co-ordinates, if $a$ is the radius of the cylinder, we have for the equation of the surface described by the semicircle $r=2 a \sin \theta$; for that of the cylinder $r \sin \theta=2 \alpha \cos \phi$; and for that of the cone $\sin \theta \cos \phi=\frac{1}{2}$. These three surfaces cut in a point such that $\sin ^{3} \theta=\frac{1}{2}$, and therefore $(r \sin \theta)^{3}=2 a^{3}$. Hence the volume of the cube whose side is $r \sin \theta$ is twice that of the cube whose side is $a$.

The construction attributed to Plato* (circ. 360 B.c.) depends on the theorem that, if $C A B$ and $D A B$ are two rightangled triangles, having one side, $A B$, common, their other sides, $A D$ and $B C$, parallel, and their hypothenuses, $A C$ and $B D$, at right angles, then if these hypothenuses cut in $P$, we have $P C: P B=P B: P A=P A: P D$. Hence, if such a figure can be constructed having $P D=2 P C$, the problem will be solved. It is easy to make an instrument by which the figure can be drawn.

The next writer whose name is connected with the problem is Menaechmust, who in or about 340 B.c. gave two solutions of it.

In the first of these he pointed out that two parabolas having a common vertex, axes at right angles, and such that the latus rectum of the one is double that of the other, will intersect in another point whose abscissa (or ordinate) will give a solution. If we use analysis this is obvious; for, if the equations of the parabolas are $y^{2}=2 a x$ and $x^{2}=a y$, they intersect in a point whose abscissa is given by $x^{3}=2 a^{3}$. It is probable that this method was suggested by the form in which Hippocrates had cast the problem: namely, to find $x$ and $y$ so that $a: x=x: y=y: 2 a$, whence we have $x^{2}=a y$ and $y^{2}=2 a x$.

The second solution given by Menaechmus was as follows. Describe a parabola of latus rectum $l$. Next describe a rectangular hyperbola, the length of whose real axis is $4 l$, and

* Archimedis Opera, ed. Torelli, p. 135; ed. Heiberg, vol. III, pp. 66-71.

$\uparrow$ Ibid., ed. Torelli, pp. 141-143; ed. Heiberg, vol. rII, pp. 92-99. 
having for its asymptotes the tangent at the vertex of the parabola and the axis of the parabola. Then the ordinate and the abscissa of the point of intersection of these curves are the mean proportionals between $l$ and $2 l$. This is at once obvious by analysis. The curves are $x^{2}=l y$ and $x y=2 l^{2}$. These cut in a point determined by $x^{3}=2 l^{3}$ and $y^{3}=4 l^{3}$. Hence

$$
l: x=x: y=y: 2 l \text {. }
$$

The solution of Apollonius*, which was given about 220 B.C., was as follows. The problem is to find two mean proportionals between two given lines. Construct a rectangle $O A D B$, of which the adjacent sides $O A$ and $O B$ are respectively equal to the two given lines. Bisect $A B$ in $C$. With $C$ as centre describe a circle cutting $O A$ produced in $a$ and cutting $O B$ produced in $b$, so that $a D b$ shall be a straight line. If this circle can be so described, it will follow that $O A: B b=B b: A a=A a: O B$, that is, $B b$ and $A a$ are the two mean proportionals between $O A$ and $O B$. It is impossible to construct the circle by Euclidean geometry, but Apollonius gave a mechanical way of describing it.

The only other construction of antiquity to which $\mathbf{I}$ will refer is that given by Diocles and Sporus†. It is as follows. Take two sides of a rectangle $O A, O B$, equal to the two lines between which the means are sought. Suppose $O A$ to be the greater. With centre $O$ and radius $O A$ describe a circle. Let $O B$ produced cut the circumference in $C$ and let $A O$ produced cut it in $D$. Find a point $E$ on $B C$ so that if $D E$ cuts $A B$ produced in $F$ and cuts the circumference in $G$, then $F E=E G$. If $E$ can be found, then $O E$ is the first of the means between $O A$ and $O B$. Diocles invented the cissoid in order to determine $E$, but it can be found equally conveniently by the aid of conics.

In more modern times several other solutions have been suggested. I may allude in passing to three given by Huygenst,

* Archimedis Opera, ed. Torelli, p. 137; ed. Heiberg, vol, III, pp. 76-79. The solution is given in my History of Mathematics, London, 1901, p. $8 \pm$.

+ Ibid., ed. Torelli, pp. 138, 139, 141; ed. Heiberg, vol. III, pp. 78-84, 90-93.

$\ddagger$ Opera Varia, Leyden, 1724, pp. 393-396. 
but I will enunciate only those proposed respectively by Vieta, Descartes, Gregory of St Vincent, and Newton.

Vieta's construction is as follows*. Describe a circle, centre $O$, whose radius is equal to half the length of the larger of the two given lines. In it draw a chord $A B$ equal to the smaller of the two given lines. Produce $A B$ to $E$ so that $B E=A B$. Through $A$ draw a line $A F$ parallel to $O E$. Through $O$ draw a line $D O C F G$, cutting the circumference in $D$ and $C$, cutting $A F$ in $F^{\prime}$, and cutting $B A$ produced in $G$, so that $G F=O A$. If this line can be drawn then $A B: G C=G C: G A=G A: C D$.

Descartes pointed out $\nmid$ that the curves

$$
x^{2}=a y \text { and } x^{2}+y^{2}=a y+b x
$$

cut in a point $(x, y)$ such that $a: x=x: y=y: b$. Of course this is equivalent to the first solution given by Menaechmus, but Descartes preferred to use a circle rather than a second conic.

Gregory's construction was given in the form of the following theoremt. The hyperbola drawn through the point of intersection of two sides of a rectangle so as to have the two other sides for its asymptotes meets the circle circumscribing the rectangle in a point whose distances from the asymptotes are the mean proportionals between two adjacent sides of the rectangle. This is the geometrical expression of the proposition that the curves $x y=a b$ and $x^{2}+y^{2}=a y+b x$ cut in a point $(x, y)$ such that $a: x=x: y=y: b$.

One of the constructions proposed by Newton is as follows Let $O A$ be the greater of two given lines. Bisect $O A$ in $B$. With centre $O$ and radius $O B$ describe a circle. Take a point $C$ on the circumference so that $B C$ is equal to the other of the two given lines. From $O$ draw $O D E$ cutting $A C$ produced in $D$, and $B C$ produced in $E$, so that the intercept $D E=O B$. Then

* Opera Mathematica, ed. Schooten, Leyden, 1646, prop. v, pp. 242-243.

+ Geometria, bk. III, ed. Schooten, Amsterdam, 1659, p. 91.

¥ Gregory of St Vincent, Opus Geometricum Quadraturae Circuli, Antwerp, 1647 , bk. VI, prop. 138, p. 602 .

$\$$ Arithmetica Universalis, Ralphson's (second) edition, 1728, p. 242; see siso pp. $243,245$. 
$B C: O D=O D: C E=C E: O A$. Hence $O D$ and $C E$ are two mean proportionals between any two lines $B C$ and $O A$.

\section{The Trisection of an Angle*.}

The trisection of an angle is the second of these classical problems, but tradition has not enshrined its origin in romance. The following two constructions are among the oldest and best known of those which have been suggested; they are quoted by Pappust, but I do not know to whom they were due originally.

The first of them is as follows. Let $A O B$ be the given angle. From any point $P$ in $O B$ draw $P M$ perpendicular to $O A$. Through $P$ draw $P R$ parallel to $O A$. On $M P$ take a point $Q$ so that if $O Q$ is produced to cut $P R$ in $R$ then $Q R=2 . O P$. If this construction can be made, then $A O R=\frac{1}{3} A O B$. The solution depends on determining the position of $R$. This was effected by a construction which may be expressed analytically thus. Let the given angle be $\tan ^{-1}(b / a)$. Construct the hyperbola $x y=a b$, and the circle $(x-a)^{2}+(y-b)^{2}=4\left(a^{2}+b^{2}\right)$. Of the points where they cut, let $x$ be the abscissa which is greatest, then $P R=x-a$, and $\tan ^{-1}(b / x)=\frac{1}{3} \tan ^{-1}(b / a)$.

The second construction is as follows. Let $A O B$ be the given angle. Take $O B=O A$, and with centre $O$ and radius $O A$ describe a circle. Produce $A O$ indefinitely and take a point $C$ on it external to the circle so that if $C B$ cuts the circumference in $D$ then $C D$ shall be equal to $O A$. Draw $O E$ parallel to $C D B$. Then, if this construction can be made, $A O E=\frac{1}{3} A \cup B$. The ancients determined the position of the point $C$ by the aid of the conchoid: it could be also found by the use of the conic sections.

I proceed to give a few other solutions, confining myself to those effected by the aid of conics.

* On the bibliography of the subject see the supplements to L'Intermediaire des Mathématiciens, Paris, May and June, 1904.

+ Pappus, Mathematicae Collectiones, bk. Iv, props. 32, 33 (ed. Commandino, Bonn, 1670, pp. 97-99). On the application to this problem of the traditional Greek methods of analysis seo Geometrical Analysis, by J. Leslie, Edinburgh, second edition, 1811, pp. 245-247. 
Among other constructions given by Pappus* I may quote the following. Describe a hyperbola whose eccentricity is two. Let its centre be $C$ and its vertices $A$ and $A^{\prime}$. Produce $C A^{\prime}$ to $S$ so that $A^{\prime} S=C A^{\prime}$. On $A S$ describe a segment of a circle to contain the given angle. Let the orthogonal bisector of $A S$ cut this segment in $O$. With centre $O$ and radius $O A$ or $O S$ describe a circle. Let this circle cut the branch of the hyperbola through $A^{\prime}$ in $P$. Then $S O P=\frac{1}{3} S O A$.

In modern times one of the earliest of the solutions by a direct use of conics was suggested by Descartes, who effected it by the intersection of a circle and a parabola. His construction $\dagger$ is equivalent to finding the points of intersection, other than the origin, of the parabola $y^{2}=\frac{1}{4} x$ and the circle. $x^{2}+y^{2}-\frac{18}{4} x+4 a y=0$. The ordinates of these points are given by the equation $4 y^{3}=3 y-a$. The smaller positive root is the sine of one-third of the angle whose sine is $a$. The demonstration is ingenious.

One of the solutions proposed by Newton is practically equivalent to the third one which is quoted above from Pappus. It is as followst. Let $A$ be the vertex of one branch of a hyperbola whose eccentricity is two, and let $S$ be the focus of the other branch. On $A S$ describe the segment of a circle containing an angle equal to the supplement of the given angle. Let this circle cut the $S$ branch of the hyperbola in $P$. Then $P A S$ will be equal to one-third of the given angle.

The following elegant solution is due to Clairauţ. Let $A O B$ be the given angle, Take $O A=O B$, and with centre $O$ and radius $O A$ describe a circle. Join $A B$, and trisect it in $H, K$, so that $A H=H K=K B$. Bisect the angle $A O B$ by $O C$ cutting $A B$ in $L$. Then $A H=2 . H L$. With focus $A$, vertex $H$, and directrix $O C$, describe a hyperbola. Let the branch of

- Pappus, Mathematicae Collectiones, bk. IV, prop. 34, pp. 99-104.

+ Geometria, bk. III, ed. Schooten, Amsterdam, 1659, p. 91.

$¥$ Arithmetica Universalis, problem xuII, Ralphson's (second) edition, London, 1728 , p. 148 ; see also pp. $243-245$.

$\S$ I believe that this was first given by Clairaut, but I have mislaid my reference. The construction occurs as an example in the Geometry of Conics, by C. Taylor, Cambridge, 1881, No. 308, p. 126. 
this hyperbola which passes through $B$ cut the circle in $P$. Draw $P M$ perpendicular to $O C$ and produce it to cut the circle in $Q$. Then by the focus and directrix property we have $A P: P M=A H: H L=2: 1, \therefore A P=2 . P M=P Q$. Hence, by symmetry, $A P=P Q=Q R . \quad \therefore A O P=P O Q=Q O R$.

I may conclude by giving the solution which Chasles* regards as the most fundamental. It is equivalent to the following proposition. If $O A$ and $O B$ are the bounding radii of a circular arc $A B$, then a rectangular hyperbola having $O A$ for a diameter and passing through the point of intersection of $O B$ with the tangent to the circle at $A$ will pass through one of the two points of trisection of the arc.

Several instruments have been constructed by which mechanical solutions of the problem can be obtained.

\section{The Quadrature of the Circle†.}

The object of the third of the classical problems was the determination of a side of a square whose area should be equal to that of a given circle.

The investigation, previous to the last two hundred years, of this question was fruitful in discoveries of allied theorems, but in more recent times it has been abandoned by those who are able to realize what is required. The history of this subject has been treated by competent writers in such detail that I shall content myself with a very brief allusion to it.

Archimedes showed + (what possibly was known before) that the problem is equivalent to finding the area of a right-angled

- Traite des sections coniques, Paris, 1865, art. 37, p. 36.

+ See Montucla's Histoire des Recherches sur la Quadrature du Cercle, edited by P. L. Lacroix, Paris, 1831; also various articles by A. Do Morgan, and especially his Budget of Paradoxes, London, 1872. A popular sketoh of the subject has been compiled by H. Schubert, Die Quadratur des Zirkels, Hamburg, 1889; and since the publication of the earlier editions of these Recreations Prof. F. Rudio of Zurich has given an analysis of the arguments of Archimedes, Huygens, Lambert, and Legendre on the subject, with an introduction on the history of the problem, Leipzig, 1892.

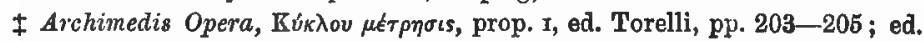
Heiberg, vol. I, pp. 258-261, vol. III, pp. 269-277. 
triangle whose sides are equal respectively to the perimeter of the circle and the radius of the circle. Half the ratio of these lines is a number, usually denoted by $\pi$.

That this number is incommensurable had been long suspected, and has been now demonstrated. The earliest analytical proof of it was given by Lambert* in 1761; in 1803 Legendre $†$ extended the proof to show that $\pi^{2}$ was also incommensurable; and recently Lindemann + has shown that $\pi$ sannot be the root of a rational algebraical equation.

An earlier attempt. by James Gregory to give a geometrical demonstration of this is worthy of notice. Gregory proved $\S$ that the ratio of the area of any arbitrary sector to that of the inscribed or circumscribed polygons is not expressible by a finite number of algebraical terms. Hence he inferred that the quadrature was impossible. This was accepted by Montucla, but it is not conclusive, for it is conceivable that some particular sector might be squared, and this particular sector might be the whole circle.

In connection with Gregory's proposition above cited, I may add that Newton $\|$ proved that in any closed oval an arbitrary sector bounded by the curve and two radii cannot be expressed in terms of the co-ordinates of the extremities of the arc by a finite number of algebraical terms. The argument is condensed and difficult to follow: the same reasoning would show that a closed oval curve cannot be represented by an algebraical equation in polar co-ordinates. From this proposition no

* Mémoires de l'Acadêmie de Berlin for 1761, Berlin, 1768, pp. 265322.

† Legendre's Geometry, Brewster's translation, Edinburgh, 1824, pp. 239245.

$\ddagger$ Ueber die Zahl $\pi$, Mathematische Annalen, Leipzig, 1882, vol. xx, pp. 213225. The proof leads to the conclusion that, if $x$ is a root of a rational integral algebraical equation, then $e^{x}$ cannot be rational : hence, if $\pi i$ was the root of such an equation, $e^{\pi i}$ could not be rational; but $e^{\pi i}$ is equal to -1 , and therefore is rational; henee $\pi i$ cannot be the root of suoh an algebraical equation, and therefore neither can $\pi$.

\& Vera Circuli ct Hyperbolae Quadratura, Padua, 1668: this is reprinted in Huygens's Opera Varia, Leyden, 1724, pp. 405-462.

|l Principia, bk. I, section vi, lemma sxviu. 
conclusion as to the quadrature of the circle is to be drawn, nor did Newton draw any. In the earlier editions of this work I expressed an opinion that the result presupposed a particular definition of the word oval, but on more careful reflection I think that the conclusion is valid without restriction.

With the aid of the quadratrix, or the conchoid, or the cissoid, the quadrature of the circle is easy, but the construction of those curves assumes a knowledge of the value of $\pi$, and thus the question is begged.

I need hardly add that, if $\pi$ represented merely the ratio of the circumference of a circle to its diameter, the determination of its numerical value would have but slight interest. It is however a mere accident that $\pi$ is defined usually in that way, and it really represents a certain number which would enter into analysis from whatever side the subject was approached.

I recollect a distinguished professor explaining how different would be the ordinary life of a race of beings born, as easily they might be, so that the fundamental processes of arithmetic, algebra and geometry were different to those which seem to us so evident, but, he added, it is impossible to conceive of a universe in which $e$ and $\pi$ should not exist.

I have quoted elsewhere an anecdote, which perhaps will bear repetition, that illustrates how little the usual definition of $\pi$ suggests its properties. De Morgan was explaining to an actuary what was the chance that a certain proportion of some group of people would at the end of a given time be alive; and quoted the actuarial formula, involving $\pi$, which, in answer to a question, he explained stood for the ratio of the circumference of a circle to its diameter. His acquaintance, who had so far listened to the explanation with interest, interrupted him and exclaimed, "My dear friend, that must be a delusion, what can a circle have to do with the number of people alive at the end of a given time?" In reality the fact that the ratio of the length of the circumference of a circle to its diameter is the number denoted by $\pi$ does not afford the best analytical definition of $\pi$, and is only one of its properties. 
The use of a single symbol to denote this number $3 \cdot 14159 .$. seems to have been introduced about the beginning of the eighteenth century. William Jones * in 1706 represented it by $\pi$; a few years later $\dagger$ John Bernoulli denoted it by $c$; Euler in 1734 used $p$, and in 1736 used $c$; Christian Goldback in 1742 used $\pi$; and after the publication of Euler's Analysis the symbol $\pi$ was generally employed.

The numerical value of $\pi$ can be determined by either of two methods with as close an approximation to the truth as is desired.

The first of these methods is geometrical. It consists in calculating the perimeters of polygons inscribed in and circumscribed about a circle, and assuming that the circumference of the circle is intermediate between these perimeterst. The approximation would be closer if the areas and not the perimeters were employed. The second and modern method rests on the determination of converging infinite series for $\pi$.

We may say that the $\pi$-calculators who used the first method regarded $\pi$ as equivalent to a geometrical ratio, but those who adopted the modern method treated it as the symbol for a certain number which enters into numerous branches of mathematical analysis.

It may be interesting if I add here a list of some of the approximations to the value of $\pi$ given by various writers $\$$. This will indicate incidentally those who have studied the subject to the best advantage.

- Synopsis Palmariorum Matheseos, London, 1706, pp. 243, 263 et seq.

† See notes by G. Eneström in the Bibliotheca Mathematica, Stockholm, 1839, vol. III, p. 28 ; Ibid., 1890, vol. Iv, p. 22.

¥ The history of this method has been written by K. E. I. Selander, Historik ofver Ludolphska Talet, Upsala, 1868.

$\S$ For the methods used in classical times and the results obtained, see the notices of their authors in M. Cantor's Geschichte der Mathematik, Leipzig, vol. I, 1880. For medieval and modern approximations, see the article by A. De Morgan on the Quadrature of the Circle in vol. XIX of the Penny Cyclopaedia, London, 1841; with the additions given by B. de Haan in the Verhandelingen of Amsterdam, 1858, vol. Iv, p. 22: the conclusions were tabulated, corrected, and extended by Dr J. W. L. Glaisher in the Messenger of Mathematics, Cambridge, 1873, vol. II, pp. 119-128; and Ibid., 1874, vol. Irr, pp. $27-46$. 
The ancient Egyptians * took 256/81 as the value of $\pi$, this is equal to $3 \cdot 1605 . .$. ; but the rougher approximation of $\mathbf{3}$ was used by the Babylonians $\uparrow$ and by the Jews.. It is not unlikely that these numbers were obtained empirically.

We come next to a long roll of Greek mathematicians who attacked the problem. Whether the researches of the members of the Ionian School, the Pythagoreans, Anaxagoras, Hippias, Antipho, and Bryso led to numerical approximations for the value of $\pi$ is doubtful, and their investigations need not detain us. The quadrature of certain lunes by Hippocrates of Chios is ingenious and correct, but a value of $\pi$ cannot be thence deduced; and it seems likely that the later members of the Athenian School concentrated their efforts on other questions.

It is probable that Euclid $\S$, the illustrious founder of the Alexandrian School, was aware that $\pi$ was greater than 3 and less than 4, but he did not state the result explicitly.

The mathematical treatment of the subject began with Archimedes, who proved that $\pi$ is less than $3 \frac{1}{7}$ and greater than $3 \frac{10}{7}$, that is, it lies between $3 \cdot 1428 \ldots$ and $3 \cdot 1408 \ldots \mathrm{He}$ established $\|$ this by inscribing in a circle and circumscribing about it regular polygons of 96 sides, then determining by geometry the perimeters of these polygons, and finally assuming that the circumference of the circle was intermediate between these perimeters : this leads to a result from which he deduced the limits given above. This method is equivalent to using the proposition $\sin \theta<\theta<\tan \theta$, where $\theta=\pi / 96$ : the values of $\sin \theta$ and $\tan \theta$ were deduced by Archimedes from those of $\sin \frac{1}{8} \pi$ and $\tan \frac{1}{3} \pi$ by repeated bisections of the angle. With a polygon of $n$ sides this

- Ein mathematisches Handluch der alten Aegypter (i.e. the Rhind papyrus), by A. Eisenlohr, Leipzig, 1877, arts. 100-109, 117, 124.

† Oppert, Journal Asiatique, August, 1872, and October, 1874.

\$ 1 Kings, ch. 7, ver. 23 ; 2 Chronioles, ch. 4, ver. 2.

\$ These results can be deduced from Euc. IV, 15, and IV, 8: see also book III, prop. 16.

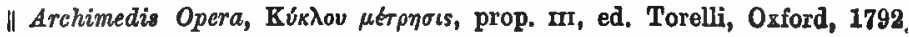
pp. 205-216; ed. Heiberg, Leipzig, 1880, vol. I, pp. 263-271. 
process gives a value of $\pi$ correct to at least the integral part of $(2 \log n-1 \cdot 19)$ places of decimals. The result given by Archimedes is correct to two places of decimals. His analysis leads to the conclusion that the perimeters of these polygons for a circle whose diameter is 4970 feet would lie between 15610 feet and 15620 feet-actually it is about 15613 feet 9 inches.

Apollonius discussed these results, but his criticisms have been lost.

Hero of Alexandria gave the value 3, but he quoted the result 22/7 : possibly the former number was intended only for rough approximations.

The only other Greek approximation that I need mention is that given by Ptolemy+, who asserted that $\pi=3^{\circ} 8^{\prime} 30^{\prime \prime}$. This is equivalent to taking $\pi=3+\frac{8}{60}+\frac{80}{3600}=3 \frac{17}{12}=31141 \dot{6}$.

The Roman surveyors seem to have used 3, or sometimes 4 , for rough calculations. For closer approximations they often employed $3 \frac{1}{8}$ instead of $3 \frac{1}{7}$, since the fractions then introduced are more convenient in duodecimal arithmetic. On the other hand Gerbert $\$$ recommended the use of 22/7.

Before coming to the medieval and modern European mathematicians it may be convenient to note the results arrived at in India and the East.

Baudhayana|| took 49/16 as the value of $\pi$.

Arya-Bhata T, circ. 530, gave $62832 / 20000$, which is equal to 3:1416. He showed that, if $a$ is the side of a regular polygon of $n$ sides inscribed in a circle of unit diameter, and if $b$ is the side of a regular inscribed polygon of $2 n$ sides, then $b^{2}=\frac{1}{2}-\frac{1}{2}\left(1-a^{2}\right)^{\frac{1}{2}}$. From the side of an inscribed hexagon, he found successively the sides of polygons of $12,24,48,96,192$,

- Mensurae, ed. Hultsch, Berlin, 1864, p. 188.

+ Geometria, ed. Hultsch, Berlin, 1864, pp. 115, 136.

$\ddagger$ Almagest, bl. vi, chap. 7 ; ed. Halma, vol. I, p. 421.

$\$$ Euvres de Gerbert, ed. Olleris, Clermont, 1867, p. 453.

\| The Sulvasutras by G. Thibaut, Asiatic Society of Bengal, 1875, arts. 26-28.

If Leçons de calcul d'Aryabhata, by L. Bodet in the Journal Asiatique, 1879, series 7, vol. zIII, pp. 10, 21. 
and 384 sides. The perimeter of the last is given as equal to $\sqrt{9 \cdot 8694}$, from which his result was obtained by approximation.

Brahmagupta*, circ. 650 , gave $\sqrt{10}$, which is equal to $3 \cdot 1622 \ldots$ He is said to have obtained this value by inscribing in a circle of unit diameter regular polygons of $12,24,48$, and 96 sides, and calculating successively their perimeters, which he found to be $\sqrt{9 \cdot 65}, \sqrt{9 \cdot 81}, \sqrt{9 \cdot 86}, \sqrt{9 \cdot 87}$ respectively; and to . have assumed that as the number of sides is increased indefinitely the perimeter would approximate to $\sqrt{\mathbf{1 0}}$.

Bhaskara, circ. 1150, gave two approximations. One†-possibly copied from Arya-Bhata, but said to have been calculated afresh by Archimedes's method from the perimeters of regular polygons of 384 sides-is $3927 / 1250$, which is equal to $3 \cdot 1416$ : the other is $754 / 240$, which is equal to $3 \cdot 141 \dot{6}$, but it is uncertain whether this was not given only as an approximate value.

Among the Arabs the values $22 / 7, \sqrt{10}$, and $62832 / 20000$ were given by Alkarismi§, circ. 830 ; and no doubt were derived from Indian sources. He described the first as an approximate value, the second as used by geometricians, and the third as used by astronomers.

In Chinese works the values $3,22 / 7,157 / 50$ are said to occur. probably the last two results were copied from the Arabs. The Japanese\| approximations were closer.

Returning to European mathematicians, we have the following successive approximations to the value of $\pi$ : many of those prior to the eighteenth century having been calculated originally.with the view of demonstrating the incorrectness of some alleged quadrature.

* Algebra..from Brahmegupta and Bhascara, trans. by H. T. Colebrooke, London, 1817, chap. xıi, art. 40, p. 308.

+ Ibid., p. 87.

¥ Ibid., p. 95 .

\$ The Algebra of Mohammed ben Musa, ed. by F. Rosen, London, 1831, pp. $71-72$.

|| On Japanese approximations and the methods used, see P. Harzer, Transactions of the British Association for 1905, p. 325. 
Leonardo of Pisa*, in the thirteenth century, gave for $\pi$ the value $1440 / 458 \frac{1}{3}$, which is equal to $3 \cdot 1418 \ldots$ In the fifteenth century, Purbach $\dagger$ gave or quoted the value 62832/20000, which is equal to $3 \cdot 1416$; Cusa believed that the accurate value was $\frac{3}{4}(\sqrt{ } 3+\sqrt{ } 6)$, which is equal to $3 \cdot 1423 \ldots$; and, in 1464 , Regiomontanus + is said to have given a value equal to $3 \cdot 14243$.

Vieta \$, in 1579 , showed that $\pi$ was greater than $31415926535 / 10^{10}$, and less than $31415926537 / 10^{10}$. This was deduced from the perimeters of the inscribed and circumscribed polygons of $6 \times 2^{16}$ sides, obtained by repeated use of the formula $2 \sin ^{2} \frac{1}{2} \theta=1-\cos \theta$. He also gave $\|$ a result equivalent to the formula

$$
\frac{2}{\pi}=\frac{\sqrt{ } 2}{2} \frac{\sqrt{ }(2+\sqrt{ } 2)}{2} \frac{\sqrt{ }\{2+\sqrt{ }(2+\sqrt{ } 2)\}}{2} \ldots .
$$

The father of Adrian Metius T, in 1585, gave 355/113, which is equal to $3 \cdot 14159292 \ldots$, and is correct to six places of decimals. This was a curious and lucky guess, for all that he proved was that $\pi$ was intermediate between $377 / 120$ and $333 / 106$, whereon he jumped to the conclusion that he would obtain the true fractional value by taking the mean of the numerators and the mean of the denominators of these fractions.

In 1593 Adrian Romanus** calculated the perimeter of the inscribed regular polygon of 1073,741824 (i.e. $2^{30}$ ) sides, from which he determined the value of $\pi$ correct to 15 places of decimals.

* Boncompagni's Scritti di Leonardo, vol. II (Practica Geometriae), Rome, 1862, p. 90.

+ Appendix to the De Triangulis of Regiomontanus, Basle, 1541, p. 131.

* In his correspondence with Cardinal Nicholas de Cusa, De Quadratura Circuli, Nuremberg, 1533, wherein he proved that the cardinal's result was wrong. I cannot quote the exact reference, but the figures are given by competent writers and I have no doubt are correct.

\$ Canon Mathematicus seu ad Triangula, Paris, 1579, pp. 56, 66: probably this work was printed for private circulation only, it is very rare.

॥ Vietae Opera, ed. Schooten, Leyden, 1646, p. 400.

I Arithmeticae libri duo et Geometriae, by A. Metius, Leyden, 1626, pp. 8889. [Probably issued originally in 1611.]

* Ideae Mathematicae, Antwerp, 1593: a rare work, which I bave never been able to consult.

B. $\boldsymbol{n}$. 
L. van Ceulen devoted no inconsiderable part of his life to the subject. In $1596^{*}$ he gave the result to 20 places of decimals: this was calculated by finding the perimeters of the inscribed and circumscribed regular polygons of $60 \times 2^{3 s}$ sides, obtained by the repeated use of a theorem of his discovery equivalent to the formula $1-\cos A=2 \sin ^{2} \frac{1}{2} A$. I possess a finely executed engraving of him of this date, with the result printed round a circle which is below his portrait. He died in 1610 , and by his directions the result to 35 places of decimals (which was as far as he had calculated it) was engraved on his tombstone + in St Peter's Church, Leyden. His posthumous srithmetic + contains the result to 32 places; this was obtained by calculating the perimeter of a polygon, the number of whose sides is $2^{63}$, i.e. $4,611686,018427,387904$. Van Ceulen also compiled a table of the perimeters of various regular polygons.

Willebrord Snell sides an approximation to 34 places of decimals. This is less than the numbers given by van Ceulen, but Snell's method was so superior that he obtained his 34 places by the use of a polygon from which van Ceulen had obtained only 14 (or perhaps 16) places. Similarly, Snell obtained from a hexagon an approximation as correct as that for which Archimedes had required a polygon of 96 sides, while from a polygon of 96 sides he determined the value of $\pi$ correct to seven decimal places instead of the two places obtained by Archimedes. The reason is that Archimedes, having calculated the lengths of the sides of inscribed and circumscribed regular polygons of $n$ sides, assumed that the length of $1 / n$th of the perimeter of the circle was intermediate between them; whereas Snell constructed

*Vanden Circkel, Delf, 1596, fol.14, p. 1 ; or De Circulo, Leyden, 1619, p. 3.

† The inscription is quoted by Prof. de B.aan in the Messenger of Mathematics, 1874, vol. III, p. 25.

† De Arithmetische en Geometrische Fondamenten, Leyden, 1615, p. 163; or p. 144 of the Latin translation by W. Snell, published at Leyden in 1615 under the title Fundamenta Arithmetica et Geometrica. This was reissued, together with a Latin translation of the Vanden Circkel, in 1619, under the title De Circulo; in whioh see pp. 8, 29-32, 92.

8 Cyclometricus, Leyden, 1621, p. 55. 
from the sides of these polygons two other lines which gave closer limits for the corresponding arc. His method depends on the theorem $3 \sin \theta /(2+\cos \theta)<\theta<\left(2 \sin \frac{1}{3} \theta+\tan \frac{1}{3} \theta\right)$, by the aid of which a polygon of $n$ sides gives a value of $\pi$ correct to at least the integral part of $(4 \log n-2305)$ places of decimals, which is more than twice the number given by the older rule. Snell's proof of his theorem is incorrect, though the result is true.

Snell also added a table* of the perimeters of all regular inscribed and circumscribed polygons, the number of whose sides is $10 \times 2^{n}$ where $n$ is not greater than 19 and not less than 3. Most of these were quoted from van Ceulen, but some were recalculated. This list has proved useful in refuting circlesquares. A similar list was given by James Gregory †.

In 1630 Grienbergert, by the aid of Snell's theorem, carried the approximation to 39 places of decimals. He was the last mathematician who adopted the classical method of finding the perimeters of inscribed and circumscribed polygons. Closer approximations serve no useful purpose. Proofs of the theorems used by Snell and other calculators in applying this method were given by Huygens in a work $\S$ which may be taken as closing the history of this method.

In 1656 Wallis $\|$ proved that

$$
\frac{\pi}{2}=\frac{2 \cdot 2 \cdot 4 \cdot 4 \cdot 6 \cdot 6 \ldots}{1 \cdot 3 \cdot 3 \cdot 5 \cdot 5 \cdot 7 \cdot 7 \ldots}
$$

and quoted a proposition given a few years earlier by Viscount Brouncker to the effect that

$$
\frac{4}{\pi}=1+\frac{1^{2}}{2}+\frac{3^{2}}{2}+\frac{5^{2}}{2}+\ldots,
$$

- It is quoted by Montucla, ed. 1831, p. 70.

+ Vera Circuli et Hyperbolae Quadratura, prop. 29, quoted by Huygens, Opera Varia, Leyden, 1724, p. 447.

$\ddagger$ Elementa Trigonometrica, Rome, 1630, end of preface.

\$ De Circula Magnitudine Inventa, 1654; Opera Varia, pp. 351-387. The proofs are given in G. Pirie's Geometrical Methods of Approximating to the Value of $\pi$, London, 1877, pp. 21-23.

II Arithmetica Infinitomum, Oxford, 1656, prop. 191. An analysis of the investigation by Wallis was given by Cayloy, Quarterly Journal of Mathematics, 1889, vol. xxIII, pp. 165-169. 
but neither of these theorems was used to any large extent for calculation.

Subsequent calculators have relied on converging infinite series, a method that was hardly practicable prior to the invention of the calculus, though Descartes* had indicated a geometrical process which was equivalent to the use of such a series. The employment of infinite series was proposed by James Gregory $\uparrow$, who established the theorem that

$$
\theta=\tan \theta-\frac{1}{3} \tan ^{8} \theta+\frac{1}{6} \tan ^{5} \theta-\ldots,
$$

the result being true only if $\theta$ lies between $-\frac{1}{4} \pi$ and $\frac{1}{4} \pi$.

The first mathematician to make use of Gregory's series for obtaining an approximation to the value of $\pi$ was Abraham Sharp ${ }_{+}^{+}$, who, in 1699, on the suggestion of Halley, determined it to 72 places of decimals (71 correct). He obtained this value by putting $\theta=\frac{1}{6} \pi$ in Gregory's series.

Machin§, earlier than 1706, gave the result to 100 places (all correct). He calculated it by the formula

$$
\frac{1}{4} \pi=4 \tan ^{-1} \frac{1}{8}-\tan ^{-1} \frac{1}{238} \text {. }
$$

De Lagny\|, in 1719, gave the result to 127 places of decimals (112 correct), calculating it by putting $\theta=\frac{1}{8} \pi$ in Gregory's series.

Hutton $\%$, in 1776, and Euler**, in 1779, suggested the use of

- See Euler's paper in the Novi Commentarii Academiae Scientiarum, Petrograd, 1763, vol. vir, pp. 157-168.

+ See the letter to Collins, dated F'eb. 15, 1671, printed in the Commercium Epistolicum, London, 1712, p. 25, and in the Macclesfield Collection, Correspondence of Scientific Men of the Seventeenth Century, Oxford, 1841, vol. II, p. 216.

‡ See Life of A. Sharp by W. Cudworth, London, 1889, p. 170. Sharp's work is given in one of the preliminary discourses (p. 53 et seq.) prefixed to H. Sherwin's Mathematical Tables. The tables were issued at London in 1705: probably the discourses were issued at the same time, though the earliest copies I have seen were printed in 1717.

§ W. Jones's Synopsis Palmarionum, London, 1706, p. 243; and Maseres, Scriptores Logarithmici, London, 1796, vol. II, pp. vii-ix, 155-164.

\| Histoire de '`Académie for 1719, Paris, 1721, p. 144.

ๆ Thilosophical Transactions, 1776, vol. Lxvr, pp. 476-492.

** Nova Acta Academiae Scientiarum Petropolitanae for 1793, Petrograd, 1798, vol, xI, pp. 133-149: the memoix was read in 1779. 
the formula $\frac{1}{4} \pi=\tan ^{-1} \frac{1}{2}+\tan ^{-1} \frac{1}{3}$ or $\frac{1}{4} \pi=5 \tan ^{-1} \frac{1}{7}+2 \tan ^{-1} \frac{3}{79}$, but neither carried the approximation as far as had been done previously.

Vega, in $1789^{*}$, gave the value of $\pi$ to 143 places of decimals (126 correct); and, in 1794 †, to 140 places (136 correct).

Towards the end of the eighteenth century F. X. von Zach saw in the Radcliffe Library, Oxford, a manuscript by an unknown author which gives the value of $\pi$ to 154 places of decimals (152 correct).

In 1837, the result of a calculation of $\pi$ to 154 places of decimals (152 correct) was published + .

In 1841 Rutherford $\S$ calculated it to 208 places of decimals (152 correct), using the formula $\frac{1}{4} \pi=4 \tan ^{-1} \frac{1}{5}-\tan ^{-1} \frac{1}{70}+\tan ^{-1} \frac{1}{9 g}$.

In 1844, Dase $\|$ calculated it to 205 places of decimals (200) correct), using the formula $\frac{1}{4} \pi=\tan ^{-1} \frac{1}{2}+\tan ^{-1} \frac{1}{6}+\tan ^{-1} \frac{1}{8}$.

In 1847 Clausen $\uparrow$ carried the approximation to 250 places of decimals (248 correct), calculating it independently by the formulae $\frac{1}{4} \pi=2 \tan ^{-1} \frac{1}{3}+\tan ^{-1} \frac{1}{4}$ and $\frac{1}{4} \pi=4 \tan ^{-1} \frac{1}{6}-\tan ^{-1} \frac{1}{239}$.

In 1853 Rutherford** carried his former approximation to 440 places of decimals (all correct), and William Shanks prolonged the approximation to 530 places. In the same year Shanks published an approximation to 607 places $\nmid \dagger$ : and in 1873 he carried the approximation to 707 places of decimals ++. These were calculated from Machin's formula.

In 1853 Richter, presumably in ignorance of what had been

* Nova Acta Academiae Scientiarum Retropolitanae for 1790, Petrograd, 1795, vol. IX, p. 41.

† Thesaurus Logarithmorum (logarithmiseh-trigonometrischer Tafeln), Leipzig, 1794, p. 633.

¥J. F. Callet's Tables, etc., Précis Élementaire, Paris, tirage, 1837. Tirage, 1894, p. 96.

§ Philosophical Transactions, 1841, p. 283.

\| Crelle's Journal, 1844, vol, Exvir, p. 198.

TI Schumacher, Astronomische Nachrichtent, vol. $\mathbf{x} \nabla \mathbf{v}$, col. 207.

* Proceedings of the Royal Society, Jan. 20, 1853, vol. v1, pp. 273-275.

t+ Contributions to Mathematics, W. Shænks, London, 1853, pp. 86-87.

拉 Proceedings of the Royal Society, 1872-3, vol. xxI, p. 318; 1873-4, vol. IxII, p. 45. 
done in England, found the value of $\pi$ to 333 places* of decimals (330 correct); in 1854 he carried the approximation to 400 places †; and in 1855 carried it to 500 placest.

Of the series and formulae by which these approximations have been calculated, those used by Machin and Dase are perhaps the easiest to employ. Other series which converge rapidly are the following:

and

$$
\frac{\pi}{6}=\frac{1}{2}+\frac{1}{2} \cdot \frac{1}{3.2^{3}}+\frac{1.3}{2.4} \cdot \frac{1}{5.2^{3}}+\ldots
$$

$$
\frac{\pi}{4}=22 \tan ^{-1} \frac{1}{28}+2 \tan ^{-1} \frac{1}{443}-5 \tan ^{-1} \frac{1}{1393}-10 \tan ^{-1} \frac{1}{11018}
$$

the latter of these is due to Mr Escott§.

As to those writers who believe that they have squared the circle their number is legion and, in most cases, their ignorance profound, but their attempts are not worth discussing here. "Only prove to me that it is impossible," said one of them, "and I will set about it immediately"; and doubtless the statement that the problem is insoluble has attracted much attention to it.

Among the geometrical ways of approximating to the truth the following is one of the simplest. Inscribe in the given circle a square, and to three times the diameter of the circle add a fifth of a side of the square, the result will differ from the circumference of the circle by less than one-seventeenthousandth part of it.

An approximate value of $\pi$ has been obtained experimentally by the theory of probability. On a plane a number of equidistant parallel straight lines, distance apart $a$, are ruled; and a stick of length $l$, which is less than $a$, is dropped on the plane. The probability that it will fall so as to lie across one of the lines is $2 l / \pi a$. If the experiment is repeated many hundreds

- Grïnert's Archiv, vol. xxı, p. 119.

+ Ioid., vol. xxm, p. 476 : the approximation given in vol. xxII, p. 473 , is correct only to $\mathbf{3 3 0}$ places.

† Ibia., vol. xxv, p. 472; and Elbinger Anzeigen, No. 85.

$\S$ L'Intermédiaire des Mathematiciens, Paris, Dec. 1896, vol. II, p. 276. 
of times, the ratio of the number of favourable cases to the whole number of experiments will be very nearly equal to this fraction: hence the value of $\pi$ can be found. In 1855 Mr A. Smith* of Aberdeen made 3204 trials, and deduced $\pi=3.1553$. A pupil of Prof. De Morgan*, from 600 trials, deduced $\pi=3 \cdot 137$. In 1864 Captain Fox $\dagger$ made 1120 trials with some additional precautions, and obtained as the mean value $\pi=3 \cdot 1419$.

Other similar methods of approximating to the value of $\pi$ have been indicated. For instance, it is known that if two numbers are written down at random, the probability that they will be prime to each other is $6 / \pi^{2}$. Thus, in one caset where each of 50 students wrote down 5 pairs of numbers at random, 154 of the pairs were found to consist of numbers prime to each other. This gives $6 / \pi^{2}=154 / 250$, from which we get $\pi=3 \cdot 12$.

* A. De Morgan, Budget of Paradoxes, London, 1872, pp. 171, 172 [quoted from an article by De Morgan published in 1861].

$†$ Messenger of Mathematics, Cambridge, 1873, vol. II, pp. 113, 114.

$\ddagger$ Note on $\pi$ by $\mathbf{R}$. Chartres, Philosophical Magazine, London, series 6, vol. xxx1x, March, 1904, p. 315. 


\section{INDEX.}

Achilles and the Tortoise, 84.

Agrippa, Cornelius, 138.

Ahrens, W. 113, 138.

Airy, Sir Geo. 108.

Aix, Labyrinth at, 186.

Alcuin, 2, 71.

Algebraic Integers, 41.

Alkborough, Labyrinth at, 186.

Alphabet, Morse, 313.

Amicable Numbers, 38.

Ampère, A. M. 263.

ANaLUAGMATIC ArRANGeMENTs, 65-66.

Anaxagoras, 350.

Anderson, T. 316.

Andrews, W. S. 157.

Angular Motion, 86.

Anstice, R. R. 194.

Antipho, 297.

Apollonius, 339, 342, 351.

Arago, F. J. D. 276.

Archimedes, 54, 90, 346, 350, 352, 354.

Archytas on Delian Problem, 340.

Argyle, Earl of, 299.

ARIxHMETic, HIGHER, 36-43.

Arithmetical Fallacies, 28-31.

Arithmetical Prodigirs, chap. xirI.

- Puzzles, 4-33.

- Recreations, chaps. I. II. XI.' XII.

- Restorations, 248-253.

ARIThmometers, chap. xill.

Arya-Bhata on $\pi, 351$.

Asenby, Labyrinth at, 186.

Augustus, 315.

Ajrton, W. E. on Magic Mirrors, 108.

Bachet's Problèmes, 2-25, 34-36, 71, $238,240$.

Bacon, Francis, 315.

Bailey, J. E. 292.

Baker, H. F. 235.

Ball, W. W. R. 321, 338 .

Barbette, E. 157.

Baudhayana on $\pi, 351$.

Bazeries, 311.

Beaufort Cipher, 308.

Begs and their Cells, chap. viII.

Bees' Cells, Shapes of, 161-162.

Benham, C. ‥ 108.

Bennett, G. T. 118.
Bergholt, E. G. B. 33.

Bernoulli, John, 29.

Berri, Duchesse de, 310.

Bertrand, J. L. F. 28.

Bertrand, I. (of Geneva), 124.

Berwick, W. E. H. 250.

Besant on Hauksbee's Law, 101.

Bewley, E. D. 33.

Bhaskara on $\pi, 352$.

Bickmore, C. E. 357.

Bidder, G. P. 271-275, 282-291.

Bidder Family, The, 259, 275.

Biering, C. H. 338.

Bilguer, von, on Chess Pieces, 112.

Binary Powmis, Fermat on, 39-40.

Binet, A. 279.

Birds, Flight of, 106-107.

Brshop's Re-entrant Pate, 134.

Boat-racing with a Rope, 101.

Bordered Magic Squares, 144-146.

Boscovich, R. 165.

Boughton Green, Labyrinth at, 186.

Bouniakowski, V. on Shuftling, 235.

Bourget, M. J. on Shuffling, 235.

Bourlet, C. E. E. 22, 23.

Bouton, C. L. 21.

Brahmagupta on $\pi, 352$.

Bray, A. on Kirkman's Prob., 194, 203.

Breton on Mosaics, 185.

Bridge, Problem, 222.

Bromton, 184.

Brougham, Lord, 165.

Brouncker, Viscount, on $\pi, 355$.

Bryan, G. H. on Bird Flight, 106, 107.

Bryant, S. 167-169.

Bryso, 350.

Busschop, P. 258.

Button-Hole, String Trick, 335-336.

Buxton, Jedediah, 265-268.

Caesar, Julius, 315.

Calculating Prodiaies, chap. xiri.

Calcolation, Mental, chap. xill.

Calendar Problems, 253.

Callet, J. F. on $\pi, 357$.

Cantor, M. on $\pi, 349$.

Cardan, G. 2, 229, 232, 292.

Cards, Problems with, 16-18, 32, 33, $71,235-246$.

Caroline Catch, String Trick, 330-333. 
Cartwright, W. 316.

Caterpillar, String Figure, 330-331.

Cat's Cradles, chap. Xv.

Cauchy, A. L. 61, 260.

Cayley, A. 55, 59, 188, 245, 355.

Cells of a Chess-board, 122.

Centrifugal Force, 89.

Ceulen, van, on $\pi, 354,355$.

Charcot, J. M. 279.

Charles I, 316, 317.

Chartres, Labyrinth at, 186.

Chartres, R. 28, 51, 359.

Chasles on Trisection of Angle, 346.

Chess-board, Games on, 74-80, ohap.vi.

- Problems, 74-80, chap. vi.

- Knights' move on, 111.

- Notation of, 109.

- Recreations, chap. vi.

Chess, Maximum Pleces Prob, 119.

- Minimur Precter Prob., 119.

- Number of Initial Moves, 110.

- Pieces, Valde of, 110-113.

Chifu-Chimolpo Puzzlw, 70, 82.

Chilcombe, Labyrinth at, 186.

Chinese on $\pi, 352$.

Chingase Rings, 229-234.

Chippewa Release, String Trick, 333.

Ciccolini, T. on Chess, 129.

Ciphers, chap. xiv.

- Definition of, 293

- Four types of, 300-310.

Circle, Quadratide of, 346-359.

Cissoid, the, $340,342,348$.

Clairaut on Trisection of Angle, 345 .

Claus, 228.

Clausen on $\pi, 357$.

Clerk Maxwell, J. 5, 59, 108.

Clifford, W. K. 87.

Clocks, 96

Cnossus, Coins of, 184, 185.

Coat and Waisteoat Trick, 336.

Coccoz, M. 46, 157.

Code-Book Ciphers, 318.

Code, Morse, 313.

Colburn, Z. 269-271, 272.

Cole, R. A. L. 262.

Colebrooke, H. T. 299.

Collini on Chess, 128.

Collins, Letter from J. Gregory, 303.

Colodr-cube Problem, 67-69.

Colodring Maps, 54-59.

Columbus's Egg Puzzle, 93.

Comberton, Labyrinth at, 186.

Compass Problems, 262-263.

Conchoid, the, 340, 344, 348.

Cones moving uphill, 93.

Conrad's Tables, 302 .

Contour-lines, 59-60.

Counters, Games with, 62-64, 74-80.

Cox, James, on Clocks, 96.
Cradle, String Figure, 331.

Cretan Labyrinth, 182, 184-185.

Cricket-baul, Spin on, 105.

Cross-fours, 67.

Cryptographs, chap. xiv.

- Definition of, 293.

- Three types of, 294-300.

Cryptugraphy, chap. xiv.

Cobe, Duplication of, 338-344.

Cubes, Coloured, 67-69.

- Skeleton, 32.

Cudworth, W. on Sharp, 356.

Cumulative Vote, 33.

Cunningham, A. J. C. 40.

Curiosa Physica, 107-108.

Corl on a Cricket-bali, 105.

Cusa on $\pi, 353$.

Cut on a Tennis-ball, 103-105.

Cutting Cards, Problems on, 17.

D'Alembert, J. 28, 30.

Darboux, G. 279, 282.

Dase, J. M. Z. 276-278, 291, 357, 358.

Davis, E. P. on Kirkman's Prob., 218.

De Berri, Duchesse, 311.

Decimation, 24-27.

De Coatpont, 258.

De Fonteney on Ferry Problem, 72.

De Fouquières, 63.

De Haan, B. on $\pi, 349,354$.

De Lagny on $\pi, 356$.

De la Hire, P. 147, 150.

De la Loubère, S. 139, 151.

Delannoy, 72.

Delastelle, F. 292.

De Lavernede, J. E. T. 129.

Delens, P. 248, 249.

Delian Problem, 338-344.

De Moivre, A. 122, 123.

De Montmort, 1, 123.

De Morgan, A. 55, 84, 346, 348, 349, 359.

Denary Scale of Notation, 10-11.

De Parville on Tower of Hanoi, 229.

De Polignac on Knight's Move, 133.

De Rohan, 318.

Derrington, on Queens Problem, 118.

De St Laurent, T. 235.

Descartes, 343, 348, 356.

Diabolic Magic Squares, 150-156.

Diamandi, 279.

Dickson, L. E. 38, 41, 42, 244.

Diego Palomino, 23.

Digby, Lord, 316.

Digit Questions, 247.

Digital Process, 285.

Diaits, Missing, 248-253.

Diocles on Delian Problem, 342.

Diodorus on Lake Moeris, 184.

Diroks, H. 94, 318. 
Dirichlet, Lejeune, 42.

Dissection Problems, 54, 256-260.

Dissection, Proofs by, 52-54, 256-260.

Dodrcaredron GaMe, 189-192.

Dodgson, C. L. on Parallels, 45.

Dominoes, 22-23, 168-169.

Dominoes, ArRanemments of, 178-181.

Doubly-Magie Squares, 156-157.

Drayton, 184.

Dudeney, H. E. 26, 33, 47, 72, 119, $160,194,203,259$.

Doplication OF CoBe, 338-344.

Dürer, A. 138.

Dynamical G

Earnshaw, S. 108.

Eckenstein, O. on Kirkman's Problem, 193, 199, 203, 209, 217, 220, 222.

Eight Quenes Probiem, 113-118.

Eisenlohr, A. on Ahmes, 350 .

Eisengtein, F. G. 40.

Ellis, R. Leslie, 166.

Elusive Loop, String Trick, 332.

Encke, J. F. 277.

Eneström, G. on $\pi, 349$.

Epicurus on Gravitation, 171.

Equilibrium, Puzzles on, 90-93.

Eratosthenes, 340 .

Escott, E. B. 160, 305.

Etten, van, 11.

Euclid, 38, 44-45, 297.

Euc. I. 32, 52.

Euc. I. 47, 52, 256, 257.

Euler, L. 38-41, 61, 122-127,' 150, 158, $335,356$.

Eulerian Squares, 159.

Euler's Officers Problem, 158.

Euleh's Unicorgal Prob., 179-182.

Euzet, M. 258.

Exploration Problems, 23.

Fabre, J. H. 164.

Fairfax, 316.

Faldacies, Arithmetical, 28-31

- GromethicaL, 44-52.

- MECHANICAL, 84-87, 93-98.

Fermat, P. 36-43.

Fermat on Binary Powers, 39-40.

Fermat's last theorem, 40-43.

Ferry-bont Problems, 71-73.

Fifteen Girls Problem, chap. x.

Fifteen Pozzle, 224-228.

Firth, W. 143.

Fitzpatrick, J. 75.

Five Diso Problem, 253-255.

Fluid Motion, 101-107.

Fly, String Figure, 332-333.

Fly-on-the-Nose, String Trick, 334-335.

Fonteney on Ferry Problem, 72.

Force, Definition of, 87.
Fortunatianus, 54.

Fouquières, Becq de, on Games, 63.

Four-Colour Map Theorem, 54-59.

Four " 4's" Problem, 14.

Four " 4's," Analogous Problems, 14.

Four Digits Problem, 13.

Fourrey, E. 253.

Fox, Captain on $\pi, 359$.

Frankenstein, G. 148.

Frénicle (de Bessy), B. 144.

Frost, A. H. 150, 194.

Fuller, T. 268.

Galton, F. 28.

Games, Dynamica1, 69-80.

- Statical, 62-69.

-- with Counters, 74-80.

Gauss, C. F. 43, 269, 277.

Geodesic Problems, 73-74.

Geography, Physicax, 59-61.

Geometrical Fallaciss, 44-52.

- Problems, Three Classical, chap. xvI.

- ReCREATIONs, chaps. III. IV. XI. XII.

Gerbert, 298.

Grigonnis's Problem, 240-244.

Germain, S. 42.

Gerwien, P. 258.

Gill, T. H. on Kirkman's Prob., 194.

Glaisher, J. W. L. 114, 163, 349.

Glamorgan, Earl of, 317.

Goldbach's Theorem, 39.

Golf-balls, Fuight of, 105.

Great Northern Puzzle, 69, 82.

Greenwich, Labyrinth at, 186.

Gregory, Jas. 347, 355.

Gregory of St Vincent, 343.

Gregory's Series, 356.

Grienberger on $\pi, 356$.

Grille, The, 297.

Gronfeld's Method in Ciphers, 306.

Gros, L. on Chinese Rings, 232, 234.

Goarini's Problems, 135-136.

Guitel, E. 258.

Gun, Report of, 108.

Günther, S. 113, 139.

Guthrie on Colouring Maps, 55.

Haan, B. de on $\pi, 349,354$.

Haddon, K. 332.

Halley on $\pi, 356$.

Halter, String Trick, 333.

Hamilton, Sir Wm. 189-192.

Hamiltonian Game, 189-192.

Hampton Court, Maze at, 182, 186.

Handcuff, String Figure, 335 .

HANOI, TOWER OF, 228-229.

Harison, G. D. L. 194, 218.

Harrison, C. H. 320.

Barzer, P. on $\pi, 352$. 
Hatksbets LAW, 101-106.

Hayward, Sir J. 302.

Heawood, P. J. on Maps, 56.

Hegesippus on Decimation, 24.

Helmboltz, H. L. F. von, 97.

Henry, Ch. on Euler's Problem, 170.

Hermann, A. 65.

Hermary, 192.

Herodotus on Lake Moeris, 184.

Hero of Alexandria on $\pi, 340,351$.

Herschel, Sir John, 272.

Herschel, Sir William, 273.

Hexagon Problem, 256.

Hiero of Syracuse, 90.

Higher Arithuetic, 36-43.

Hills and Dates, 59-60.

Hippias, 350.

Hippocrates of Chios, 340, 350.

Hoffmann, L. 332.

Holtz, E. 258.

Houdin, J. E. R. 278.

Huber, F. 164.

Hudson, G. T. 244.

Hudson, W. H. H. 236.

Hutton, C. 3, 356.

Huygens, C. $342,346,355$.

Hyper-magio SQdaris, 150-158.

Icostan Game, 189-192.

Inaudi, J. 279-282.

Inertia, 88, 89.

Integers, Algebraic, 41.

Internal Structure, Test of, 93.

Inwards on the Cretan Maze, 181.

Jqenisch, C. F. de, 120, 122, 128, 132.

James II of England, 299.

Japanese Magio Mirrors, 108.

Jayne, C. F. on String Figures, 332.

Jephson, T. 272.

Johnson, W. on Fifteen Puzzle, 224.

Jones, W. on $\pi, 349,356$.

Josephus Problem, 23-27.

Julian's Bowers, 186.

Julius Caesar, 312.

Kempe, A. B. on Colonring Maps, 56.

King's Re-entrant Path, 133.

Kirkman, T. P. 193.

Kirimay's Problem, chap. $x$.

Klein, F. C. 284.

Klüber, J. L. 292.

Kntaht, Re-entrant Path, 122-132.

Kniahts of the RodNd TABLe, 33.

Knyghton, 184.

Koenig, S. 162-164.

Königsberg Problem, 170-183.

Kummer, E. E. 42.

Labyrinths, 182-187.
Lacroix, P. L. 346.

Lagny on $\pi, 356$.

Lagrange's Theorem, 39.

La Hire, P. 147, 150.

Laisant, C. A. 12.

La Loubère, S. 139, 151.

Lambert, J. H. on $\pi, 346,347$.

Lamé, G. 42.

Langley, S. P. on Bird Flight, 106.

Laquiere on Knight's Path, 131.

Latin Squares, 136.

Latruaumont, 318.

Lavernedde, J. E. T. de, 129.

Lawrence, F. W. 37.

Leake, 11, 14, 18, 22.

Lebesgue on Fermat's Theorem, 42.

Legendre, A. M. 42, 124, 132, 293, 294.

Legros, L. A. 194, 195.

Lejeune Dirichlet on Fermat, 42.

Leonardo of Pisa on $\pi, 353$.

Leslie, J. 340, 344.

Leurechon, J. 2, 11.

Lhulier, S. 165.

Lightning, String Figure, 326-327.

Linde, A. van der, 122.

Lindemann on $\pi, 347$.

Lines of Slope, 60 .

Listing, J. B. 81, 172, 379.

Liveing on the Spectrum Top, 108.

Lizard Twist, String Trick, 332-333.

Loudon and Wise, 186.

Loubère, S. de la, 139, 151.

Loyd, S. 19.

Lucas, E. 67, 72, 77, 79, 80, 170, 178, $183,218,228,232$.

Lucas di Burgo, 2.

Lucos, Labyrinth at, 186.

Macaulay, W. H. 259-260.

McClintock, E. 145.

Machin's Series for $\pi, 356,357,358$.

Maclaurin, C. 164, 472.

MacMahon, P. A. 34, 35-36, 67, 136. 255.

Maeterlinck, M. 164.

Magic Bottles, 90, 91.

Magic Mirrors, 108.

Magro Pencils, 163-165.

Magio Product Squares, 147.

Magio Squares, chap. vir.

Magic Square Puzzles, 158, 160.

Magio Stars, 147-148.

Magnus on Hauksbee's Law, 103.

Mangiamele, V. 276.

MAP ColodR Theorey, 54-59.

Maraldi, G. F. 163.

Marie Antoinette, 318.

Marsden, E. on Kirkman's Prob., 124.

Mascheroni, L. 262, 263. 
Maxim, H. on Bird Flight, 106.

Maxwell, J. Clerk, 5, 59, 108.

Maxwell's Demon, 108.

Mazes, 182-187.

Mechanical Recreations, chap. v.

Medieval Problems, 18-25.

Menaechmus, 343.

Ménage Problem, 34.

Mental AaIthMetic, chap. xir.

Mersenne on Primes, 37.

Mersenna's Nombers, 37.

Mesolabum, 340.

Metius, A. on $\pi, 353$.

Méziriac, see Bachet.

Mikami, Y. 145.

Miller, G. A. 222.

Minding on Knight's Path, 132

Minos, 182, 339.

Minotaur, 184.

Mirrors, Magic, 108.

Miscellaneods Problems, 224.

Mrssing Digits, 248-253.

Mitchell, F. D. 264.

Möbius, A. F. 55.

Models, 97-98.

Mohammed's Sign-Manual, 176.

Moivre, A. de, 122, 123.

Mondeux, H. 276, 281.

Money, Question on, 9-10.

Monge on Shuffling Cards, 235-237.

Montmort, de, 1, 123.

Montucla, J. F. 3, 90, 91, 123, 147, $258,346,347,355$.

Moon, R. 132.

Moore, E. H. 222.

Morcom, R. K. 21.

Mordell, L. J. 41.

Morehead, J. C. 40.

Morgan, A. de, see De Morgan.

Morse Code, 315-316.

Mosaic Pavements, 64, 185.

Moschopulus, 138.

Mosquito, String Figure, 322-323.

Motion in Fluids, 101-107.

Motion, Laws of, 83, 87-93.

- Paradoxes on, 84-87.

- Perpetual, 93-96.

Modsetrap, Game of, 245-246.

Müller (Regiomontanus), 300.

Müller, G. E. 264.

Mydorge, 2.

Nasik Magio Squares, 150-156.

Nauck, F. 113.

Navahoing Loops, String Figure, 326.

Net, String Figure, 327-328.

Neville, E. H. 254.

Newton, Isaac, 94, 103, 343, 345, 347, 348.

Newtonian Laws of Motion, 83-93.
Niceron, J. F. 292.

Nicomedes, 340.

Nim, 21.

Notation, Denary Scale of, 10-11.

Noughts and Crosses, 62.

Nunibers, Perfect, 38.

- Puzzles with, 4-27.

- Theory of, 36-43.

Openings, A, B, String Figure, 326.

Oppert on $\pi, 297$.

Oram on Eight Queens, 117.

Oughtred's Recreations, 11, 14, 18, 22, $91,92$.

Ovid, 184.

Ozanam, A. F. on Labyrinths, 185.

Ozanam's Récréations, $2,3,11,18$, $25,53,71,90,91,92,93,96,98$, $123,138,139,149,166,229$.

$\pi, 293-306$; see Table of Contents.

Pacioli di Burgo, 2.

Pairs-of-Cards Trick, 238-240.

Palomino, 23.

Pandiagonal Magic Squares, 150-156.

Pappus, 340, 344, 345.

Paradromio Rings, 80-81.

Parmentier on Knight's Path, 122.

Parry on Sound, 108.

Parville, de, 229.

Pawns, Games with, 74-80.

Peirce, B. on Kirkman's Problem, 104.

Peirce's Problem of $n^{2}$ Girls, 219.

Pepys, S. 317.

Perfect Magic Squares, 150-160.

Perfect Numbers, 38.

Permutation Problems, 32.

Prratedal Motion, 93-96.

Perrin, 12.

Perry, J. on Magic Mirrors, 108.

Petersen, A. C. 277.

Petersen, J. on Maps, 57.

Philo, 287.

Philoponus on Delian Problem, 338.

Physical Geography, 59-61.

Prie Problems, 240-245.

Pirie, G. on $\pi, 355$.

Pittenger, W. 89.

Planck, C. 159.

Plato on Delian Problem, 338, 339.

Playfair Cipher, 308.

Pliny, 184.

Poe, E. A. 292, 302.

Poitiers, Labyrinth at, 186.

Pole, W. 290.

Polignac on Knight's Path, 139.

Porta, G. 292.

Portier, B. on Magic Squaree, 157.

Pratt on Knight's Path, 128.

Pretender, The Young, 299. 
Primes, 37.

Probabilities and $\pi, 358$.

Probsbilities, Fallacies in, 30-32, 52.

Ptolemy, 298.

Purbach on $\pi, 353$.

Puzzies, Arithmetical, 4-36.

- Geometrical, 62-81.

- Mechanical, 84-93.

Pythagorean Symbol, 176.

Quadrature of Crrcle, 346, 359.

Quien, Paths ox Chess-board, 133, $134,135$.

Quenns Problem, Eraht, 113-118.

Queens, Problems with, 113-118.

Racquet-ball, Cut on, 103-105.

Railway Puzzles (Shunting), 69-71.

Ramification, 188.

Ravenna, Labyrinth at, 186.

Rayleigh, Lord, 103, 105, 106.

Réaumur, F. A. F. 164.

Re-entrant Chess Paths, 122-134.

Regiomontanus on $\pi, 353$.

Reimer, N. T. 338.

Reiss, M. 80, 181.

Relative Motion, 87.

Renton, W. 52.

Restorations of Digits, 248-253.

Rhind Papyrus, 350.

Rich, J. 317.

Richard, J. 87.

Richelieu, 298.

Richter on $\pi, 357$.

Rilly, A. 157.

Robert-Houdin, J. E. 278.

Rockliff Marshes, Labyrinth at, 186.

Rodet, L. on Arya-Bhata, 351.

Roget, P. M. 122, 127-132.

Romanus on $\pi, 353$.

Rome, Labyrinth at, 186.

Roor, Re-entrant Pate, 133-134.

Rosamund's Bower, 184.

Rosen, F. on Arab values of $\pi, 352$.

Rodnd Tabue, Knights of, 33.

Route Method in Ciphers, 296.

Routes on Chess-board, 122-135.

Row, Counters in a, 62-64, 74-78.

Rückle, C. 279, 281, 282.

Rudio, $\mathrm{F}$, on $\pi, 346$.

Russell, B. A. W. 85 .

Ratherford on $\pi, 357$.

Safford, T. H. 278, 279.

Baffron Walden, Labyrinth at, 186.

SaIliva, Theory of, 98-101.

St Cyr Method in Ciphers, 307.

Bt Laurent, T. de, on Cards, 235.

St Omer, Labyrinth at, 186.

St Vincent, Gregory of, 343.
SaImon Net, String Figure, 327-328.

Sauveur, J. 150.

Scale of Notation, Denary, 10-11.

- Puzzles dependent on, 11-14.

Sozool-girls, Firtere, 193-223.

Schooling, J. H. 10.

Schub, H. 249.

Schubert, H. on $\pi, 346$.

Schumacher, H. C. 277.

Scripture, E. W. 264.

Scytale, The, 300.

Secret Commonications, chap. zim.

Selander, K. E. I. on $\pi, 349$.

Seventy-seven Puzzle, 54.

Shanks, W. on $\pi, 357$.

Sharp, A. on $\pi, 356$.

Bhelton, T. 316.

Sherwin's Tables, 356.

Shoffling Cards, 235-237.

Bhonting Problems, 69-71, 82.

Sixteen Counter Problem, 64, 82.

Sixty-five Puzzle, 52-53.

Skeleton Cubes, 32.

Smitb, A. on $\pi, 359$.

Smith, D. E. 145.

Smith, H. J. S. 42.

Snell on $\pi, 354,355$.

Solitaire, 80.

Sound, Problem in, 107-108.

Southwark, Labyrinth at, 186.

Sovereign, Change for, 32.

Spectrum Top, 108.

Spin on a Cricket-ball, 105.

Sporus on Delian Problem, 342.

Sedaring tere Circle, 346-359.

Stability of Equilibrium, 90-93.

Statical Games, 62-69.

Steen on the Mousetrap, 246.

Storey on the Fifteen Puzzle, 224.

Strabo on Lake Moeris, 184.

Strachey, R. 140, 148.

Strasznicky, 277, 278.

String Figures, chap. xp.

- Tricrs, 332-336.

Sturm, A. 338.

Suetonius, 315.

Super-Dominoes, 255-256.

Suter, H. 54.

Svastika, 185.

Swift, J. 84.

Swinden, B. A. 27.

Sylvester, J. J. 63, 65, 222.

Symmetrical Magic Squares, 156.

Tait, P. G. 25, 56, 57, 58, 75, 172, 176. Tangrams, 69.

Tanner, L. on Shuffling Cards, 235.

Tarry, G. 72, 157, 158, 177, 178.

Tartaglia, 2, 18, 24, 34, 71 .

Taylor, B. 123. 
Taylor, Ch. 345.

Taylor, H. M. 110, 259.

Tennis-ball, Cut on, 103-105.

Tent Flap, String Figure, 325-326.

Tesselation, 64-67, 255-256.

Texeira, F. G. 337.

Theory of Nombers, 36-43.

Thibant, G. on Baudhayana, 351.

Thompson, T. P. on Parallels, 307.

Thomson, J. J. 105.

Threading Needle, String Trick, 333334.

Three-ny-A-ROW, 62-64.

Three-Puje Problem, 240-245.

Three-Things Problem, 12-23.

Tom Tit, 332.

Towen of HANö̈, 228-229.

Trastevere, Labyrinth at, 186.

Trebly-Magio Sqdares, 158.

Trees, Geometrical, 188.

Treize, Game of, 245-246.

Trémaux on Mazes, 183.

Tricks With numbers, 3-34.

Trisection of Angle, 344-346.

Tritheim, J. 292.

Trollope, E. on Mazes, 184.

Troy-towns, 186.

Turtoin, W. H. 49, 121.

Two-Digit Process, 285.

Unicorsat Problems, chap. Ix.

Van Ceulen on $\pi, 354,355$.

Vandermonde, 80, 122, 127.

Van Etten, H. 11.

Varignon, P. on Parallels, 322.

Vase Problem, 18.

Vega on $\pi, 304$.

Victorinus, 54.
Vieta, F. 343, 355.

Violle, B., Magic Squares, 139.

Virgil, 184.

Volpicelli, P. on Knight's Path, 122.

Von Bilguer on Chess Pieces, 112.

Von Helmholtz, H. F. L. 97.

Voting, Question on, 33.

Waistcoat Trick, 336.

Walecki on Kirkman's Prob., 218.

Walker, G. T. 28.

Wallis, J. 229, 232, 265, 355.

Wantzell, P. L. 284.

Warnsdorff, Knight's Path, 128.

Watch Problem, 14-15.

Watches, 96.

Watersheds and Watercourses, 60-61.

Waves, Superposition of, 108.

Weights Problem, The, 34-36.

Western, A. E. on Bivary Powers, 40.

Whately, R. 269.

Whertstone, C. 311, 316, 317.

Whist, Number of Hands at, 33.

Wiedemann, A. on Lake Moeris, 184.

Wieferich, A. 42

Wilkins, J. 292, 300, 307, 309.

William III of England, 186.

Willis on Hauksbee's Law, 101.

WiNDOW READER, 260-262.

Wing, Labyrinth at, 186.

Work, 89-93.

Wostrowitz, E. B. von, 292.

Yam Thief, String Figure, 328-330.

Zach, F. X, von on $\pi, 357$.

Zamebone, U. 279.

Zeno on Motion, 84-85. 


\section{WORKS BY W. W. ROUSE BALL.}

A Short History of Mathematics.

Stereotyped Edition. Pp. xxiv + 522. Price 12s. 6d. net. Macmillan \& Co., Ltd.

A Primer of the History of Mathematics.

Fourth Edition. Pp. iv + 149. Price 2s. 6d. net. Macmillan \& Co., Ltd.

Mathematical Recreations and Essays.

Tenth Edition. Pp. xiii +366 . Price ros. 6d. net. Macmillan \& Co., Ltd.

String Figures, An Amusement for Everybody.

Second Edition. Pp. 69. Price 2s. 6d, net. W. Heffer \& Sons, Ltd.

A History of the Study of Mathematics at Cambridge. Pp. xvi +264 . Out of print. Cambridge University Press.

An Essay of the Genesis, Contents, and History of Newton's Principia.

Pp. $x+175$. Out of print. Macmillan \& Co., Ltd.

Cambridge Notes (originally published under the title Cambridge Papers).

Pp. viii + 332. Price 21s. net. W. Heffer \& Sons, Ltd. 






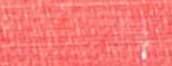

